\title{
Optimal Design of Composite Fuselage Frames for Crashworthiness
}

\author{
by \\ Marshall Benjamin Woodson
}

Dissertation submitted to the faculty of the

Virginia Polytechnic Institute and State University

in partial fulfillment of the requirements for the degree of

DOCTOR OF PHILOSOPHY

in

Aerospace Engineering
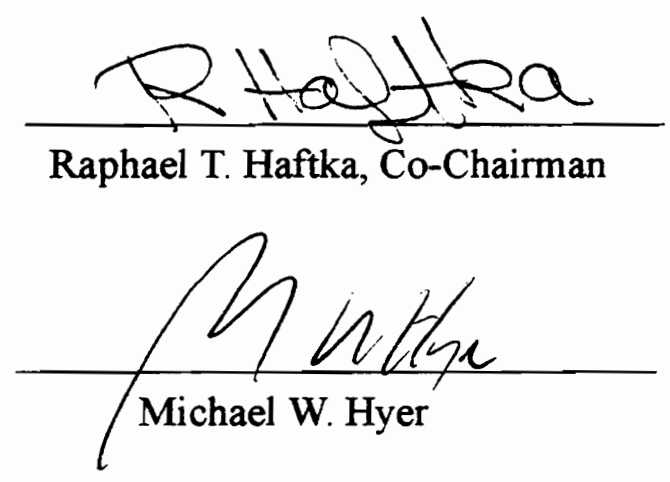
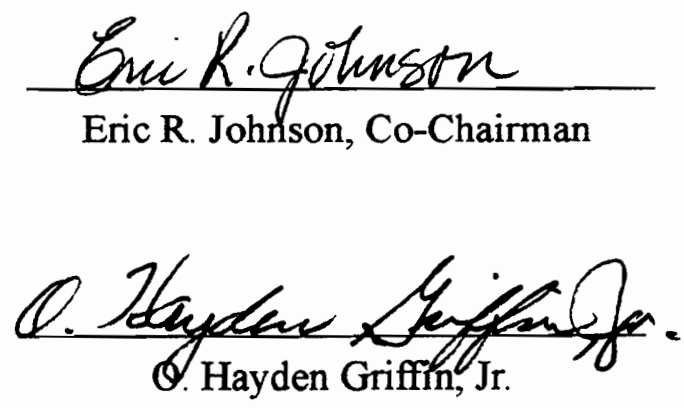

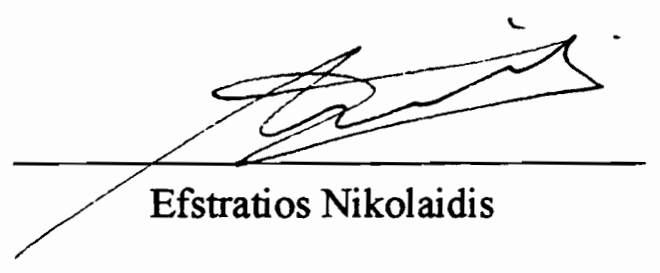

December, 1994

Blacksburg, Virginia 


\title{
Optimal Design of Composite Fuselage Frames for Crashworthiness
}

\author{
by \\ Marshall Benjamin Woodson \\ Raphael T. Haftka and Eric R. Johnson, Co-Chairmen \\ Aerospace Engineering
}

(ABSTRACT)

This study looks at the feasibility of using structural optimization techniques to address the problem of designing composite fuselage frames for crashworthiness. A key feature of any optimization strategy for increasing structural crashworthiness is a progressive failure analysis. Currently, the most widely used analysis methods for progressive failure of composite structures are considered too expensive computationally for practical optimization in todays computing environment. Developing an efficient analysis method for progressive failure of composite frames is a first step in the optimization for crashworthiness. In the current work, a progressive failure analysis for thin-walled open cross-section curved composite frames is developed using a Vlasov type beam theory. A curved thin-walled composite beam theory is developed and a finite element implementation of the beam theory is used for progressive failure analysis. The accuracy and limitations of this analysis method are discussed. A model for progressive failure of the composite fuselage frame is developed from an extension of the laminate progressive failure analysis of Tsai-Wu. Comparisons based on a limited amount of available experimental data are encouraging. The first major failure event is captured by the theory, and the prediction of total energy absorbed follows the trend of the experimental data. It is believed that this accuracy is sufficient for preliminary design and optimization for crashworthiness. This progressive failure analysis is then incorporated into a frame optimization for crashworthiness based on the genetic algorithm method. The optimization methodology is demonstrated analytically to obtain frame designs with substantially increased crashworthiness. Laminate stacking sequence and cross-section shape are design variables for optimization. 


\section{Acknowledgments}

This work was funded through the NASA-Virginia Tech Composites Program.

I would particularly like to thank my advisors and co-chairmen Dr. Raphael T. Haftka, and Dr. Eric R. Johnson for their valuable help and assistance during the course of this work. I would also like to thank my grant monitors Mr. Huey Carden, and Dr. Richard Boitnott for their support of this project, and for the opportunity to spend time at the NASA Langley Research Center. I would like to thank Dr. Michael W. Hyer for agreeing to serve on my committee, and for my acceptance into the Virginia Tech Composites Program. The support provided by NASA and the Virginia Tech Composites program is greatly appreciated. Finally, I would like to thank Dr. O. Hayden Griffin Jr., and Dr. Efstratios Nikolaidis for agreeing to serve on my committee. 


\section{TABLE OF CONTENTS}

ACKNOWLEDGMENTS

TABLE OF CONTENTS

LIST OF FIGURES

VII

LIST OF TABLES

XII

1 STRUCTURAL OPTIMIZATION FOR CRASHWORTHINESS

1

1. 1 General principles of crashworthy design

1

1. 2 Structural requirements for crashworthiness

2

1. 3 Mechanisms for absorbing vertical crash impact energy

7

1. 4 Special considerations for composite structures

8

1. 5 Composite frame design for crashworthiness

9

1. 6 Optimization and analysis for crashworthiness 11

1. 7 Scope and objective 


\section{ANALYSIS OF THIN-WALLED LAMINATED COMPOSITE CURVED} BEAMS

2. 1 Theory of thin-walled curved beams 17

2. 2 Coordinate systems 21

2. 3 Coordinate relations 24

2. 4 Kinematics of deformation 28

2. 5 Strain-displacement $\quad 38$

2. 6 Strain energy $\quad 48$

2. 7 Hooke's law $\quad 50$

3 CALCULATION OF CURVED BEAM SECTION PROPERTIES 60

3. 1 Introduction $\quad 60$

3. 2 Evaluation of section contour integrals 60

3. 3 Evaluation of the contour warping function 68

$\begin{array}{lll}3.4 \text { Program verification } & 70\end{array}$

4 FINITE ELEMENT DEVELOPMENT

4. 1 Introduction $\quad 72$

4. 2 Element formulation 72

4. 3 Displacement approximation $\quad 78$

4. 4 Static condensation $\quad 82$

4. 5 Element transformation 83

4. 6 Calculation of direction cosines 88

5 ANALYSIS VERIFICATION 92

5. 1 Introduction 92

5. 2 Finite element evaluation 92 
5. 3 Comparison to branched shell analysis 96

5.4.1 Influence of in-plane distortion of the cross section 103

5.4.2 Influence of laminate stacking sequence and loading 105

6 PROGRESSIVE FAILURE ANALYSIS

6. 1 Introduction 111

6. 2 Progressive failure analysis 113

6. 3 Material failure 117

6. 4 Evaluation and comparison to test data 125

6. 5 Sensitivity to mesh discretization 130

6. 6 Sensitivity of energy absorbed to failure envelope 132

7 OPTIMAL FRAME DESIGN FOR CRASHWORTHINESS

7. 1 Problem formulation 134

7. 2 Genetic algorithms 139

7. 3 Fundamentals of genetic algorithms 140

7. 4 Algorithm implementation 145

7.4.1 Genetic coding for laminate design 145

7.4.2 Establishing population size and probability of mutation 147

7.4.3 Crossover rate and convergence. 150

7.4.4 Fitness calculation 151

7.4.5 Genetic optimization of energy absorption 153

7. 5 Optimization results and discussion 154

7.5.1 Laminate optimization 154

7.5.2 Cross-sectional geometry optimization $\quad 159$

7.5.3 Separation of failure events 164 
8 SUMMARY AND CONCLUDING REMARKS

8. 1 Summary

170

8. 2 Concluding remarks

174

8. 3 Future work

175

9 REFERENCES 


\section{LIST OF FIGURES}

$\begin{array}{lll}\text { Figure } 1.1 & \text { Tailoring impact accelerations for survivability } & 3\end{array}$

Figure 1.2 Optimal failure response 5

$\begin{array}{lll}\text { Figure 1.3 Typical crash scenario } & 6\end{array}$

Figure 1.4 Major sources of vertical impact energy absorption $\quad 7$

$\begin{array}{lll}\text { Figure 1.5 Aluminum fuselage crushing behavior and energy absorption } & 8\end{array}$

Figure 1.6 Composite fuselage crushing behavior and energy absorption 9

$\begin{array}{lll}\text { Figure } 1.7 \quad \text { Analysis structure. } & 10\end{array}$

$\begin{array}{lll}\text { Figure 2.1 Cross section terminology } & 18\end{array}$

Figure 2.2 Example of the bimoment resultant 20

Figure 2.3 Cylindrical and cross section coordinates. 23

Figure 2.4 Contour coordinates and sign convention. 24

$\begin{array}{lll}\text { Figure 2.5 Relationship between contour coordinates } & 26\end{array}$

Figure 2.6 Rotation of position vector $\mathrm{r}$ to through angle $\beta$ about the axis of rotation .

Figure 2.7 Displacements of the beam cross section 34 
Figure 2.8 Bar displacements and rotations referenced to point $\mathrm{R}$

Figure 2.9 Contour warping function

Figure 2.10 Positive bar resultants

Figure 3.1 Straight branch contour element

Figure 3.2 Location of principal contour origin.

Figure 4.1 Three node curved beam element

Figure 4.2 Cartesian degrees of freedom

Figure 4.3 Element description

Figure 4.4 Element end rotation

Figure 5.1 Circular arch under a central load

Figure 5.2 Straight beam test geometry

Figure 5.3 Beam cross sections for EAL comparisons

Figure 5.4 Curved beam test geometry

Figure 5.5 Blade section compliance ratios $(\mathrm{b} / \mathrm{t}=17.85)$

Figure 5.6 Angle section compliance ratios $(b / t=17.85)$

Figure 5.7 I - section compliance ratios $(b / t=17.85)$

Figure 5.8 I - section compliance ratios $(\mathrm{b} / \mathrm{t}=8.92)$

Figure 5.9 Channel section compliance ratios $(b / t=17.85)$

Figure 5.10 Channel section compliance ratios $(b / t=11.9)$ 102

Figure 5.11 Cross section distortion

Figure 5.12 Torsional related distortion for curved beams.

Figure 6.1 Computational diagram of progressive failure analysis 
Figure 6.2 Progressive failure sequence

Figure 6.3 Flow chart for lamina degradation

Figure 6.4 Curved test frame geometry and cross section

Figure 6.5 Comparison of progressive failure to experimental results for frame $1 \mathrm{~b} 128$

Figure 6.6 Extended comparison of progressive failure to experimental results for frame $1 \mathrm{~b}$

Figure 6.7 Comparison of energy absorption.

Figure 6.8 Effect of mesh discretization

Figure 6.9 Progressive failure using 16 beam elements

Figure 6.10 Progressive failure using 30 beam elements

Figure 6.11 Progressive failure using 50 beam elements

Figure 7.1 Optimal design problem for maximizing frame crashworthiness

Figure 7.2 Nonlinearity of energy absorption .vs. ply angle (angle ply laminate)

Figure 7.3 Discontinuity of Energy absorption.

Figure 7.4 Nonlinearity of energy absorbed .vs. section shape

Figure 7.5 Example coding sequence

Figure 7.6 Reproduction

Figure 7.7 Crossover

Figure 7.8 Mutation

Figure 7.9 Probability of mutation .vs. reliability

Figure 7.10 Probability of mutation .vs. normalized cost.

Figure 7.11 Population size .vs.reliability 
Figure 7.12 Population size .vs.cost

Figure 7.13 Fitness scaling

Figure 7.14 Flow diagram of optimal design using Genetic Algorithms

Figure 7.15 Cross section geometry for example problems.

Figure 7.16 Frame response optimized for $500 \mathrm{lb}$ crush response.

Figure 7.17 Frame response optimized for $1000 \mathrm{lb}$ crush force

Figure 7.18 Comparison to quasi isotropic design case.

Figure 7.19 Example for shape optimization.

Figure 7.20 Comparison of optimized $500 \mathrm{lb}$ case with and without shape optimization

Figure 7.21 Division of cross section into two shape regions.

Figure 7.22 Frame response optimized for $500 \mathrm{lb}$ crush response with 2 cross sections and one stacking sequence.

Figure 7.23 Modified penalty constraint

Figure 7.24 Frame response optimized for $500 \mathrm{lb}$ crush response with 2 cross-sectional geometries and one stacking sequence, with improved penalty.

Figure 7.25 Division of cross section into two shape regions.

Figure 7.26 Frame response optimized for $500 \mathrm{lb}$ crush response with 4 cross sections and one stacking sequence. 


\section{LIST OF TABLES}

Table 2.1: Active and reactive resultants and strains

Table 5.1 Deflection at the applied load from the finite element model of the arch shown in Figure 5.1

Table 5.2 Finite element performance for a straight isotropic angle section subjected to a distributed torsion

Table 5.3 Finite element performance for a straight isotropic I section subjected to a distributed torsion.

Table 5.4 Gjelsvik's parameter per unit length $\mu_{1} / L$ for the curved beam evaluation cases $(1 /$ in)

Table 5.5 Extensional stiffness matrix $[\mathrm{A}], \mathrm{lb} / \mathrm{in}$

Table 5.6 Coupling stiffness matrix $[\mathrm{B}], \mathrm{lb} / \mathrm{in}$

Table 5.7 Bending stiffness matrix $[\mathrm{D}], \mathrm{lb} / \mathrm{in}$

Table 5.8 Values $\mu_{1} / L$ for different $b / t$ ratios 108 
Table 5.9 Comparison of compliance ratios ("Beam Theory" / EAL branched shell) for the isotropic curved I section ( $R=36$ ", $b / t=17.85$ )

Table 5.10 Degrees of freedom used for E.A.L.shell analysis 109

Table 6.1 Empirical constants for lamina degradation factors 122

Table 6.2 Lamina degradation factors.

Table 6.3 Quasi isotropic coupon (NASA CR 4287) $\left.{ }^{49}\right], 8$ plies $[45 /-45 / 0 / 90]_{\mathrm{s}}$

Table 6.4 van Wamelen's ${ }^{[65]}$ on axis coupon, 16 plies $[0 / 90 / 45 / 0 / 90 /-45 / 90 / 0]_{s}$

Table 6.5 van Wamelen's ${ }^{[65]}$ off axis coupon, 16 plies $[10 / 100 / 55 / 10 / 100 /-35 / 100 / 10]_{s}$

Table 6.6 Material properties - BASF G30500/5208 graphite epoxy

Table 6.7 Tensile coupon laminate stacking sequences

Table 6.8 Failed ply and loads for progressive failure comparison to prediction by van Wamelen ${ }^{[65]}$ for on axis laminate tensile coupon

Table 6.9 Failed ply and loads for progressive failure comparison to prediction by van Wamelen ${ }^{[65]}$ for $10^{\circ}$ off axis laminate tensile coupon

Table 6.10 Material properties - AS4/5208 graphite epoxy

Table 6.11 Test frame laminate stacking sequences

Table 6.12 Branch lamina thicknesses for curved test frame.

Table 6.13 Energy absorbed as a function of mesh discretization 
Table 6.14 Influence of failure envelope on calculated energy absorption

Table 7.1 Ply Codes for laminate optimization

Table 7.2 Frame optimization example \#1

Table 7.3 Design improvement over baseline

Table 7.4 Additional $500 \mathrm{lb}$ designs for example \#1

Table 7.5 Additional $1000 \mathrm{lb}$ designs for example \#1

Table 7.6 Example of nodal input data for cross section shape.

Table 7.7 Shape coding

Table 7.8 Baseline cross section dimensions for shape optimization

Table 7.9 Energy absorbed for the $500 \mathrm{lb}$ case with shape optimization.

Table 7.10 Optimal cross section dimensions for the $500 \mathrm{lb}$ case, with shape optimization. 164

Table 7.11 Optimal designs obtained with shape optimization

Table $7.12500 \mathrm{lb}$ case with shape optimization and failure separation

Table 7.13 Optimal cross section shapes

Table $7.14500 \mathrm{lb}$ case with 2 cross-sectional geometries

Table 7.15 Optimal cross section shapes

Table 7.16.500 lb case with 4 cross-sectional geometries 
Table 7.17 Optimal cross section shapes

Table 7.18 Optimal cross section shapes

Table 8.1 Comparison of required solution time 


\section{CHAPTER 1}

\section{STRUCTURAL OPTIMIZATION FOR CRASHWORTHINESS}

\subsection{GENERAL PRINCIPLES OF CRASHWORTHY DESIGN}

The goal of crashworthy vehicle design is to increase occupant survivability and reduce the severity of injuries in crashes. The scope of research in this general area includes accident investigations, studies of human tolerance limits, investigations of seats and restraint systems, computer codes for crash simulation and failure analysis, full scale and component crash testing, development of energy absorbing structures and structural components, and the impact and use of new materials. General reviews of crashworthy design are given by Huculak ${ }^{[1]}$, and Poon ${ }^{[2]}$. Reviews of several major crashworthiness research programs are given by Farley et. al. ${ }^{[3]}$, Carden ${ }^{[4]}$, Hayduk et. al. ${ }^{[5]}$ and Thomson and Goetz ${ }^{[6]}$. From this research, five general design principles which lead to improved crashworthiness have been identified. ${ }^{[7]}$

1.) Limitoccupant accelerations - The vehicle structure must provide the necessary crash energy absorption to limit occupant accelerations to survivable levels.

2.) Maintain a survivable occupant volume- Under the conditions of an otherwise survivable crash, a survivable occupant volume should be maintained throughout the 
crash sequence.

3.) Retain high mass items - Massive items such as engines, galleys, service carts, stowage bins, etc., should be secured and supported sufficiently to prevent them from breaking loose and crashing into, or hurtling through the passenger compartment, thus causing injuries and fatalities.

4.) Minimize occupant environment hazards-Restraint systems and padding should be provided to prevent injury caused by occupant flailing and impact with adjacent items.

5.) Minimize postcrash hazards - Consideration must be given to flammability and release of toxic gases both during and after the crash sequence, as well as maintaining a usable exit from the vehicle.

By definition, survivable accidents are those in which the forces transmitted to the occupants due to abrupt accelerations do not exceed the limits of human tolerance ${ }^{[8]}$. The first and arguably the most important crashworthy design principle is: limit occupant accelerations to survivable levels by designing the vehicle structure for optimal crash energy absorption. This aspect of crashworthiness is viewed as critical since it is the first link in the chain of hazardous crash events. By designing the vehicle structure for optimal crash energy absorption, the envelope of survivable accidents is expanded and improved crashworthiness is the result. This work will focus on the objective of limiting occupant accelerations to survivable levels.

\subsection{STRUCTURAL REQUIREMENTS FOR CRASHWORTHINESS}

The structural design objective identified for crashworthiness is to protect vehicle occupants from exposure to high accelerations. A key to achieving such protection is controlled absorption of crash impact energy through progressive failure of the structure. By 
designing the vehicle to fail in a prescribed manner, the acceleration pulse of the crash event can be tailored for maximum survivability. This concept is illustrated in Figure 1.1

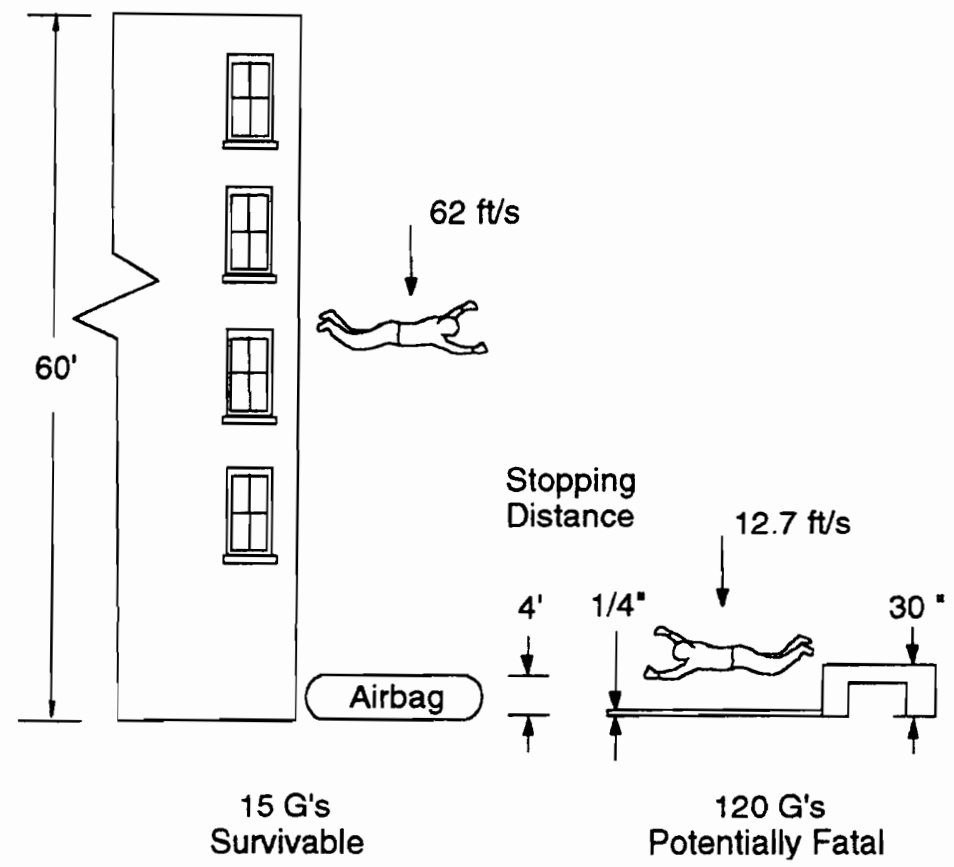

Figure 1.1 Tailoring impact accelerations for survivability

In this example, one individual leaps from a tall building and lands on a structure designed to absorb energy such as an airbag. Although the kinetic energy of the falling person is high, the air bag absorbs this energy through a slow progressive failure, and peak accelerations are limited to survivable levels. The other individual leaps from a desk to the floor. In this case, the kinetic energy at impact is much lower (approximately $1 / 20$ th), but the peak accelerations are eight times higher.This is because the essentially rigid floor absorbs the kinetic energy of the falling person relatively quickly, by stopping the vertical motion in a very short distance. This concept is expressed mathematically as follows. The kinetic energy of a moving object at impact is set equal to the energy required to stop its motion.

$$
\frac{1}{2} \mathrm{mV}^{2}=\int \mathrm{F}(\mathrm{x}) \mathrm{dx},
$$

where, $V$ is the impact velocity, $m$ is the mass of the falling object, and $F(x)$ is the force 
applied to stop its motion. Setting $F(x)=m \times a(x)$, where $a(x)$ is the acceleration as a function of $\mathrm{x}$, we obtain after some manipulation,

$$
a(x)=V \frac{d V}{d x}
$$

For a given impact velocity $\mathrm{V}$, the peak accelerations of Eq. (1.2) are a minimum when $\mathrm{a}(\mathrm{x})$ is a constant. Separating variables, both sides of $\mathrm{Eq}(1.2)$ can be integrated to obtain,

$$
\mathrm{A}=-\frac{\mathrm{V}^{2}}{2 \Delta \mathrm{x}}
$$

where $\mathrm{A}$ is the constant acceleration level, and $\Delta \mathrm{x}$ is the distance required to stop the objects motion. From Eq. (1.3), we see that for a given impact velocity V, the magnitude of the acceleration $\mathrm{A}$ is inversely related to the stopping distance $\Delta \mathrm{x}$. From this discussion, we can conclude that there are two important design requirements for maximizing crashworthiness.

1.) Maximizing structural capacity to absorb energy - The kinetic energy of the vehicle must be absorbed through a controlled progressive failure of the vehicle structure. The total area under the force displacement curve must equal the kinetic energy at impact-Eq.(1.1). If the capacity of the structure to absorb energy is not sufficient, the structure will "bottom out", and high and potentially fatal accelerations will be transmitted to the vehicle occupants. The required capacity for energy absorption is dictated by the severity of the design standard for crash impact conditions.

2.) Limiting the magnitude of peak accelerations -The allowable magnitude of the peak acceleration during progressive failure is constrained by the limits of human survival. Thus there is an upper bound on the acceleration $\mathrm{a}(\mathrm{x})$ and therefore on the failure force $F(x)$. The crashworthy structure must be designed for failure at or 
below a prescribed load. The magnitude of this failure load is dictated by the human tolerance limits for acceleration.

Maximizing crashworthiness in accordance with these design principles is equivalent to maximizing the area under the force-displacement failure response, subject to a constraint on maximum failure load. The maximum crashworthiness is obtained when the force-displacement curve has an elastic-plastic character, with the failure load determined by an upper bound on acceleration magnitude, and the failure displacement $\Delta \mathrm{x}$ (or stopping distance ) maximized. This type of failure response is illustrated in Figure 1.2.

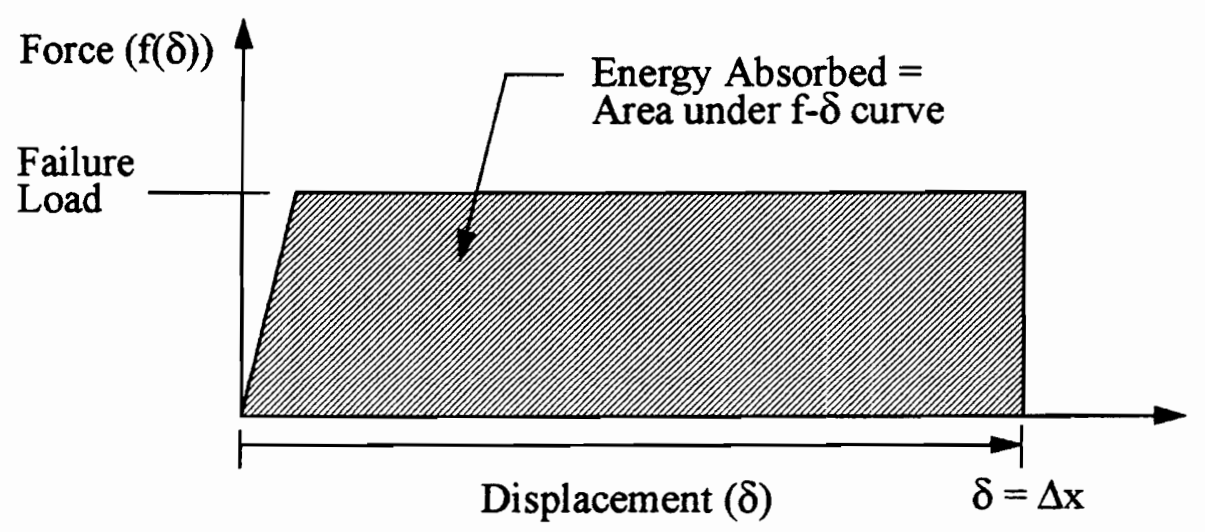

Figure 1.2 Optimal failure response

The concept of a design standard for crash severity is introduced with the first design requirement. The forces transmitted to vehicle occupants are heavily dependent on both vehicle design and crash severity. ${ }^{[9][10][11]}$ Crash impact conditions such as velocity (a vector), and attitude (pitch, roll, and yaw), are measures of crash severity. When applied to a specific vehicle, these conditions will determine the crash loads and occupant accelerations. Official standards for minimum crash resistance have been established for light fixed and rotary wing military aircraft ${ }^{[7]}$, and automobiles ${ }^{[12]}$, and have been proposed for civilian general aviation aircraft and small commercial aircraft. ${ }^{[13]}$ These standards normally specify a minimum probability of occupant survival for a range of impact conditions. By designing for maximum energy absorption, we are able to increase the 
survivable crash velocity or severity.

The focus of this work is crashworthiness of aircraft. Aircraft size, speed, configuration, and operational aspects all influence the crash environment so that no universal definition of a crash is possible. Studies of accident investigations reveal that a large percentage of accidents occur within a small range of flight paths and attitude ${ }^{[14][15][16]}$. The typical survivable crash for a transport category aircraft occurs near the airport at flight velocities below 150 knots, and vertical descent rates of less than $21 \mathrm{ft} / \mathrm{s}^{\left[{ }^{17]}\right.}$ Smaller aircraft and helicopters tend to crash at higher rates of vertical descent, and with lower forward velocityl $^{[11]}$. Because we know that certain categories of accidents occur more frequently for certain types of aircraft ${ }^{[17]}$, we can design for increased survivability in the most likely crash scenarios. A typical crash impact condition for an aircraft is one for which the attitude is relatively level and symmetric (e.g., $+15^{\circ}$ to $-5^{\circ}$ in pitch, $\pm 10^{\circ}$ in roll and yaw), but moving along a flight path that is both forward and downward. This situation is illustrated in Figure 1.3.

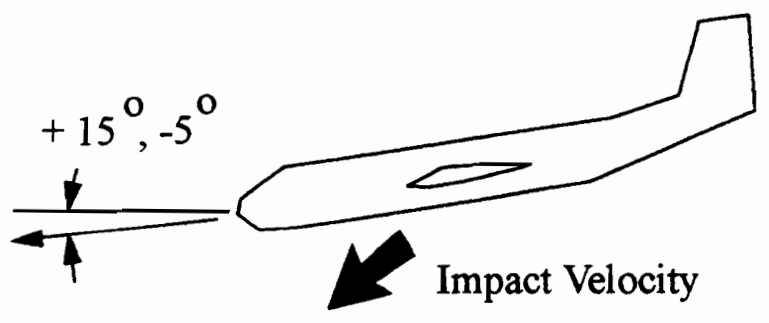

Figure 1.3 Typical crash scenario

The aircraft will not ordinarily stop forward motion immediately upon impact (unless it encounters a barrier ), but will slide (or plough) forward along the ground dissipating the forward component of kinetic energy. Vertical motion will stop relatively quickly upon impact. The vertical component of acceleration is of interest in the present work. Using 
the vehicle structure to absorb this vertical component of kinetic crash energy is critical to reducing the magnitude of the acceleration pulse transmitted to vehicle occupants.

\subsection{MECHANISMS FOR ABSORBING VERTICAL CRASH IMPACT ENERGY}

There are three principal structural mechanisms for absorption of vertical crash energy [11] :Stroking of the landing gear, crushing of the fuselage structure below the main passenger deck, and stroking of the seat support structure.(see Figure 1.4)

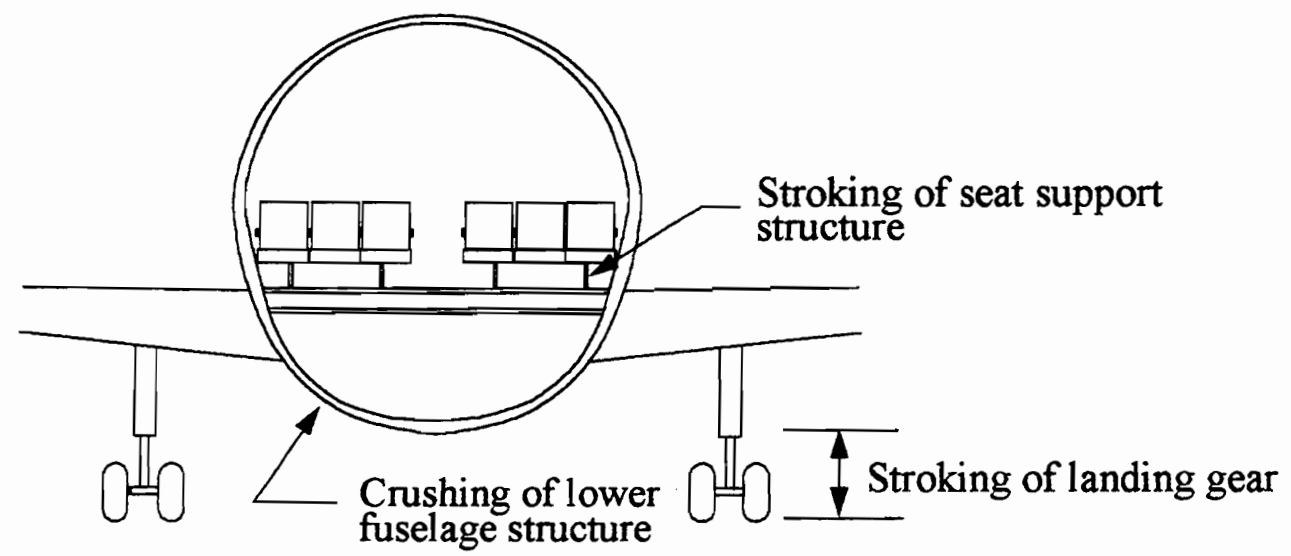

Figure 1.4 Major sources of vertical impact energy absorption

Of these three principal mechanisms, this work will focus on absorbing energy through controlled progressive crushing of the fuselage structure below the main passenger deck. Because fuselage frames are the major source of radial strength and stiffness in most aircraft fuselage structures, this failure mode is governed primarily by fuselage frame design. Ideally, load response of a frame will have the elastic-plastic character depicted in Figure 1.2. As dictated by the design requirements, we seek a controlled failure load followed by a sustained crushing force as a function of displacement at the point of contact. To achieve this, fuselage frames must be designed for an optimal crushing response. Frame design requirements established by the normal flight and service loads, are constrained by the additional requirements of a "potentially survivable" crash. 


\subsection{SPECIAL CONSIDERATIONS FOR COMPOSITE STRUCTURES}

Fuselage structures constructed from composite materials require a special attention to crashworthiness. ${ }^{[18]}$ It is known that properly designed composite components such as tubes loaded in compression can be excellent energy absorbers. ${ }^{\left[{ }^{19}\right]}$ They provide the relatively constant crushing force that is highly desired. When composite components are loaded transversely, as beams or frames, it is considerably more difficult to obtain an optimal crush response. [20][ 21 ][ 22]

Conventional aluminum skin-stringer fuselage designs will absorb kinetic crash energy through a process that involves ductile crushing and yielding of the fuselage structure. This type of crushing behavior is attributed to the formation of plastic hinges in the frames at the locations where local bending moments are high.

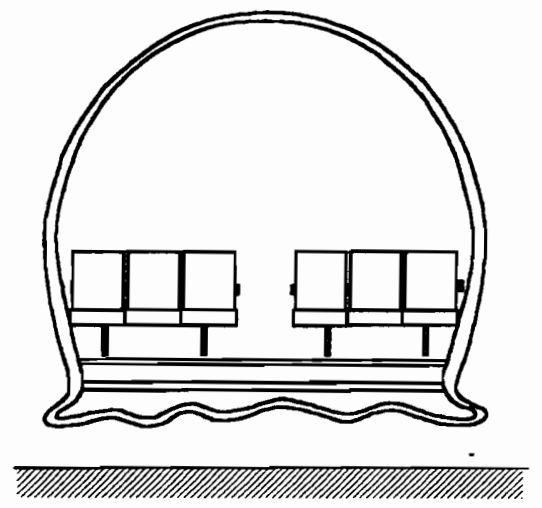

- Aluminum fuselage structures crush by ductile yielding of fuselage frames

Figure 1.5 Aluminum fuselage crushing behovior and energy absorption

For a laminated composite skin-stringer fuselage design, the thin walled frames are predisposed to fail by brittle fracture at these same locations. In this situation, tests by Carden et. al. ${ }^{[18]}$ show that the composite frames break into segments as depicted in Figure 1.6. Frame segments that effectively break free, are isolated from the main load path and are no longer involved in the process of energy absorption. Since less material is involved in the failure process, this brittle failure mode can result in a lowered energy absorption 
capability for composite frames.

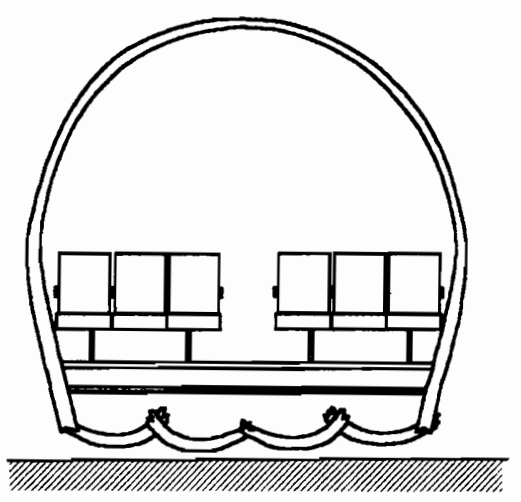

- Composite fuselage structures are predisposed to fail by brittle fracture.

- The frames break into segments involving less material in the failure process.

Figure 1.6 Composite fuselage crushing behavior and energy absorption

As a consequence of this brittle failure mode, designing composite fuselage structures to provide sufficient levels of crash energy absorption requires special attention. Maintaining or improving upon the existing levels of crashworthiness exhibited by conventional aluminum fuselage structures is an important design criterion for composite fuselage structures. Poor crashworthiness would adversely effect the widespread use and acceptance of these materials

\subsection{COMPOSITE FRAME DESIGN FOR CRASHWORTHINESS}

The typical aircraft has many fuselage frames. The function of these frames is to provide attachment points for internal and external mass items, radial strength for pressurization loads, and increased stability for the longerons and stringers. In a skin-stringer fuselage design, support provided by radial frame stiffness increases the structural stability of the longitudinal members, making it possible to decrease their size and weight.

Fuselage frames are typically designed as thin-walled curved beams with an open cross section. This type of frame has emerged as an aerospace design standard for valid reasons. The open cross-section facilitates attachments to the frame, while the thin-walled design minimizes weight. Curvature of the frame is normally established by aerodynamic or vehi- 
cle shape requirements. Material, and cross section geometry are the basic frame design variables, with ply orientation, and stacking sequence available as additional variables for laminated composite frames. These design parameters could be constant, or variable with angular position, and discontinuities or other trigger mechanisms such as drop plies, could be added to encourage specific failure modes. These traditional thin-walled open crosssection frame designs are the subject of this work.

For purposes of analysis for crashworthiness, we can isolate one frame ( possibly with some effective skin) from the fuselage structure and consider it independently. This is justified for two reasons; 1.) frames are the only major source of radially inward "crush" strength and stiffness in a fuselage, and 2.) there is little interaction between adjacent frames undergoing similar deformations. For an isolated frame, tests by Boitnott ${ }^{[23]}$ show that the top portion of the frame above the main passenger deck can also be ignored in an analysis of lower frame crush behavior. Therefore, in a design for crashworthiness, we can proceed by isolating and analyzing the lower portion of each frame independently as shown in Figure 1.7.

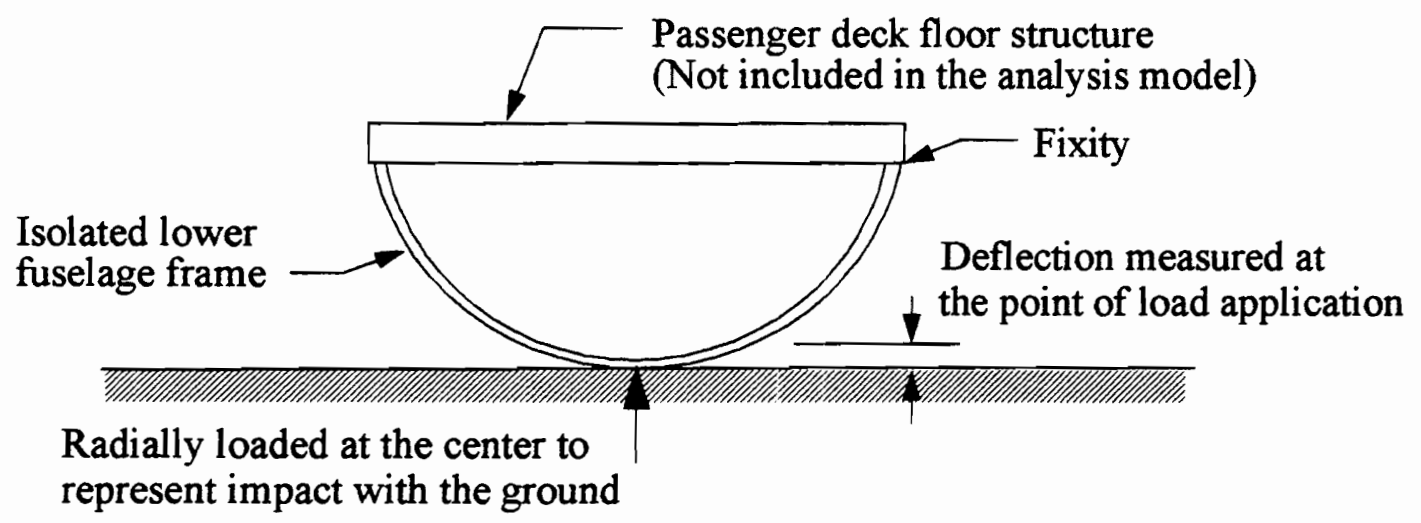

Figure 1.7 Analysis structure.

The task of analyzing composite frames for progressive failure is extremely challenging. Even the most analytically sophisticated attempts have not been entirely successful in accurately predicting the failure response of these structures. ${ }^{[24]}$ This is in part due to the 
complex nature of composite failure, and uncertainties in material properties, geometry, and boundary conditions. In some cases, the initiation and progression of failure in composite frames is governed by random defects in material and workmanship. Progressive failure is a sequence of events, where each event in the sequence is at least partially dependent upon the preceding events. A random variation in one failure event can result in an entirely different failure sequence. With this difficulty in mind, a goal in the present analysis is to capture the important features of the failure sequence that are relevant to preliminary design for crashworthiness. These features would include the peak failure load, and the total absorbed energy. A capability for exact progressive failure analysis is desirable, but is not considered to be a feasible option given the degree of analytical simplification mandated by the computational requirements of optimization

\subsection{OPTIMIZATION AND ANALYSIS FOR CRASHWORTHINESS}

Currently there are large gaps in the widely used analysis and design methodologies for crashworthiness. At one end of the spectrum are the highly analytical methods which rely on non-linear dynamic finite element analysis codes like DYNA3D ${ }^{25]}$, or DYCAST ${ }^{[26]}$ for progressive failure analysis. These codes are often computationally expensive, and require considerable expertise from their users. This expense, when combined with the difficult non-linear and discontinuous problem of optimizing composite structures for crashworthiness, makes the use of these purely analytical codes impractical for optimization in todays computing environment. At the other end of the spectrum are the semiempirical, or "hybrid" finite element progressive failure analysis codes like KRASH ${ }^{[27]}$. This class of analysis program relies on a combination of component progressive failure testing combined with dynamic finite element analysis to predict failure loads and accelerations. These programs are essentially a generalization of the lumped mass-spring concept to three dimensions. The structural response of each component is described by non-linear stiffness versus displacement curves. This requires that either detailed dynamic finite element analysis, or component testing be used to independently characterize the response of 
each element. A major disadvantage of this approach is that the failure response characterization is load dependent. Response characterization must be repeated for each anticipated loading configuration. The codes are appropriate when only the component loads and accelerations are required as output. Still another class of analysis lies in the middle ground between highly detailed finite elements, and the lumped mass-spring concept. Prior to the widespread availability of high speed computers, crush analysis, or collapse analysis was usually performed using simple elements with predefined failure mechanisms. In this method, the structural response of an element is modified when stresses reach a specified failure level. This usually involves placing one or more plastic hinges at appropriate places in the model. Applications to framed structures can be found in work by Majid ${ }^{28}$, Miles ${ }^{[29]}$, Toi and Yang[ ${ }^{[30]}$, and others. Murray[ ${ }^{[31]}$ and Mahmood and Paluszny ${ }^{[32]}$ adopt similar approaches to failure by adding a plastic hinge capability to shell elements. These shell elements are used to predict the failure response of thin walled beams modeled as a very coarse grid of shells. Methods that adapt to structural failure by adding plastic hinges work reasonably well for metallic structures which fail by ductile yielding, but plastic hinges may not be appropriate for the brittle failure modes of composite structures. These methods are still used because they are relatively efficient, and produce useful results for preliminary analysis and design.

Bolukbasi ${ }^{[33]}$ used the analysis program KRASH in conjunction with $\operatorname{CONMIN~}^{[34]}$ ( a popular optimization package ) to optimize the vertical crash response of a rotorcraft. The objective was to minimize the system weight increment associated with meeting the requirements for crash resistance subject to constraints on design and crash response parameters. A systems approach to energy absorption included as elements the landing gear, fuselage floor structure, and seats. Design parameters specified the load factors and maximum stroke for each of the three elements. Crash response parameters included occupant acceleration, velocity, and displacement time histories. An approximate model was used for the analysis. This model was built from a first-order sensitivity analysis of the crash response to each design parameter. The sensitivity analysis was calculated using 
KRASH, and had to be updated at intervals during the optimization process. Using separate load-response characterizations of seats, landing gear, and fuselage structures, a parametric weight analysis was developed. The basic flow of the solution was a KRASH based sensitivity analysis, followed by a CONMIN optimization cycle using the approximate analysis and the parametric weight analysis. This cycle was repeated until the optimum design was achieved. This approach does not design new energy absorbing structural components, but rather optimizes the system response using parameters that characterize an existing set of components.

In another application, Lust ${ }^{[35]}$ optimized the crash response of an automobile. The objective was to minimize weight subject to crash constraints, which included an upper bound on VCSI (Vertical Crash Severity Index which is an integral function of passenger acceleration). A one dimensional nonlinear spring-mass model was used in a two-phase analysis approach. First, the nonlinear behavior of each structural element was characterized using a separate analysis, then the spring-mass model determines the loads and accelerations. The integral constraint function was approximated locally for optimization efficiency. The optimization method was not specified, but was demonstrated by example to produce a more weight efficient crash design. This method was based on earlier work by Song ${ }^{[36]}$, who also used the two-phase methodology, and incorporated the separate analysis for calculating the nonlinear element stiffnesses. In the work by Song, beam element cross sectional dimensions were used as design variables. Because of the computational expense of calculating the beam response, a linear scaling approximation was used to estimate changes in the beam's load-response. These changes were based on small changes in design variables. CONMIN was used for optimization. The objective was to minimize weight subject to constraints placed on VCSI, and crush space. An example demonstrated a reduction in weight with all constraints satisfied with larger margins.

DeVries et. al. ${ }^{[37]}$. optimized thin walled beam structures for minimum weight subject to inertial, crush, and strength constraints. The method was based on the coupling of a beam 
section analysis code (SECOLLAPSE), with an optimizer (ADS ${ }^{[38}$ ) via Ford Motor Company's in-house structural optimization system OPUS. SECOLLAPSE computes a buckling strength, maximum strength, and mean crush strength for a thin walled beam in compression. For a beam in bending, an effective section property is calculated based on a buckling analysis of the beam flanges. The method was demonstrated by optimization of a rocker section profile for crash response.

In general, the previous optimization research has recognized the need for efficient analysis methods and approximations. Lust, Song, and DeVries use failure models with simple structural elements, and Bolukbasi uses a parameterization based on test and analysis. The models using simple adaptive elements have the advantage that optimization can be performed directly, using cross-section dimensions, etc. as design variables. All examples used some form of the hybrid lumped mass-spring analysis method. No examples were found that attempted to optimize crashworthiness using a DYNA3D type progressive failure analysis.

A method is needed to design composite fuselage frames for increased crashworthiness, and an efficient analysis method is considered essential to success. The existing adaptive models for collapse of metallic structures are not directly applicable to a composite structure because a brittle failure mode can cause discrete load drops at failure events. Excluding analysis done with programs like DYNA3D, progressive failure analysis for composite structures is usually done only at the level of simple components such as laminates (e.g. Tsai ${ }^{[39]}$, Petit and Waddoups ${ }^{[40]}$, Tolson and Zabaras ${ }^{[41]}$, Pandey and Reddy ${ }^{[42}$ I, and Chang and Chang ${ }^{[43]}$ ), and in these cases the mechanism of progressive failure is some form of lamina degradation model. Bolukbasi $\left.{ }^{44}\right]$ developed a methodology for predicting the progressive crushing load of composite stiffeners in compression based on an average failure load, local crippling, and buckling. In this example, the lamina failure loads of bias plies are used to calculate an average "Laminate Sustained Crush Stress" for 
thin walled composite columns. Simple design formulas are used to estimate laminate crippling and buckling. A more general adaptive progressive failure analysis method is required for optimization of curved composite frames. This work will focus on development of a progressive failure analysis for curved composite frames, and incorporating that analysis into an optimization program used for maximizing crashworthiness. The combination will become be a preliminary design methodology for curved composite frames.

\subsection{SCOPE AND OBJECTIVE}

The objective of this work is to develop a preliminary design methodology for optimizing the crashworthiness of composite aircraft fuselage frames. Structural optimization techniques typically rely on a structural analysis methodology for calculating the objective function and/or the constraints. With gradient based optimization methods, derivative information is also required. These calculations can be computationally expensive, particularly for progressive failure analysis. An efficient analysis method is considered essential to the ultimate success of a practical design methodology for optimizing crashworthiness.

The scope of this work covers five distinct areas. Concepts of crashworthiness were introduced and discussed in this chapter. These concepts define the optimization problem, and provide motivation for the present work. In Chapter 2, a thin-walled composite curved beam theory is developed. This thin-walled beam theory includes cross-sectional warping deformation due to torsion and is based on earlier work by Vlasov ${ }^{[5]}$. Modulus-weighted section properties in the material law for the beam theory require evaluation of many line integrals. Because of the complexity added by curvature, warping, and laminated composite material behavior, calculating these section properties is not trivial. It is an error prone process that was automated in this work by development of a section properties computer program. Section property computations are discussed separately in Chapter 3. In Chapter 4, a curved beam finite element is developed from the thin-walled, curved, composite beam theory developed in Chapter 2. Analysis with this element is performed using a 
finite element computer program developed as part of this work. The accuracy and limitations of the present beam theory and finite element analysis are discussed in Chapter 5 . Comparisons are made between the beam theory, and branched shell models, and discrepancies are discussed. The comparisons presented in Chapter 5 show that the finite element implementation of the thin-walled beam theory is a fast and reasonably accurate analysis method for the combined extensional, flexural, and torsional response of the frame. A progressive failure model for the thin-walled curved composite beam is presented and discussed in Chapter 6. This failure model is based on an extension of Tsai's ${ }^{[39]}$ selective and progressive ply-by-ply laminate degradation model. Implementation of the failure model is discussed, and a comparison to experimental data is presented. Optimization of curved frames for crashworthiness is discussed in Chapter 7. Genetic algorithms are used for the optimization process. The methodology developed in this work is shown to produce preliminary designs for composite frames with substantially increased crashworthiness. Concluding remarks are presented in Chapter 8. 


\section{CHAPTER 2}

\section{ANALYSIS OF THIN-WALLED LAMINATED COMPOSITE CURVED BEAMS}

\subsection{THEORY OF THIN-WALLED CURVED BEAMS}

Thin-walled beams are a unique category among easily identifiable basic structural elements. V.Z.Vlasov ${ }^{[45]}$, who is usually credited with the development of a combined theory for torsion and flexure of thin-walled open cross section beams, categorizes all structural elements into four general groups: massive bodies, plates and shells, solid beams, and thin-walled beams. From these categories, the thin-walled beams are distinguished as having three dimensions of different order of magnitude. They are viewed as built-up shell structures in which the thickness of the shell $(\delta)$ is small compared to a characteristic dimension of the cross-section(d), and the cross-sectional dimensions are small compared to the length of the shell(l). Vlasov suggested the following criterion as a means to identify thin walled beams:

$$
\frac{\delta}{d} \leq 0.1 \quad \frac{d}{\mathrm{l}} \leq 0.1
$$

The general category of thin-walled beams is further subdivided as open or closed crosssection, flexible or rigid cross section, and straight or curved beams. These classifications are a consequence of analytical differences in the approach to thin-walled beam problems. 
The theory developed here is applicable to thin-walled curved beams of rigid open crosssection

Thin walled beam theory calls for the introduction of terminology to designate elements of the beams cross section. The thin-walled beam is viewed as an assembly of shell units which form a structural element. For analysis purposes, the actual thin-walled beam crosssection is divided into discrete segments, where each segment of the cross-section is represented by a shell unit. To facilitate this division, terminology presented by Gjelsvik $\left.{ }^{[46}\right]$ is used to describe the elements of a beam cross-section. Corners and intersections in the cross section are referred to as junctions. A segment of the cross-section between two junctions, or between a junction and an end point is called a branch. Junctions are the boundaries between shell units, and each shell unit forms a branch of the beam cross-section. The line defined by the intersection of the middle surfaces of each shell unit with a plane normal to the circumferential axis of the beam is called the section contour line. The section contour line lies in the plane of the cross section, and locates the middle surface for each shell unit. The section contour line and the local wall thicknesses completely define the geometry of the beam cross-section. Simplifying assumptions regarding the shell strains of units in the beam cross-section are made with respect to the contour line. For this reason, the section contour line is an important concept for thin-walled beam theory. The terminology described is illustrated for a J-section in Figure 2.1.

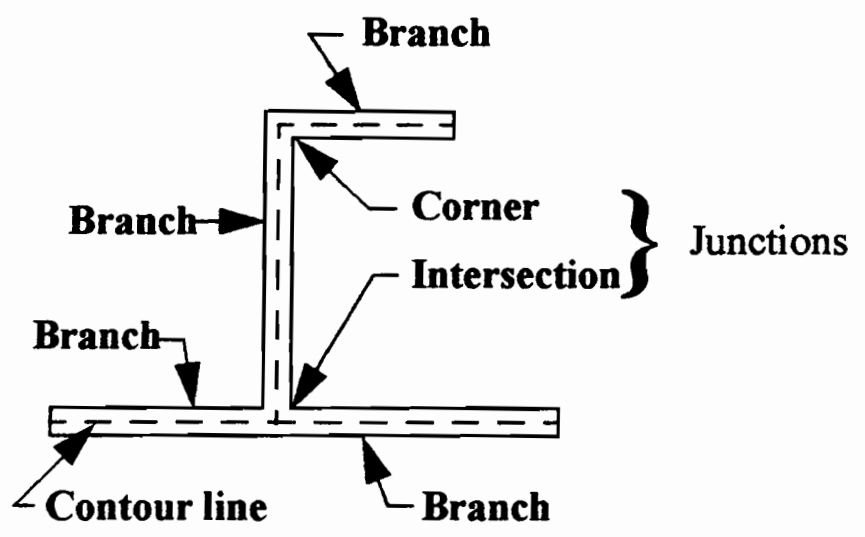

Figure 2.1 Cross section terminology 
The three major simplifying assumptions of thin-walled open cross section bar theory are (see also Gjelsvik ${ }^{[46]}$, p.6)

1.) The cross section remains rigid in its own plane.

2.) In-plane shear stiffness $A_{66} \rightarrow \infty$ such that the middle surface shear strain vanishes in each shell unit.

3.) Kirchhoff's hypotheses are valid for each shell unit. Normals to the shell middle surface remain straight and normal to the deformed reference surface, and do not change length.

The first assumption is used in most thin-walled bar theories (see the texts by Gjelsvik [46 ] and Murray ${ }^{[47]}$ for example). The second assumption was explicitly stated by Vlasov, and is sometimes referred to as the Vlasov assumption. This assumption leads to longitudinal displacements and strains along the contour as a result of torsional loads. More specifically, the Vlasov assumption leads to a displacement of the contour line out of the plane defined by flexure and extension of the bar. This additional longitudinal displacement is referred to as contour warping. The third assumption leads to an additional warping term which is defined for the longitudinal displacements of material points relative to the contour line. Referred to as thickness warping, this term accounts for shell strains present as a result of the bending and twisting deformations of each shell unit. Although thickness warping is neglected in some thin walled bar theories, Gjelsvik ${ }^{[46]}$ pp.69-74, shows that it can be the primary contribution to warping for some cross-sections. Total warping is the sum of the contributions from contour and thickness warping. Warping is a distinctive feature of thin walled beam theory.

In addition to the conventional bar forces and moments defined for the Bernoulli-Euler 
beam theory, a higher order axial stress resultant associated with warping is defined for thin-walled beams. This resultant is commonly referred to as the bimoment. It is statically equivalent to zero force, and zero moment. Typically, a bimoment is produced by longitudinal restraint of the ends of a thin-walled beam in torsion. An example of the bimoment applied to the end of a cantilever beam is illustrated in Figure 2.2.

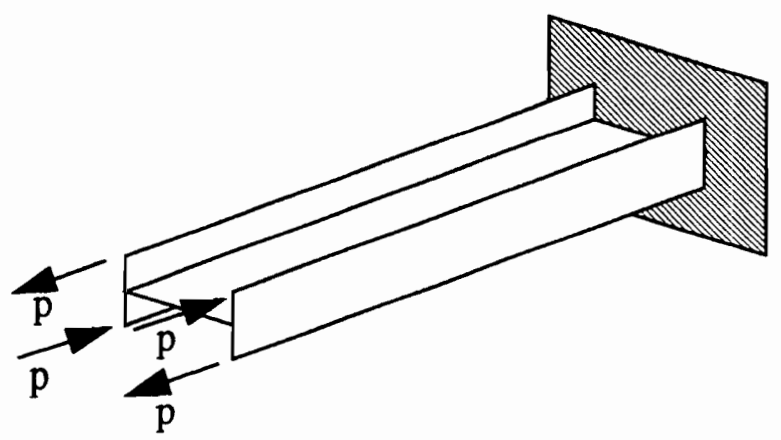

Figure 2.2 Example of the bimoment resultant

Because it is statically equivalent to zero force and zero moment, conventional beam theories often ignore the bimoment in accordance with Saint-Venant's principal. For thinwalled beams this assumption is not justified. The longitudinal normal stresses resulting from a bimoment decay very slowly along the length of a thin-walled beam. This is particularly true for laminated composite construction (see Choi and Horgan ${ }^{[48]}$ ). As a consequence of this slow decay rate, these stresses cannot be ignored in thin-walled beams with open cross-sections. Although the bimoment is statically equivalent to zero force and zero moment, the associated warping stresses in thin walled beams cannot in general be neglected by applying Saint-Venant's principle.

Vlasov's development of thin walled beam theory makes use of two special points in the cross-section in an effort to decouple the longitudinal, bending, and torsional modes of the deformation in the constitutive law. These two points correspond to the centroid and the shear center. For the analysis of generally laminated curved thin-walled beams, it is no 
longer possible to completely decouple the constitutive equations using these points. This is due to the coupling terms present as a result of curvature and material anisotropy. Furthermore, the effort to decouple the equations requires the use of two separate reference points for displacements and rotations of the cross-section. This unnecessarily complicates the finite element analysis by limiting the ability to offset the beam element centroid from the plane of curvature. Since decoupling these equations is generally not possible, and is of little benefit for the finite element analysis, the current development abandons the use of the special cross-section points in favor of a single arbitrary reference location about which all displacements and rotations are calculated. This formulation will result in a more generally applicable finite element for thin-walled curved laminated beams.

The basic thin-walled beam theory presented in this chapter follows the work originally done by Vlasov ${ }^{[45]}$ and later by Gjelsvik ${ }^{[46]}$. Collins and Johnson ${ }^{[49]}$ extended the theory to laminated curved beams using a method comparable to the current approach. The analysis developed here is applicable to laminated, curved, thin-walled beams of rigid open cross section. In the limit, as the radius of curvature of the beam approaches infinity, the current theory will reduce to the equivalent of Vlasov's straight thin-walled beam theory as extended to composite material wall construction by Bauld and Tzeng ${ }^{[50]}$. In this development, the strain energy relations for a curved thin-walled beam are formed based on Vlasov's theory. The theory is modified by establishing an arbitrary coordinate system origin, and by incorporating the constitutive relations of laminated composites into the expression of generalized Hooke's law for the thin-walled beam.

\subsection{COORDINATE SYSTEMS}

The theory for curved thin-walled beams is developed using three related coordinate systems; A cylindrical right-handed beam coordinate system, a right-handed cartesian crosssectional system, and a right-handed curvlinear shell coordinate system. These three coordinate systems are used to reduce the general three dimensional problem to a one dimen- 
sional curved beam theory. Shell coordinates are used to describe the displacements of material points in the cross-section relative to the cross-section contour. Cross-sectional coordinates are used to describe displacements of the section contour relative to the curved beam reference axis. The cylindrical beam coordinate system is used to descibe the displacements and rotations of the curved beam reference axis. Each coordinate system along with the associated coordinates are described in this section.

A cylindrical right-handed system with coordinates $(\theta, r, y)$ is used to describe the reference arc, or circumferential axis of the beam. This system is shown in Figure 2.3. The origin for this system is located at the center of curveature, and is designated " $O$ ". The $r-\theta$ plane defines the plane of curvature. The curved beam axis is defined along the circumferential or " $\theta$ " direction, with $\theta$ being defined as the circumferential beam coordinate. The curved beam segment extends from $\theta_{1}$ to $\theta_{2}$, with a radius of curvature " $r=a$ ".

The second system is a local cross-sectional Cartesian coordinate system defined relative to the curved beam's cylindrical system. It is a right-handed system with coordinates $(y, z$, $\mathrm{x}$ ). This system is used as a reference for the local displacements and rotations of the beam cross section. The $y$ - and z-axes form a local Cartesian system in the plane of the beam's cross section, with origin at the reference arc. This system is also shown in Figure 2.3. The two systems can be related as follows. The origin of the cross-sectional system is located at $" \mathrm{r}=\mathrm{a}$ " relative to the cylindrical system. The $\mathrm{x}$-axis lies in the plane of curvature tangent to the circumferential beam axis at $\theta$, the $z$-axis lies in the plane of curvature directed radially inward toward the center of curvature $O$, and the $y$-axis is in the transverse direction according to the right-hand rule. This relationship can be expressed as $d x=(a) d \theta, r=$ 
$\mathrm{a}-\mathrm{z}$, and $\mathrm{y}=\mathrm{y}$.

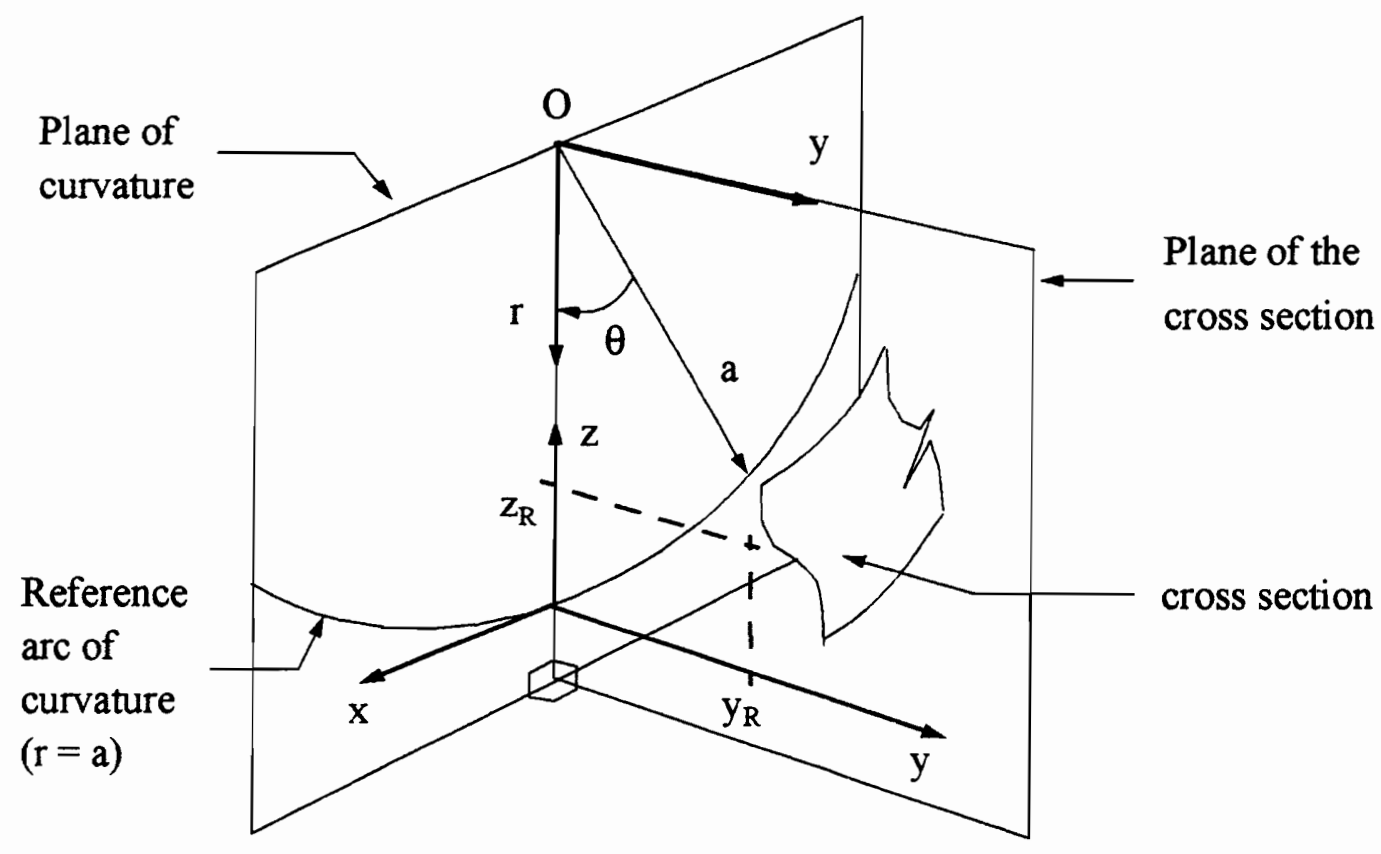

Figure 2.3 Cylindrical and cross section coordinates.

The third coordinate system is referred to as the shell coordinate system. This is a righthanded curvilinear system defined in terms of the section contour. Recall that the section contour is a line defined by the intersection of the middle surfaces of each shell unit with a plane normal to the circumferential axis of the beam. The coordinates for this system are $(\zeta, s, x)$. The direction for arc-length coordinate $s$ is defined as tangent to the section contour, $x$ is aligned parallel to the longitudinal $x$-axis of the cross sectional system, and $\zeta$ is in the plane of the contour, normal to the tangent, with the positive sense defined by the 
right hand rule. This system and its relation to the $(y, z, x)$ system is shown in Figure 2.4.

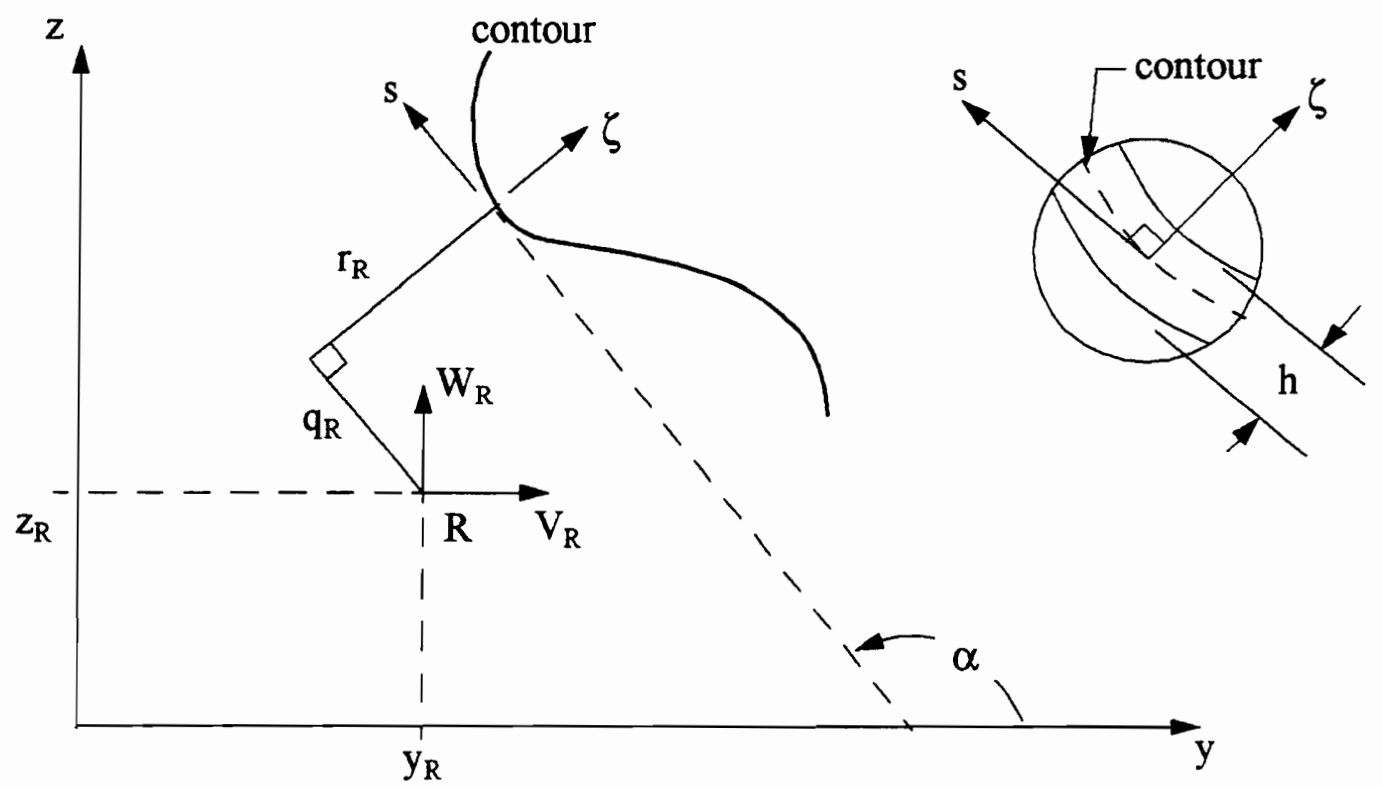

Figure 2.4 Contour coordinates and sign convention.

With this definition, $\zeta$ becomes a shell thickness coordinate measured normal to the tangent of the contour at $\mathrm{s}$. On the section contour, $\zeta=0$. If $h$ is defined as the thickness of the shell at $\mathrm{s}$, then $|\zeta|<=\mathrm{h} / 2$. This follows from the previous definition of the contour line as being located at the middle wall thickness for each shell. In general, the shell thickness $\mathrm{h}$ can be a function of $s$. The $(\zeta, s, x)$ coordinate system is rotated by an angle $\left(\alpha-90^{\circ}\right)$ with respect to the $(y, z, x)$ system. The angle $\alpha$ is defined as the angle between the positive $y$ axis, and the positive s direction at a generic point on the contour. Positive $\alpha$ is measured counterclockwise when viewed down the positive $\mathrm{x}$-axis.

\subsection{COORDINATE RELATIONS}

Let $\bar{y}(s)$ and $\bar{z}(s)$ designate the $(y, z)$ coordinates of a point on the section contour at $s$. These coordinates satisfy the following differential relations 


$$
\frac{d \bar{y}}{d s}=\cos \alpha, \quad \frac{d \bar{z}}{d s}=\sin \alpha
$$

A material point at shell coordinates $(\zeta, s)$ in the wall of a cross-sectional element can now be described in terms of $(y, z)$ coordinates by.

$$
\begin{aligned}
& y(\zeta, s)=\bar{y}(s)+\zeta \sin \alpha(s) \\
& z(\zeta, s)=\bar{z}(s)-\zeta \cos \alpha(s)
\end{aligned}
$$

Using Eqs. (2.2)-(2.4) the following derivatives are obtained for later use.

$$
\begin{aligned}
& \mathrm{y}_{{ }_{\mathrm{s}}}=\left(1+\frac{\zeta}{\mathrm{R}_{\mathrm{s}}}\right) \cos \alpha \mathrm{z}_{{ }_{\mathrm{s}}}=\left(1+\frac{\zeta}{\mathrm{R}_{\mathrm{s}}}\right) \sin \alpha \\
& \mathrm{y}_{{ }_{\zeta}}=\sin \alpha \quad \mathrm{z}_{{ }_{\zeta}}=-\cos \alpha
\end{aligned}
$$

where partial differentiation with respect to a coordinate is denoted by a comma with the coordinate following as a subscript, $1 / R_{s}=d \alpha / d s$, and $R_{s}$ is the radius of curvature of the section contour in the $y-z$ plane.

Contour coordinates $r_{R}$, and $\mathrm{q}_{R}$ are introduced as shown in Figure 2.4. These are coordinates in the $\zeta$ and $s$ directions respectively, of a point on the contour relative to a reference point $R$. Where $R$ is an arbitrary location in the plane of the cross-section about which all displacements and rotations are referenced. The location of $R$ in the $(y, z, x)$ coordinate system is designated $\left(y_{R}, z_{R}\right)$, where the $x$ coordinate has been dropped for the analysis of a particular cross-section. The direction of positive $r_{R}$ is defined by the positive direction of $\zeta$, and the direction of positive $q_{R}$ is defined by the positive direction of $s$. Parameters $r_{R}$ and $q_{R}$ can be positive or negative in value.

The contour coordinates at neighboring points are related. Consider a position vector $\overline{\mathrm{RA}}$ 
directed from point $\mathrm{R}$ to a generic point $\mathrm{A}$ at $\mathrm{s}$ on the contour as shown in Figure 2.5.

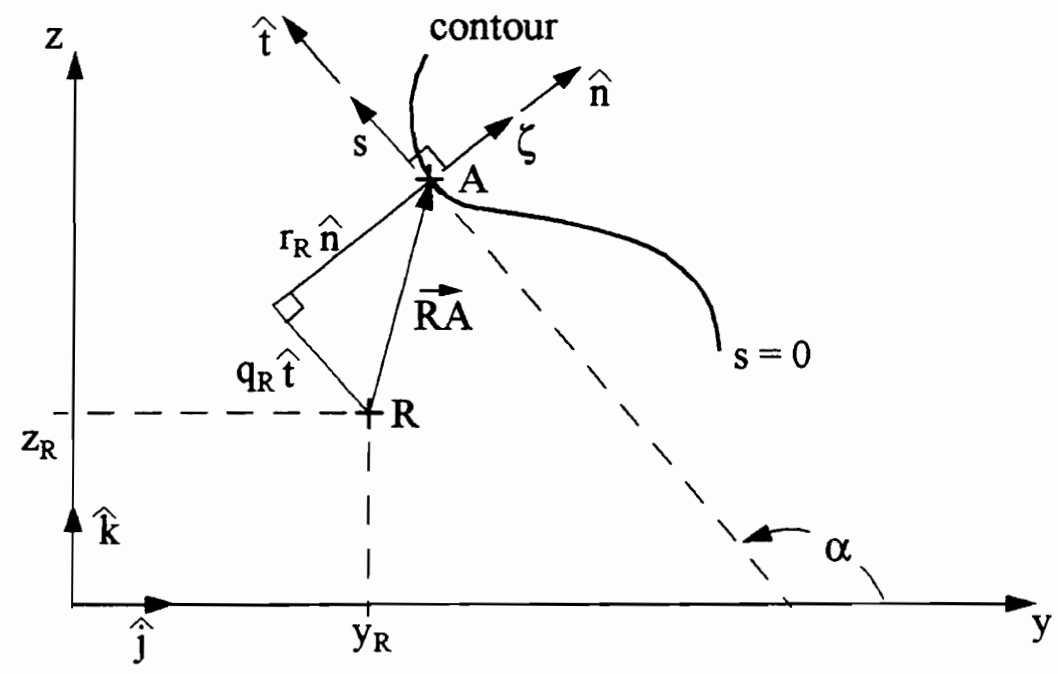

Figure 2.5 Relationship between contour coordinates

In contour coordinates this relative position vector is

$$
\overline{R A}=q_{R} \hat{t}+r_{R} \hat{n}
$$

where $\hat{\mathrm{t}}$ is a unit tangent vector to the contour, positive in the sense of increasing $\mathrm{s}$, and $\hat{\mathrm{n}}$ is a unit normal vector to the contour, positive in the positive $\zeta$ direction. The derivatives of these unit vectors with respect to s are expressed in terms of $\alpha$ using the chain rule as

$$
\begin{array}{ll}
\frac{\mathrm{d} \hat{\mathrm{t}}}{\mathrm{ds}}=\frac{\mathrm{d} \hat{\mathrm{t}}}{\mathrm{d} \alpha} \frac{\mathrm{d} \alpha}{\mathrm{ds}}=\frac{\mathrm{d} \hat{\mathrm{t}}}{\mathrm{d} \alpha} \frac{1}{\mathrm{R}_{\mathrm{s}}} & \text { where, } \frac{\mathrm{d} \hat{\mathrm{t}}}{\mathrm{d} \alpha}=-\hat{\mathrm{n}} \\
\frac{\mathrm{d} \hat{\mathrm{n}}}{\mathrm{ds}}=\frac{\mathrm{d} \hat{\mathrm{n}}}{\mathrm{d} \alpha} \frac{\mathrm{d} \alpha}{\mathrm{ds}}=\frac{\mathrm{d} \hat{\mathrm{n}}}{\mathrm{d} \alpha} \frac{1}{\mathrm{R}_{\mathrm{s}}} & \text { where, } \frac{\mathrm{d} \hat{\mathrm{n}}}{\mathrm{d} \alpha}=\hat{\mathrm{t}}
\end{array}
$$

The derivative of $\overline{\mathrm{RA}}$ can be written as 


$$
\frac{d \overline{R A}}{d s}=\left(\frac{d q_{R}}{d s}+\frac{r_{R}}{R_{s}}\right) \hat{t}+\left(\frac{d r_{R}}{d s}-\frac{q_{R}}{R_{s}}\right) \hat{n}
$$

but along the contour $\frac{\mathrm{d} \overline{\mathrm{RA}}}{\mathrm{ds}}=\mathfrak{t}$, so that

$$
\begin{aligned}
& \frac{\mathrm{dq}_{\mathrm{R}}}{\mathrm{ds}}+\frac{\mathrm{r}_{\mathrm{R}}}{\mathrm{R}_{\mathrm{s}}}=1 \\
& \frac{\mathrm{dr}_{\mathrm{R}}}{\mathrm{ds}}-\frac{\mathrm{q}_{\mathrm{R}}}{\mathrm{R}_{\mathrm{s}}}=0
\end{aligned}
$$

The position vector from the reference point to a generic material point in the cross section in the contour coordinates is

$$
\overline{\mathrm{R}}=\mathrm{q}_{\mathrm{R}} \mathfrak{\mathrm { t }}+\left(\mathrm{r}_{\mathrm{R}}+\zeta\right) \hat{\mathrm{n}}
$$

In the $y$ - and $\mathrm{z}$ - coordinates this same vector is

$$
\bar{R}=\left(y-y_{R}\right) \hat{\jmath}+\left(z-z_{R}\right) \hat{k}
$$

where $\hat{j}$, and $\hat{k}$ are unit vectors in the positive $y$ - and $z$-directions, respectively. Equating these two representations of $\bar{R}$, and noting $\hat{\mathrm{t}} \cdot \hat{\mathrm{j}}=-\hat{\mathrm{n}} \cdot \hat{\mathrm{k}}=\cos \alpha$, and

$$
\begin{aligned}
\hat{\mathfrak{t}} \cdot \hat{k}= & \hat{\mathrm{n}} \cdot \hat{\mathrm{j}}=\sin \alpha \text {, we get } \\
& \mathrm{r}_{\mathrm{R}}(\mathrm{s})+\zeta=\left(y-y_{\mathrm{R}}\right) \sin (\alpha)-\left(z-z_{\mathrm{R}}\right) \cos (\alpha) \\
& \mathrm{q}_{\mathrm{R}}(\mathrm{s})=\left(\mathrm{y}-\mathrm{y}_{\mathrm{R}}\right) \cos (\alpha)+\left(z-z_{R}\right) \sin (\alpha)
\end{aligned}
$$

The inverted form for these equations is 


$$
\begin{aligned}
& \left(y-y_{R}\right)=\left(r_{R}(s)+\zeta\right) \sin (\alpha)+q_{R}(s) \cos (\alpha) \\
& \left(z-z_{R}\right)=-\left(r_{R}(s)+\zeta\right) \cos (\alpha)+q_{R}(s) \sin (\alpha)
\end{aligned}
$$

The sign convention described for the contour coordinates $r_{R}$, and $q_{R}$, must be carefully followed. These coordinates are central to the theory of thin walled beams.

\section{4 KINEMATICS OF DEFORMATION}

Let $\bar{u}_{R}(\theta)=U_{R}(\theta) \hat{i}(\theta)+V_{R}(\theta) \hat{\jmath}+W_{R}(\theta) \hat{k}(\theta)$ be the displacement of reference point $R$, where $U_{R}(\theta)$ is the circumferential displacement of $R, V_{R}(\theta)$ is the y-direction displacement of $R$, and $W_{R}(\theta)$ is the $z$-direction displacement of $R$. Unit vectors $\hat{i}, \hat{j}$, and $\bar{k}$ are functions of $\theta$ such that

$$
\frac{\mathrm{d} \hat{\mathrm{i}}}{\mathrm{d} \theta}=\mathrm{k}, \quad \frac{\mathrm{d} \hat{\mathrm{j}}}{\mathrm{d} \theta}=0, \quad \frac{\mathrm{d} \hat{\mathrm{k}}}{\mathrm{d} \theta}=-\hat{\mathrm{i}} \quad,
$$

The position vector of point $\mathrm{R}$ relative to point $\mathrm{O}$ before deformation is

$$
\overline{\mathbf{R}}_{\mathrm{R}}=-\left(\mathbf{a}-\mathrm{z}_{\mathrm{R}}\right) \hat{\mathrm{k}}+\mathrm{y}_{\mathrm{R}} \hat{\mathrm{j}},
$$

The position of $\mathbf{R}$ in the deformed beam, denoted, $\mathbf{R}^{*}$, is given by the position vector

$$
\overline{\mathrm{R}}_{\mathrm{R}}^{*}=\overline{\mathrm{R}}_{\mathrm{R}}+\overline{\mathrm{u}}_{\mathrm{R}}(\theta)
$$

An infinitesimal line element parallel to the $\mathrm{x}$-axis ( or $\theta$-direction) in the deformed ring is

$$
\begin{aligned}
& d \bar{R}_{R}=-\left(a-z_{R}\right)(-\hat{i} d \theta)=d s_{R} \hat{\imath} \\
& \text { where ds } s_{R}=\left(a-z_{R}\right) d \theta
\end{aligned}
$$


Infinitesimal vector $d \bar{R}_{R}$ is mapped to $d \bar{R}_{R}{ }^{*}$ in the deformed configuration by

$$
\mathrm{d}_{\mathrm{R}}{ }_{\mathrm{R}}^{*}=\mathrm{d} \overline{\mathrm{R}}_{\mathrm{R}}+\mathrm{d} \overline{\mathrm{u}}_{\mathrm{R}}
$$

In component form

$$
d \bar{R}_{R}^{*}=d s_{R} \hat{\imath}+\left[\left(U_{R}^{\prime}-W_{R}\right) \hat{\imath}+V_{R}^{\prime} \hat{\jmath}+\left(W_{R}^{\prime}+U_{R}\right) k\right] d \theta
$$

where prime means ()$^{\prime}=d() / d \theta$. Rearranging, we get

$$
\mathrm{dR}_{\mathrm{R}}{ }^{*}=\left\{\left[1+\frac{\left(\mathrm{U}_{\mathrm{R}}^{\prime}-\mathrm{W}_{\mathrm{R}}\right)}{\mathrm{a}-\mathrm{z}_{\mathrm{R}}}\right] \hat{\imath}+\left[\frac{\mathrm{V}_{\mathrm{R}}^{\prime}}{\mathrm{a}-\mathrm{z}_{\mathrm{R}}}\right] \hat{\jmath}+\left[\frac{\mathrm{W}_{\mathrm{R}}^{\prime}+\mathrm{U}_{\mathrm{R}}}{\mathrm{a}-\mathrm{z}_{\mathrm{R}}}\right] \mathrm{k}\right\} \mathrm{ds} \mathrm{s}_{\mathrm{R}}
$$

The new infinitesimal length is $\left(\mathrm{ds}_{\mathrm{R}}{ }^{*}\right)^{2}=\mathrm{d \textrm {R } _ { \mathrm { R } }}{ }^{*} \bullet \mathrm{d} \overline{\mathrm{R}}_{\mathrm{R}}{ }^{*}$, which is written as

$$
\mathrm{ds}_{\mathrm{R}}^{*}=\lambda_{\mathrm{R}} \mathrm{ds} \mathrm{R}_{\mathrm{R}}
$$

where $\lambda_{\mathrm{R}}$ is the stretch ratio, identified as

$$
\lambda_{R}^{2}=\left[1+\frac{\left(U_{R}^{\prime}-W_{R}\right)}{a-z_{R}}\right]^{2}+\left[\frac{V_{R}^{\prime}}{a-z_{R}}\right]^{2}+\left[\frac{W_{R}^{\prime}+U_{R}}{a-z_{R}}\right]^{2}
$$

A unit vector tangent to the reference line element in the deformed ring is

$$
\hat{1}^{*}=\frac{\mathrm{dR}_{\mathrm{R}}^{*}}{\mathrm{ds}_{\mathrm{R}}^{*}}
$$

which is written as

$$
\hat{\imath}^{*}=1_{11} \hat{\imath}+1_{12} \hat{\jmath}+1_{13} \hat{k}
$$


where the direction cosines are defined by

$$
\begin{aligned}
& 1_{11}=\frac{1}{\lambda_{R}}\left[1+\frac{\left(U_{R}^{\prime}-W_{R}\right)}{a-z_{R}}\right] \\
& 1_{12}=\frac{1}{\lambda_{R}}\left[\frac{V_{R}^{\prime}}{a-z_{R}}\right] \\
& 1_{13}=\frac{1}{\lambda_{R}}\left[\frac{W_{R}^{\prime}+U_{R}}{a-z_{R}}\right]
\end{aligned}
$$

such that $\quad 1_{11}^{2}+1_{12}^{2}+1_{13}^{2}=1$

It is assumed that plane cross sections of the ring first displace and rotate to a plane normal to the deformed reference axis of the ring, called the flexural plane. Second, the flexural plane can rotate as a rigid body about the reference axis; call this plane the flexural-torsional plane. Third, the material points in the cross section are permitted to displace normal to the flexural-torsional plane - a warping of the cross section.

The flexural plane is defined solely on the basis of the rotation of a line element $d \bar{R}_{R}$ to $\mathrm{d} \overline{\mathbf{R}}_{\mathrm{R}}{ }^{*}$, or by unit vectors $\hat{i}$ to $\hat{i}{ }^{*}$. Removing the translational part, we can let points $\mathbf{R}^{*}$ and $R$ coincide. Let $\beta$ denote the angle of rotation from $\hat{i}$ to $\hat{1}^{*}$. The cross product rule for these two vectors gives

$$
\hat{\imath} \times \hat{\imath}^{*}=\sin (\beta) \hat{\mathrm{e}},
$$

where $\hat{e}$ is a unit vector perpendicular to the plane of $\hat{\imath}$ and $\hat{\imath}^{*}$, and defines an axis of rotation due to bending. Hence

$$
\sin (\beta) \hat{\mathrm{e}}=-1_{13} \hat{\jmath}+1_{12} \hat{\mathrm{K}},
$$




$$
\sin ^{2}(\beta)=1_{13}^{2}+1_{12}^{2}=1-1_{11}^{2},
$$

also

$$
\cos (\beta)=1_{11} \quad\left(\hat{\imath} \cdot \hat{1}^{*}=\cos (\beta)\right)
$$

Therefore, a unit vector along the axis of rotation is

$$
\hat{\mathbf{e}}=\frac{1_{13}}{\sqrt{1_{12}^{2}+1_{13}^{2}}} \hat{\jmath}+\frac{1_{12}}{\sqrt{1_{12}^{2}+1_{13}^{2}}} \hat{k},
$$

When the local coordinate system is rotated by an angle $\beta$ with respect to the axis $\hat{\mathbf{e}}$, the new vector representation of an arbitrary vector $\bar{r}$, which is fixed on the local coordinate system, can be expressed as

$$
\overline{\mathrm{r}}_{1}=(1-\cos (\beta))(\overline{\mathrm{r}} \cdot \hat{\mathrm{e}}) \hat{\mathrm{e}}+\cos (\beta) \overline{\mathrm{r}}+\sin (\beta) \hat{\mathrm{e}} \times \overline{\mathrm{r}},
$$

This rotation is shown in Figure 2.6.

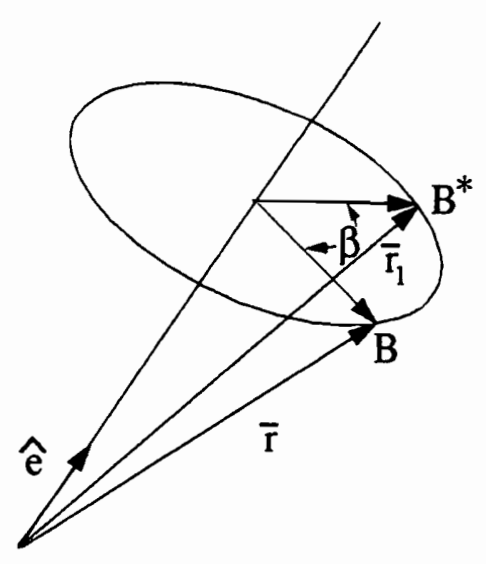

Figure 2.6 Rotation of position vector $\mathrm{r}$ to $\overline{\mathrm{r}}_{1}$ through angle $\beta$ about the cxis of rotation $\hat{\mathrm{e}}$. 
Substituting $\overline{\mathrm{r}}=\hat{\mathrm{i}}$ and, $\overline{\mathrm{r}}_{1}=\hat{\mathrm{i}}_{1}, \overline{\mathrm{r}}=\hat{\mathrm{j}}$ and, $\overline{\mathrm{r}}_{1}=\hat{\mathrm{j}}_{1}, \overline{\mathrm{r}}=\hat{\mathrm{k}}$ and, $\overline{\mathrm{r}}_{1}=\hat{\mathrm{k}}_{1}$ into this rotation equation, we get

$$
\left\{\begin{array}{l}
\hat{\mathrm{i}}_{1} \\
\hat{\mathrm{j}}_{1} \\
\hat{\mathrm{k}}_{1}
\end{array}\right\}=\left[\begin{array}{ccc}
1_{11} & 1_{12} & 1_{13} \\
-1_{12} & 1_{11}+\frac{1_{13}^{2}}{\left(1+1_{11}\right)} & -\frac{1_{12} 1_{13}}{\left(1+1_{11}\right)} \\
-1_{13} & -\frac{1_{12} 1_{13}}{\left(1+l_{11}\right)} & 1_{11}+\frac{1_{13}^{2}}{\left(1+1_{11}\right)}
\end{array}\right]\left\{\begin{array}{l}
\hat{\imath} \\
\hat{\jmath} \\
\hat{k}
\end{array}\right\}
$$

Clearly $\hat{i}_{1}=\hat{i}^{*}$. The mutually orthogonal unit vectors $\hat{i}_{1}, \hat{j}_{1}$, and $\hat{k}_{1}$, are defined by rigid body rotation through the angle $\beta$ about the axis $\hat{e}$. Unit vectors $\hat{j}_{1}$ and $\hat{k}_{1}$ lie in the flexural plane of the deformed beam. (Note that when $\beta=0,1_{11}=1,1_{12}=1_{13}=0$, so $\quad \hat{i}_{1}=\hat{\imath}, \hat{j}_{1}=\hat{\jmath}$, and $\left.\hat{k}_{1}=\hat{k} \quad\right)$

The flexural-torsional plane is defined as a rotation $\Phi_{x R}$, about axis $\hat{i}_{1}$

$$
\left\{\begin{array}{l}
\hat{\mathrm{i}}^{*} \\
\hat{\mathrm{j}}^{*} \\
\mathrm{k}^{*}
\end{array}\right\}=\left[\begin{array}{ccc}
1 & 0 & 0 \\
0 & \cos \left(\Phi_{\mathrm{xR}}\right) & \sin \left(\Phi_{\mathrm{xR}}\right) \\
0 & -\sin \left(\Phi_{\mathrm{xR}}\right) & \cos \left(\Phi_{\mathrm{xR}}\right)
\end{array}\right]\left\{\begin{array}{c}
\hat{\mathrm{i}}_{1} \\
\hat{\mathrm{j}}_{1} \\
\hat{\mathrm{k}}_{1}
\end{array}\right\}
$$

Clearly the flexural plane and the flexural-torsional plane coincide, and the latter only distinguishes the final positions of unit vectors $\hat{j}^{*}$, and $\mathrm{k}^{*}$, with respect to unit vectors $\hat{\mathrm{j}}_{1}$, and $\hat{\mathrm{k}}_{1}$. 
The total rotation of the cross section, which is normal to the $x$-axis in the undeformed ring, and normal to the reference axis in the deformed ring, is provided by the composite of the rotations of $\beta$ (flexure) first, then $\Phi_{\mathrm{xR}}$ (torsion) second; i.e.,

$$
\left\{\begin{array}{l}
\hat{\imath}^{*} \\
\hat{\jmath}^{*} \\
\mathrm{k}^{*}
\end{array}\right\}=\left[\mathrm{R}_{2}\left(\Phi_{\mathrm{xR}}\right)\right]\left[\mathrm{R}_{1}(\boldsymbol{\beta})\right]\left\{\begin{array}{l}
\hat{\mathrm{i}} \\
\hat{\mathrm{j}} \\
\mathrm{k}
\end{array}\right\}
$$

where

$$
\begin{aligned}
& \mathbf{R}_{2}\left(\Phi_{x R}\right)=\left[\begin{array}{ccc}
1 & 0 & 0 \\
0 & \cos \left(\Phi_{x R}\right) & \sin \left(\Phi_{x R}\right) \\
0 & -\sin \left(\Phi_{x R}\right) & \cos \left(\Phi_{x R}\right)
\end{array}\right] \\
& R_{1}(\beta)=\left[\begin{array}{ccc}
111 & 1_{12} & 1_{13} \\
-1_{12} & 1_{11}+\frac{1_{13}}{\left(1+1_{11}\right)} & -\frac{1_{12} 1_{13}}{\left(1+1_{11}\right)} \\
-1_{13} & -\frac{1_{12} 1_{13}}{\left(1+1_{11}\right)} & 1_{11}+\frac{1_{13}^{2}}{\left(1+1_{11}\right)}
\end{array}\right]
\end{aligned}
$$


An example which illustrates these displacements and rotations is shown in Figure 2.7,

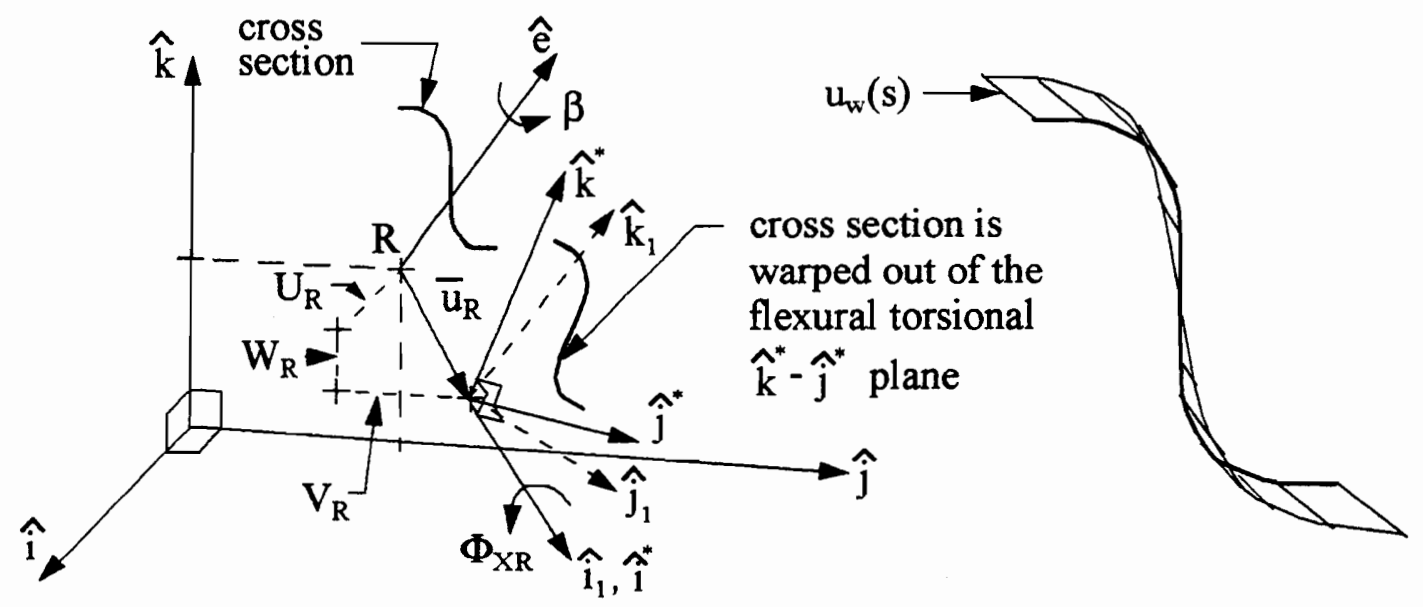

Figure 2.7 Displacements of the beam cross section

Let $\overline{\mathrm{u}}(\mathrm{y}, \mathrm{z}, \boldsymbol{\theta})$ denote the displacement of a generic material point $\mathrm{A}$. The displacement of $A$ to its final position is separated into three parts.
a) translation determined by $R \rightarrow R^{*}$.
b) rotations $\beta$ and $\Phi_{x R}$ to position $A_{F}$ in the flexural plane.
c) warping displacement $u_{w}$ normal to flexural plane.

Thus, $\overline{\mathrm{u}}(\mathrm{y}, \mathrm{z}, \theta)=\overline{\mathrm{u}}_{\mathrm{R}}(\theta)+\overline{\mathrm{R}^{*} \mathrm{~A}_{\mathrm{F}}}-\overline{\mathrm{RA}}+\mathrm{u}_{\mathrm{w}} \hat{\mathrm{1}}^{*}$,

with

$$
\begin{aligned}
& \overline{R A}=\left(y-y_{R}\right) \hat{\jmath}+\left(z-z_{R}\right) \hat{k} \\
& \overline{R^{*} A_{F}}=\left(y-y_{R}\right) \hat{j *}+\left(z-z_{R}\right) \hat{k^{*}}
\end{aligned}
$$


and $|\overline{\mathrm{RA}}|=\left|\overline{\mathrm{R}^{*} \mathrm{~A}_{\mathrm{F}}}\right|$ since the flexural plane is rigid. The displacement is written as

$$
\overline{\mathrm{u}}(\mathrm{y}, \mathrm{z}, \theta)=\overline{\mathrm{u}}_{\mathrm{R}}(\theta)+\left(\mathrm{y}-\mathrm{y}_{\mathrm{R}}\right)\left(\hat{j}^{*}-\hat{\mathrm{j}}\right)-\left(\mathrm{z}-\mathrm{z}_{\mathrm{R}}\right)\left(\hat{\mathrm{k}}^{*}-\mathrm{k}\right)+\mathrm{u}_{\mathrm{w}} \hat{\mathrm{i}}^{*}
$$

Up to this point in the derivation, all algebra is exact. Assumptions are only made with respect to the kinematic degrees of freedom. For infinitesimal rotations and displacements, the magnitude of displacement gradients for the reference line element are all assumed to be small with respect to unity; i.e.,

$$
\begin{aligned}
& 0<\left|\frac{\left(\mathrm{U}_{\mathrm{R}}-\mathrm{W}_{\mathrm{R}}\right)}{\mathrm{a}-\mathrm{z}_{\mathrm{R}}}\right| \ll 1 \\
& 0<\left|\frac{\mathrm{V}_{\mathrm{R}}^{\prime}}{\mathrm{a}-\mathrm{z}_{\mathrm{R}}}\right| \ll 1 \\
& 0<\left|\frac{\mathrm{W}_{\mathrm{R}}^{\prime}+\mathrm{U}_{\mathrm{R}}}{\mathrm{a}-\mathrm{z}_{\mathrm{R}}}\right| \ll 1 \\
& 0<\left|\Phi_{\mathrm{XR}}\right| \ll 1
\end{aligned}
$$

For this assumption, $\lambda_{\mathrm{R}} \cong 1$, and the direction cosines become

$$
l_{11} \cong 1, \quad l_{12} \cong \frac{V_{R}^{\prime}}{a-z_{R}}, \quad l_{13} \cong \frac{W_{R}^{\prime}+U_{R}}{a-z_{R}} \quad,
$$

The direction cosine $1_{12} \cong \Phi_{\mathrm{zR}}$, where $\Phi_{\mathrm{zR}}$ is the rotation about the z-axis of the projection of $\hat{i^{*}}$ into the $x-y$ plane; ie,

$$
\tan \left(\Phi_{\mathrm{zR}}\right)=\frac{1_{12}}{\Gamma_{11}}
$$

Consequently, for infinitesimal rotation 


$$
\Phi_{\mathrm{zR}} \cong \frac{1_{12}}{1}=\frac{\mathrm{V}_{\mathrm{R}}^{\prime}}{\mathrm{a}-\mathrm{z}_{\mathrm{R}}}
$$

The direction cosine $1_{13} \cong \Phi_{\mathrm{yR}}$, where $\Phi_{\mathrm{yR}}$ is the rotation about the y-axis of the projection of $\hat{i^{*}}$ into the $x-z$ plane; ie.,

$$
\tan \left(-\Phi_{\mathrm{yR}}\right)=\frac{1_{13}}{1_{11}}
$$

and for infinitestimal rotation

$$
\Phi_{\mathrm{yR}} \cong-\frac{1_{13}}{1}=\frac{\mathrm{W}_{\mathrm{R}}^{\prime}+\mathrm{U}_{\mathrm{R}}}{\mathrm{a}-\mathrm{z}_{\mathrm{R}}}
$$

Substituting these approximations for infinitesimal rotations into the rotation matrices $R_{1}$, and $R_{2}$, and neglecting product terms like $\Phi_{y R} \Phi_{z R}$, the total rotation is

$$
\left\{\begin{array}{c}
\hat{\mathrm{i}}^{*} \\
\hat{\mathrm{j}}^{*} \\
\mathrm{k}^{*}
\end{array}\right\}=\left[\begin{array}{ccc}
1 & \Phi_{\mathrm{zR}} & -\Phi_{\mathrm{yR}} \\
-\Phi_{\mathrm{zR}} & 1 & \Phi_{\mathrm{xR}} \\
\Phi_{\mathrm{yR}} & -\Phi_{\mathrm{xR}} & 1
\end{array}\right]\left\{\begin{array}{l}
\hat{\mathrm{i}} \\
\hat{\mathrm{j}} \\
\hat{\mathrm{k}}
\end{array}\right\}
$$

The displacements of a generic point in the cross section are now related to the displacements and rotations about the reference point $R$ by

$$
\begin{aligned}
\overline{\mathrm{u}}(\mathrm{y}, \mathrm{z}, \theta)= & \overline{\mathrm{u}}_{\mathrm{R}}(\theta)+\left(\mathrm{y}-\mathrm{y}_{\mathrm{R}}\right)\left[-\Phi_{\mathrm{zR}}(\theta) \hat{\mathrm{i}}+\Phi_{\mathrm{xR}}(\theta) \mathrm{k}\right] \\
& +\left(\mathrm{z}-\mathrm{z}_{\mathrm{R}}\right)\left[\Phi_{\mathrm{yR}}(\theta) \hat{\mathrm{i}}-\Phi_{\mathrm{xR}}(\theta) \hat{\jmath}\right]-\omega(\zeta, \mathrm{s}) \tau(\theta) \hat{\mathrm{i}}
\end{aligned}
$$

where the warping term $u_{w}$ is assumed to be in a separable form and $\hat{i}^{*} \cong i$ for infinitestimal rotations; ie 


$$
u_{w}(y, z, \theta) \hat{\imath}^{*} \cong-\omega(\zeta, s) \tau(\theta) \hat{\imath}
$$

Now designate the $\mathrm{y}, \mathrm{z}$ and $\theta$ displacement components of the generic point $\mathrm{A}$ as $\mathrm{v}, \mathrm{w}$, and $\mathrm{u}$ respectively. Because these displacements are a function of position along the curved beam segment, it is convenient to replace the cross-sectional $\mathrm{x}$ coordinate by $\theta$. The displacement components are determined from Eq.(2.17) and are given by

$$
\begin{aligned}
\mathrm{u}(\mathrm{y}, \mathrm{z}, \theta)= & \mathrm{U}_{\mathrm{R}}(\theta)-\left(\mathrm{y}-\mathrm{y}_{\mathrm{R}}\right) \Phi_{\mathrm{zR}}(\theta) \\
& +\left(\mathrm{z}-\mathrm{z}_{\mathrm{R}}\right) \Phi_{\mathrm{yR}}(\theta)-\omega(\zeta, \mathrm{s}) \tau(\theta) \\
\mathrm{v}(\mathrm{y}, \mathrm{z}, \theta)= & \mathrm{V}_{\mathrm{R}}(\theta)-\left(\mathrm{z}-\mathrm{z}_{\mathrm{R}}\right) \Phi_{\mathrm{xR}}(\theta) \\
\mathrm{w}(\mathrm{y}, \mathrm{z}, \theta)= & \mathrm{w}_{\mathrm{R}}(\theta)+\left(\mathrm{y}-\mathrm{y}_{\mathrm{R}}\right) \Phi_{\mathrm{yR}}(\theta)
\end{aligned}
$$

where $U_{R}(\theta)$ is the circumferential displacement of the reference point $R, V_{R}(\theta)$ is the $y$ direction displacement of $R$, and $W_{R}(\theta)$ is the z-direction displacement of $R$. Quantities $\Phi_{\mathrm{yR}}, \Phi_{\mathrm{zR}}$ and $\Phi_{\mathrm{xR}}$ are rotations (assumed to be infinitesimal), (see Eqs.(2.15) and (2.16)) about axes passing through the point $R$, which are parallel to the $y_{-}^{-}, z_{-}$, and $x_{-}^{-}$, axes respectively. These displacements and rotations are shown in Figure 2.8. Eqs.(2.18)-(2.20) represent assumptions with respect to the kinematics of thin walled curved beams. They are equivalent to the kinematic assumptions made by Vlasov ${ }^{[45}$, and Gjelsvik ${ }^{[46]}$. Expressions for the warping displacement $u_{w}=-\omega(\zeta, s) \tau(\theta)$ are determined from the straindisplacement equations and the Vlasov and Kirchhoff assumptions. The strain-displace- 
ment relations are discussed in the next section.

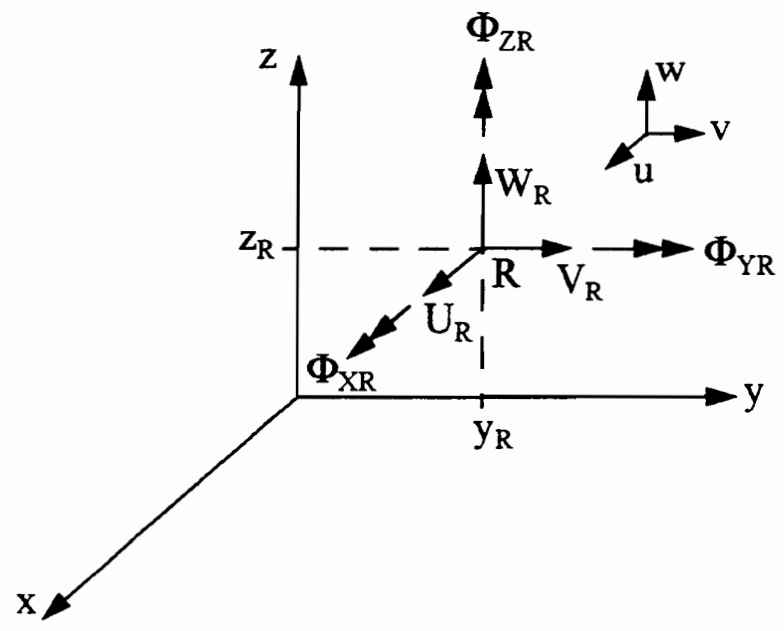

Figure 2.8 Bar displacements and rotations referenced to point $R$

\subsection{STRAIN-DISPLACEMENT}

In cylindrical coordinates the linear strain displacement equations are

$$
\begin{aligned}
& \varepsilon_{\theta \theta}=\left(\mathrm{u}_{\theta}-\mathrm{w}\right) /(\mathrm{a}-\mathrm{z}) \\
& \varepsilon_{\mathrm{yy}}=\mathrm{v}, \mathrm{y} \\
& \varepsilon_{\mathrm{zz}}=\mathrm{w}, \mathrm{z} \\
& \gamma_{\theta y}=\mathrm{u},{ }_{\mathrm{y}}+\left(\mathrm{v},{ }_{\theta}\right) /(\mathrm{a}-\mathrm{z}) \\
& \gamma_{\theta z}=\mathrm{u},{ }_{\mathrm{z}}+\left(\mathrm{w},{ }_{\theta}+\mathrm{u}\right) /(\mathrm{a}-\mathrm{z}) \\
& \gamma_{\mathrm{yz}}=\mathrm{v},{ }_{\mathrm{z}}+\mathrm{w}, \mathrm{y}
\end{aligned}
$$

where the radial coordinate $r$ has been replaced by $(a-z)$.

Substituting the displacements of Eqs.(2.18)-(2.20) into the strains of Eqs. (2.21) results in

$$
\begin{aligned}
(\mathrm{a}-\mathrm{z}) \varepsilon_{\theta \theta}= & U_{\mathrm{R}}^{\prime}-\mathrm{W}_{\mathrm{R}}-\left(\mathrm{y}-\mathrm{y}_{\mathrm{R}}\right)\left(\Phi_{\mathrm{zR}}^{\prime}+\Phi_{\mathrm{xR}}\right) \\
& +\left(\mathrm{z}-\mathrm{z}_{\mathrm{R}}\right) \Phi_{\mathrm{yR}}^{\prime}-\omega(\zeta, \mathrm{s}) \tau^{\prime}
\end{aligned}
$$




$$
\begin{aligned}
\varepsilon_{y y}=\varepsilon_{z z}= & 0 \\
(a-z) \gamma_{\theta y}= & (a-z)\left(-\Phi_{z R}-\omega, y\right)+V_{R}^{\prime}-\left(z-z_{R}\right) \Phi_{x R}^{\prime} \\
(a-z) \gamma_{\theta z}= & (a-z)\left(\Phi_{y R}-\omega, \tau\right)+W_{R}^{\prime}+\left(y-y_{R}\right) \Phi_{x R}^{\prime}+U_{R} \\
& -\left(y-y_{R}\right) \Phi_{z R}+\left(z-z_{R}\right) \Phi_{y R}-\omega \tau \\
\gamma_{y z}=0 &
\end{aligned}
$$

where a prime represents an ordinary derivative with respect to $\theta$. Vanishing of the strains in Eqs. (2.23), and (2.26) verifies that there is no distortion of the cross-section. This satisfies the first assumption that the cross section remains rigid in its own plane.

The unknown warping function $\omega(\zeta, s)$ is obtained by applying assumptions 2 (Vlasov) and 3 (Kirchhoff) of the current theory to the expressions for transverse shear strain given by Eqs.(2.24), and (2.25). Substituting Eqs.(2.15) and (2.16) into Eqs.(2.24) and (2.25), results in

$$
\begin{aligned}
& (a-z) \gamma_{\theta y}=-\left(z-z_{R}\right)\left(\Phi_{x R}^{\prime}-\Phi_{z R}\right)-[(a-z) \omega, y] \tau(\theta) \\
& (a-z) \gamma_{\theta z}=-\left(y-y_{R}\right)\left(\Phi_{x R}^{\prime}-\Phi_{z R}\right)-[(a-z) \omega, z+\omega] \tau(\theta)
\end{aligned}
$$

Since assumptions 2 , and 3 are associated with shear strain quantities in the shell coordinate system, it is necessary to transform the shear strains given by Eqs.(2.27), and (2.28) to the shell system prior to applying these assumptions. The transverse shear strain components in the shell coordinates $(\zeta, \mathrm{s}, \mathrm{x})$ are $\gamma_{\theta \mathrm{s}}$, and $\gamma_{\theta \zeta}$. They are given by the transformation equations

$$
\begin{aligned}
& \gamma_{\theta s}=\gamma_{\theta y} \cos \alpha+\gamma_{\theta z} \sin \alpha \\
& \gamma_{\theta \zeta}=\gamma_{\theta y} \sin \alpha-\gamma_{\theta z} \cos \alpha
\end{aligned}
$$

Substituting Eqs. (2.27) and (2.28) into Eqs. (2.29) and (2.30) gives 


$$
\begin{aligned}
& (\mathrm{a}-\mathrm{z}) \gamma_{\theta \mathrm{s}}=\left(\Phi_{\mathrm{xR}}^{\prime}-\Phi_{\mathrm{zR}}\right)\left[\left(\mathrm{y}-\mathrm{y}_{\mathrm{R}}\right) \sin \alpha-\left(\mathrm{z}-\mathrm{z}_{\mathrm{R}}\right) \cos \alpha\right] \\
& +\left[-(a-z)\left(\omega, y \cos \alpha+\omega, z^{\sin \alpha)-\omega \sin \alpha] \tau}\right.\right. \\
& (\mathrm{a}-\mathrm{z}) \gamma_{\theta \zeta}=-\left(\Phi_{\mathrm{xR}}^{\prime}-\Phi_{\mathrm{zR}}\right)\left[\left(\mathrm{y}-\mathrm{y}_{\mathrm{R}}\right) \cos \alpha+\left(\mathrm{z}-\mathrm{z}_{\mathrm{R}}\right) \sin \alpha\right] \\
& +[-(a-z)(\omega, y \sin \alpha-\omega, z \cos \alpha)+\omega \cos \alpha] \tau
\end{aligned}
$$

From the chain rule and Eqs.(2.5) and (2.6), partial derivatives of the warping function in shell coordinates are

$$
\begin{aligned}
& \omega,_{s}=\left(1+\zeta / R_{s}\right)\left(\omega, y \cos \alpha+\omega, z^{\sin \alpha}\right) \\
& \omega, \zeta=\omega, y \sin \alpha-\omega, z^{\cos \alpha}
\end{aligned}
$$

Substitution of Eqs. (2.11), (2.12), (2.33) and (2.34) into Eqs. (2.31), and (2.32) gives the transverse shear strains in terms of contour coordinates as

$$
\begin{aligned}
(a-z) \gamma_{\theta s}= & \left(\Phi^{\prime}{ }_{x R}-\Phi_{z R}\right)\left(r_{R}+\zeta\right) \\
& -\left((a-z)\left(1+\zeta / R_{s}\right)^{-1} \omega, s+\omega \sin \alpha\right) \tau \\
(a-z) \gamma_{\theta \zeta}= & -\left(\Phi^{\prime}{ }_{x R}-\Phi_{z R}\right) q_{R}-((a-z) \omega, \zeta-\omega \cos \alpha) \tau
\end{aligned}
$$

Using Eqs. (2.5) and (2.6) it can be shown that

$$
\begin{aligned}
& \frac{(a-z)^{2}}{\left(1+\zeta / R_{s}\right)} \frac{\partial}{\partial s}\left(\frac{\omega}{a-z}\right)=(a-z)\left(1+\zeta / R_{s}\right)^{-1} \omega, s+\omega \sin \alpha \\
& (a-z)^{2} \frac{\partial}{\partial \zeta}\left(\frac{\omega}{a-z}\right)=(a-z) \omega, \zeta-\omega \cos \alpha
\end{aligned}
$$

Using Eqs. (2.37) and (2.38), Eqs. (2.35) and (2.36) can be rewritten as

$$
(a-z) \gamma_{\theta s}=\left(\Phi_{X R}^{\prime}-\Phi_{z R}\right)\left(r_{R}(s)+\zeta\right)-\left[\frac{(a-z)^{2}}{\left(1+\zeta / R_{s}\right.} \frac{\partial}{\partial s}\left(\frac{\omega}{a-z}\right)\right] \tau
$$




$$
(a-z) \gamma_{\theta \zeta}=-\left(\Phi_{x R}^{\prime}-\Phi_{z R}\right) q_{R}-\left[(a-z)^{2} \frac{\partial}{\partial \zeta}\left(\frac{\omega}{a-z}\right)\right] \tau
$$

Vlasov's assumption is that the reference surface shear strains vanish. This means that $\gamma_{\theta s}(\zeta=0, s)=0$. Setting $\omega(\zeta=0, s)=\bar{\omega}(\mathrm{s})$ in Eq. $(2.39)$, the Vlasov assumption implies that

$$
\left(\Phi_{x R}^{\prime}(\theta)-\Phi_{z R}(\theta)\right) r_{R}(s)-\left[(a-\bar{z}(s))^{2} \frac{d}{d s}\left(\frac{\bar{\omega}(s)}{a-\bar{z}(s)}\right)\right] \tau(\theta)=0
$$

The two terms in Eq. (2.41) consist of a product of a function of $\theta$, times a function of $s$. We can satisfy this equation by choosing,

$$
\tau(\theta)=\frac{\left(\Phi_{\mathrm{xR}}^{\prime}-\Phi_{\mathrm{zR}}\right)}{\left(\mathrm{a}-\mathrm{z}_{\mathrm{R}}\right)}
$$

so that,

$$
\left(a-z_{R}\right) r_{R}(s)-(a-\bar{z}(s))^{2} \frac{d}{d s}\left(\frac{\bar{\omega}(s)}{a-\bar{z}(s)}\right)=0
$$

We note that for infinitesimal rotations, a rotation vector can be defined as

$$
\vec{\Omega}(\theta)=\Phi_{\mathrm{XR}}(\theta) \hat{i}(\theta)+\Phi_{\mathrm{YR}}(\theta) \hat{\jmath}(\theta)+\Phi_{\mathrm{ZR}}(\theta) \mathrm{K}(\theta)
$$

The gradient of this vector with respect to the reference axis arc length is

$$
\frac{1}{\left(\mathrm{a}-\mathrm{Z}_{\mathrm{R}}\right)} \frac{\mathrm{d} \overrightarrow{\boldsymbol{\Omega}}}{\mathrm{d} \boldsymbol{\theta}}=\frac{1}{\left(\mathrm{a}-\mathrm{z}_{\mathrm{R}}\right)}\left[\left(\boldsymbol{\Phi}_{\mathrm{XR}}^{\prime}-\boldsymbol{\Phi}_{\mathrm{ZR}}\right) \hat{\mathrm{\imath}}+\boldsymbol{\Phi}_{\mathrm{YR}}^{\prime} \hat{\jmath}+\left(\Phi_{\mathrm{ZR}}^{\prime}+\boldsymbol{\Phi}_{\mathrm{XR}}\right) \hat{\mathrm{k}}\right]
$$

The definition of $\tau(\theta)$, or rate of twist, in Eq.(2.42) is the $\hat{\imath}$-direction component of the rotation gradient vector. 
Integrating Eq. (2.43) with respect to $s$, from $s=0$ to $s$, we obtain

$$
\bar{\omega}(s)-\bar{\omega}(0)=\left(a-z_{R}\right)(a-\bar{z}(s)) \int_{0}^{s} \frac{r_{R}(s)}{(a-\bar{z}(s))^{2}} d s
$$

Function $\bar{\omega}(s)$ is known as the contour warping function. It specifies the cross sectional distribution of the additional axial displacements of the contour line which are a result of torsion. These additional axial displacements due to torsion are referred to as warping.

Because the displacements must be continuous at the junctions, $\bar{\omega}$ (s) must also be continuous at these junctions. In practice, the sense of coordinate $s$ is arbitrary along the contour line in each branch, where each branch in the cross section is taken in an arbitrary order. Furthermore, the origin for $s$ is arbitrary. After specifying the sequence of branches, the direction along each branch, and the origin for $s$, continuity for $\bar{\omega}(s)$ can be established. Continuity at the junctions is imposed by introducing and evaluating constants for each branch function. Note that even though $\bar{\omega}(s)$ is continuous at the junctions, $\bar{\omega}(s)$ is not in general a continuous function of $\mathbf{s}$. After continuity at the junctions is established, the origin for $\mathrm{s}$ can be selected such that the integral in Eq. (2.44) vanishes if the integration is performed over the entire contour $\mathrm{C}$. This origin for $\mathrm{s}$ is referred to by Vlasov as the principal contour origin. The principal origin is useful in decoupling the torsional equations for some types of thin-walled beams. There may be one origin for $s$, more than one, or an entire branch on the contour for which this integral vanishes. In this case any such point can be considered the principal contour origin. The principal contour origin is considered a cross-sectional property.

As the radius of curvature (a), becomes large, then Eq. (2.44) reduces to 


$$
\bar{\omega}(s)=\int_{0}^{s} r_{R}(s) d s
$$

which is the same expression obtained for straight beam theory.

The warping function has a geometrical interpretation. To appreciate this, let $\overline{\mathrm{RA}}$ represent a vector from the reference point $R$, to a point $A$ located on the contour (see Figure 2.9). The contour warping function $\bar{\omega}$ (s) of Eq. (2.45) represents twice the area swept out by the radius vector $\overline{\mathrm{RA}}$, as $\mathrm{A}$ moves along the contour from $\mathrm{s}=0$ to $\mathrm{s}$.

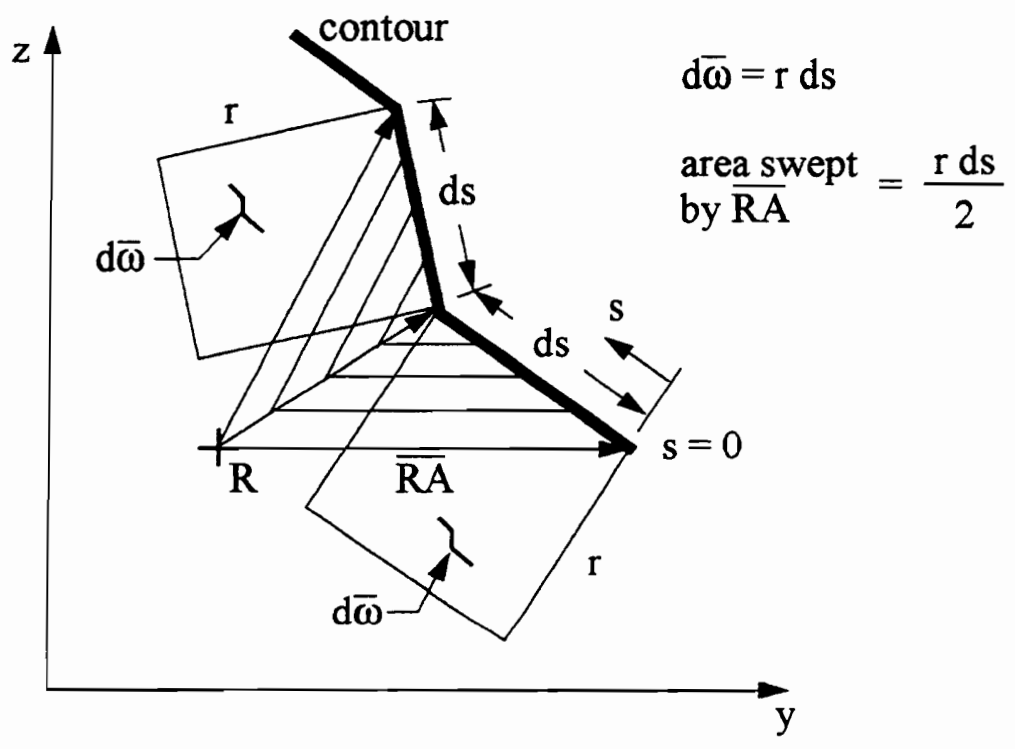

Figure 2.9 Contour warping function

For this reason function $\bar{\omega}$ is sometimes referred to as the sectorial area, and $R$ is referred to as the pole of sectorial areas. The location chosen for $R$ affects the value of the warping function $\bar{\omega}$. Since $\bar{\omega}$ determines the distribution of axial strain as a result of torsion, moving $\mathbf{R}$ in the $y$ direction is equivalent to flexure about an axis through the contour origin, parallel to the $z$-axis. Likewise, moving $R$ in the $z$ direction is equivalent to flexure about 
an axis through the contour origin parallel to the $y$-axis. For straight beams, the location for $\mathrm{R}$ can be chosen such that transverse loads applied at $\mathrm{R}$ will not produce torsion. This location for $\mathrm{R}$ is referred to as the principal pole, or more commonly, the shear center.

As previously stated, the Kirchhoff-Love assumption is assumed to govern the deformation for thin shell elements of the curved beam. This implies that the transverse shear strains $\gamma_{\theta \zeta}$ and $\gamma_{s \zeta}$ vanish. Shear strain $\gamma_{s \zeta}$ vanishes on the basis of Eqs.(2.23) and (2.26). Substituting Eq.(2.42) into Eq.(2.40) and setting the coefficient of $\tau$ to zero (i.e. $\gamma_{\theta z}=0$ ) results in

$$
-\left(a-z_{R}\right) q_{R}-(a-z) \frac{\partial}{\partial \zeta}\left(\frac{\omega}{a-z}\right)=0
$$

The radius expression (a-z) appears frequently, and may be rewritten using Eq.(2.4) as

$$
(a-z)=(a-\bar{z})\left(1+\zeta / R_{\theta}\right)
$$

in which the term $1 / R_{\theta}$ represents the normal curvature of the $\theta$-curve in the shell element reference surface given by

$$
\frac{1}{\mathbf{R}_{\theta}}=\frac{\cos \alpha}{(a-\bar{z})}
$$

Substituting Eq.(2.47)for (a-z) in Eq.(2.46), and rearranging the resulting expression, gives

$$
\frac{\partial}{\partial \zeta}\left(\frac{\omega}{1+\zeta / R_{\theta}}\right)=-\left[\frac{a-z_{R}}{a-\bar{z}}\right] \frac{q_{R}}{\left(1+\zeta / R_{\theta}\right)^{2}}
$$

Integrating this equation with respect to $\zeta$ results in 


$$
\omega=\left[\frac{a-z_{R}}{a-\bar{z}}\right] R_{\theta} q_{R}+\left(1+\zeta / R_{\theta}\right) f(s)
$$

in which $\mathrm{f}(\mathrm{s})$ is an unknown function. Since $\omega=\bar{\omega}$ for $\zeta=0$, function $\mathrm{f}(\mathrm{s})$ can be determined as

$$
f(s)=-\left[\frac{a-z_{R}}{a-\bar{z}}\right] R_{\theta} q_{R}+\bar{\omega}
$$

Upon substitution of $f(s)$ back into the expression for $\omega$, the final expression for the warping function is

$$
\omega(\zeta, s)=\bar{\omega}(s)+\zeta \tilde{\omega}(s)
$$

in which the thickness warping is

$$
\tilde{\omega}(s)=-\left[\frac{a-z_{R}}{a-\bar{z}}\right] q_{R}+\frac{\bar{\omega}}{R_{\theta}}
$$

The thickness warping term can be discontinuous at branch junctions, and its contribution may be questionable. This component of warping is neglected in some thin-walled beam theories. Differing values of $\tilde{\omega}$ from shell branches meeting at a junction implies incompatible circumferential displacements off the contour line. Recall that $\bar{\omega}$ is selected such that the circumferential displacement is continuous on the contour at the junctions. Gjels$\mathrm{vik}^{[46]}$ shows that $\tilde{\omega}$ can be the primary contribution to the total warping $\omega$ for some analysis cases. The example he presents is a cross section composed of a thin straight strip. For this example, $\bar{\omega}$ equals zero since $r(s)=0$ for a reference point on the contour line. Recall 
that $r(s)$ is the perpendicular coordinate from the reference point to the contour line.

The dependence of the shear strain $\gamma_{\theta s}$ on $\zeta$ is obtained from Eq.(2.39) as follows.

Eq.(2.47) is used to replace the radius term (a-z). The arc length of a curve parallel to the contour is $\left(1+\zeta / R_{s}\right)$ ds, where ds is the arc length of the contour. Eq.(2.39) is multiplied by $\left(1+\zeta / R_{s}\right)$ to account for the length of the curve parallel to the contour, and Eq.(2.51) is used to get

$$
\begin{aligned}
(a-\bar{z})\left(1+\zeta / R_{s}\right) & \left(1+\zeta / R_{\theta}\right) \gamma_{\theta s}= \\
& \left(\left(a-z_{R}\right)\left(1+\zeta / R_{s}\right)\left(r_{R}+\zeta\right)-(a-z) \frac{\partial}{\partial s}\left(\frac{\omega}{a-z}\right)\right)_{\tau}
\end{aligned}
$$

Expand the derivative term in Eq.(2.52) using Eq.(2.5), and (2.50), and also use the expression (2.51) for the warping as an explicit function of $\zeta$, to obtain

$$
\begin{aligned}
(\mathrm{a}-\overline{\mathrm{z}})( & \left.1+\zeta / \mathrm{R}_{\mathrm{s}}\right)\left(1+\zeta / \mathrm{R}_{\theta}\right) \gamma_{\theta \mathrm{s}}= \\
& \left(\left[\left(\mathrm{a}-\mathrm{z}_{\mathrm{R}}\right) \mathrm{r}_{\mathrm{R}}-(\mathrm{a}-\overline{\mathrm{z}}) \frac{\mathrm{d} \bar{\omega}}{\mathrm{ds}}-\bar{\omega} \sin \alpha\right]\right. \\
& +\zeta\left[\left(\mathrm{a}-\mathrm{z}_{\mathrm{R}}\right)\left(1+\mathrm{r}_{\mathrm{R}} / \mathrm{R}_{\mathrm{s}}\right)-(\mathrm{a}-\overline{\mathrm{z}})\left(\frac{1}{\mathrm{R}_{\theta}} \frac{\mathrm{d} \bar{\omega}}{\mathrm{ds}}+\frac{\mathrm{d} \tilde{\omega}}{\mathrm{ds}}\right)-(\bar{\omega}\right. \\
& \left.\left.\left(\overline{\mathrm{R}_{\mathrm{s}}}\right) \tilde{\omega}\right) \sin \alpha\right] \\
& \left.+\zeta^{2}\left[\frac{\left(\mathrm{a}-\mathrm{z}_{\mathrm{R}}\right)}{\mathrm{R}_{\mathrm{s}}}-(\mathrm{a}-\overline{\mathrm{z}}) \frac{1}{\mathrm{R}_{\theta}} \frac{\mathrm{d} \tilde{\omega}}{\mathrm{ds}}-\frac{\tilde{\omega}}{\mathrm{R}_{\mathrm{s}}} \sin \alpha\right]\right) \tau
\end{aligned}
$$

The first term on the right-hand side of this equation is zero by the Vlasov assumption. This can be verified by expanding the derivative in Eq. (2.43). Using Eqs.(2.2), (2.9), (2.14), (2.43), (2.48), (2.50), and (2.51), the terms that are linear and quadratic in $\zeta$ on the right hand side of Eq.(2.53) simplify. After simplification, the in-plane shear strain is 


$$
\gamma_{\theta s}=\frac{\zeta\left[1+\frac{\zeta}{2}\left(\frac{1}{R_{s}}+\frac{1}{R_{\theta}}\right)\right]}{\left(1+\zeta / R_{s}\right)\left(1+\zeta / R_{\theta}\right)}(2)\left(\frac{a-z_{R}}{a-\bar{z}}\right)^{2} \tau
$$

The nonzero strains referenced to shell coordinates are $\varepsilon_{\theta \theta}$ in Eq.(2.22) and $\gamma_{\theta s}$ in Eq. (2.54). Using Eq (2.47), these strains can be rewritten as

$$
\begin{aligned}
&\left(\frac{a-\bar{z}}{a-z_{R}}\right)\left(1+\zeta / R_{\theta}\right) \varepsilon_{\theta \theta}= \\
& e_{R}-\left(y-y_{R}\right) \kappa_{z R}+\left(z-z_{R}\right) \kappa_{y R}-\omega\left(\tau /\left(a-z_{R}\right)\right) \\
&\left(\frac{a-\bar{z}}{a-z_{R}}\right)\left(1+\zeta / R_{\theta}\right)\left(1+\zeta / R_{s}\right) \gamma_{\theta s}= \\
& 2\left(\frac{a-z_{R}}{a-\bar{z}}\right) \zeta\left[1+\frac{\zeta}{2}\left(\frac{1}{R_{s}}+\frac{1}{R_{\theta}}\right)\right] \tau
\end{aligned}
$$

in which the bar strains are defined as

$$
\begin{aligned}
& \mathrm{e}_{\mathrm{R}}=\frac{\mathrm{U}_{\mathrm{R}}^{\prime}-\mathrm{W}_{\mathrm{R}}}{\mathrm{a}-\mathrm{z}_{\mathrm{R}}} \\
& \boldsymbol{\kappa}_{\mathrm{zR}}=\frac{\Phi_{\mathrm{zR}}^{\prime}+\Phi_{\mathrm{xR}}}{\mathrm{a}-\mathrm{z}_{\mathrm{R}}} \\
& \kappa_{\mathrm{yR}}=\frac{\Phi_{y R}^{\prime}}{a-z_{\mathrm{R}}}
\end{aligned}
$$

The quantity $e_{R}$ represents the circumferential stretching strain of the arc passing through the point R. $\kappa_{2 \mathrm{R}}$ is the change in curvature out of the plane of the curved beam, and $\kappa_{\mathrm{yR}}$ is the change in curvature in the plane of the curved beam. Substituting the relation for $y$ given by Eq. (2.3), and the relation for $z$ given by Eq. (2.4), and the relation for $\omega$ given by Eq.(2.50), into Eq. (2.55) gives the circumferential strain as an explicit function of $\zeta$. 


$$
\begin{aligned}
\left(\frac{a-\bar{z}}{a-z_{R}}\right)\left(1+\zeta / R_{\theta}\right) & \varepsilon_{\theta \theta}= \\
& e_{\mathrm{R}}-\left(\bar{y}-y_{R}\right) \kappa_{z R}+\left(\bar{z}-z_{R}\right) \kappa_{y R}-\bar{\omega}\left(\tau^{\prime} /\left(a-z_{R}\right)\right) \\
& -\zeta\left(\sin (\alpha) \kappa_{z R}+\cos (\alpha) \kappa_{y R}+\tilde{\omega}\left(\tau^{\prime} /\left(a-z_{R}\right)\right)\right)
\end{aligned}
$$

\subsection{STRAIN ENERGY}

The variation of strain energy, or internal virtual work, for the curved beam can now be expressed in terms of the nonzero virtual strains as

$$
\delta \overline{\mathrm{U}}=\int_{V_{0 l}}\left(\sigma_{\theta \theta} \delta \varepsilon_{\theta \theta}+\sigma_{\theta s} \delta \gamma_{\theta s}\right) \mathrm{dVol}
$$

where $\sigma_{\theta \theta}$ is the circumferential normal stress, and $\sigma_{\theta s}$ is the shear stress. The differential volume element is

$$
d \text { Vol }=(a-z) d \theta d A
$$

in which $\mathrm{dA}$ is the differential area element in the cross section. Using Eq.(2.47), and expressing the differential area element within the cross-section in terms of shell coordinates $\zeta$, and $s,\left(d A=\left(1+\zeta / R_{s}\right) d \zeta d s\right)$, the differential volume element is

$$
\mathrm{dVol}=\frac{(\mathrm{a}-\overline{\mathrm{z}})}{\left(\mathrm{a}-\mathrm{z}_{\mathrm{R}}\right)}\left(1+\zeta / \mathrm{R}_{\theta}\right)\left(1+\zeta / \mathrm{R}_{\mathrm{s}}\right) \mathrm{d} \zeta \mathrm{ds}\left(\mathrm{a}-\mathrm{z}_{\mathrm{R}}\right) \mathrm{d} \theta
$$

The virtual shell strains $\delta \varepsilon_{\theta \theta}$ and $\delta \gamma_{\theta s}$ are obtained in terms of the virtual bar strains $\delta \mathrm{e}_{\mathrm{R}}$, $\delta \kappa_{z R}, \delta \kappa_{y R}, \delta \tau^{\prime}$ and $\delta \tau$, using Eqs. (2.56), and (2.60). These virtual bar strains are substituted into the strain energy variation (2.61) using the differential volume element given by Eq. (2.62). Integrations over the cross section are performed, and the resulting strain 
energy variation for the curved beam is written as

$$
\begin{aligned}
\delta \bar{U} & =\int_{\theta_{1}}^{\theta_{2}}\left[N_{R} \delta e_{R}+M_{Z R} \delta \kappa_{Z R}+M_{Y R} \delta \kappa_{Y R}\right. \\
& \left.+M_{\omega} \delta\left(\tau^{\prime} /\left(a-z_{R}\right)\right)+T_{S R} \delta \tau\right]\left(a-z_{R}\right) d \theta
\end{aligned}
$$

where $\theta_{2}>\theta_{1}$, and the bar resultants $N_{R}, M_{Z R}, M_{Y R}, M_{\omega b}$ and $T_{S R}$ are defined in terms of stresses $\sigma_{\theta \theta}$ and $\sigma_{\theta S}$ in two steps. First, the shell resultants $N_{\theta}, M_{\theta}$, and $M_{\theta S}$ are defined by the thickness integrals

$$
\begin{aligned}
& \left(\mathrm{N}_{\theta}, \mathrm{M}_{\theta}\right)=\int_{\mathrm{h}}(1, \zeta) \sigma_{\theta \theta}\left(1+\zeta / \mathrm{R}_{\mathrm{S}}\right) \mathrm{d} \zeta \\
& \mathrm{M}_{\theta \mathrm{S}}=\int_{\mathrm{h}} \sigma_{\theta \mathrm{S}} \zeta\left[1+\zeta \frac{1}{2}\left(\frac{1}{\mathrm{R}_{\mathrm{S}}}+\frac{1}{\mathrm{R}_{\theta}}\right)\right] \mathrm{d} \zeta
\end{aligned}
$$

Second, the bar resultants are defined by contour integrals of the shell resultants by

$$
\begin{aligned}
& N_{R}=\int_{c} N_{\theta} d s \\
& M_{Z R}=-\int_{c}\left[\left(\bar{y}-y_{R}\right) N_{\theta}+M_{\theta} \sin (\alpha)\right] d s \\
& M_{Y R}=\int_{c}\left[\left(\bar{z}-z_{R}\right) N_{\theta}-M_{\theta} \cos (\alpha)\right] d s \\
& M_{\omega R}=-\int_{c}\left(N_{\theta} \bar{\omega}+M_{\theta} \tilde{\omega}\right) d s \\
& T_{S R}=2 \int_{c} \frac{\left(a-z_{R}\right)}{(a-\bar{z})} M_{\theta S} d s
\end{aligned}
$$


in which $N_{R}$ is the circumferential force at the reference point $R, M_{Y R}$, and $M_{Z R}$ are bending moments about the $y$ - and $z$-axis at $R, M_{\omega R}$ is the bimoment, and $T_{S R}$ is the Saint Venant torque. These resultants are shown in Figure 2.10. A triple headed arror is used to depict the bimoment since it is neither a force or moment, and has dimension units of force times length squared. Note that the resultant $\mathbf{M}_{\theta \mathrm{s}}$ defined by Eq.(2.65) is a mathematical quantity, and is not the same as the shell twisting moment defined as $\int \sigma_{\theta S} \zeta\left(1+\zeta / R_{S}\right) d \zeta$

h

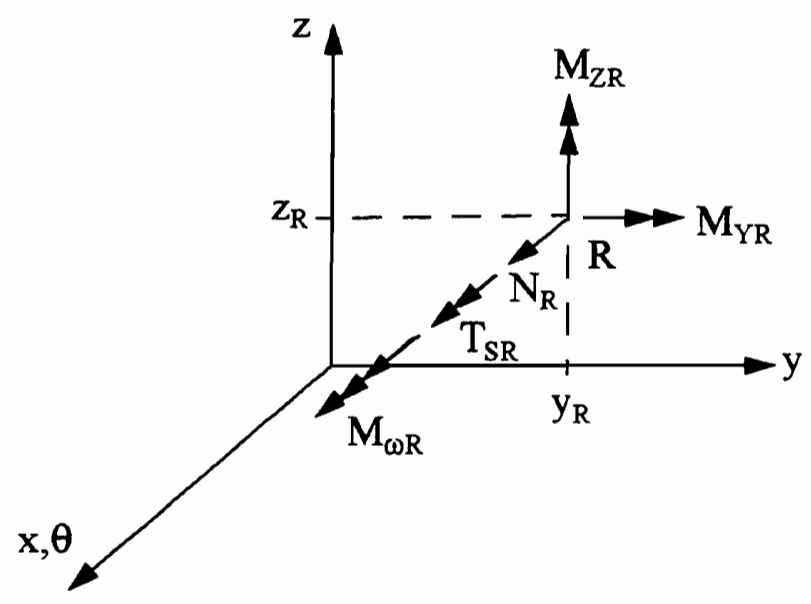

Figure 2.10 Positive bar resultants

\subsection{HOOKE'S LAW}

For a laminated composite material in the s- $\theta$ plane, a plane stress state is defined by assuming that the thickness normal stress $\sigma_{\zeta \zeta}$, and the thickness shear stresses $\sigma_{\mathrm{S} \zeta}$, and $\sigma_{\zeta \theta}$ are zero. For this assumption, the stress-strain law for a lamina oriented at an arbitrary angle in the $s-\theta$ plane is 


$$
\left\{\begin{array}{c}
\sigma_{\mathrm{SS}} \\
\sigma_{\theta \theta} \\
\sigma_{\mathrm{S} \theta}
\end{array}\right\}=\left[\begin{array}{lll}
\overline{\mathrm{Q}}_{11} & \overline{\mathrm{Q}}_{12} & \overline{\mathrm{Q}}_{16} \\
\overline{\mathrm{Q}}_{12} & \overline{\mathrm{Q}}_{22} & \overline{\mathrm{Q}}_{26} \\
\overline{\mathrm{Q}}_{16} & \overline{\mathrm{Q}}_{26} & \overline{\mathrm{Q}}_{66}
\end{array}\right]\left\{\begin{array}{c}
\varepsilon_{\mathrm{SS}} \\
\varepsilon_{\theta \theta} \\
\gamma_{\mathrm{S} \theta}
\end{array}\right\}
$$

in which $\overline{\mathrm{Q}}_{\mathrm{ij}}, \mathrm{i}=1,2,6$, are the transformed reduced stiffnesses. The reduced stiffnesses $\mathrm{Q}_{\mathrm{ij}}$ are calculated from material properties in the principle material direction and transformed to $\overline{\mathrm{Q}}_{\mathrm{ij}}$ at the specified angle for each lamina.

The distribution of the strains in the thickness coordinate $\zeta$ follow from the KirchhoffLove assumption in shell theory. For Sander's shell theory ${ }^{51}{ }^{3}$, these strain distributions are

$$
\begin{aligned}
\varepsilon_{\mathrm{SS}}= & \frac{\varepsilon_{\mathrm{SS}}^{\mathrm{o}}+\zeta \kappa_{\mathrm{SS}}^{\mathrm{o}}}{1+\zeta / \mathrm{R}_{\mathrm{S}}} \\
\varepsilon_{\theta \theta}= & \frac{\varepsilon_{\theta \theta}^{\mathrm{o}}+\zeta \kappa_{\theta \theta}^{\mathrm{o}}}{1+\zeta / \mathrm{R}_{\theta}} \\
\gamma_{\mathrm{S} \theta}= & \left\{\left[1+\frac{\zeta}{2}\left(\frac{1}{\mathrm{R}_{\theta}}+\frac{1}{\mathrm{R}_{\mathrm{S}}}\right)+\frac{\zeta^{2}}{4}\left(\frac{1}{\mathrm{R}_{\theta}}-\frac{1}{\mathrm{R}_{\mathrm{S}}}\right)^{2}\right] \gamma_{\mathrm{S} \theta}^{\mathrm{O}}\right. \\
& \left.+\zeta\left[1+\frac{\zeta}{2}\left(\frac{1}{\mathrm{R}_{\theta}}+\frac{1}{\mathrm{R}_{\mathrm{S}}}\right)\right] \mathrm{K}_{\mathrm{S} \theta}^{\mathrm{o}}\right\} /\left[\left(1+\zeta / \mathrm{R}_{\mathrm{S}}\right)\left(1+\zeta / \mathrm{R}_{\theta}\right)\right]
\end{aligned}
$$

in which $\varepsilon_{\mathrm{Ss}}^{\mathrm{O}}, \mathrm{\kappa}_{\mathrm{ss}}^{\mathrm{O}}, \varepsilon_{\theta \theta}^{\mathrm{o}}, \mathrm{\kappa}_{\theta \theta}^{\mathrm{o}}, \gamma_{\mathrm{s} \theta}^{\mathrm{o}}$, and $\mathrm{K}_{\mathrm{S} \theta}^{\mathrm{o}}$ are reference surface strain measures that are independent of $\zeta$. The shell resultants in Sander's theory are

$$
\left(\mathrm{N}_{\mathrm{S}}, \mathrm{M}_{\mathrm{S}}\right)=\int_{\mathrm{h}}(1, \zeta) \sigma_{\mathrm{SS}}\left(1+\zeta / \mathrm{R}_{\theta}\right) \mathrm{d} \zeta
$$




$$
\begin{aligned}
& \left(\mathrm{N}_{\theta}, \mathrm{M}_{\theta}\right)=\int_{\mathrm{h}}(1, \zeta) \sigma_{\theta \theta}\left(1+\zeta / \mathrm{R}_{\mathrm{S}}\right) \mathrm{d} \zeta \\
& \mathrm{N}_{\mathrm{S} \theta}=\mathrm{N}_{\theta \mathrm{S}}=\int_{\mathrm{h}} \sigma_{\mathrm{S} \theta}\left[1+\frac{\zeta}{2}\left(\frac{1}{\mathrm{R}_{\theta}}+\frac{1}{\mathrm{R}_{\mathrm{S}}}\right)+\frac{\zeta^{2}}{4}\left(\frac{1}{\mathrm{R}_{\theta}}-\frac{1}{\mathrm{R}_{\mathrm{S}}}\right)^{2}\right] \mathrm{d} \zeta \\
& \mathrm{M}_{\mathrm{S} \theta}=\mathrm{M}_{\theta \mathrm{S}}=\int_{\mathrm{h}} \sigma_{\mathrm{S} \theta} \zeta\left[1+\frac{\zeta}{2}\left(\frac{1}{\mathrm{R}_{\theta}}+\frac{1}{\mathrm{R}_{\mathrm{S}}}\right)\right] \mathrm{d} \zeta
\end{aligned}
$$

Again, note that $\mathrm{N}_{\mathrm{S} \theta}$ and $\mathrm{M}_{\mathrm{S} \theta}$ resultants are mathematical definitions and not physical quantities.

The expressions for shell resultants given by Sander's theory are consistent with those previously derived as Eqs.(2.64) and (2.65). Substituting Eqs.(2.71) to (2.74) into these shell resultants, the stress-strain law for shell variables is

$$
\left\{\begin{array}{c}
\mathrm{N}_{\theta} \\
\mathrm{N}_{\mathrm{S}} \\
\mathrm{N}_{\theta \mathrm{S}} \\
\mathbf{M}_{\theta} \\
\mathbf{M}_{\mathrm{S}} \\
\mathbf{M}_{\theta \mathrm{S}}
\end{array}\right\}=\left[\begin{array}{llllll}
\mathrm{A}_{11} & \mathrm{~A}_{12} & \mathrm{~A}_{16} & \mathrm{~B}_{11} & \mathrm{~B}_{12} & \mathrm{~B}_{16} \\
\mathrm{~A}_{12} & \mathrm{~A}_{22} & \mathrm{~A}_{26} & \mathrm{~B}_{12} & \mathrm{~B}_{22} & \mathrm{~B}_{26} \\
\mathrm{~A}_{16} & \mathrm{~A}_{26} & \mathrm{~A}_{66} & \mathrm{~B}_{61} & \mathrm{~B}_{62} & \mathrm{~B}_{66} \\
\mathrm{~B}_{11} & \mathrm{~B}_{12} & \mathrm{~B}_{61} & \mathrm{D}_{11} & \mathrm{D}_{12} & \mathrm{D}_{16} \\
\mathrm{~B}_{12} & \mathrm{~B}_{22} & \mathrm{~B}_{62} & \mathrm{D}_{12} & \mathrm{D}_{22} & \mathrm{D}_{26} \\
\mathrm{~B}_{16} & \mathrm{~B}_{26} & \mathrm{~B}_{66} & \mathrm{D}_{16} & \mathrm{D}_{26} & \mathrm{D}_{66}
\end{array}\right]\left\{\begin{array}{c}
\varepsilon_{\theta \theta}^{\mathrm{o}} \\
\varepsilon_{\mathrm{SS}}^{\mathrm{o}} \\
\gamma_{\theta \mathrm{S}}^{\mathrm{o}} \\
\kappa_{\theta \theta}^{\mathrm{o}} \\
\kappa_{\mathrm{SS}}^{\mathrm{o}} \\
\kappa_{\theta \mathrm{S}}^{\mathrm{o}}
\end{array}\right\}
$$

in which the stiffness terms are

$$
\begin{aligned}
& \left(\mathrm{A}_{11}, \mathrm{~B}_{11}, \mathrm{D}_{11}\right)=\int_{\mathrm{h}}\left(1, \zeta, \zeta^{2}\right) \overline{\mathrm{Q}}_{11} \frac{\left(1+\zeta / \mathrm{R}_{\theta}\right)}{\left(1+\zeta / \mathrm{R}_{\mathrm{s}}\right)} \mathrm{d} \zeta \\
& \left(\mathrm{A}_{12}, \mathrm{~B}_{12}, \mathrm{D}_{12}\right)=\int_{\mathrm{h}}\left(1, \zeta, \zeta^{2}\right) \overline{\mathrm{Q}}_{12} \mathrm{~d} \zeta
\end{aligned}
$$




$$
\begin{aligned}
& \left(\mathrm{A}_{22}, \mathrm{~B}_{22}, \mathrm{D}_{22}\right)=\int_{\mathrm{b}}\left(1, \zeta, \zeta^{2}\right) \overline{\mathrm{Q}}_{22} \frac{\left(1+\zeta / \mathrm{R}_{\mathrm{s}}\right)}{\left(1+\zeta / \mathrm{R}_{\theta}\right)} \mathrm{d} \zeta \\
& \mathrm{A}_{66}=\int_{\mathrm{b}} \overline{\mathrm{Q}}_{66} \frac{\left[1+\frac{\zeta}{2}\left(\frac{1}{\mathrm{R}_{\theta}}+\frac{1}{\mathrm{R}_{\mathrm{S}}}\right)+\frac{\zeta^{2}}{4}\left(\frac{1}{\mathrm{R}_{\theta}}-\frac{1}{\mathrm{R}_{\mathrm{S}}}\right)^{2}\right]^{2}}{\left(1+\zeta / \mathrm{R}_{\mathrm{s}}\right)\left(1+\zeta / \mathrm{R}_{\theta}\right)} \mathrm{d} \zeta \\
& \mathrm{B}_{66}=\int_{\mathrm{h}} \overline{\mathrm{Q}}_{66} \frac{\zeta\left[1+\frac{\zeta}{2}\left(\frac{1}{\mathrm{R}_{\theta}}+\frac{1}{\mathrm{R}_{\mathrm{S}}}\right)\right]\left[1+\frac{\zeta}{2}\left(\frac{1}{\mathrm{R}_{\theta}}+\frac{1}{\mathrm{R}_{\mathrm{S}}}\right)+\frac{\zeta^{2}}{4}\left(\frac{1}{\mathrm{R}_{\theta}}-\frac{1}{\mathrm{R}_{\mathrm{S}}}\right)^{2}\right]}{\left(1+\zeta / \mathrm{R}_{\mathrm{s}}\right)\left(1+\zeta / \mathrm{R}_{\theta}\right)} \mathrm{d} \zeta \\
& \mathrm{D}_{66}=\int_{\mathrm{h}} \overline{\mathrm{Q}}_{66} \frac{\zeta^{2}\left[1+\frac{\zeta}{2}\left(\frac{1}{\mathrm{R}_{\theta}}+\frac{1}{\mathrm{R}_{\mathrm{S}}}\right)\right]^{2}}{\left(1+\zeta / \mathrm{R}_{\mathrm{s}}\right)\left(1+\zeta / \mathrm{R}_{\theta}\right)} \mathrm{d} \zeta \\
& \mathrm{A}_{16}=\int_{\mathrm{h}} \overline{\mathrm{Q}}_{16} \frac{1+\frac{\zeta}{2}\left(\frac{1}{\mathrm{R}_{\theta}}+\frac{1}{\mathrm{R}_{\mathrm{S}}}\right)+\frac{\zeta^{2}}{4}}{\left(1+\zeta / \mathrm{R}_{\mathrm{s}}\right)} \mathrm{d} \zeta \\
& \mathrm{B}_{16}=\int_{\mathrm{h}} \overline{\mathrm{Q}}_{16} \zeta\left[\frac{1+\frac{\zeta}{2}\left(\frac{1}{\mathrm{R}_{\theta}}+\frac{1}{\mathrm{R}_{\mathrm{S}}}\right)}{\left(1+\zeta / \mathrm{R}_{\mathrm{s}}\right)}\right] \mathrm{d} \zeta \\
& \mathrm{A}_{26}=\int_{\mathrm{h}} \overline{\mathrm{Q}}_{26} \frac{1+\frac{\zeta}{2}\left(\frac{1}{\mathrm{R}_{\theta}}+\frac{1}{\mathrm{R}_{\mathrm{S}}}\right)+\frac{\zeta^{2}}{4}\left(\frac{1}{\mathrm{R}_{\theta}}-\frac{1}{\mathrm{R}_{\mathrm{S}}}\right)^{2}}{\left(1+\zeta / \mathrm{R}_{\theta}\right)} \mathrm{d} \zeta \\
& \mathrm{B}_{26}=\int_{\mathrm{h}} \overline{\mathrm{Q}}_{26} \zeta\left[\frac{1+\frac{\zeta}{2}\left(\frac{1}{\mathrm{R}_{\theta}}+\frac{1}{\mathrm{R}_{\mathrm{S}}}\right)}{\left(1+\zeta / \mathrm{R}_{\theta}\right)}\right] \mathrm{d} \zeta \\
& \mathbf{B}_{61}=\int_{\mathrm{h}} \overline{\mathrm{Q}}_{16} \frac{\zeta\left[1+\frac{\zeta}{2}\left(\frac{1}{\mathrm{R}_{\theta}}+\frac{1}{\mathrm{R}_{\mathrm{S}}}\right)+\frac{\zeta^{2}}{4}\left(\frac{1}{\mathrm{R}_{\theta}}-\frac{1}{\mathrm{R}_{\mathrm{S}}}\right)^{2}\right]}{\left(1+\zeta / \mathrm{R}_{\mathrm{s}}\right)} \mathrm{d} \zeta
\end{aligned}
$$




$$
\begin{aligned}
& \mathrm{B}_{62}=\int_{\mathrm{h}} \overline{\mathrm{Q}}_{26} \frac{\zeta\left[1+\frac{\zeta}{2}\left(\frac{1}{\mathrm{R}_{\theta}}+\frac{1}{\mathrm{R}_{\mathrm{S}}}\right)+\frac{\zeta^{2}}{4}\left(\frac{1}{\mathrm{R}_{\theta}}-\frac{1}{\mathrm{R}_{\mathrm{S}}}\right)^{2}\right]}{\left(1+\zeta / \mathrm{R}_{\theta}\right)} \mathrm{d} \zeta \\
& \mathrm{D}_{16}=\int_{\mathrm{h}} \overline{\mathrm{Q}}_{16} \zeta^{2}\left[\frac{1+\frac{\zeta}{2}\left(\frac{1}{\mathrm{R}_{\theta}}+\frac{1}{\mathrm{R}_{\mathrm{S}}}\right)}{\left(1+\zeta / \mathrm{R}_{\mathrm{s}}\right)}\right] \mathrm{d} \zeta \\
& \mathrm{D}_{26}=\int_{\mathrm{h}} \overline{\mathrm{Q}}_{26} \zeta^{2}\left[\frac{\left.1+\frac{\zeta\left(\frac{1}{2}\left(\frac{1}{\mathrm{R}_{\theta}}+\frac{1}{\mathrm{R}_{\mathrm{S}}}\right)\right.}{\left(1+\zeta / \mathrm{R}_{\mathrm{s}}\right)}\right] \mathrm{d} \zeta}{\left(1+\zeta{ }^{2}\right.}\right.
\end{aligned}
$$

Under the assumption that the cross section is rigid in its own plane, the strains $\varepsilon_{\mathrm{sS}}^{\mathrm{o}}$, and $\kappa_{\text {ss }}^{\mathrm{O}}$ vanish (see also Eqs (2.23) and (2.26)). From Eqs. (2.56) and (2.60), the shell strain measures in Eqs.(2.73), and (2.74) are identified as

$$
\begin{aligned}
& \varepsilon_{\theta \theta}^{0}=\left(\frac{a-z_{R}}{a-\bar{z}}\right)\left[e_{R}-\left(\bar{y}-y_{R}\right) \kappa_{Z R}+\left(\bar{z}-z_{R}\right) \kappa_{Y R}-\bar{\omega}\left(\frac{\tau^{\prime}}{a-z_{R}}\right)\right] \\
& \kappa_{\theta \theta}^{0}=-\left(\frac{a-z_{R}}{a-\bar{z}}\right)\left[\sin (\alpha) \kappa_{Z R}+\cos (\alpha) \kappa_{Y R}+\tilde{\omega}\left(\frac{\tau^{\prime}}{a-z_{R}}\right)\right] \\
& \gamma_{S \theta}^{o}=0 \\
& \kappa_{S \theta}^{o}=2\left(\frac{a-z_{R}}{a-\bar{z}}\right)^{2} \tau
\end{aligned}
$$

In terms of thin-walled beam theory, the shell resultants which appear on the left hand side of Eq. (2.79) can be classified as being either active or reactive. The active resultants are those resultants directly associated with a deformation (nonvanishing conjugate strain components). Only the active resultants contribute to internal work. Reactive resultants have no associated deformation, but are needed to maintain equilibrium. The reactive shell 
resultants can be determined from the equilibrium equations, and the assumptions used in the derivation of thin-walled beam theory. For thin-walled beam theory, the active and reactive resultants and strains are listed in Table 2.1 (Reactive strains are zero)

Table 2.1: Active and reactive resultants and strains

\begin{tabular}{lll}
\hline \hline & resultants & strains \\
\hline active & $\mathrm{N}_{\theta}, \mathrm{M}_{\theta}, \mathrm{M}_{\theta \mathrm{s}}$ & $\varepsilon_{\theta \theta}^{0}, \kappa_{\theta \theta}^{0}, \kappa_{\mathrm{s} \theta}^{0}$ \\
reactive & $\mathrm{N}_{\mathrm{s}}, \mathrm{N}_{\theta \mathrm{s}}, \mathrm{M}_{\mathrm{s}}$ & $\varepsilon_{\mathrm{ss}}^{\mathrm{o}}, \gamma_{\mathrm{s} \theta}^{\mathrm{o}}, \mathrm{\kappa}_{\mathrm{s} s}^{\mathrm{O}}$ \\
\hline \hline
\end{tabular}

The rows and columns of Eq.(2.79) are rearranged, separating the active resultants from the reactive resultants

$$
\left\{\begin{array}{c}
N_{\theta} \\
M_{\theta} \\
M_{\theta S} \\
N_{S} \\
N_{\theta S} \\
M_{S}
\end{array}\right\}=\left[\begin{array}{llllll}
A_{11} & B_{11} & B_{16} & A_{12} & A_{16} & B_{12} \\
B_{11} & D_{11} & D_{16} & B_{12} & B_{61} & D_{12} \\
B_{16} & D_{16} & D_{66} & B_{26} & B_{66} & D_{26} \\
A_{12} & B_{12} & B_{26} & A_{22} & A_{26} & B_{22} \\
A_{16} & B_{61} & B_{66} & A_{26} & A_{66} & B_{62} \\
B_{12} & D_{12} & D_{26} & B_{22} & B_{62} & D_{22}
\end{array}\right]\left\{\begin{array}{c}
\varepsilon_{\theta \theta}^{o} \\
\kappa_{\theta \theta}^{o} \\
\kappa_{\theta S}^{o} \\
\varepsilon_{S S}^{o} \\
\gamma_{\theta S}^{o} \\
\kappa_{S S}^{o}
\end{array}\right\}
$$

At this point there are two ways to proceed from Eq. (2.98). Following the assumptions on the deformation one could set $\varepsilon_{\mathrm{SS}}^{0}=\gamma_{\theta \mathrm{S}}^{0}=\kappa_{\mathrm{SS}}^{\mathrm{o}}=0$ in Eq. (2.98) and write the active resultants in terms of the active strains. Alternatively, one could follow the procedure adopted in the Bernoulli-Euler beam theory in which the lateral normal stresses in the beam are neglected relative to the axial normal stress in Hooke's law. The implication of the latter approach for the thin-walled beam theory is to set the reactive resultants to zero 
in Eq.(2.98). These two approaches to Hooke's law formulation, that is either setting the reactive strains to zero or setting the reactive resultants to zero, can be incorporated into the formulation in a convenient manner following the approach used by Wu and Sun ${ }^{52]}$. This procedure is described in the following paragraph.

First, the reactive resultants $\mathrm{N}_{\mathrm{S}}, \mathrm{N}_{\theta \mathrm{S}}$, and $\mathrm{M}_{\mathrm{S}}$ are set to zero. With this assumption, the reactive strains $\varepsilon_{S S}^{0}, \gamma_{S \theta}^{0}$, and $\kappa_{S S}^{0}$ can be solved for in terms of the active strains $\varepsilon_{\theta \theta}^{o}, \kappa_{\theta \theta}^{o}$, and $\kappa_{s \theta}^{o}$ from the last three equations of the matrix Eq. (2.98). The result is

$$
\left\{\begin{array}{c}
\varepsilon_{\mathrm{SS}}^{\mathrm{o}} \\
\gamma_{\theta \mathrm{S}}^{\mathrm{O}} \\
\kappa_{\mathrm{SS}}^{\mathrm{o}}
\end{array}\right\}=-\left[\begin{array}{lll}
\mathrm{A}_{22} & \mathrm{~A}_{26} & \mathrm{~B}_{22} \\
\mathrm{~A}_{26} & \mathrm{~A}_{66} & \mathrm{~B}_{62} \\
\mathrm{~B}_{22} & \mathrm{~B}_{62} & \mathrm{D}_{22}
\end{array}\right]^{-1}\left[\begin{array}{lll}
\mathrm{A}_{12} & \mathrm{~B}_{12} & \mathrm{~B}_{26} \\
\mathrm{~A}_{16} & \mathrm{~B}_{61} & \mathrm{~B}_{66} \\
\mathrm{~B}_{12} & \mathrm{D}_{12} & \mathrm{D}_{26}
\end{array}\right]\left[\begin{array}{c}
\varepsilon_{\theta \theta}^{\mathrm{o}} \\
\kappa_{\theta \theta}^{\mathrm{o}} \\
\kappa_{\theta \mathrm{S}}^{\mathrm{o}}
\end{array}\right\}
$$

Substituting these results into the first three equations of the matrix Eq. (2.98) we get

$$
\left\{\begin{array}{c}
N_{\theta} \\
M_{\theta} \\
M_{\theta S}
\end{array}\right\}=\left[\begin{array}{lll}
a_{11} & b_{11} & b_{16} \\
b_{11} & d_{11} & d_{16} \\
b_{16} & d_{16} & d_{66}
\end{array}\right]\left\{\begin{array}{c}
\varepsilon_{\theta \theta}^{o} \\
\kappa_{\theta \theta}^{o} \\
\kappa_{\theta S}^{o}
\end{array}\right\}
$$

where 


$$
\begin{aligned}
& {\left[\begin{array}{lll}
a_{11} & b_{11} & b_{16} \\
b_{11} & d_{11} & d_{16} \\
b_{16} & d_{16} & d_{66}
\end{array}\right]=} \\
& {\left[\begin{array}{lll}
A_{11} & B_{11} & B_{16} \\
B_{11} & D_{11} & D_{16} \\
B_{16} & D_{16} & D_{66}
\end{array}\right]-(\delta)\left[\begin{array}{lll}
A_{12} & A_{16} & B_{12} \\
B_{12} & B_{61} & D_{12} \\
B_{26} & B_{66} & D_{26}
\end{array}\right]\left[\begin{array}{lll}
A_{22} & A_{26} & B_{22} \\
A_{26} & A_{66} & B_{62} \\
B_{22} & B_{62} & D_{22}
\end{array}\right]^{-1}\left[\begin{array}{llll}
A_{12} & B_{12} & B_{26} \\
A_{16} & B_{61} & B_{66} \\
B_{12} & D_{12} & D_{26}
\end{array}\right]}
\end{aligned}
$$

and dimensionless parameter $\delta$ is a value between zero and one. For $\delta=0$, Hooke's law is based on the reactive strains vanishing, and for $\delta=1$ Hooke's law is based on the reactive resultants vanishing.

Eqs (2.94) - (2.97) are substituted into the right hand side of Eq. (2.100) to obtain the active shell resultants in terms of the bar strains and the laminate stiffness matrix. These expressions for the active shell resultants are then substituted into Eqs. (2.66) - (2.70) to obtain the bar resultants in terms of the bar strains. The result of these manipulations is

$$
\left\{\begin{array}{c}
\mathrm{N}_{\mathrm{R}} \\
\mathrm{M}_{\mathrm{YR}} \\
\mathrm{M}_{\mathrm{ZR}} \\
\mathrm{M}_{\omega \mathrm{R}} \\
\mathrm{T}_{\mathrm{SR}}
\end{array}\right\}=\left[\begin{array}{ccccc}
\mathrm{EA} & \mathrm{ES}_{\mathrm{y}} & -\mathrm{ES}_{\mathrm{z}} & -\mathrm{ES}_{\omega} & \mathrm{EH} \\
\mathrm{ES}_{\mathrm{y}} & \mathrm{EI}_{\mathrm{yy}} & -\mathrm{EI}_{\mathrm{zy}} & -\mathrm{EI}_{\omega \mathrm{y}} & \mathrm{EH}_{\mathrm{c}} \\
-\mathrm{ES}_{\mathrm{z}} & -\mathrm{EI}_{\mathrm{zy}} & \mathrm{EI}_{\mathrm{zz}} & \mathrm{EI}_{\omega \mathrm{z}} & -\mathrm{EH}_{\mathrm{s}} \\
-\mathrm{ES}_{\omega} & -\mathrm{EI}_{\omega \mathrm{y}} & \mathrm{EI}_{\omega \mathrm{z}} & \mathrm{EI}_{\omega \omega} & -\mathrm{EH}_{\mathrm{q}} \\
\mathrm{EH} & \mathrm{EH}_{\mathrm{c}} & -\mathrm{EH}_{\mathrm{s}} & -\mathrm{EH}_{\mathrm{q}} & \mathrm{GJ}
\end{array}\right]\left\{\begin{array}{c}
\varepsilon_{\mathrm{R}} \\
\kappa_{\mathrm{YR}} \\
\kappa_{\mathrm{ZR}} \\
\tau_{\mathrm{R}}^{\prime} /\left(\mathrm{a}-\mathrm{z}_{\mathrm{R}}\right) \\
\tau_{\mathrm{R}}
\end{array}\right\}
$$

in which

$$
\begin{aligned}
& E A=\int_{c} a_{11}\left(\frac{a-z_{R}}{a-\bar{z}}\right) d s \\
& E_{y}=\int_{c}\left(a_{11}\left(\bar{z}-z_{R}\right)-b_{11} \cos \alpha\right)\left(\frac{a-z_{R}}{a-\bar{z}}\right) d s
\end{aligned}
$$




$$
\begin{aligned}
& E S_{z}=\int_{c}\left(a_{11}\left(\bar{y}-y_{R}\right)+b_{11} \sin \alpha\right)\left(\frac{a-z_{R}}{a-\bar{z}}\right) d s \\
& E S_{\omega}=\int_{c}\left(a_{11} \bar{\omega}+b_{11} \tilde{\omega}\right)\left(\frac{a-z_{R}}{a-\bar{z}}\right) d s \\
& E H=2 \int_{c} b_{16}\left(\frac{a-z_{R}}{a-\bar{z}}\right)^{2} d s \\
& E I_{y y}=\int_{c}\left(a_{11}\left(\bar{z}-z_{R}\right)^{2}-2 b_{11}\left(\bar{z}-z_{R}\right) \cos \alpha+d_{11} \cos ^{2} \alpha\right)\left(\frac{a-z_{R}}{a-\bar{z}}\right) d s \\
& E I_{z y}=\int_{c}\left[a_{11}\left(\bar{z}-z_{R}\right)\left(\bar{y}-y_{R}\right)+b_{11}\left(\left(\bar{z}-z_{R}\right) \sin \alpha-\left(\bar{y}-y_{R}\right) \cos \alpha\right)\right. \\
& \left.-d_{11} \sin \alpha \cos \alpha\right]\left(\frac{a-z_{R}}{a-\bar{z}}\right) d s \\
& E I_{\omega y}=\int_{c}\left[a_{11}\left(\bar{z}-z_{R}\right) \bar{\omega}+b_{11}\left(\left(\bar{z}-z_{R}\right) \tilde{\omega}-\bar{\omega} \cos \alpha\right)\right. \\
& \left.-d_{11} \tilde{\omega} \cos \alpha\right]\left(\frac{a-z_{R}}{a-\bar{z}}\right) d s \\
& E H_{c}=2 \int\left(b_{16}\left(\bar{z}-z_{R}\right)-d_{16} \cos \alpha\right)\left(\frac{a-z_{R}}{a-\bar{z}}\right)^{2} d s \\
& E I_{z z}=\int_{c}\left(a_{11}\left(\bar{y}-y_{R}\right)^{2}+2 b_{11}\left(\bar{y}-y_{R}\right) \sin \alpha+d_{11} \sin ^{2} \alpha\right)\left(\frac{a-z_{R}}{a-\bar{z}}\right) d s \\
& E I_{\omega z}=\int_{c}\left[a_{11}\left(\bar{y}-y_{R}\right) \bar{\omega}+b_{11}\left(\left(\bar{y}-y_{R}\right) \tilde{\omega}-\bar{\omega} \sin \alpha\right)\right. \\
& \left.+d_{11} \tilde{\omega} \sin \alpha\right]\left(\frac{a-z_{R}}{a-\bar{z}}\right) d s \\
& E H_{s}=2 \int\left(b_{16}\left(\bar{y}-y_{R}\right)+d_{16} \sin \alpha\right)\left(\frac{a-z_{R}}{a-\bar{z}}\right)^{2} d s
\end{aligned}
$$




$$
\begin{aligned}
& E_{\omega \omega}=\int_{c}\left(a_{11} \bar{\omega}^{-2}+2 b_{11} \bar{\omega} \tilde{\omega}+d_{11} \tilde{\omega}^{2}\right)\left(\frac{a-z_{R}}{a-\bar{z}}\right) d s \\
& E_{q}=\underset{c}{2}\left(b_{16} \bar{\omega}+d_{16} \tilde{\omega}\right)\left(\frac{a-z_{R}}{a-\bar{z}}\right)^{2} d s \\
& G J=4 \int_{c} d_{66}\left(\frac{a-z_{R}}{a-\tilde{z}}\right)^{3} d s
\end{aligned}
$$

The contour integrals of Eqs. (2.103) - (2.117) define the laminated curved beam stiffnesses which appear in Eq. (2.102). These stiffnesses are commonly referred to as modulus weighted-section properties. The "EH" terms are unique to laminated thin-walled beams (see Bauld \& Tzeng[ $\left.{ }^{50}\right]$ ). They represent the stiffness contribution from the coupling terms $b_{16}$, and $d_{16}$ in the laminate stiffness matrix. If the laminate construction for each shell is specially orthotropic, then the "EH" terms are all equal to zero. In the limit as the radius of curvature approaches infinity, these properties reduce to the expressions for straight beams. The expression of Hooke's law for curved laminated thin-walled beams is given by Eq. (2.102). 


\section{CHAPTER 3}

\section{CALCULATION OF CURVED BEAM SECTION PROPERTIES}

\section{1 INTRODUCTION}

For optimization of curved beams, the contour integrals of Eqs.(2.103)-(2.117), must be evaluated for each cross section considered. These calculations are a lengthy, tedious, and error prone task. The objective for the work discussed in this chapter was to develop a general method for evaluating the 15 contour integrals quickly and accurately, using a minimum number of simplifying assumptions. With this in mind, there was considerable motivation to automate the task using a computer program. Although several algorithms are available for straight beams made from isotropic materials ( see Coyette ${ }^{[3]}$ ), no program to calculate the 15 required properties for general curved beam cross sections made from laminated orthotropic materials was known to be available at the time of this writing. This chapter outlines a methodology for calculating the modulus weighted section properties of Eqs (2.103)-(2.117), that was developed as part of this work.

\subsection{EVALUATION OF SECTION CONTOUR INTEGRALS}

When all of the appropriate terms have been substituted into Eqs.(2.103)-(2.117) and the 
products have been expanded, the general difficulty associated with their exact evaluation is appreciated. For the most general case, every term which appears in the contour integrals, with the exception of radius $a$, and reference point coordinates $y_{R}$, and $z_{R}$, can be a function of the shell coordinate $s$. As a result, exact evaluation of the integrals becomes a very large task.

Evaluation of the cross section contour integrals can be simplified considerably by making the following two assumptions.

Cross sections are idealized as an arrangement of connected straight branches.

$$
\left(1 / R_{S}=0\right)
$$

Material properties, stacking sequence, ply orientations, and the shell thickness " $h$ ", remain constant along each cross section branch, and the shell curvature effects in the $r-\theta$ plane can be neglected in the calculation of laminate stiffness properties (i.e. $1 / R_{\theta} \cong 0$, in Eqs.(2.80) through (2.93)).

These two assumptions do not impose practical limitations on the type of cross sections which can be accurately evaluated, and they result in considerable simplification of the contour integrals. Assumption (3.1) requires that curved branches of the cross section be approximated as a series of straight branches. This is accomplished by adding artificial junctions along the curves in the contour. Using straight branches eliminates the dependence of the contour angle $\alpha$ on the shell coordinate $s$. As a result, the transcendental functions in the contour integrals no longer contain the variable of integration. This is a major simplification of the contour integrals since it reduces the transcendental functions to constants along each branch. Although it is not immediately obvious from Eqn.(2.11), the contour coordinate $r_{R}$ is also reduced to a constant for straight contour branches. This feature simplifies the contour warping function $\bar{\omega}$ of Eqn.(2.44), to a linear function of $s$ 
along the branch contour. The thickness warping function $\tilde{\omega}$ of Eqn.(2.51), is also simplified since it is a function of both $\alpha$ and $\bar{\omega}$. Expressions $\bar{\omega}$ and $\tilde{\omega}$ appear individually, and as products in several of the integrals, and the straight branch assumption simplifies those expressions considerably. The next assumption (3.2), insures that the laminate stiffness properties $\mathrm{a}_{\mathrm{ij}}$, $\mathrm{b}_{\mathrm{ij}}$, and $\mathrm{d}_{\mathrm{ij}}$, given by Eqn.(2.101), are constants for each straight branch. Consequently, these terms can be brought outside the contour integrals, and calculated in advance using classical lamination theory. Changes in material properties and shell thicknesses can be approximated using discrete changes from branch to branch. These approximations can be refined by adding additional junctions, and dividing branches, until the desired accuracy is achieved. In practice, these approximate measures are not required, since most practical cross sections actually do consist of straight branches, and material or thickness changes along a branch are normally discrete. The assumption that shell curvature can be neglected in the Sanders shell stiffness equations was tested by implementing Eqs. (2.80) through (2.93) both with, and without the curvature term present. When the ratio of laminate thickness to beam radius is very small, the effect of laminate curvature on beam stiffness is negligible. For most situations, assumptions (3.1), and (3.2), have little effect on the accuracy of the section property calculations.

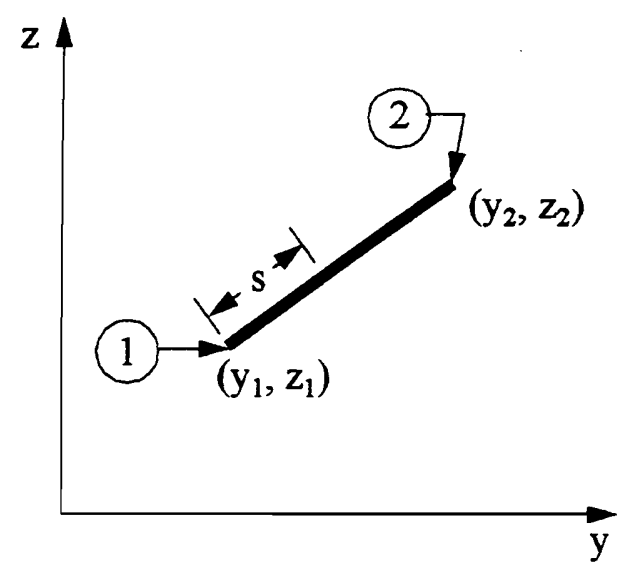

Figure 3.1 Straight branch contour element 
Consider a straight branch between cross-sectional coordinates $\left(y_{1}, z_{1}\right)$ and $\left(y_{2}, z_{2}\right)$ as shown in Figure 3.1. Take the branch arc length coordinate $s$ such that $s=0$ at $\left(y_{1}, z_{1}\right)$, and $\mathrm{s}=\mathrm{l}_{\mathrm{s}}$ at $\left(\mathrm{y}_{2}, \mathrm{z}_{2}\right)$.

where

$$
1_{s}=\sqrt{\left(y_{2}-y_{1}\right)^{2}+\left(z_{2}-z_{1}\right)^{2}} \text {. }
$$

From Eqs. (2.2), we have

$$
\frac{d \bar{y}}{d s}=\cos \alpha=\frac{y_{2}-y_{1}}{l_{s}}, \quad \frac{d \bar{z}}{d s}=\sin \alpha=\frac{z_{2}-z_{1}}{l_{s}}
$$

which are integrated to give

$$
\begin{aligned}
& \bar{y}=s \frac{\left(y_{2}-y_{1}\right)}{1_{s}}+y_{1}, \\
& \bar{z}=s \frac{\left(z_{2}-z_{1}\right)}{l_{s}}+z_{1} .
\end{aligned}
$$

Substituting Eqs. (3.2) - (3.4) into Eqs. (2.11), and (2.12) for $\zeta=0$, determines the contour coordinates $r_{R}(s)$, and $q_{R}(s)$ for the straight branch. The results are

$$
\begin{aligned}
& r_{R}=\left(y_{1}-y_{R}\right) \frac{\left(z_{2}-z_{1}\right)}{l_{s}}-\left(z_{1}-z_{R}\right) \frac{\left(y_{2}-y_{1}\right)}{l_{s}} \\
& q_{R}(s)=s+\left(y_{1}-y_{R}\right) \frac{\left(y_{2}-y_{1}\right)}{l_{s}}+\left(z_{1}-z_{R}\right) \frac{\left(z_{2}-z_{1}\right)}{1_{s}}
\end{aligned}
$$

Note that normal contour coordinate $r_{R}$ in Eq. (3.5), is independent of $s$. 
For a straight branch the integral in Eq.(2.44) can be evaluated to give the contour warping function as

$$
\bar{\omega}(s)=s\left(\frac{\left(a-z_{R}\right)}{\left(a-z_{1}\right)} r_{R}-\frac{\left(z_{2}-z_{1}\right)}{l_{s}\left(a-z_{1}\right)} c_{1}\right)+c_{1}
$$

where $c_{1}$ is a constant. The evaluation of the constant $c_{1}$ is discussed in the next section. Substituting for $1 / R_{\theta}$ in Eq.(2.51), and using Eq.(2.48), the thickness warping function is written as,

$$
\tilde{\omega}(s)=\frac{-(a-\bar{z}) q_{R}+\bar{\omega} \cos \alpha}{(a-\bar{z})}
$$

For straight branches having constant material properties in each branch, the modulus weighted section properties in Eqs. (2.103) - (2.117) reduce to summations over all branches of the cross-section. These relations are

$$
\begin{aligned}
& E A=\sum_{i=1}^{n} a_{11} I_{1 i} \\
& E S_{y}=\sum_{i=1}^{n} a_{11} I_{2 i}-b_{11} \cos (\alpha) I_{1 i} \\
& E S_{z}=\sum_{i=1}^{n} a_{11} I_{3 i}+b_{11} \sin (\alpha) I_{1 i} \\
& E S_{\omega}=\sum_{i=1}^{n} a_{11} I_{4 i}+b_{11} I_{5 i} \\
& E H=2 \sum_{i=1}^{n} b_{16} I_{6 i}
\end{aligned}
$$




$$
\begin{aligned}
& E I_{y y}=\sum_{i=1}^{n} a_{11} I_{7 i}-2 b_{11} \cos (\alpha) I_{2 i}+d_{11} \cos ^{2}(\alpha) I_{1 i} \\
& E I_{z y}=\sum_{i=1}^{n} a_{11} I_{8 i}+b_{11} \sin (\alpha) I_{2 i}-b_{11} \cos (\alpha) I_{3 i} \\
& -d_{11} \sin (\alpha) \cos (\alpha) I_{1 \mathrm{i}} \\
& E I_{\omega y}=\sum_{i=1}^{n} a_{11} I_{9 i}+b_{11} I_{10 i}-b_{11} \cos (\alpha) I_{4 i}-d_{11} \cos (\alpha) I_{5 i} \\
& E_{c}=2 \sum_{i=1}^{n} b_{16} I_{11 i}-d_{16} \cos (\alpha) I_{6 i} \\
& E I_{z z}=\sum_{i=1}^{n} a_{11} I_{12 i}+2 b_{11} \sin (\alpha) I_{3 i}+d_{11} \sin ^{2}(\alpha) I_{1 i} \\
& E I_{\omega z}=\sum_{i=1}^{n} a_{11} I_{13 i}+b_{11} I_{14 i}+b_{11} \sin (\alpha) I_{4 i}-d_{11} \sin (\alpha) I_{5 i} \\
& E H_{s}=2 \sum_{i=1}^{n} b_{16} I_{15 i}+d_{16} \sin (\alpha) I_{6 i} \\
& E I_{\omega \omega}=\sum_{i=1}^{n} a_{11} I_{16 i}+2 b_{11} I_{17 i}+d_{11} I_{18 i} \\
& \mathrm{EH}_{\mathrm{q}}=2 \sum_{\mathrm{i}=1}^{\mathrm{n}} \mathrm{b}_{16} \mathrm{I}_{19 \mathrm{i}}+\mathrm{d}_{16} \mathrm{I}_{20 \mathrm{i}} \\
& G J=4 \sum_{i=1}^{n} d_{66} I_{21 i}
\end{aligned}
$$

where $\mathrm{I}_{1 \mathrm{i}}$, through $\mathrm{I}_{21 \mathrm{i}}$, represent twenty one geometric branch integrals which are defined 
by

$$
\begin{aligned}
& I_{1 i}=\int_{0}^{1_{s i}} z b_{t} d s \\
& I_{2 i}=\int_{0}^{1_{s i}}\left(\bar{z}-z_{R}\right) z b_{t} d s \\
& I_{3 i}=\int_{0}^{1_{s i}}\left(\bar{y}-y_{R}\right) z b_{t} d s \\
& I_{4 i}=\int_{0}^{1_{s i}} \bar{\omega} z b_{t} d s \\
& I_{5 i}=\int_{0}^{1_{s i}} \tilde{\omega} z b_{t} d s \\
& I_{6 i}=\int_{0}^{1_{s i}}\left(z b_{t}\right)^{2} d s \\
& I_{7 i}=\int_{0}^{1_{s i}}\left(\bar{z}-z_{R}\right)^{2} z b_{t} d s \\
& I_{8 i}=\int_{0}^{1_{s i}}\left(\bar{z}-z_{R}\right)\left(\bar{y}-y_{R}\right) z b_{t} d s \\
& I_{12 i}=\int_{0}^{s_{s i}}\left(\bar{y}-y_{R}\right)^{2} z b_{t} d s \\
& I_{9 i}=\int_{0}^{1_{s i}}\left(\bar{z}-z_{R}\right) \bar{\omega} z b_{t} d s \\
& I_{10 i}=\int_{0}^{s_{s i}}\left(\bar{y}-y_{R}\right) \bar{\omega} z b_{t} d s \\
& s_{s i} \\
& I_{s i}
\end{aligned}
$$




$$
\begin{aligned}
& I_{14 i}=\int_{0}^{1_{s i}}\left(\bar{y}-y_{R}\right) \tilde{\omega} z_{t} d s \\
& I_{15 i}=\int_{0}^{l_{s i}}\left(\bar{y}-y_{R}\right)\left(z b_{t}\right)^{2} d s \\
& I_{16 i}=\int_{0}^{l_{s i}}(\bar{\omega})^{2} z b_{t} d s \\
& I_{17 i}=\int_{0}^{l_{s i}} \bar{\omega} \tilde{\omega} z_{t} d s \\
& I_{18 i}=\int_{0}^{s_{s i}}(\tilde{\omega})^{2} z b_{t} d s \\
& I_{19 i}=\int_{0}^{s_{s i}} \bar{\omega}\left(z b_{t}\right)^{2} d s \\
& I_{20 i}=\int_{0}^{l_{s i}} \tilde{\omega}\left(z b_{t}\right)^{2} d s \\
& I_{21 i}=\int_{0}^{l_{s i}}\left(z b_{t}\right)^{3} d s
\end{aligned}
$$

The term $z b_{t}$ appearing in the integrands of Eqs.(3.24) - (3.44), is defined by

$$
\mathrm{zb}_{\mathrm{t}}=\frac{\left(\mathrm{a}-\mathrm{z}_{\mathrm{R}}\right)}{(\mathrm{a}-\overline{\mathrm{z}}(\mathrm{s}))}
$$

An approximation is used for computer evaluation of the integral Eqs.(3.24) - (3.44). This approximation involves the term $\mathrm{zb}_{\mathfrak{t}}$ of Eq.(3.45). When Eq.(3.45) is integrated with respect to $s$, with $\bar{z}$ (s) given by Eq.(3.4), the resulting expression is

$$
\int z b_{t} d s= \begin{cases}-\frac{\left(a-z_{R}\right)}{\sin \alpha} \ln \left(a-s \sin \alpha-z_{1}\right) & ,(\alpha \neq 0) \\ \frac{\left(a-z_{R}\right)}{\left(a-z_{1}\right)} s & ,(\alpha=0)\end{cases}
$$


The difficulty is associated with numerical evaluation of Eqn.(3.46). This equation requires switching functions based on the value of the contour angle $\alpha$, to avoid a numerical singularity in the first of Eqs.(3.46), as $\alpha$ approaches zero. The switch is avoided by using a series approximation for Eq.(3.45).The term $z_{t}$ is re-written as $\left(1-z_{R} / a\right)(1-\bar{z} / a)^{-1}$, where $\left(1-z_{R} / a\right)$ is not a function of the shell coordinate $s$. A series approximation for $(1-\bar{z} / a)^{-1}$, is shown in $\mathrm{Eq}(3.47)$.

$$
\left(1-\frac{\bar{z}}{a}\right)^{-1} \cong\left\{1+\frac{\bar{z}}{\mathrm{a}}+\left(\frac{\bar{z}}{\mathrm{a}}\right)^{2}+\left(\frac{\bar{z}}{\mathrm{a}}\right)^{3}+0\left(\bar{z}^{4}\right)\right\}, \quad(|\bar{z}|<1)
$$

\subsection{EVALUATION OF THE CONTOUR WARPING FUNCTION}

Prior to calculation of the torsional section properties, care must be taken to ensure that $\bar{\omega}$ is continuous at the cross-sectional junctions. In general, $\bar{\omega}$ is not a continuous function of arc length coordinate $s$, and its derivative will not exist at the junctions. The constant $c_{1}$ in Eq. (3.7), is unique for each branch. For evaluation purposes, $c_{1}$ can be considered as having two components. That is, $c_{1}=c_{1 a}+c_{b}$. The $c_{1 a}$ term is used to satisfy the continuity requirement of the warping function $\bar{\omega}$ at the junctions, and is in general different for each cross-section branch. The $c_{b}$ term is the same for all section branches, and is used to establish the location of the principal contour origin.

The principal contour origin is defined by the condition

$$
\mathrm{ES}_{\omega}=\int\left(\left(\mathrm{a}_{11} \bar{\omega}+\mathrm{b}_{11} \tilde{\omega}\right)\left(\frac{\mathrm{a}-\mathrm{z}_{\mathrm{R}}}{\mathrm{a}-\overline{\mathrm{z}}}\right)\right)=0
$$

which means that the circumferential axial force is de-coupled from the warping strain in 
the material law, Eq. (2.102). Eq. (3.48) is satisfied by the choice of the contour warping function, since the thickness warping function depends on it via Eq. (2.51). The warping functions for the $i^{\text {th }}$ branch are

$$
\begin{aligned}
& \bar{\omega}\left(s_{i}\right)=\bar{\omega}^{*}\left(s_{i}\right)+c_{b}\left(\frac{a-\bar{z}}{a-z_{1}}\right)_{i} \\
& \tilde{\omega}\left(s_{i}\right)=\tilde{\omega}^{*}\left(s_{i}\right)+c_{b}\left(\frac{\cos \alpha}{a-z_{1}}\right)_{i}
\end{aligned}
$$

where,

$$
\begin{aligned}
& \bar{\omega}^{*}\left(s_{i}\right)=s_{i}\left(\frac{\left(a-z_{R}\right)}{\left(a-z_{1}\right)} r_{R}-\frac{\left(z_{2}-z_{1}\right)}{1_{s}\left(a-z_{1}\right)} c_{1 a}\right)_{i}+c_{1 a} \\
& \tilde{\omega}^{*}\left(s_{i}\right)=\left(\frac{-(a-\bar{z}) q_{R}+\bar{\omega}^{*} \cos \alpha}{(a-\bar{z})}\right)_{i}
\end{aligned}
$$

and $0 \leq s_{i} \leq 1_{s i}$. Substituting Eqs.(3.49), and (3.50), into Eqs.(3.27), and (3.28), and in turn substituting these results into Eq. (3.12) we get

$$
\begin{aligned}
E S_{\omega}= & \sum_{i=1}^{n} a_{11} I_{4 i}^{*}+b_{11} I_{5 i}^{*} \\
& +c_{b} \sum_{i=1}^{n}\left\{a_{11}\left(\frac{a-\bar{z}}{a-z_{1}}\right)_{i}+b_{11}\left(\frac{\cos \alpha}{a-z_{1}}\right)\right\}
\end{aligned}
$$

where

$$
\begin{aligned}
& I_{4 i}^{*}=\int_{0}^{1_{s i}} \omega^{*} z b_{t} d s \\
& I_{5 i}^{*}=\int_{0}^{1_{s i}} \tilde{\omega}^{*} z b_{t} d s
\end{aligned}
$$


The integrals in Eqs. (3.54) and (3.55) are evaluated after constants $c_{1 \mathrm{a}}$ are determined from continuity of the contour warping at the junctions. Setting $\mathrm{ES}_{\omega}=0$ in Eq.(3.53), gives the equation from which the constant $c_{b}$ can be found. This procedure establishes the principal contour origin, and is illustrated for a section composed of two branches in Figure 3.2 .

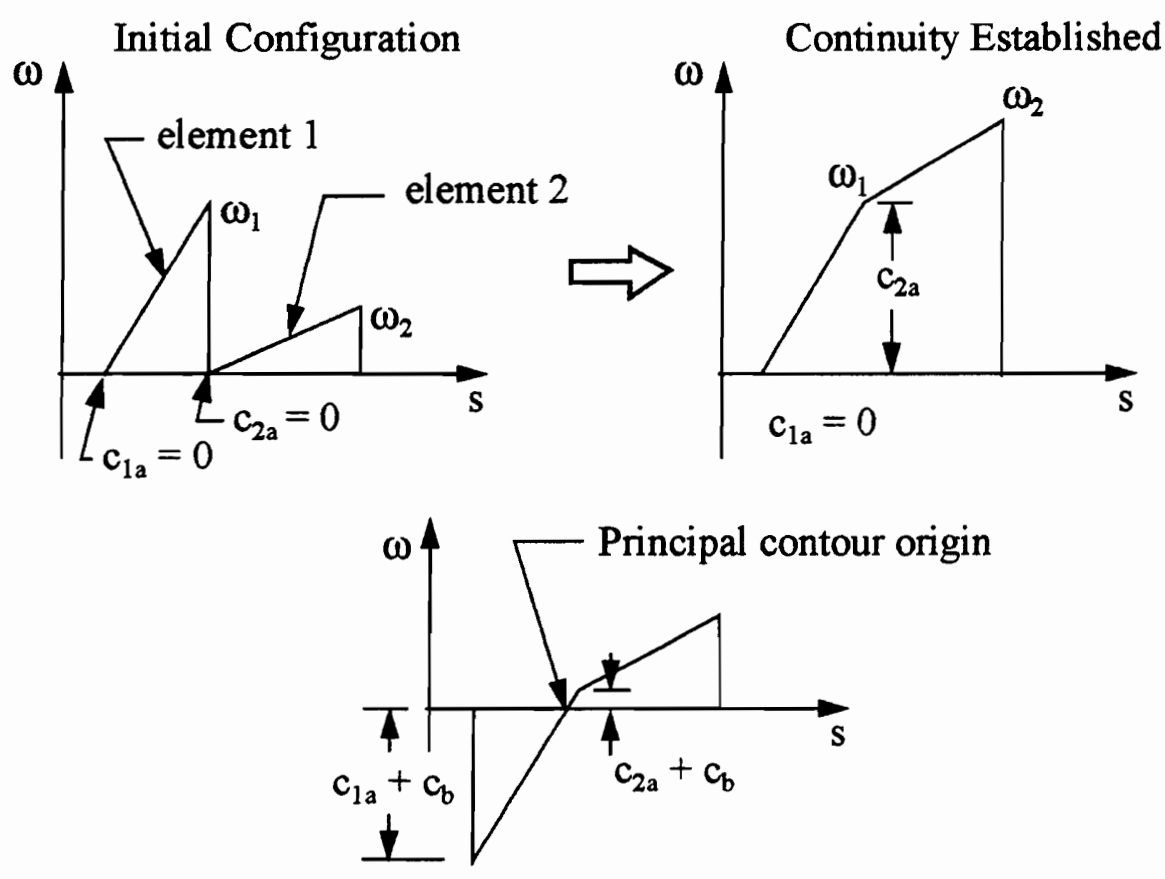

Figure 3.2 Location of principal contour origin.

\section{4 PROGRAM VERIFICATION}

A computer program was written to calculate the section properties using the methodology described in this chapter. Section properties calculated by the program were verified by comparing them to properties obtained, or calculated independently. The bending properties for isotropic beams are easily verified since many formulas and programs for calculating these properties are available. For curved beam torsional properties, and the effects of composite materials few examples are available for comparison. The following is a brief description of the effort to verify results obtained from the program. 
Formulas for torsional properties of several cross-section geometries are given by Roark ${ }^{l}$ ${ }^{54}$. These were used as a basic check of torsional properties for isotropic sections. An "I" section with unequal legs, a "Z" section, a "channel" section, and an "angle" section were used for this check. The properties calculated by formulas were compared to the program output and found to be in close agreement. Section properties were also calculated and compared to the results published by Collins and Johnson [ ${ }^{49}$ ], for a composite " $\mathrm{T}$ " section, and a composite " $\mathrm{J}$ " section. These were also found to be in close agreement.

To ensure that nothing unusual was occurring, the material properties for a composite "I" section were varied such that no change in section properties would be expected. The section was first calculated using a single layer of isotropic material for each branch. The single layer in each branch, was then divided into two layers of isotropic material for each cross section branch, and the properties were calculated again yielding identical results. Next, the section was checked by switching the $0^{\circ}$ and $90^{\circ}$ plies, and the $E_{1}$, and $E_{2} \bmod -$ uli. As expected this had no effect on the properties. Finally, $+45^{\circ}$, and $-45^{\circ}$ plies were switched, and this also had no effect on the properties.

From these calculations it was initially verified that the section properties program performs as expected for a variety of different types of cross sections. Properties calculated for both symmetric and unsymmetric cross sections were verified. Changes made to material lay up and ply orientation had no unexpected results. The lack of available data for comparison purposes makes $100 \%$ verification of the programs behavior difficult. 


\section{CHAPTER 4}

\section{FINITE ELEMENT DEVELOPMENT}

\subsection{INTRODUCTION}

In this chapter, the thin walled laminated composite curved beam theory developed in chapter two is implemented by the displacement based finite element method. The principle of virtual work is used to develop the element stiffness matrix and force vector in a local curvilinear coordinate system. A transformation matrix is provided so that analysis can be conducted in a global cartesian coordinate system.

\subsection{ELEMENT FORMULATION}

The displacement based finite element for thin-walled curved beam theory is developed beginning with the expression for internal virtual work given by Eq.(2.63).

$$
\delta U=\int_{\theta_{1}}^{\theta_{2}}\{\delta \varepsilon\}^{\mathrm{T}}\{\mathrm{N}\}\left(\mathrm{a}-\mathrm{z}_{\mathrm{R}}\right) \mathrm{d} \theta
$$


where the 5 by 1 vector of bar resultants is

$$
\{N\}=\left\{\begin{array}{c}
N_{R} \\
M_{y R} \\
M_{z R} \\
M_{\omega R} \\
T_{s R}
\end{array}\right\}
$$

and the 5 by 1 vector of bar strains is

$$
\{\varepsilon\}=\left\{\begin{array}{c}
\varepsilon_{\mathrm{R}} \\
\kappa_{\mathrm{yR}} \\
\kappa_{\mathrm{zR}} \\
\tau_{\mathrm{R}}^{\prime} /\left(\mathrm{a}-\mathrm{z}_{\mathrm{R}}\right) \\
\tau_{\mathrm{R}}
\end{array}\right\} \text {, }
$$

The element bar resultants, and the element bar strains are defined by Eqs.(2.66)-(2.70), (2.57)-(2.59), and Eq.(2.42), respectively. The constitutive relation, Eq. (2.102) is reintroduced as

$$
\{N\}=[C]\{\varepsilon\},
$$

where the 5 by 5 stiffness matrix is

$$
[C]=\left[\begin{array}{ccccc}
E A & E_{y} & -E_{z} & -E_{\omega} & E H \\
E_{y} & E_{y y} & -E_{z y} & -E_{\omega y} & E_{c} \\
-E_{z} & -E I_{z y} & E_{z z} & E_{\omega z} & -E_{s} \\
-E_{\omega} & -E I_{\omega y} & E_{\omega z} & E_{\omega \omega} & -E_{q} \\
E H & E_{c} & -E_{s} & -E H_{q} & G J
\end{array}\right]
$$


Substituting Eq.(4.4) into Eq.(4.1), the expression for internal virtual work given by Eq.(4.1) is written as

$$
\delta U=\int_{\theta_{1}}^{\theta_{2}}\{\delta \varepsilon\}^{T}[C]\{\varepsilon\}\left(a-z_{R}\right) d \theta,
$$

To continue the finite element development, element bar strains $\{\varepsilon\}$ must be expressed in terms of nodal degrees of freedom. This is accomplished by approximating the element displacements and rotations as functions of the nodal degrees of freedom. This approximate relationship is expressed as

$$
\{\mathrm{u}(\theta)\}=[\mathrm{H}(\boldsymbol{\theta})]\{\hat{\mathrm{u}}\},
$$

where $[\mathrm{H}]$ is a matrix of interpolation functions relating the displacement vector $\{\mathrm{u}\}$, to the nodal degrees of freedom in $\{\hat{\mathbf{u}}\}$. Recall from chapter 2 , that the displacements and rotations for the thin-walled curved bar are $\mathrm{U}_{\mathrm{R}}, \mathrm{V}_{\mathrm{R}}, \mathrm{W}_{\mathrm{R}}, \Phi_{\mathrm{XR}}, \Phi_{\mathrm{yR}}, \Phi_{\mathrm{ZR}}$, and $\tau_{\mathrm{R}}$ (see Figure 2.7), where the subscript $R$ indicates that these are calculated at the cross section reference point R. Using Eqs.(2.15), (2.16), and (2.42), $\Phi_{y R}, \Phi_{2 R}$, and $\tau_{R}$, can be expressed in terms of the remaining displacement components and their derivatives. Therefore, the independent displacements and rotations for the thin walled curved beam are given by the 4-by-1 vector

$$
\{u(\theta)\}=\left\{\begin{array}{c}
\mathrm{U}_{\mathrm{R}}(\theta) \\
\mathrm{V}_{\mathrm{R}}(\theta) \\
\mathrm{W}_{\mathrm{R}}(\theta) \\
\Phi_{\mathrm{XR}}(\theta)
\end{array}\right\},
$$

The curved beam strains are obtained by differentiating and combining the components of $\{u\}$ given by Eq.(4.8). Recall that the radius of curvature is held constant within an ele- 
ment. The strain-displacement equations developed in Chapter 2 are collected here for convenience

$$
\begin{array}{ll}
\varepsilon_{\mathrm{R}}=\frac{\mathrm{U}_{\mathrm{R}}^{\prime}-\mathrm{W}_{\mathrm{R}}}{\left(\mathrm{a}-\mathrm{z}_{\mathrm{R}}\right)} & =\frac{\mathrm{U}_{\mathrm{R}}^{\prime}}{\left(\mathrm{a}-\mathrm{z}_{\mathrm{R}}\right)}-\frac{\mathrm{W}_{\mathrm{R}}}{\left(\mathrm{a}-\mathrm{z}_{\mathrm{R}}\right)} \\
\mathrm{K}_{\mathrm{yR}}=\frac{\Phi_{\mathrm{yR}}^{\prime}}{\left(\mathrm{a}-\mathrm{z}_{\mathrm{R}}\right)} & =-\frac{\mathrm{W}_{\mathrm{R}}^{\prime \prime}}{\left(\mathrm{a}-\mathrm{z}_{\mathrm{R}}\right)^{2}}-\frac{\mathrm{U}_{\mathrm{R}}^{\prime}}{\left(\mathrm{a}-\mathrm{z}_{\mathrm{R}}\right)^{2}} \\
\kappa_{\mathrm{z} \mathrm{R}}=\frac{\Phi_{\mathrm{zR}}^{\prime}+\Phi_{\mathrm{xR}}}{\left(\mathrm{a}-\mathrm{z}_{\mathrm{R}}\right)} & =\frac{\mathrm{V}_{\mathrm{R}}^{\prime \prime}}{\left(\mathrm{a}-\mathrm{z}_{\mathrm{R}}\right)^{2}}+\frac{\Phi_{\mathrm{xR}}}{\left(\mathrm{a}-\mathrm{z}_{\mathrm{R}}\right)} \\
\frac{\tau_{\mathrm{R}}^{\prime}}{\left(\mathrm{a}-\mathrm{z}_{\mathrm{R}}\right)}=\frac{\Phi_{\mathrm{xR}}^{\prime \prime}-\Phi_{\mathrm{zR}}^{\prime}}{\left(\mathrm{a}-\mathrm{z}_{\mathrm{R}}\right)^{2}} & =\frac{\Phi_{\mathrm{xR}}^{\prime \prime}}{\left(\mathrm{a}-\mathrm{z}_{\mathrm{R}}\right)^{2}}-\frac{\mathrm{V}_{\mathrm{R}}^{\prime \prime}}{\left(\mathrm{a}-\mathrm{z}_{\mathrm{R}}\right)^{3}} \\
\tau_{\mathrm{R}}=\frac{\Phi_{\mathrm{xR}}^{\prime}-\Phi_{\mathrm{z} R}}{\left(\mathrm{a}-\mathrm{z}_{\mathrm{R}}\right)} & =\frac{\Phi_{\mathrm{xR}}^{\prime}}{\left(\mathrm{a}-\mathrm{z}_{\mathrm{R}}\right)}-\frac{\mathrm{V}_{\mathrm{R}}^{\prime}}{\left(\mathrm{a}-\mathrm{z}_{\mathrm{R}}\right)^{2}}
\end{array}
$$

The relationship between strains and nodal degrees of freedom is commonly written as

$$
\{\varepsilon\}=[B]\{\hat{u}\},
$$

in which $[B]$ is the 5-by-4 strain-displacement matrix related to the interpolation matrix by

$$
[B]=[L][H(\theta)],
$$

In Eq.(4.11), [L] is a matrix of differential operators determined from Eqs.(4.9), such that $\{\varepsilon\}=[L]\{u\}$, with $\{\varepsilon\}$ given by Eq.(4.3) and $\{u\}$ given by Eq.(4.8). For the cylindrical coordinate system, the 5 -by- 4 operator matrix $[\mathrm{L}]$ is 


$$
[L]=\left[\begin{array}{cccc}
\frac{1}{\left(a-z_{R}\right)} \frac{d}{d \theta} & 0 & \frac{-1}{\left(a-z_{R}\right)} & 0 \\
\frac{-1}{\left(a-z_{R}\right)^{2}} \frac{d}{d \theta} & 0 & \frac{-1}{\left(a-z_{R}\right)^{2}} \frac{d^{2}}{d \theta^{2}} & 0 \\
0 & \frac{1}{\left(a-z_{R}\right)^{2}} \frac{d^{2}}{d \theta^{2}} & 0 & \frac{1}{\left(a-z_{R}\right)} \\
0 & \frac{-1}{\left(a-z_{R}\right)^{3}} \frac{d^{2}}{d \theta^{2}} & 0 & \frac{1}{\left(a-z_{R}\right)^{2}} \frac{d^{2}}{d \theta^{2}} \\
0 & \frac{-1}{\left(a-z_{R}\right)^{2}} \frac{d}{d \theta} & 0 & \frac{1}{\left(a-z_{R}\right)} \frac{d}{d \theta}
\end{array}\right]
$$

To accommodate the situation for which adjacent beam elements do not have a common center of curvature, the independent variable $\theta$, is changed to changed to the arc length coordinate $x$. Substituting $d x=\left(a-z_{R}\right) d \theta$, the following relations are obtained.

$$
\begin{aligned}
& \frac{d}{d \theta}=\frac{d}{d x}\left(\frac{d x}{d \theta}\right)=\left(a-z_{R}\right) \frac{d}{d x} \\
& \frac{d^{2}}{d \theta^{2}}=\frac{d}{d x}\left(\left(a-z_{R}\right) \frac{d}{d x}\right) \frac{d x}{d \theta}=\left(a-z_{R}\right)^{2} \frac{d^{2}}{d x^{2}}
\end{aligned}
$$

Substituting Eqs.(4.13) and (4.14) into Eq.(4.12), the operator matrix [L], in terms of arc length coordinate $\mathrm{x}$ is 


$$
[L]=\left[\begin{array}{cccc}
\frac{d}{d x} & 0 & \frac{-1}{\left(a-z_{R}\right)} & 0 \\
\frac{-1}{\left(a-z_{R}\right)} \frac{d}{d x} & 0 & \frac{-d^{2}}{d x^{2}} & 0 \\
0 & \frac{d^{2}}{d x^{2}} & 0 & \frac{1}{\left(a-z_{R}\right)} \\
0 & \frac{-1}{\left(a-z_{R}\right)} \frac{d^{2}}{d x^{2}} & 0 & \frac{d^{2}}{d x^{2}} \\
0 & \frac{-1}{\left(a-z_{R}\right)} \frac{d}{d x} & 0 & \frac{d}{d x}
\end{array}\right]
$$

With [L] defined by Eq.(4.15), and [B] defined by Eq.(4.11), Eq.(4.10) is substituted into Eq.(4.6), to obtain the expression for internal virtual work as

$$
\delta U=\int_{x_{1}}^{x_{2}}\{\delta \hat{u}\}^{T}[B]^{T}[C][B]\{\hat{u}\} d x
$$

Since the nodal displacements $\{\hat{\mathrm{u}}\}$, are independent of " $\mathrm{x}$ ", these terms can be brought outside the integral of Eq.(4.16). Including the contribution from external virtual work, the expression for element equilibrium can now be written as

$$
\begin{aligned}
& \{\delta \hat{u}\}^{T} \int_{x_{1}}^{x_{2}}[B]^{T}[C][B] d x\{\hat{u}\} \\
& =\{\delta \hat{u}\}^{T}\left[\int_{x_{1}}^{x_{2}}[H]^{T}\left\{f_{d}\right\} d x+\{F\}\right]
\end{aligned}
$$

where $\left\{f_{d}\right\}$ represents the element distributed load intensities at each node, and $\{F\}$ represents the concentrated nodal forces. 
The curved beam element stiffness matrix can now be written as

$$
\left[K^{e}\right]=\int_{x_{1}}^{x_{2}}[B]^{T}[C][B] d x
$$

and the element force vector can be written as

$$
\left\{f^{e}\right\}=\int_{x_{1}}^{x_{2}}[H]^{T}\left\{f_{d}\right\} d x+\{F\}
$$

\subsection{DISPLACEMENT APPROXIMATION}

The interpolation matrix [H] was introduced in Eq.(4.7), and remains to be defined. This matrix of interpolation functions is used to approximate each of the displacements $\{u\}$, of Eq.(4.8) as $\mathrm{u}_{\mathrm{i}}(\mathrm{x}) \cong \sum \hat{\mathrm{u}}_{\mathrm{j}} \mathrm{H}_{\mathrm{ij}}(\mathrm{x}), \mathrm{j}=1, \mathrm{n}$. Element $\mathrm{H}_{\mathrm{ij}}(\mathrm{x})$ represents the $\mathrm{j}^{\text {th }}$ interpolation function for the displacement component $u_{i}$, and $\hat{u}_{j}$ represents the $j^{\text {th }}$ nodal displacement component. The definition of $[\mathrm{H}]$ requires that nodal displacement components for the curved beam finite element be identified. The nodal displacement components required at the element boundaries are determined from an examination of Eqs.(4.9). In the internal virtual work functional the highest order derivative of $U_{R}$ is first, and the highest order derivatives of $V_{R}, W_{R}$, and $\Phi_{X R}$ are second. For kinematic admissibility of the displacement field components, $\mathrm{U}_{\mathrm{R}}$ must have $\mathrm{C}^{0}$ continuity, and $\mathrm{V}_{\mathrm{R}}, \mathrm{W}_{\mathrm{R}}$, and $\Phi_{\mathrm{XR}}$ must have $C^{1}$ continuity between elements, and within elements. Hence, $U_{R}, V_{R}, V_{R}^{\prime}, W_{R}$, $W_{R}^{\prime} \Phi_{x R}$, and $\Phi_{x R}^{\prime}$ are the degrees of freedom at the end nodes of the element, and $\{\hat{u}\}$ must be a least 14-by-1.

For the polynomial displacement interpolation of the curved beam element to be capable 
of representing rigid body motion, higher degree polynomials are recommended (see Dawe ${ }^{[55][56]}$ ) The work by Dawe suggests that high order interpolation functions insure a higher degree of accuracy, and rapid convergence. A three node curved beam element using $5^{\text {th }}$ order interpolation polynomials for $U_{R}, V_{R}, W_{R}$, and $\Phi_{X R}$ was selected for evaluation. A diagram of this element is shown in Figure 4.1.

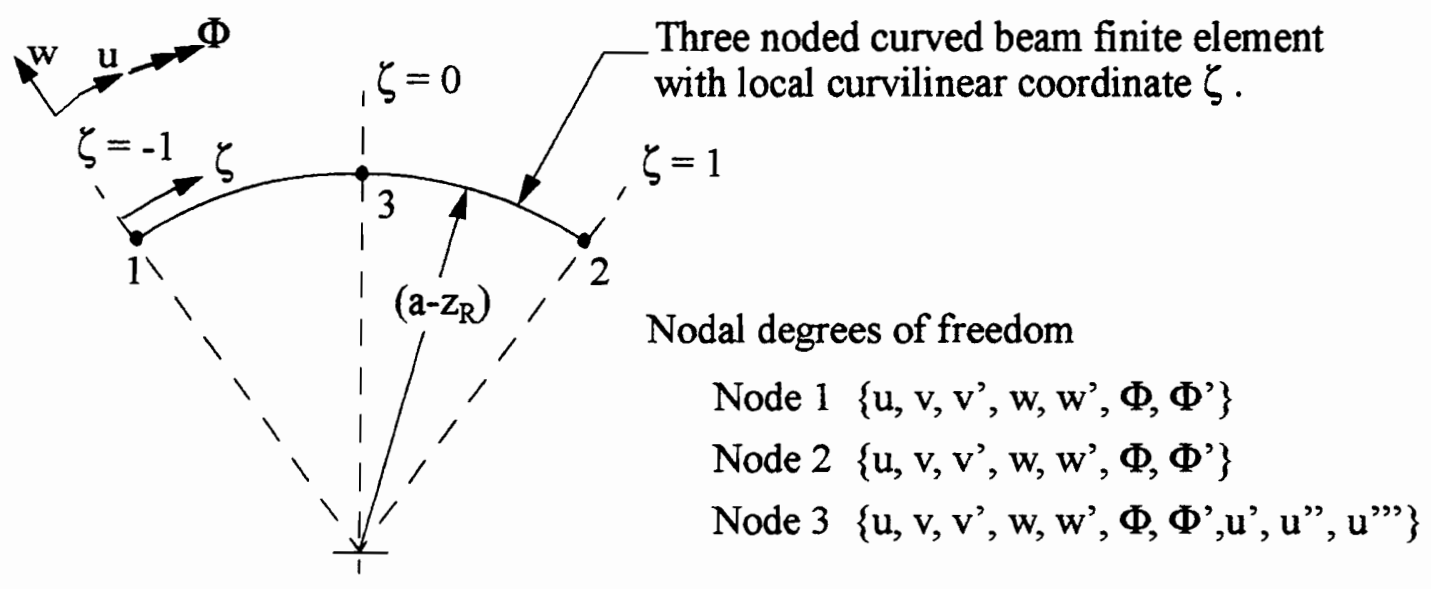

Center of curvature

Figure 4.1 Three node curved beam element

For the axial degree of freedom $U_{R}$, derivatives need not be continuous between elements. This means that the remaining four degrees of freedom required for quintic polynomial interpolation must be placed at the center node. For $V_{R}, W_{R}$, and $\Phi_{x R}$, the displacement and its first derivative will be represented at each node. Two sets of Hermite polynomial interpolation functions are required. One set for $U_{R}$, and another set for $V_{R}, W_{R}$, and $\Phi_{x R}$. The element is developed in local coordinates $\zeta=-1$ to 1 , to facilitate numerical integration using Gauss quadrature. This choice of coordinates also simplifies the form of the interpolation polynomials. For $U_{R}(\zeta)$, the interpolation takes the following form.

$$
\begin{aligned}
\mathrm{u}(\zeta) & =\hat{\mathrm{u}}_{1} \Psi_{\mathrm{u} 1}(\zeta)+\hat{\mathrm{u}}_{2} \Psi_{\mathrm{u} 2}(\zeta)+\hat{\mathrm{u}}_{3} \Psi_{\mathrm{u} 3}(\zeta)+\hat{\mathrm{u}}_{3}^{\prime} \Psi_{\mathrm{u} 4}(\zeta) \\
& +\hat{\mathrm{u}}_{3}{ }_{3} \Psi_{\mathrm{u} 5}(\zeta)+\hat{\mathrm{u}}^{\prime{ }^{\prime}}{ }_{3} \Psi_{\mathrm{u} 6}(\zeta)
\end{aligned}
$$


where

$$
\begin{aligned}
& \Psi_{\mathrm{u} 1}(\zeta)=\frac{\zeta^{4}}{2}-\frac{\zeta^{5}}{2}, \\
& \Psi_{\mathrm{u} 2}(\zeta)=\frac{\zeta^{4}}{2}+\frac{\zeta^{5}}{2}, \\
& \Psi_{\mathrm{u} 3}(\zeta)=1-\zeta^{4}, \\
& \Psi_{\mathrm{u} 4}(\zeta)=\zeta-\zeta^{5}, \\
& \Psi_{\mathrm{u} 5}(\zeta)=\frac{\zeta^{2}}{2}-\frac{\zeta^{4}}{2}, \\
& \Psi_{\mathrm{u} 6}(\zeta)=\frac{\zeta^{3}}{6}-\frac{\zeta^{5}}{6},
\end{aligned}
$$

and for $V_{R}, W_{R}$, and $\Phi_{x R}$, the interpolation takes the form

$$
\begin{aligned}
\mathrm{v}(\zeta) & =\hat{\mathrm{v}}_{1} \Psi_{\mathrm{v} 1}(\zeta)+\hat{\mathrm{v}}_{1}^{\prime} \Psi_{\mathrm{v} 2}(\zeta)+\hat{\mathrm{v}}_{2} \Psi_{\mathrm{v} 3}(\zeta)+\hat{\mathrm{v}}_{2}^{\prime} \Psi_{\mathrm{v} 4}(\zeta) \\
& +\hat{\mathrm{v}}_{3} \Psi_{\mathrm{v} 5}(\zeta)+\hat{\mathrm{v}}_{3}^{\prime} \Psi_{\mathrm{v} 6}(\zeta)
\end{aligned}
$$

where

$$
\begin{aligned}
& \Psi_{\mathrm{v} 1}(\zeta)=\zeta^{2}-\frac{5 \zeta^{3}}{4}-\frac{\zeta^{4}}{2}+\frac{3 \zeta^{5}}{4}, \\
& \Psi_{\mathrm{v} 2}(\zeta)=\frac{\zeta^{2}}{4}-\frac{\zeta^{3}}{4}-\frac{\zeta^{4}}{4}+\frac{\zeta^{5}}{4}, \\
& \Psi_{\mathrm{v} 3}(\zeta)=\zeta^{2}+\frac{5 \zeta^{3}}{4}-\frac{\zeta^{4}}{2}-\frac{3 \zeta^{5}}{4}, \\
& \Psi_{\mathrm{v} 4}(\zeta)=-\frac{\zeta^{2}}{4}-\frac{\zeta^{3}}{4}+\frac{\zeta^{4}}{4}+\frac{\zeta^{5}}{4}, \\
& \Psi_{\mathrm{v} 5}(\zeta)=1-2 \zeta^{2}+\zeta^{4},
\end{aligned}
$$




$$
\Psi_{\mathrm{v} 6}(\zeta)=\zeta-2 \zeta^{3}+\zeta^{5}
$$

The 24-degree-of-freedom nodal displacement vector for the element is

$$
\begin{aligned}
\{\hat{\mathrm{u}}\}^{\mathrm{T}}= & \left\{\hat{\mathrm{u}}_{1} \hat{\mathrm{v}}_{1} \hat{\mathrm{v}}_{1}^{\prime} \hat{\mathrm{w}}_{1} \hat{\mathrm{w}}_{1}^{\prime} \hat{\Phi}_{\mathrm{x} 1} \hat{\Phi}_{\mathrm{x} 1}^{\prime} \hat{\mathrm{u}}_{2} \hat{\mathrm{v}}_{2} \hat{\mathrm{v}}_{2}^{\prime} \hat{\mathrm{w}}_{2} \hat{\mathrm{w}}_{2}^{\prime} \hat{\Phi}_{\mathrm{x} 2} \hat{\Phi}_{\mathrm{x} 2}^{\prime}\right. \\
& \left.\hat{\mathrm{u}}_{3} \hat{\mathrm{v}}_{3} \hat{\mathrm{v}}_{3}^{\prime} \hat{\mathrm{w}}_{3} \hat{\mathrm{w}}_{3}^{\prime} \hat{\Phi}_{\mathrm{x} 3} \hat{\Phi}_{\mathrm{x} 3}^{\prime} \hat{\mathrm{u}}_{3}^{\prime} \hat{\mathrm{u}}_{3}^{\prime \prime} \hat{\mathrm{u}}_{3}^{\prime \prime \prime}\right\}
\end{aligned}
$$

The 4-by-24 interpolation matrix for the three node element described above can now be written in partitioned form as

$$
[\mathrm{H}]=\left[\mathrm{H}_{1} i^{\prime} \mathrm{H}_{2}: \mathrm{H}_{3} i_{4} \mathrm{H}_{4}\right]
$$

where

$$
\begin{aligned}
{\left[\mathrm{H}_{1}\right]=} & {\left[\begin{array}{ccccccc}
\Psi_{\mathrm{u} 1} & 0 & 0 & 0 & 0 & 0 & 0 \\
0 & \Psi_{\mathrm{v} 1} & \Psi_{\mathrm{v} 2} & 0 & 0 & 0 & 0 \\
0 & 0 & 0 & \Psi_{\mathrm{w} 1} & \Psi_{\mathrm{w} 2} & 0 & 0 \\
0 & 0 & 0 & 0 & 0 & \Psi_{\Phi 1} & \Psi_{\Phi 2}
\end{array}\right], } \\
{\left[\mathrm{H}_{2}\right]=} & {\left[\begin{array}{ccccccc}
\Psi_{\mathrm{u} 2} & 0 & 0 & 0 & 0 & 0 & 0 \\
0 & \Psi_{\mathrm{v} 3} & \Psi_{\mathrm{v} 4} & 0 & 0 & 0 & 0 \\
0 & 0 & 0 & \Psi_{\mathrm{w} 3} & \Psi_{\mathrm{w} 4} & 0 & 0 \\
0 & 0 & 0 & 0 & 0 & \Psi_{\Phi 3} & \Psi_{\Phi 4}
\end{array}\right], } \\
{\left[\mathrm{H}_{3}\right]=} & {\left[\begin{array}{ccccccc}
\Psi_{\mathrm{u} 3} & 0 & 0 & 0 & 0 & 0 & 0 \\
0 & \Psi_{\mathrm{v} 5} & \Psi_{\mathrm{v} 6} & 0 & 0 & 0 & 0 \\
0 & 0 & 0 & \Psi_{\mathrm{w} 5} \Psi_{\mathrm{w} 6} & 0 & 0 \\
0 & 0 & 0 & 0 & 0 & \Psi_{\Phi 5} & \Psi_{\Phi 6}
\end{array}\right], }
\end{aligned}
$$




$$
\left[\mathrm{H}_{4}\right]=\left[\begin{array}{ccc}
\Psi_{\mathrm{u} 4} & \Psi_{\mathrm{u} 5} & \Psi_{\mathrm{u} 6} \\
0 & 0 & 0 \\
0 & 0 & 0 \\
0 & 0 & 0
\end{array}\right]
$$

\section{4 STATIC CONDENSATION}

The internal degrees of freedom associated with node three can be eliminated using static condensation. This is done after the element stiffness matrix has been calculated, and removes the middle node and it's associated ten degrees of freedom in the formulation. The 24 equilibrium equations for the element are written in matrix form as follows.

$$
\left[\mathrm{K}^{\mathrm{e}}\right]\{\hat{\mathrm{u}}\}=\left\{\mathrm{f}^{\mathrm{e}}\right\},
$$

For static condensation, the element stiffness matrix $\left[\mathrm{K}^{\mathrm{e}}\right]$, the nodal displacement vector $\{\hat{u}\}$, and the nodal force vector $\left\{f^{\mathfrak{e}}\right\}$, are partitioned for admitted and condensed degrees of freedom. That is,

$$
\begin{aligned}
& {\left[\mathrm{K}^{\mathrm{e}}\right]=\left[\begin{array}{ll}
{\left[\mathrm{K}_{\mathrm{aa}}\right]} & {\left[\mathrm{K}_{\mathrm{ac}}\right]} \\
{\left[\mathrm{K}_{\mathrm{ca}}\right]} & {\left[\mathrm{K}_{\mathrm{cc}}\right]}
\end{array}\right] \text {, }} \\
& \{\hat{u}\}=\left[\begin{array}{c}
\left\{\hat{u}_{a}\right\} \\
\left\{\hat{u}_{c}\right\}
\end{array}\right], \quad\left\{f^{e^{e}}\right\}=\left[\begin{array}{c}
\left\{R_{a}\right\} \\
\left\{R_{c}\right\}
\end{array}\right] \text {, }
\end{aligned}
$$

where

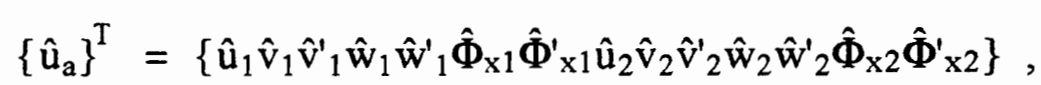




$$
\left\{\hat{u}_{c}\right\}^{T}=\left\{\hat{u}_{3} \hat{v}_{3} \hat{v}_{3}^{\prime} \hat{w}_{3} \hat{w}^{\prime}{ }_{3} \hat{\Phi}_{x 3} \hat{\Phi}_{\times 3}^{\prime} \hat{u}_{3}{ }_{3} \hat{u}^{\prime \prime}{ }_{3} \hat{u}^{\prime \prime \prime}{ }_{3}\right\}
$$

The condensed degrees of freedom $\left\{\hat{\mathrm{u}}_{\mathrm{c}}\right\}$ are obtained in terms of the admitted degrees of freedom $\left\{\hat{\mathrm{u}}_{\mathrm{a}}\right\}$, and the condensed forces $\left\{\mathbf{R}_{\mathrm{c}}\right\}$, by solving the last ten equations in matrix Eq.(4.39) to obtain the following.

$$
\left\{\hat{\mathrm{u}}_{\mathrm{c}}\right\}=\left[\mathrm{K}_{\mathrm{cc}}\right]^{-1}\left[\left\{\mathrm{R}_{\mathrm{c}}\right\}-\left[\mathrm{K}_{\mathrm{ca}}\right]\left\{\hat{\mathrm{u}}_{\mathrm{a}}\right\}\right]
$$

Substituting Eqs.(4.44) into the first fourteen equations of matrix Eq.(4.39), the reduced element stiffness matrix is obtained as

$$
\left[\mathrm{K}_{\mathrm{r}}^{\mathrm{e}}\right]=\left[\mathrm{K}_{\mathrm{aa}}\right]-\left[\mathrm{K}_{\mathrm{ac}}\right]\left[\mathrm{K}_{\mathrm{cc}}\right]^{-1}\left[\mathrm{~K}_{\mathrm{ca}}\right]
$$

and the reduced element force vector is

$$
\left\{\mathrm{f}_{\mathrm{r}}^{\mathrm{e}}\right\}=\left\{\mathrm{R}_{\mathrm{a}}\right\}-\left[\mathrm{K}_{\mathrm{ac}}\right]\left[\mathrm{K}_{\mathrm{cc}}\right]^{-1}\left\{\mathrm{R}_{\mathrm{c}}\right\}
$$

The reduced element stiffness matrix $\left[K_{\mathrm{r}}^{\mathrm{e}}\right]$, is 14 by 14 , and the 14 by 1 reduced nodal displacement vector is $\left\{\hat{\mathrm{u}}_{\mathrm{a}}\right\}^{\mathrm{T}}$.

\subsection{ELEMENT TRANSFORMATION}

The reduced element stiffness matrix given by Eq (4.45) is expressed in the local curvilinear coordinate system. Although many practical curved beam problems can be analyzed, the curvilinear system imposes some limitations on the type of analysis which can be performed. Specifically, the curvilinear system only supports a planar assembly of curved 
beam elements joined tangentially at the ends. To provide for more generality, a global cartesian system is used for analysis. This requires that the element stiffness matrix be transformed to global cartesian coordinates prior to assembly. The global cartesian degrees of freedom are identified in Figure 4.2.

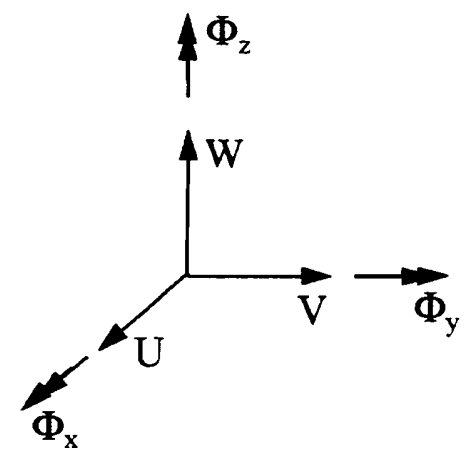

Figure 4.2 Cartesian degrees of freedom

First the fourteen degrees of freedom listed in Eq.(4.42) are related to the generalized beam displacement vector $\left\{\hat{u}_{\mathrm{g}}\right\}$ evaluated at node 1 and at node 2 . For example, at node 1

$$
\left\{\begin{array}{c}
\hat{\mathbf{u}}_{1} \\
\hat{\mathbf{v}}_{1} \\
\hat{\mathbf{v}}_{1}^{\prime} \\
\hat{\mathbf{w}}_{1} \\
\hat{\mathbf{w}}_{1}^{\prime} \\
\hat{\boldsymbol{\Phi}}_{\mathrm{x} 1} \\
\hat{\boldsymbol{\Phi}}_{\mathrm{x} 1}^{\prime}
\end{array}\right\}=\left[\begin{array}{ccccccc}
1 & 0 & 0 & 0 & 0 & 0 & 0 \\
0 & 1 & 0 & 0 & 0 & 0 & 0 \\
0 & 0 & 0 & 0 & 0 & 1 & 0 \\
0 & 0 & 1 & 0 & 0 & 0 & 0 \\
-1 & 0 & 0 & 0 & -1 & 0 & 0 \\
0 & 0 & 0 & 1 & 0 & 0 & 0 \\
0 & 0 & 0 & 0 & 0 & \frac{1}{\left(\mathrm{a}-\mathrm{z}_{\mathrm{R}}\right)} \\
0 & 1
\end{array}\right]\left\{\begin{array}{c}
\hat{U}_{1} \\
\hat{\mathrm{V}}_{1} \\
\hat{\mathrm{W}}_{1} \\
\hat{\boldsymbol{\Phi}}_{\mathrm{x} 1} \\
\hat{\boldsymbol{\Phi}}_{\mathrm{y} 1} \\
\hat{\boldsymbol{\Phi}}_{\mathrm{z} 1} \\
\hat{\tau}_{1}
\end{array}\right\}
$$

or in matrix form 


$$
\left\{\hat{\mathrm{u}}_{\mathrm{a}}^{1}\right\}=\left[\mathrm{T}_{1 \mathrm{e}}\right]\left\{\hat{\mathrm{u}}_{\mathrm{g}}^{1}\right\}
$$

Note that the subscripts "R" on the elements of $\left\{\hat{u}_{\mathrm{g}}\right\}$ in Eq.(4.47) has been dropped, but are implied. Similarly at node 2 we write

$$
\left\{\hat{\mathrm{u}}_{\mathrm{a}}^{2}\right\}=\left[\mathrm{T}_{1 \mathrm{e}}\right]\left\{\hat{\mathrm{u}}_{\mathrm{g}}^{2}\right\}
$$

Thus, for the element

$$
\left\{\begin{array}{c}
\left\{\hat{\mathrm{u}}_{\mathrm{a}}^{1}\right\} \\
\left\{\hat{\mathrm{u}}_{\mathrm{a}}^{2}\right\}
\end{array}\right\}=\left[\begin{array}{cc}
{\left[\mathrm{T}_{1 \mathrm{e}}\right]} & 0 \\
0 & {\left[\mathrm{~T}_{1 \mathrm{e}}\right.}
\end{array}\right]\left\{\begin{array}{c}
\left\{\hat{\mathrm{u}}_{\mathrm{g}}^{1}\right\} \\
\left\{\hat{\mathrm{u}}_{\mathrm{g}}^{2}\right\}
\end{array}\right\}
$$

or

$$
\left\{\hat{\mathrm{u}}_{\mathrm{a}}\right\}=\left[\mathrm{T}_{1}\right]\left\{\hat{\mathrm{u}}_{\mathrm{g}}\right\}
$$

in which $\left[\mathrm{T}_{1}\right]$ is a 14-by-14 matrix.

The generalized beam displacements are referenced to the local cartesian system $(x, y, z)$. Consider a global fixed cartesian system $(\overline{\mathrm{x}}, \overline{\mathrm{y}}, \overline{\mathrm{z}})$. Let the direction cosines between these two coordinate systems be denoted by $\langle x, \bar{x}\rangle,\langle x, \bar{y}\rangle, \ldots,\langle z, \bar{z}\rangle$. Then the generalized beam displacements in the local system at node 1 are related to the global system components by 


$$
\left\{\begin{array}{c}
\hat{U}_{1} \\
\hat{V}_{1} \\
\hat{W}_{1} \\
\hat{\Phi}_{\mathrm{x} 1} \\
\hat{\Phi}_{\mathrm{y} 1} \\
\hat{\Phi}_{\mathrm{z} 1} \\
\hat{\tau}_{1}
\end{array}\right\}=\left[\begin{array}{ccccccc}
\langle\mathrm{x}, \overline{\mathrm{x}}\rangle\langle\mathrm{x}, \overline{\mathrm{y}}\rangle\langle\mathrm{x}, \overline{\mathrm{z}}\rangle & 0 & 0 & 0 & 0 \\
\langle\mathrm{y}, \overline{\mathrm{x}}\rangle\langle\mathrm{y}, \overline{\mathrm{y}}\rangle\langle\mathrm{y}, \overline{\mathrm{z}}\rangle & 0 & 0 & 0 & 0 \\
\langle\mathrm{z}, \overline{\mathrm{x}}\rangle\langle\mathrm{z}, \overline{\mathrm{y}}\rangle\langle\mathrm{z}, \overline{\mathrm{z}}\rangle & 0 & 0 & 0 & 0 \\
0 & 0 & 0 & \langle\mathrm{x}, \overline{\mathrm{x}}\rangle & \langle\mathrm{x}, \overline{\mathrm{y}}\rangle\langle\mathrm{x}, \overline{\mathrm{z}}\rangle & 0 \\
0 & 0 & 0 & \langle\mathrm{y}, \overline{\mathrm{x}}\rangle\langle\mathrm{y}, \overline{\mathrm{y}}\rangle\langle\mathrm{y}, \overline{\mathrm{z}}\rangle & 0 \\
0 & 0 & 0 & \langle\mathrm{z}, \overline{\mathrm{x}}\rangle\langle\mathrm{z}, \overline{\mathrm{y}}\rangle & \langle\mathrm{z}, \overline{\mathrm{z}}\rangle & 0 \\
0 & 0 & 0 & 0 & 0 & 0 & 1
\end{array}\right]\left\{\begin{array}{c}
\overline{\mathrm{U}}_{1} \\
\overline{\mathrm{V}}_{1} \\
\overline{\mathrm{W}}_{1} \\
\bar{\Phi}_{\mathrm{x} 1} \\
\bar{\Phi}_{\mathrm{y} 1} \\
\bar{\Phi}_{\mathrm{z} 1} \\
\bar{\tau}_{1}
\end{array}\right\}
$$

or in matrix form

$$
\left\{\hat{\mathrm{u}}_{\mathrm{g}}^{1}\right\}=\left[\mathrm{T}_{\mathrm{gl}}\right]\left\{\overline{\mathrm{u}}_{1}\right\}
$$

in which the 7-by-7 matrix $\left[\mathrm{T}_{\mathrm{gg}}\right]$ is the global to local transformation matrix. In Eq. (4.52), the overbar on the nodal displacement designates that it is defined in the global cartesian direction. The warping degree of freedom $\tau$ in the local system is assumed to be the same in the global system, as is shown in the last equation of Eqs.(4.52). Since $\tau$ is proportional to the rate of twist about the local $\mathrm{x}$-axis, it does not transform as the rotation does.(assuming small rotations so that rotation can be represented as a vector). If two elements connected to the same node have discontinuous tangents, this assumption implies the rate of twist about the local $\mathrm{x}$-axis in each respective element is the same, since $\tau$ in the global system at the common node is the same as $\tau$ in the local systems of each element. If two adjacent elements have the same tangent at a common node, then $\tau$ is the same in each local coordinate system as one would expect. As before, this transformation is applied to each end of the element as follows. 


$$
\left\{\begin{array}{c}
\left\{\hat{u}_{\mathrm{g}}^{1}\right\} \\
\left\{\hat{\mathrm{u}}_{\mathrm{g}}^{2}\right\}
\end{array}\right\}=\left[\begin{array}{cc}
{\left[\mathrm{T}_{\mathrm{gl}}\right]} & 0 \\
0 & {\left[\mathrm{~T}_{\mathrm{g} 1}\right]}
\end{array}\right]\left\{\begin{array}{l}
\left\{\overline{\mathrm{u}}_{1}\right\} \\
\left\{\overline{\mathrm{u}}_{2}\right\}
\end{array}\right\}
$$

Call this transformation $\left[T_{2}\right]$. The total transformation from global cartesian to element coordinates can now be expressed as

$$
\left\{\hat{\mathrm{u}}_{\mathrm{a}}\right\}=\left[\mathrm{T}_{3}\right]\{\overline{\mathrm{u}}\}
$$

where $\left[T_{3}\right]=\left[T_{1}\right]\left[T_{2}\right]$, and in another form,

$$
\left[\mathrm{T}_{3}\right]=\left[\begin{array}{cc}
{\left[\mathrm{T}_{\mathrm{ge}}^{1}\right]} & {[0]} \\
{[0]} & {\left[\mathrm{T}_{\mathrm{ge}}^{2}\right]}
\end{array}\right]
$$

where

$$
\left[\mathrm{T}_{\mathrm{ge}}^{\mathrm{i}}\right]=\left[\begin{array}{ccccccc}
\langle\mathrm{x}, \overline{\mathrm{x}}\rangle & \langle\mathrm{x}, \overline{\mathrm{y}}\rangle & \langle\mathrm{x}, \overline{\mathrm{z}}\rangle & 0 & 0 & 0 & 0 \\
\langle\mathrm{y}, \overline{\mathrm{x}}\rangle & \langle\mathrm{y}, \overline{\mathrm{y}}\rangle & \langle\mathrm{y}, \overline{\mathrm{z}}\rangle & 0 & 0 & 0 & 0 \\
0 & 0 & 0 & \langle\mathrm{z}, \overline{\mathrm{x}}\rangle & \langle\mathrm{z}, \overline{\mathrm{y}}\rangle & \langle\mathrm{z}, \overline{\mathrm{z}}\rangle & 0 \\
\langle\mathrm{z}, \overline{\mathrm{x}}\rangle & \langle\mathrm{z}, \overline{\mathrm{y}}\rangle & \langle\mathrm{z}, \overline{\mathrm{z}}\rangle & 0 & 0 & 0 & 0 \\
\frac{-\langle\mathrm{x}, \overline{\mathrm{x}}\rangle}{\left(\mathrm{a}-\mathrm{z}_{\mathrm{R}}\right)} & \frac{-\langle\mathrm{x}, \overline{\mathrm{y}}\rangle}{\left(\mathrm{a}-\mathrm{z}_{\mathrm{R}}\right)} & \frac{-\langle\mathrm{x}, \overline{\mathrm{z}}\rangle}{\left(\mathrm{a}-\mathrm{z}_{\mathrm{R}}\right)} & -\langle\mathrm{y}, \overline{\mathrm{x}}\rangle & -\langle\mathrm{y}, \overline{\mathrm{y}}\rangle & -\langle\mathrm{y}, \overline{\mathrm{z}}\rangle & 0 \\
0 & 0 & 0 & \langle\mathrm{x}, \overline{\mathrm{x}}\rangle & \langle\mathrm{x}, \overline{\mathrm{y}}\rangle & \langle\mathrm{x}, \overline{\mathrm{z}}\rangle & 0 \\
0 & 0 & 0 & \frac{\langle\mathrm{z}, \overline{\mathrm{x}}\rangle}{\left(\mathrm{a}-\mathrm{z}_{\mathrm{R}}\right)} & \frac{\langle\mathrm{z}, \overline{\mathrm{y}}\rangle}{\left(\mathrm{a}-\mathrm{z}_{\mathrm{R}}\right)} & \frac{\langle\mathrm{z}, \overline{\mathrm{z}}\rangle}{\left(\mathrm{a}-\mathrm{z}_{\mathrm{R}}\right)} & 1
\end{array}\right]
$$

As a result of element curvature, the tangent at each end of the element will be in different directions in the global cartesian system. In general, $\left[\mathrm{T}_{\mathrm{ge}}^{1}\right] \neq\left[\mathrm{T}_{\mathrm{ge}}^{2}\right]$. Substituting 
Eq.(4.55), into $\delta \mathrm{W}_{\mathrm{int}}=\delta \hat{\mathrm{u}}_{\mathrm{a}}^{\mathrm{T}}\left[\mathrm{K}_{\mathrm{r}}^{\mathrm{e}}\right] \hat{\mathrm{u}}_{\mathrm{a}}$, gives $\delta \mathrm{W}_{\text {int }}=\delta \mathrm{u}^{-\mathrm{T}}\left[\mathrm{K}^{\mathrm{g}}\right] \overline{\mathrm{u}}$, where the reduced element stiffness matrix in the global cartesian coordinate system is

$$
\left[K^{g}\right]=\left[T_{3}\right]^{T}\left[K_{r}^{e}\right]\left[T_{3}\right]
$$

\subsection{CALCULATION OF DIRECTION COSINES}

For calculation of the direction cosines needed in $\left[\mathrm{T}_{3}\right]$, an orientation node is added to each element description. There are no element degrees of freedom associated with this node. The orientation node in connection with the two end nodes determines the plane of curvature and the concave direction of the beam within that plane. In the present theory, straight beams are approximated using a large radius of curvature, so in this case, the only effect of the nodes location is to orient the cross-section.

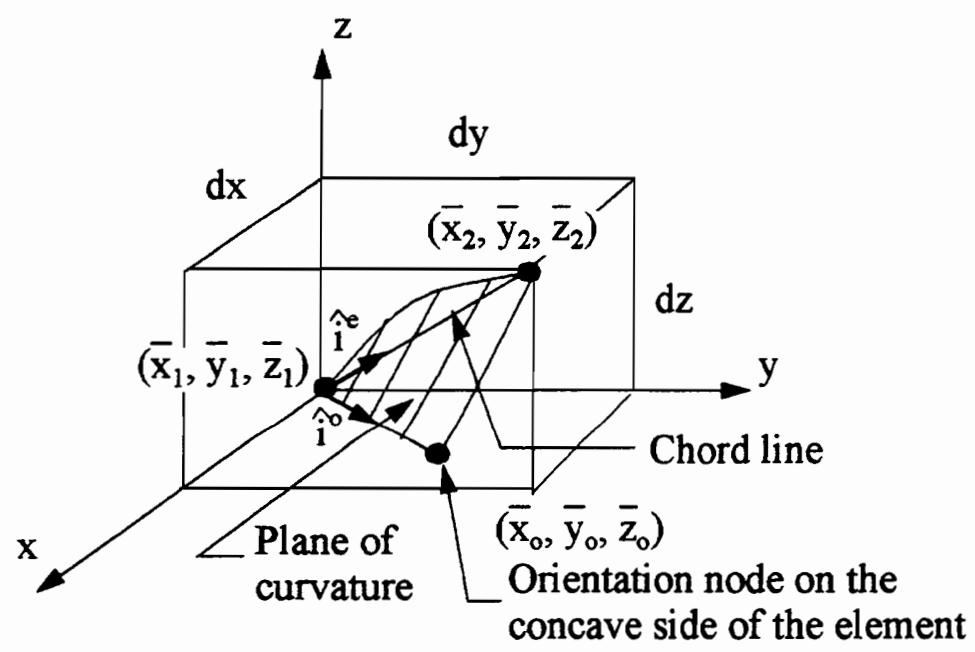

Figure 4.3 Element description

The element chord line is described by a unit vector in the direction from node one, to node two.

$$
\hat{\imath}^{e}=\frac{\Delta x}{1_{e}} \hat{\imath}+\frac{\Delta y}{1_{e}} \hat{\jmath}+\frac{\Delta z}{T_{e}} \hat{K}
$$


where

$$
\begin{aligned}
& \Delta x=x_{2}-x_{1} \\
& \Delta y=y_{2}-y_{1} \\
& \Delta z=z_{2}-z_{1} \\
& 1_{e}=\sqrt{\Delta x^{2}+\Delta y^{2}+\Delta z^{2}}
\end{aligned}
$$

The orientation vector is a unit vector along the line from node one to the orientation node.

$$
\hat{\mathbf{l}}^{\mathrm{o}}=\frac{\Delta \mathrm{x}_{\mathrm{o}}}{\mathrm{l}_{0}} \hat{\mathrm{i}}+\frac{\Delta \mathrm{y}_{\mathrm{o}}}{1_{0}} \hat{\mathrm{j}}+\frac{\Delta \mathrm{z}_{\mathrm{o}}}{\mathrm{l}_{0}} \hat{\mathrm{K}}
$$

where

$$
\begin{aligned}
& \Delta x_{0}=x_{0}-x_{1} \\
& \Delta y_{0}=y_{0}-y_{1} \\
& \Delta z_{0}=z_{0}-z_{1} \\
& 1_{0}=\sqrt{\Delta x_{0}^{2}+\Delta y_{0}^{2}+\Delta z_{0}^{2}}
\end{aligned}
$$

The unit vector $\hat{j}^{e}$, along the element $y$-axis is now determined as the cross product $\hat{1}^{e} \times \hat{1}^{o}$, and the unit vector $\mathrm{k}^{\mathrm{e}}$ along the element $\mathrm{z}$-axis is determined from the cross product $\hat{i}^{e} \times \hat{j}^{e}$. The transformation from the global system to the $\left(\hat{i}^{e}, \hat{j}^{e}, \hat{k}\right)$ system is written as

$$
\left\{\begin{array}{c}
\hat{\mathbf{i}}^{\mathrm{e}} \\
\hat{\mathrm{j}}^{\mathrm{e}} \\
\hat{\mathrm{K}}^{\mathrm{e}}
\end{array}\right\}=\left[\begin{array}{lll}
1_{11} & 1_{12} & 1_{13} \\
l_{21} & 1_{22} & 1_{23} \\
1_{31} & 1_{32} & 1_{33}
\end{array}\right]\left\{\begin{array}{l}
\hat{\mathbf{I}} \\
\hat{\mathbf{J}} \\
\hat{\mathrm{K}}
\end{array}\right\}=[1]\left\{\begin{array}{c}
\hat{\mathbf{I}} \\
\hat{\mathbf{J}} \\
\hat{\mathrm{K}}
\end{array}\right\}
$$

At each end of the element, an additional rotation around the element y-axis must be per- 
formed to rotate from the element chord line to the element tangent direction.

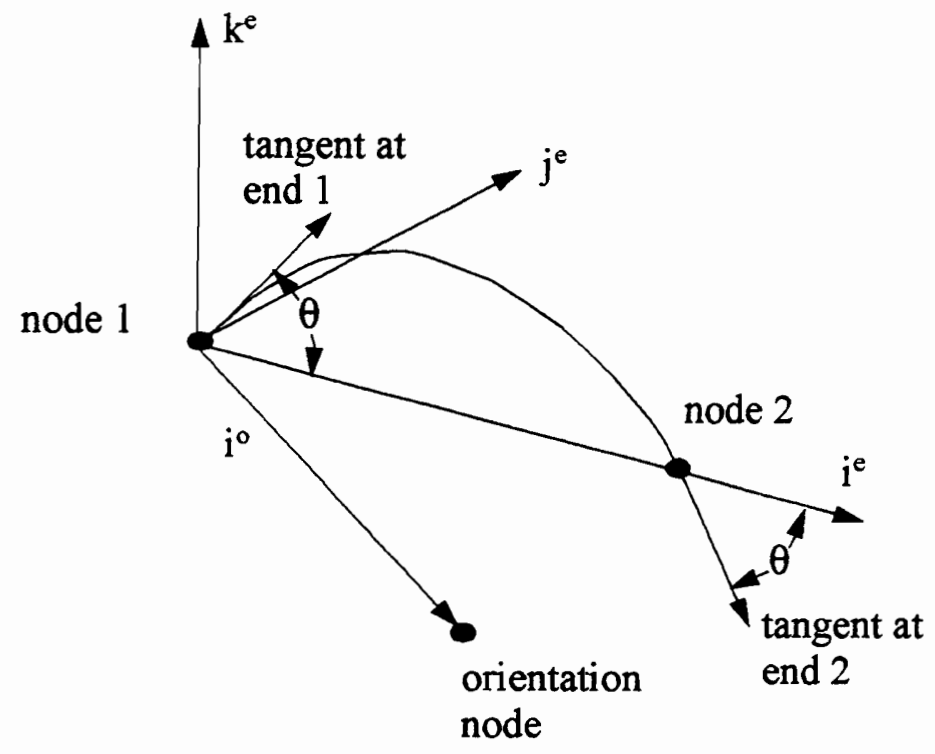

Figure 4.4 Element end rotation

The angle for this rotation at each end is given as

$$
\theta=\arcsin \left(\frac{1_{e}}{2 \mathrm{R}}\right)
$$

where $\mathrm{R}$ is the radius of curvature for the element. Thus the rotation from $\left(\hat{i}^{\mathrm{e}}, \dot{\hat{j}}^{\mathrm{e}}, \mathrm{k}^{\mathrm{e}}\right)$, to the local system $(\hat{\mathrm{i}}, \hat{\mathrm{j}}, \hat{\mathrm{K}})$, is

$$
\left\{\begin{array}{c}
\hat{\imath} \\
\hat{\jmath} \\
\mathrm{k}
\end{array}\right\}=\left[\mathrm{T}_{\theta}\right]\left\{\begin{array}{c}
\hat{\imath}^{\mathrm{i}} \\
\hat{j}^{\mathrm{e}} \\
\hat{k}^{\mathrm{e}}
\end{array}\right\}
$$

where. 


$$
\left[T_{\theta}\right]=\left[\begin{array}{ccc}
\cos (\theta) & 0 & \sin (\theta) \\
0 & 1 & 0 \\
-\sin (\theta) & 0 & \cos (\theta)
\end{array}\right]
$$

Note that $\theta$ is positive at node one, and negative at node two. The total transformation from global to local systems is

$$
\left\{\begin{array}{c}
\hat{\imath} \\
\hat{\jmath} \\
\hat{k}
\end{array}\right\}=\left[\mathrm{T}_{\theta}\right][1]\left\{\begin{array}{c}
\hat{\mathrm{I}} \\
\hat{\jmath} \\
\hat{\mathrm{K}}
\end{array}\right\}
$$

so that the direction cosines in Eq.(4.57) are computed by

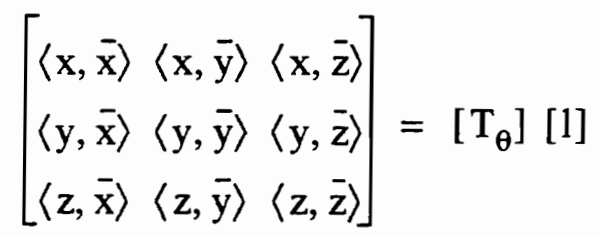




\section{CHAPTER 5}

\section{ANALYSIS VERIFICATION}

\subsection{INTRODUCTION}

The curved thin-walled composite beam theory presented in Chapter 2 is evaluated by a comparison of the finite element beam solution with equivalent results from other sources. There are two objectives for this evaluation: 1.) to insure that the finite element model is a valid implementation of the theory, and 2.) to explore the accuracy and limitations of the current composite beam theory.

\section{2 FINITE ELEMENT EVALUATION}

For the first objective, the finite element results are compared to a series of analytical solutions, and to data reported in the literature. General analytical solutions for the thin-walled warping beam theory were available for isotropic straight beams, and for isotropic curved beams with symmetric cross sections under radial loads only. For the configurations examined, the finite element results matched the analytical solutions with a high degree of precision as is shown below. Several examples from these comparisons are presented here as a demonstration of the performance of the finite element and its characteristics.

The first example problem for consideration is the arch shown in Figure 5.1. This arch 
configuration was used by Dawe ${ }^{[5]}$ for numerical studies of arch finite elements.

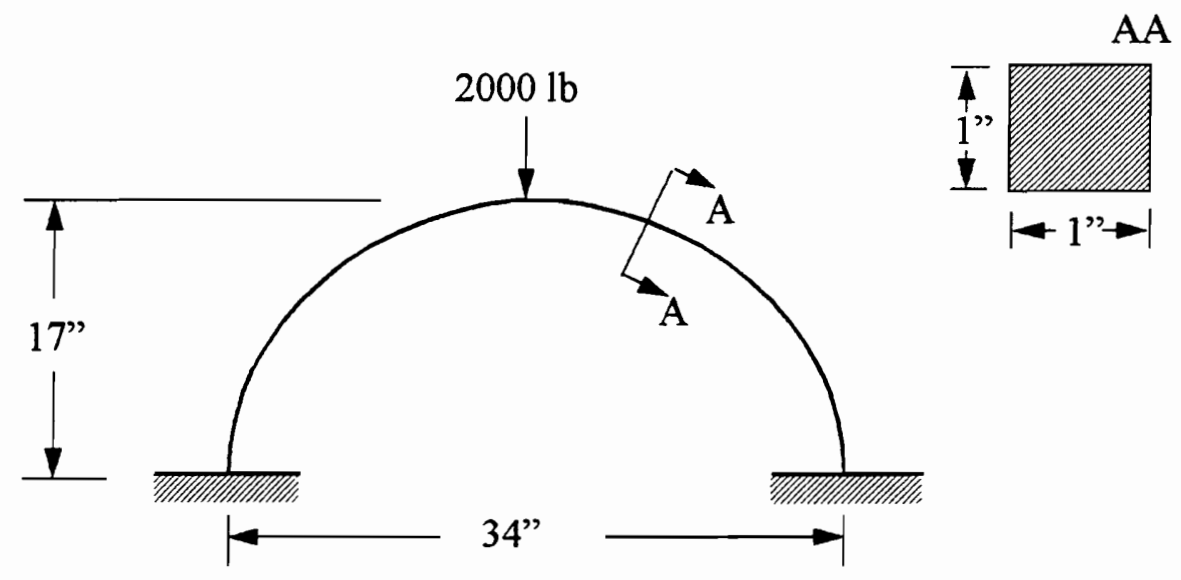

Figure 5.1 Circular arch under a central load

As shown in Table 5.1, the current element exhibits excellent convergence characteristics.

Note that the finite element gives the exact analytic solution for this case ( for a BernoulliEuler formulation of a circular arch with bending and extension).

Table 5.1 Deflection at the applied load from the finite element model of the arch shown in Figure 5.1

\begin{tabular}{cccc}
\hline \hline $\begin{array}{c}\text { Number of } \\
\text { Elements for the } \\
\text { half arch }\end{array}$ & $\begin{array}{c}\text { Number of } \\
\text { degrees of } \\
\text { freedom }\end{array}$ & $\begin{array}{c}\text { Center } \\
\text { deflection } \\
\text { (inches) }\end{array}$ & $\begin{array}{c}\text { Error } \\
(\%)\end{array}$ \\
\hline 1 & 1 & 0.14142135 & 0.07239 \\
2 & 4 & 0.14152314 & 0.00046 \\
3 & 7 & 0.14152380 & 0.00000 \\
\hline
\end{tabular}

${ }^{a}$ Error with respect to exact center deflection $=0.1415238$ inches.

A second example demonstrates the accuracy of the implementation under torsional loads, as well as the principal advantage of the present beam theory over simpler elements based on St. Venant theory for torsion. This advantage is improved accuracy for torsional load cases where cross-sectional warping is restrained. This effect is demonstrated using the straight beam configuration shown in Figure 5.2. Torsional analysis was performed with 
and without warping restraint at the ends of the beam for the two different cross sections, and for three beam lengths (see Figure 5.2.).
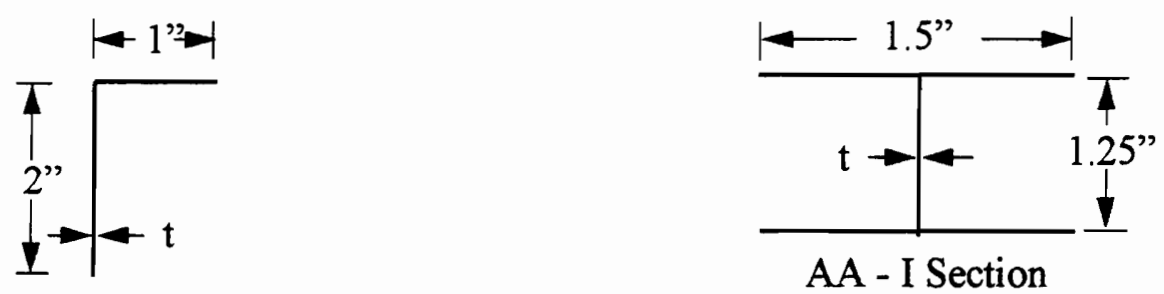

\section{AA - Angle Section}

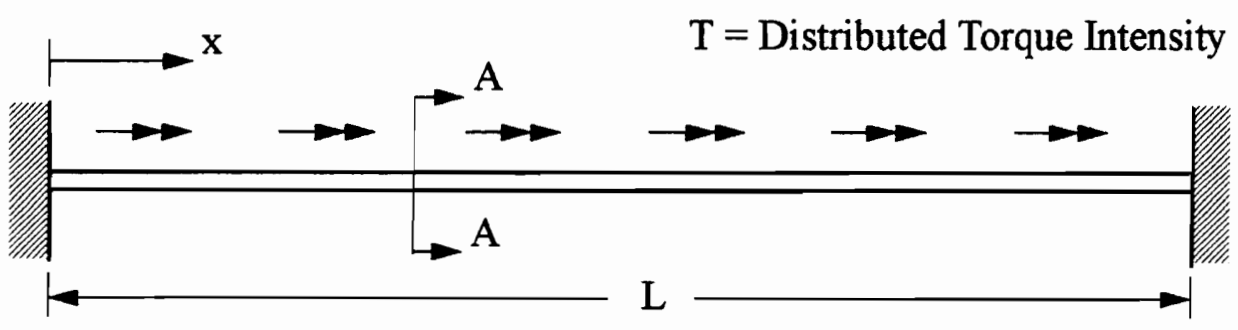

Figure 5.2 Straight beam test geometry

In these examples, the beam material is isotropic and homogeneous with a modulus of elasticity $E=10.5 * 10^{6}$, Poissons ration $v=0.33$, and the wall thickness $t$ is .042 in., and the beam is subjected to a uniformly distributed torque of $1.0 \mathrm{lb} / \mathrm{in}$. For convenience, twenty curved beam elements were used along the length of the beam.

Results for the angle section are given in Table 5.2, and results for the I section are given in Table 5.3. The "warping restrained" column refers to an analysis which does not allow the additional axial warping displacements at the fixed ends of the beam. For a straight 
beam, the "warping free" column is equivalent to St. Venant torsion theory.

Table 5.2 Finite element performance for a straight isotropic angle section subjected to a distributed torsion

\begin{tabular}{cccccc}
\hline \hline & & \multicolumn{2}{c}{ Angle of Rotation $\Phi \mathrm{x}(\mathrm{x}=\mathrm{L} / 2)$ Radians } & \\
\cline { 3 - 5 } $\begin{array}{c}\mathrm{L} \\
\text { (inches) }\end{array}$ & $\mu_{1}$ & Analytical $^{\mathrm{a}}$ & $\begin{array}{c}\text { F.E.M. } \\
\text { Warping } \\
\text { restrained }\end{array}$ & $\begin{array}{c}\text { F.E.M. } \\
\text { Warping } \\
\text { Free }\end{array}$ & $\begin{array}{c}\% \Delta \\
\text { Free .vs. } \\
\text { restrained }\end{array}$ \\
\hline 100 & 110.30 & 4.2292 & 4.2284 & 4.370 & 3.34 \\
50 & 55.15 & 1.0231 & 1.0233 & 1.090 & 6.50 \\
25 & 27.58 & 0.2387 & 0.2387 & 0.271 & 13.5 \\
\hline \hline
\end{tabular}

${ }^{\text {a From Gjelsvik }}{ }^{[46]}$ p.139

Table 5.3 Finite element performance for a straight isotropic I section subjected to a distributed torsion.

\begin{tabular}{cccccc}
\hline \hline & & \multicolumn{2}{c}{ Angle of Rotation $\Phi \times(x=L / 2)$ Radians } & \\
\cline { 3 - 5 } \begin{tabular}{c} 
L \\
\cline { 3 - 5 }
\end{tabular} & $\mu_{1}$ & Analytical $^{\mathrm{L}}$ & $\begin{array}{c}\text { F.E.M. } \\
\text { Warping } \\
\text { restrained }\end{array}$ & $\begin{array}{c}\text { F.E.M. } \\
\text { Warping } \\
\text { Free }\end{array}$ & $\begin{array}{c}\% \Delta \\
\text { Free .vs. } \\
\text { restrained }\end{array}$ \\
\hline 100 & 6.73 & 1.3570 & 1.3800 & 2.5900 & 87.6 \\
50 & 3.37 & 0.1402 & 0.1460 & 0.4310 & 195.2 \\
25 & 1.68 & 0.0105 & 0.0113 & 0.0464 & 310.6 \\
\hline \hline
\end{tabular}

${ }^{\text {a }}$ From Gjelsvik ${ }^{[46]}$ p. 139.

For torsion of straight beams, Gjelsvik ${ }^{[46}$ gives a dimensionless parameter $\mu_{1}=\mathrm{L} / \sqrt{\left(\mathrm{EI}_{\omega \omega} / \mathrm{GJ}\right)}$ that relates the ratio of St. Venant $T_{\mathrm{SR}}$, to the warping torque $\mathrm{T}_{\omega}$. (The total torque $\mathrm{T}=\mathrm{T}_{\mathrm{SR}}+\mathrm{T}_{\omega}$, where $\mathrm{T}_{\mathrm{SR}}=\mathrm{GJ} \Phi_{\mathrm{xR}}^{\prime}, \mathrm{T}_{\omega}=-\mathrm{M}_{\omega}=-\mathrm{EI}_{\omega \omega} \Phi_{\mathrm{xR}}^{\prime \prime \prime}$, and primes denote derivatives with respect to longitudinal coordinate $\mathrm{x}$.). When warping is restrained, the warping $T_{\omega}$ often dominates St. Venant $T_{S R} \cdot{ }^{1}$ The ratio of $T_{\omega} / T_{S R}$ is gener-

1. For free warping, $T_{\omega}=0$ and the $S t$. Venant torque is the total torque. 
ally large when $\mu_{1}$ is small, and in this case Vlasov torsion is a good approximation to mixed torsion. When $\mu_{1}$ is large St. Venant torsion generally dominates except in localized zones (boundary layers) near discontinuities where warping is restrained. Thus, when $\mu_{1}$ is large restrained warping has generally less effect on torsional stiffness.

This restrained warping effect is illustrated by the results given in Table 5.2, and in Table 5.3. For the angle section, where $\mu_{1}$ is relatively large, the difference in torsional stiffness due to warping varies from 3.3 to 13.5 percent, while for the I section which has a smaller $\mu_{1}$, the difference ranges from 87.6 to 310.6 percent. These examples demonstrate the potential for error that exists when a warping theory is not used for torsional loads of thin walled beams. Finite element results presented in these tables are in close agreement with the analytical predictions for these straight beam configurations.

Because analytical solutions for all possible configurations are not available, the first objective of establishing a correct finite element implementation of the theory, cannot be satisfied with $100 \%$ certainty. In particular, the curved beam cases with anisotropic wall construction, under torsional or out of plane loads, present a problem since no analytical solutions were found.

\subsection{COMPARISON TO BRANCHED SHELL ANALYSIS}

The second objective of the evaluation process was to establish the accuracy and limitations of the current theory. Since thin-walled laminated curved composite beams are often analyzed using the branched shell method, results from the current theory were evaluated by comparison to results from an equivalent branched shell finite element analysis. For this evaluation, the branched shell analysis was conducted using the $\mathrm{EAL}^{\left[{ }^{57}\right]}$ computer code, with membrane and bending quad laminated plate elements ${ }^{1}$. Convergence studies were performed for both the beam models, and the branched shell models. These branched

1. EAL element designation E43. 
shell results provide a benchmark against which the current theory can be judged.

Given the large number of possible combinations of beam geometries, curvatures, boundary conditions, load cases, cross section geometries, laminate constructions, and material types, it is necessary to select a limited set of cases for evaluation which will serve as indicators of the performance of this theory under more general conditions. To establish a basis for selecting these cases, several important differences between the branched shell theory and the curved beam theory are considered. First, the branched shell theory allows for in-plane distortion of the cross section, and in-plane shear strain of the middle surface, while these deformations are precluded by assumptions 1 and 2 of the current curved beam theory. Second, the branched shell solution captures some of the material coupling behavior which is lost in the transformation given by Eq. (2.101). Discrepancies in the results should be traceable to one or more of these theoretical differences. With this in mind, a restricted set of evaluation cases was assembled to demonstrate the accuracy and limitations in the current theory. The test matrix contains four basic cross-sectional geometries; a blade section oriented in the plane of curvature, an angle section, an I section, and a channel section. These represent two symmetric cross sections, and two unsymmetric cross sections, where one of each type is predicted by Gjelsvik's warping parameter $\mu_{1}$, to be more sensitive to warping restraint than the other (assuming $\mu_{1}$ is representative of curved beams). The ratio of flange length to flange thickness (b/t) for these sections is relatively large. It is likely that a large (b/t) ratio will permit distortion of the cross section to 
influence the results if it is a factor. These cross sections are illustrated in Figure 5.3

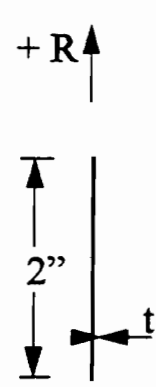

Blade
Symmetric

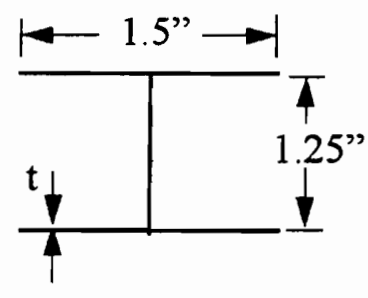

I

Unsymmetric

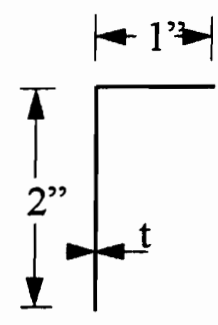

Angle

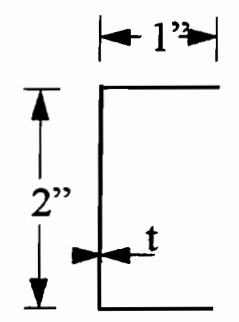

Channel

Wall thickness $t$ is the same for each branch

Figure 5.3 Beam cross sections for EAL comparisons

For each of these cross section geometries, two materials were used to create six wall constructions;

1.) Aluminum ( $\left.E=10.5^{*} 10^{6} \mathrm{psi}, \mathrm{v}=.33, \mathrm{t}=.042^{\prime \prime}\right)$

2.) AS4/5208, graphite epoxy $\left(\mathrm{E}_{1}=18.4 \times 10^{6} \mathrm{psi}, \mathrm{E}_{2}=1.64 \times 10^{6} \mathrm{psi}, \mathrm{G}_{12}=.87 \times 10^{6} \mathrm{psi}\right.$, $\left.v_{12}=.30, t=.00525^{\prime \prime}\right)$, with five stacking sequences.
a.) $[+45 /-45 / 0 / 90]_{s}$
b.) $\left[+45_{4} /-45_{4}\right]_{\mathrm{T}}$
c.) $\left[0_{4} / 90_{4}\right]_{T}$
d.) $\left[+30_{4} /-30_{4}\right]_{T}$
e.) $\left[+30_{8}\right]_{\mathrm{T}}$

Note that some of these are unusual laminates specially selected to excite a particular material coupling mode, and are not expected to be encountered in practice. Gjelsvik's parameter on a per unit length basis is recorded in Table 5.4, for each cross section / mate- 
rial combination.

Table 5.4 Gjelsvik's parameter per unit length $\mu_{1} / L$ for the curved beam evaluation cases (I/in)

\begin{tabular}{ccccc}
\hline \hline & \multicolumn{2}{c}{ Symmetric } & \multicolumn{2}{c}{ Unsymmetric } \\
\cline { 2 - 5 } & Blade & $\mathrm{I}$ & Angle & Channel \\
\hline Iso & 2.12500 & 0.06738 & 1.10300 & 0.10050 \\
Quasi & 3.18800 & 0.08157 & 1.58600 & 0.12170 \\
{$\left[+45_{4} /-45_{4}\right]_{\mathrm{T}}$} & 3.49800 & 0.10210 & 1.79200 & 0.15380 \\
{$\left[0_{4} / 90_{4}\right]_{\mathrm{T}}$} & 1.47900 & 0.03234 & 0.71810 & 0.04852 \\
{$\left[+30_{4} /-30_{4}\right]_{\mathrm{T}}$} & 2.65200 & 0.06845 & 1.32200 & 0.10210 \\
{$\left[+30_{8}\right]_{\mathrm{T}}$} & 2.03100 & 0.09185 & 1.10200 & 0.13680 \\
\hline \hline
\end{tabular}

Results for each cross section / material combination were calculated using three different beam configurations; a straight case, and two different curved cases. Each beam was analyzed for three load cases; a radial load, a torsional load, and an out of plane load (transverse). These loads were all applied at mid-span, and each beam was fixed for all degrees of freedom (including warping), at each end. The basic test geometry is shown in Figure 5.4 .

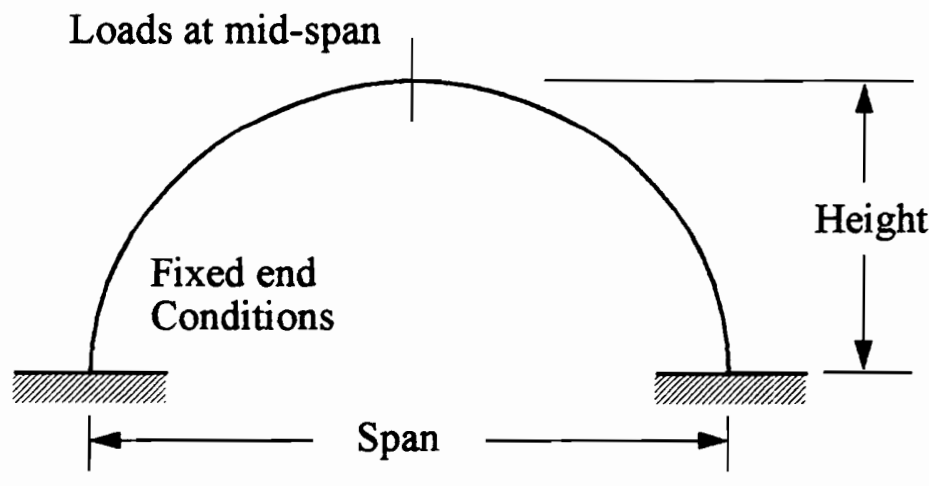

Figure 5.4 Curved beam test Geometry

The analytical results from a selection of these comparisons are presented in Figure 5.5 through Figure 5.10. The bar graphs in these figures represent a ratio of the beam displace- 
ment from the present beam theory, to the branched shell displacement calculated using E.A.L. The displacements are taken at the point of load application, in the direction of the applied load, and so represent the compliance of the structure under that load. A value of 1.0 indicates perfect agreement between the two theories. The curved beam case is a 36 inch radius of curvature circular arch (Span $=72 "$, Height $\left.=36^{\prime \prime}\right)$. Note that "b/t", refers to the ratio of the length of the widest free flange in the cross section (b) to it's thickness $(t)$.

Straight Blade

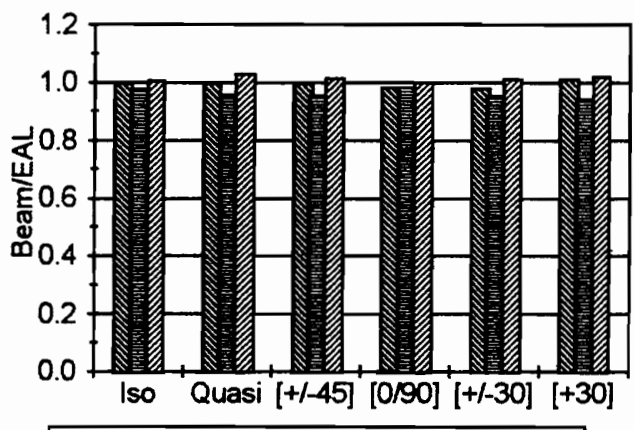

MRadial 酎Torsion שTansverse

\section{Curved Blade R=36"}

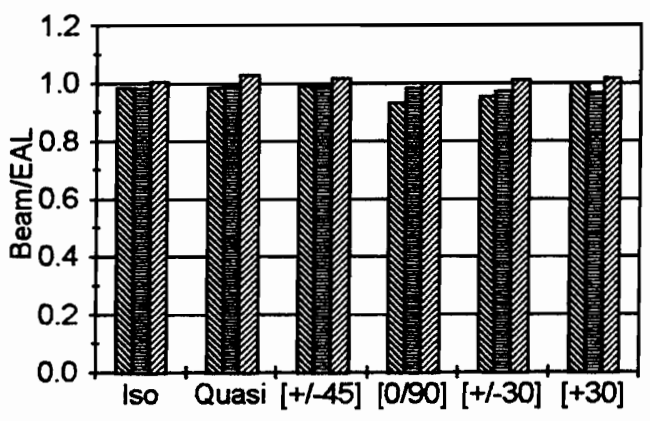

MRadial Torsion Transverse

Figure 5.5 Blade section compliance ratios $(b / t=17.85)$
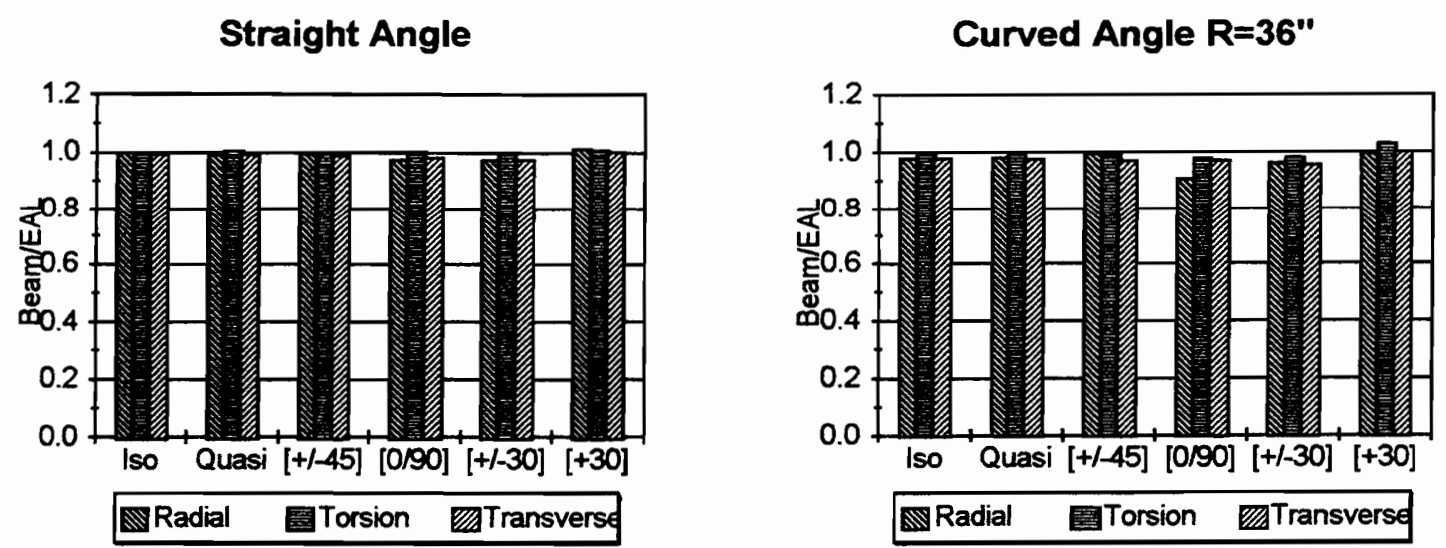

Figure 5.6 Angle section compliance ratios $(b / t=17.85)$ 


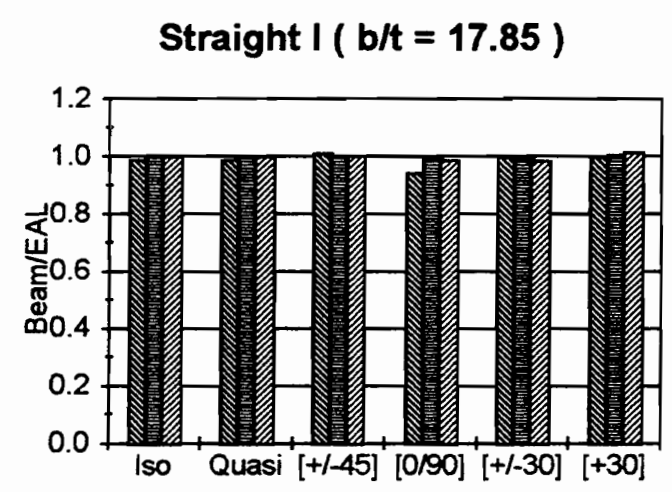

$\mathbb{N}$ Radial :

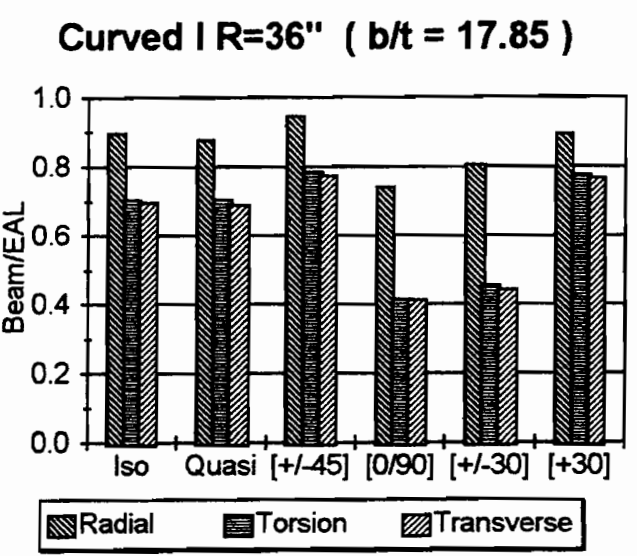

Figure $5.7 I$ - section compliance ratios $(b / t=17.85)$

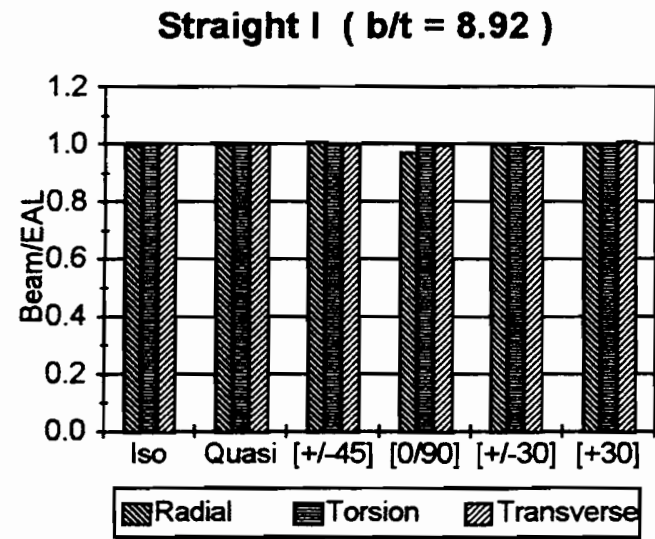

Curved I R=36" ( $b / t=8.92$ )

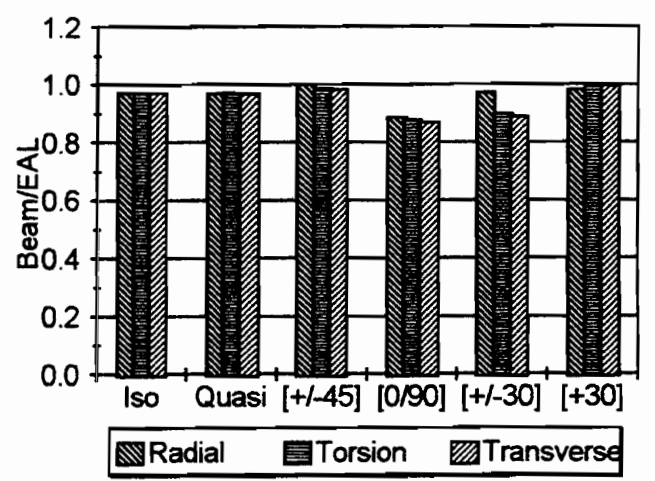

Figure $5.8 I$ - section compliance ratios $(b / t=8.92)$

\section{Straight Channel ( $b / t=17.85$ )}

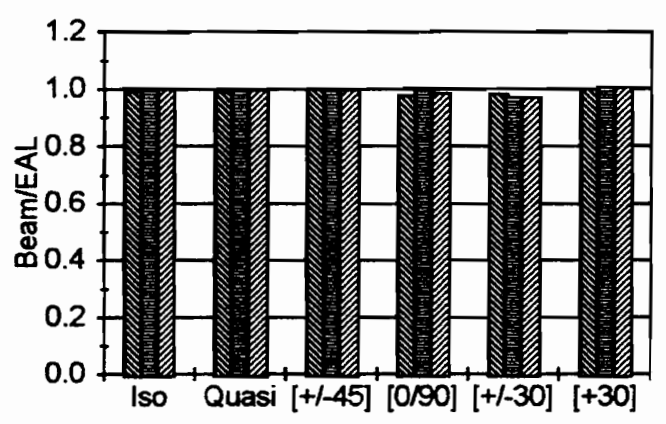

WRadial 国Torsion WTransverse
Curved Channel $R=36$ " ( $b / t=17.85$ )

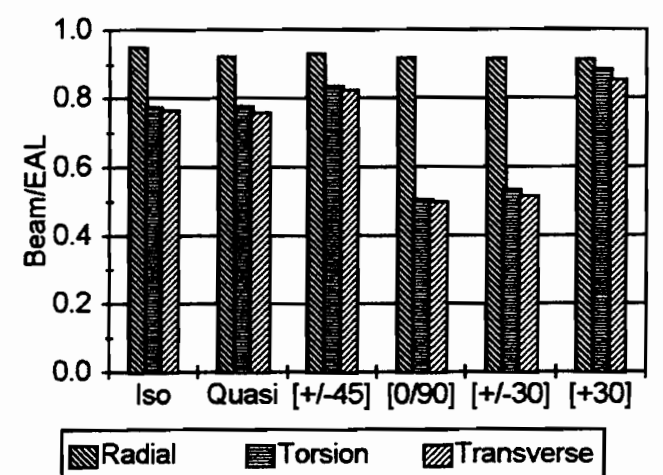

Figure 5.9 Channel section compliance ratios $(b / t=17.85)$ 


\section{Straight Channel $(b / t=11.9)$}

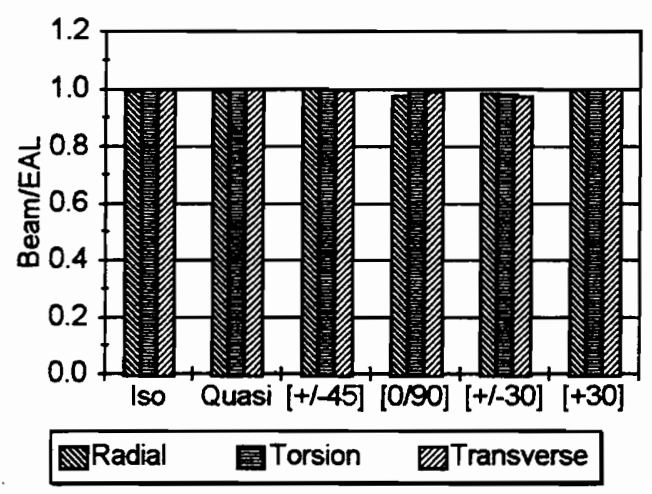

Curved Channel R=36" ( $b / t=11.9$ )

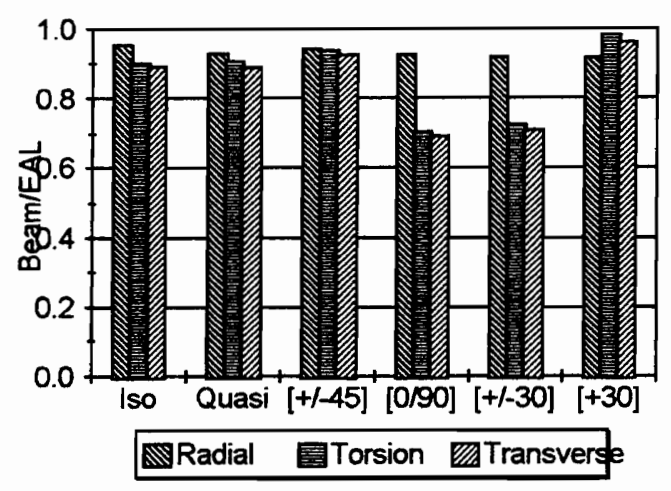

Figure 5.10 Channel section compliance ratios $(b / t=11.9)$

As shown in Figure 5.5 through Figure 5.10, excellent agreement is obtained for the straight beams. Displacement results for all cross sections, materials, and load cases match closely. Given the unusual laminates chosen for comparison, these results are interesting. This seems to indicate that the important material coupling behavior is captured by the current theory, and the assumptions made in Eq. (2.101) are appropriate. Note that for all of these analysis, the value used for $\delta$ in Eq. (2.101) was set to 1.0, corresponding with the assumption of vanishing reactive resultants. Values of $\delta$ less than 1.0 resulted in excessive stiffness. This observation is in agreement with the findings of $\mathrm{Wu}$, and Sun ${ }^{[52]}$.

For the curved beams, the situation is somewhat more complicated. Results from these comparisons demonstrate a dependence of the accuracy of the beam theory on cross-sectional geometry, radius of curvature, stacking sequence, and loading. The dependance of accuracy on cross section geometry is conspicuous. Analytical results from the curved blade and angle cross section beam models are in close agreement with the branched shell theory for all load cases and stacking sequences, even for relatively large ratio's of $b / t$ ( see Figure 5.5, and Figure 5.6). Contrast this with results from the curved I section and curved channel section analysis. For these cross-sectional geometries, analysis results from current beam theory are too stiff. Agreement is generally better for the radial load 
case, and the isotropic and quasi-isotropic stacking sequences, and improves dramatically as $b / t$ is reduced (See Figure 5.8 and Figure 5.10). The conclusion from these analytical comparisons is that curvature, and cross-sectional geometry will have the most significant influence on accuracy of the current composite curved beam theory, with laminate stacking sequence and load configuration acting as secondary factors.

\subsubsection{INFLUENCE OF IN-PLANE DISTORTION OF THE CROSS SECTION}

The dependence of accuracy on cross-sectional geometry and curvature is partially explained by distortion of the cross section in its plane. Work by Mabson ${ }^{[58]}$, and Peck ${ }^{[59}$ ], examined this phenomenon with respect to the effects of composite beam construction. To understand how distortion affects the accuracy of curved beam analysis, consider a segment of curved beam with an I cross section under pure bending as shown in Figure 5.11 .
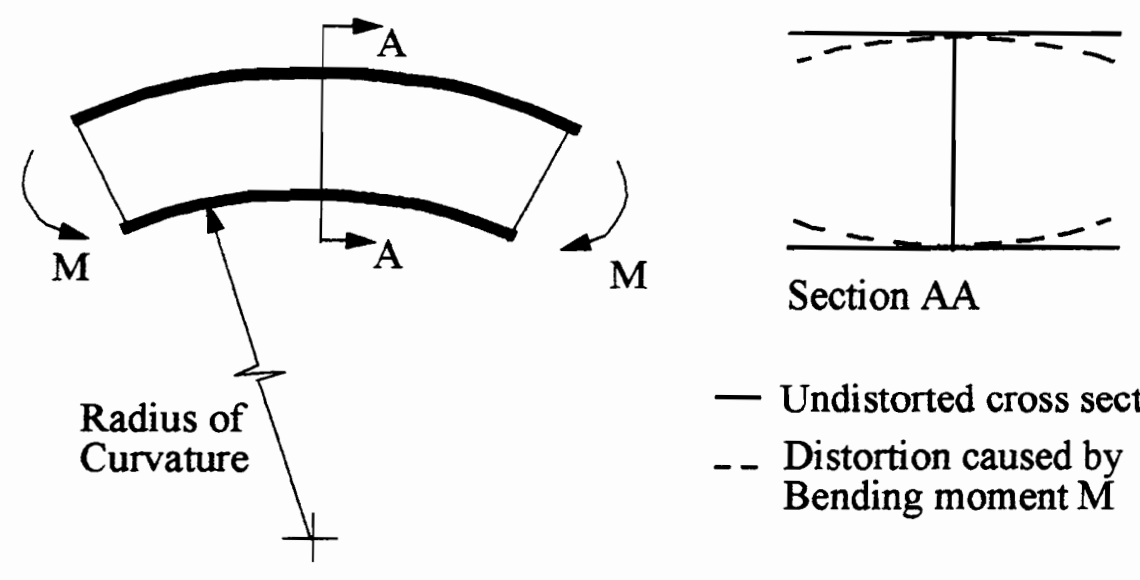

- Undistorted cross section

- - Distortion caused by Bending moment $M$

\section{Figure 5.11 Cross section distortion}

Because of the coupling which exists between circumferential deformations and radial deformations in a curved shell, the ends of the horizontal flanges of the curved beam cross section move radially. The distorted shape of the cross section shown in Figure 5.11 represents a lower energy equilibrium configuration of the curved beam. Circumferential strain 
is reduced as the flange moves radially. Peck ${ }^{[59]}$ demonstrates that for a curved I-section, this reduction in circumferential strain due to distortion can be large enough to result in a change from compression at the root to a small tension at the flange tips. Relieving strain at the flange tips, reduces the effective flange length, since more of the load is now carried by the center portion of the cross-section. This reduces the effective moment of inertia and makes the distorted beam appear more flexible, which is consistent with the errors observed in the present beam theory. If the moment were reversed, the distortion would reverse, but the increase in flexibility will be similar.

As the flange aspect ratio $(\mathrm{b} / \mathrm{t})$ is decreased, the ratio of flange transverse bending stiffness to flange length is increased, and there is less tendency for the distortion to occur. As the radius of curvature is reduced, the geometric shell coupling becomes stronger and the tendency for distortion is increased. These trends are consistent with the discrepancies encountered in the comparison of beam results to branched shell analysis. Although the distortion effect is illustrated using a pure bending load on an I section, it will be observed with any circumferential load regardless of its origin, on any curved beam cross section flange that is oriented appropriately and free to bend. Thus, the circumferential stresses that are caused by warping and transverse loading will also result in cross section distortion. The warping case is illustrated for an I section in Figure 5.11

I cross section of curved beam

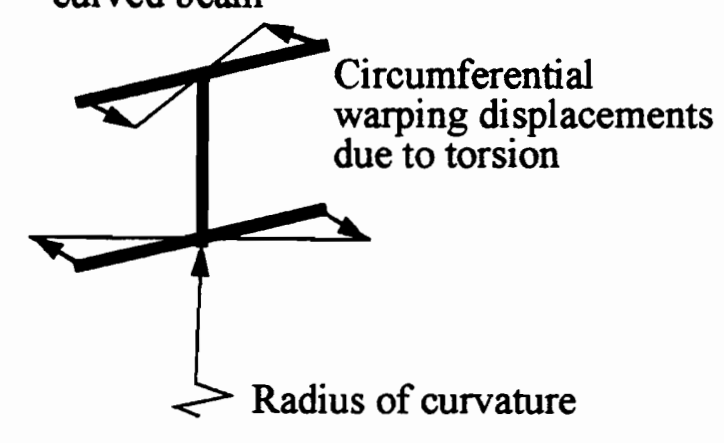

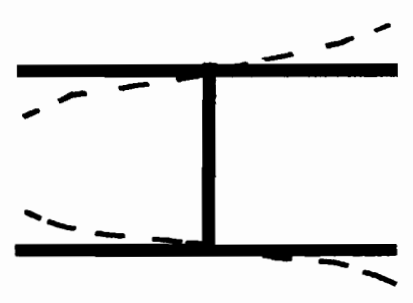

- Undistorted cross section

- - Distortion caused by torsion

Figure 5.12 Torsional related distortion for curved beams. 
Cross section distortions in the branched shell model are caused by the distribution of circumferential forces along the curved beam flanges. These flange loads vary with angular position along the beam, causing the distortion to vary in a similar fashion. The total change in response is the net effect of distortions along the span. The existence of cross section distortions in the branched shell models is verified by an examination of the shell strains and displacements. The transverse bending strain $\left(K_{s S}\right)$ becomes non-zero for the curved beam flanges and the curvature patterns match those shown in Figure 5.11 and Figure 5.12. Cross section distortions are not allowed by the kinematic assumptions of the current beam theory. It is believed that cross section distortions are the primary cause of inaccuracies observed in the current beam theory.

\subsubsection{INFLUENCE OF LAMINATE STACKING SEQUENCE AND LOADING}

Cross-sectional geometry and beam curvature are the primary factors influencing cross section distortion, but accuracy of the current theory was also observed to be dependent on laminate stacking sequence and loading. Accuracy is generally lower for the transverse and torsional load cases, and in particular for the $\left[0_{4} / 90_{4}\right]$ and the $\left[+30_{4} /-30_{4}\right]$ stacking sequences. An examination of the branched shell strains indicates that the curvatures ( $\kappa_{\theta \theta}$, $\kappa_{S S}$, and $\kappa_{\theta S}$ ) are significantly and consistently higher for the transverse and torsional load cases. Since $\kappa_{s s}$ is a measure of cross-sectional distortion, it appears that increased levels of distortion are present for the torsional and transverse load cases. In addition, the magnitude of the shear strain $\gamma_{\theta s}$, is approaching the magnitude of circumferential strain $\varepsilon_{\theta \theta}$ for the $\left[0_{4} / 90_{4}\right],\left[+30_{4} /-30_{4}\right]$, and $\left[+30_{8}\right]$ stacking sequences. Since the curved beam theory assumes that strains $\boldsymbol{\kappa}_{\mathrm{ss}}$, and $\gamma_{\theta \mathrm{s}}$, are zero along the curved beam contour, it is reasonable to conclude that increases in these strains and curvatures are either directly or indirectly responsible for the reduced accuracy of these load cases and laminates.

The connection between cross-sectional distortion (increased $\mathrm{K}_{\mathrm{ss}}$ ) and torsion is the shell coupling between circumferential strains, and radial displacements. As the cross section 
rotates, circumferential strain is altered as the flange tips move radially. The flanges will distort to relieve strain at the tips. This effect is not equivalent to the one described previously. In this case a radial displacement leads to a circumferential strain, where before, a circumferential strain caused by the bimoment led to a radial displacement. Recalling that torsional and transverse displacements are coupled by geometry, this additional distortion effect may be sufficient to explain the lower accuracy observed for these load cases. Several important observed trends are consistent with this explanation; 1.) The accuracy is only affected for torsional and transverse load cases, 2.) Accuracy decreases as curvature increases, and 3.) Reducing flange aspect ratio (b/t) improves the results.

The lower accuracy for the $\left[0_{4} / 90_{4}\right]$ and the $\left[+30_{4} /-30_{4}\right]$ stacking sequences, is believed to be related to the relative increase in shear strain. To investigate this assumption, it is instructive to look at the laminate stiffness matrices for the laminates used in these beam comparisons.

Table 5.5 Extensional stiffness matrix $[A], l b /$ in

\begin{tabular}{ccccccc}
\hline \hline & $\mathrm{A}_{11}$ & $\mathrm{~A}_{12}$ & $\mathrm{~A}_{16}$ & $\mathrm{~A}_{22}$ & $\mathrm{~A}_{26}$ & $\mathrm{~A}_{66}$ \\
\hline Iso & $341.6 \mathrm{e} 3$ & $103.4 \mathrm{e} 3$ & 0 & $341.6 \mathrm{e} 3$ & 0 & $119.1 \mathrm{e} 3$ \\
Quasi & $494.9 \mathrm{e} 3$ & $163.3 \mathrm{e} 3$ & 0 & $494.9 \mathrm{e} 3$ & 0 & $165.7 \mathrm{e} 3$ \\
{$\left[+45_{4} /-45_{4}\right]_{\mathrm{T}}$} & $259.1 \mathrm{e} 3$ & $185.9 \mathrm{e} 3$ & 0 & $259.1 \mathrm{e} 3$ & 0 & $201.7 \mathrm{e} 3$ \\
{$\left[0_{4} / 90_{4}\right]_{\mathrm{T}}$} & $424.2 \mathrm{e} 3$ & $20.83 \mathrm{e} 3$ & 0 & $424.2 \mathrm{e} 3$ & 0 & $36.54 \mathrm{e} 3$ \\
{$\left[+30_{4} /-30_{4}\right]_{\mathrm{T}}$} & $477.7 \mathrm{e} 3$ & $144.7 \mathrm{e} 3$ & 0 & $122.9 \mathrm{e} 3$ & 0 & $160.4 \mathrm{e} 3$ \\
{$\left[+30_{8}\right]_{\mathrm{T}}$} & $477.7 \mathrm{e} 3$ & $144.7 \mathrm{e} 3$ & $225.2 \mathrm{e} 3$ & $122.9 \mathrm{e} 3$ & $82.1 \mathrm{e} 3$ & $160.4 \mathrm{e} 3$ \\
\hline \hline
\end{tabular}

Table 5.6 Coupling stiffness matrix $[B], l b / i n$

\begin{tabular}{ccccccc}
\hline \hline & $\mathrm{B}_{11}$ & $\mathrm{~B}_{12}$ & $\mathrm{~B}_{16}$ & $\mathrm{~B}_{22}$ & $\mathrm{~B}_{26}$ & $\mathrm{~B}_{66}$ \\
\hline Iso & 0 & 0 & 0 & 0 & 0 & 0 \\
Quasi & 0 & 0 & 0 & 0 & 0 & 0 \\
{$\left[+45_{4} /-45_{4}\right]_{\mathrm{T}}$} & 0 & 0 & -1862.7 & 0 & -1862.7 & 0 \\
{$\left[0_{4} / 90_{4}\right]_{\mathrm{T}}$} & -3725.0 & 0 & 0 & 3725.0 & 0 & 0 \\
{$\left[+30_{4} /-30_{4}\right]_{\mathrm{T}}$} & 0 & 0 & -2364.0 & 0 & -862.2 & 0 \\
{$\left[+30_{8}\right]_{\mathrm{T}}$} & 0 & 0 & 0 & 0 & 0 & 0 \\
\hline \hline
\end{tabular}


Table 5.7 Bending stiffness matrix [D] lb/in

\begin{tabular}{ccccccc}
\hline \hline & $\mathrm{D}_{11}$ & $\mathrm{D}_{12}$ & $\mathrm{D}_{16}$ & $\mathrm{D}_{22}$ & $\mathrm{D}_{26}$ & $\mathrm{D}_{66}$ \\
\hline Iso & 46.0 & 24.3 & 7.33 & 36.72 & 7.33 & 26.61 \\
Quasi & 72.74 & 24.0 & 0 & 72.74 & 0 & 24.37 \\
{$\left[+45_{4} /-45_{4}\right]_{\mathrm{T}}$} & 38.08 & 27.34 & 0 & 38.08 & 0 & 29.65 \\
{$\left[0_{4} / 90_{4}\right]_{\mathrm{T}}$} & 62.36 & 3.06 & 0 & 62.36 & 0 & 5.37 \\
{$\left[+30_{4} /-30_{4}\right]_{\mathrm{T}}$} & 70.23 & 21.27 & 0 & 18.07 & 0 & 23.58 \\
{$\left[+30_{8}\right]_{\mathrm{T}}$} & 70.23 & 21.27 & 33.09 & 18.07 & 12.07 & 23.58 \\
\hline \hline
\end{tabular}

The presence of shear strain in a laminate can affect the results directly, and also indirectly through material coupling. Notice that the $\left[0_{4} / 90_{4}\right]$ laminate has the lowest shear stiffness $\mathrm{A}_{66}$, when compared to the other laminates. This laminate is much more likely to deform in shear, which is not allowed by assumptions of the current beam theory. A check of the shell model strains confirms that the shear strains in the flanges are on the order of the circumferential strains for this stacking sequence. This observation is consistent with the lower accuracy for this analysis case.

Next observe that the $\left[+30_{4} /-30_{4}\right]$ laminate has non-zero $\mathrm{B}_{16}$ and $\mathrm{B}_{26}$ terms. These terms couple shear to bending, and twist to extension. This will affect the accuracy since coupling between shear and transverse bending $\left(B_{26}\right)$ can exaggerate the problem of crosssectional distortion. The $\left[+45_{4} /-45_{4}\right]$ laminate also has these non-zero $B_{16}$ and $B_{26}$ terms, but results for beams using this laminate are consistently more accurate. A possible explanation for this apparent inconsistency is that for this laminate, the ratio of axial stiffness to shear stiffness is very low (1.28), compared to the equivalent ratio (2.97) for the [ $+30_{4}$ $\left.30_{4}\right]$ laminate. Thus the $\left[+45_{4} /-45_{4}\right]$ laminate is less likely to deform by shear and more likely to deform axially.

Further evidence for this coupling effect can be found by comparing the laminate stiffness matrix entries of the $\left[+30_{4} /-30_{4}\right]$ laminate, to the $\left[+30_{8}\right]$ laminate. The $\left[+30_{8}\right]$ laminate has non-zero $A_{16}, A_{26}, D_{16}$, and $D_{26}$ terms, and the $B_{16}$ and $B_{26}$ terms are zero, otherwise the 
matrix entries are identical to the $\left[+30_{4} /-30_{4}\right]$ laminate. Analysis results for beams using this $\left[+30_{8}\right]$ laminate are consistently and significantly more accurate. It appears that material couplings from the $A_{16}, A_{26}, D_{16}$, and $D_{26}$ terms are either captured by the beam theory, or are not significant. Levels of shear strain are similar for both stacking sequences. The differences in accuracy observed for these two cases support the premise that the $\mathrm{B}_{26}$ coupling of shear to distortion is one source of the problem. From this discussion of material behavior, we can conclude that the observed variation in accuracy for the same cross section constructed from different laminates is caused by the assumption of zero shear strain in the beam theory.

Gjelsvik's parameter $\mu_{1} / L$ as given for these beams in Table 5.4, quantifies one of the fundamental differences between the cross section types. For the channel and I sections, $\mu_{1} / L$ is relatively small compared to values obtained for the blade and angle. This parameter can be viewed as an indicator of how torsional energy will be stored in the beam ( Bimoment .vs. Saint Venant Torque). For the channel and I sections, significantly more torque energy is stored as a bimoment when compared to the blade and angle. Recall that the bimoment causes circumferential strains which in turn lead to cross section distortions. The blade and the angle, which have relatively low warping stiffness, are less prone to cross section distortion and analysis errors. The change in $\mu_{1} / \mathrm{L}$ with respect to $\mathrm{b} / \mathrm{t}$ is given for the curved I and channel sections in Table 5.8.

Table 5.8 Values $\mu_{1} / L$ for different $b / t$ ratios

\begin{tabular}{ccccc}
\hline \hline & \multicolumn{2}{c}{$\mathrm{I}$} & \multicolumn{2}{c}{ Channel } \\
& $\mathrm{b} / \mathrm{t}=17.85$ & $\mathrm{~b} / \mathrm{t}=8.92$ & $\mathrm{~b} / \mathrm{t}=17.85$ & $\mathrm{~b} / \mathrm{t}=11.9$ \\
\hline Iso & 0.06738 & 0.15280 & 0.10050 & 0.15730 \\
Quasi & 0.08157 & 0.18500 & 0.12170 & 0.19060 \\
{$[+45 /-45]$} & 0.10310 & 0.23390 & 0.15380 & 0.24090 \\
{$[0 / 90]$} & 0.03234 & 0.07336 & 0.04852 & 0.07544 \\
{$[+30 /-30]$} & 0.68450 & 0.15530 & 0.10210 & 0.15990 \\
{$[+30]$} & 0.09185 & 0.20820 & 0.13680 & 0.21420 \\
\hline \hline
\end{tabular}


The curved beam results for torsion, and transverse loads of Figure 5.7, and Figure 5.9, appear discouraging, however there are two factors to consider. First, the alternative beam theory, namely a Bernoulli-Euler, or Timoshenko beam with Saint Venant torsion, was found to be in error by several hundred percent (excessive flexibility) for these same torsional and transverse load problems ( see Table 5.9).

Table 5.9 Comparison of compliance ratios ("Beam Theory" / EAL branched shell) for the isotropic curved I section ( $\left.R=36^{\prime \prime}, b / t=17.85\right)$

\begin{tabular}{cccc}
\hline \hline & Radial & Torsional & Transverse \\
\hline Current Theory & 0.89380 & 0.70253 & 0.69724 \\
Bernoulli-Euler & 0.89750 & 3.34100 & 3.56800 \\
Timoshenko & 0.96330 & 3.30800 & 3.34580 \\
\hline \hline
\end{tabular}

Second, these results were obtained with a considerable reduction in computational effort. The typical beam model from the current theory would require 28 degrees of freedom to obtain the results presented here, while the branched shell models required between 6 and 15 thousand degrees of freedom, depending on the cross section (see Table 5.10)

Table 5.10 Degrees of freedom used for E.A.L.shell analysis

\begin{tabular}{cc}
\hline Cross-Section & $\begin{array}{c}\text { Degrees of } \\
\text { freedom in shell } \\
\text { model }\end{array}$ \\
\hline I- Section & $15,678.0$ \\
Channel & $10,854.0$ \\
Angle & $8,442.0$ \\
Blade & 6030.0 \\
\hline
\end{tabular}

The comparisons done in this Chapter revealed that it is important to use the curved-beam form of the contour warping function $\bar{\omega}(\mathrm{s})$ Eq.(2.44) versus the straight-beam form Eq.(2.45). Although it may seem reasonable to use the simpler straight form for thinwalled beams, calculations show that this results in a dependence of the torsional response on the reference point location. This dependence is not correct, and disappears when the 
curved-beam form of the contour warping function is used.

One objective of this analysis verification was to establish limitations for the current theory. The following guidelines are recommended based on the theory and observations discussed in this chapter.

1.) The radius of curvature should be large compared to the basic dimensions of the cross section.

2.) High aspect ratio flanges $(b / t)$ should be used with caution if $\mu_{1} / L$, and the radius of curvature are relatively small, especially if torsional or transverse loads are present.

3.) Stacking sequences with low shear stiffness $A_{66}$, and/or large $B_{26}$ should be used with caution if $\mu_{1} / L$, and the radius of curvature are relatively small, especially if torsional or transverse loads are present. 


\section{CHAPTER 6}

\section{PROGRESSIVE FAILURE ANALYSIS}

\section{1 INTRODUCTION}

Progressive failure analysis is an attempt to predict the load-response of a structure beyond the initial failure event, and conceivably up to the point of catastrophic or total failure. Failure events are defined as any significant change in strength or stiffness. These changes are frequently caused by localized material yielding or failure, local or global instabilities, and changes in contact or friction. The structural model for progressive failure analysis must adapt to reflect these changes as the analysis progresses.

Dynamic non-linear finite element crash analysis codes such as DYNA3D ${ }^{[25]}$ and DYCAST ${ }^{[26]}$ implement the adaptive process of progressive failure primarily through non-linear material laws, some of which allow for damage and failure. For these programs, instability failures must be initiated through small geometric imperfections in the nodal mesh, or through eccentrically applied loads. Contact algorithms are included, but are computationally very expensive. Efficient modeling requires prior assumptions about which surfaces are likely to make contact. These analysis methods have been used successfully for progressive failure analysis of metallic structures, but success with composites has been limited. This is largely due to the difficulty associated with predicting and modeling composite failure and damage. Composite failure modes are dependent on load- 
ing, and are sensitive to random defects. Work is ongoing to improve progressive failure analysis of composite structures using detailed nonlinear finite element methods ${ }^{[24]}$, but further research is required. As discussed in Chapter 1, these analytical progressive analysis methods are considered to be too expensive computationally for practical optimization in todays computing environment.

The other main class of crash finite element analysis programs ( e.g. KRASH ${ }^{[27]}$ ) does not predict the failure behavior of structural components, but instead relies on user input of nonlinear load-response data. The disadvantage of this method is that component progressive failure behavior must be established in advance. The dynamic structural loads and accelerations are obtained from this method, but otherwise it does not help to characterize the load-response behavior of individual structural elements. Since a requirement of progressive failure analysis in the present work is to characterize the response of a structural component so that it can later be improved using optimization, this class of programs is not directly applicable.

Incremental finite element analysis is another approach to progressive failure. With this method, the load (or displacement) is incremented in steps. At each load step, the elements are updated, a stiffness matrix is formed, and the equations are solved. The approach taken by Miles ${ }^{[29]}$ is to use an incremental stiffness matrix, and an incremental displacement vector at each step. A geometric transformation is applied to each element as part of the update. Thin walled beam elements are modified to account for material non-linearity and pin-joints or spring hinges are added when the plastic moment is exceeded. This method was successfully used by Miles for the collapse analysis of thin-walled metallic frame structures. Mahmood and Paluszny ${ }^{[32]}$ propose a similar methodology with the program VCRUSH. In this approach, progressive failure of an element is divided into four phases; elastic, post-buckling, and deep collapse. The response of a beam element is modified to reflect its current phase by using "effective section properties" in the post-buckling phase, 
and "crush-type hinges" in the deep collapse region. Another example of the incremental approach is the finite element formulation by Toi and Yang ${ }^{[30}$. In Toi's work, a reduced integration technique is used to simulate the formation of plastic hinges in Timoshenko beam elements. The plastic hinge location can be located precisely within the element by shifting the integration point. These incremental methods are significantly more efficient than the dynamic ( time dependent )finite element methods, because they use efficient structural elements, and rely on pre-determined failure models to modify the element stiffness matrices. The progressive failure analysis developed as part of this work can be considered a variation of this basic incremental analysis approach

\section{2 PROGRESSIVE FAILURE ANALYSIS}

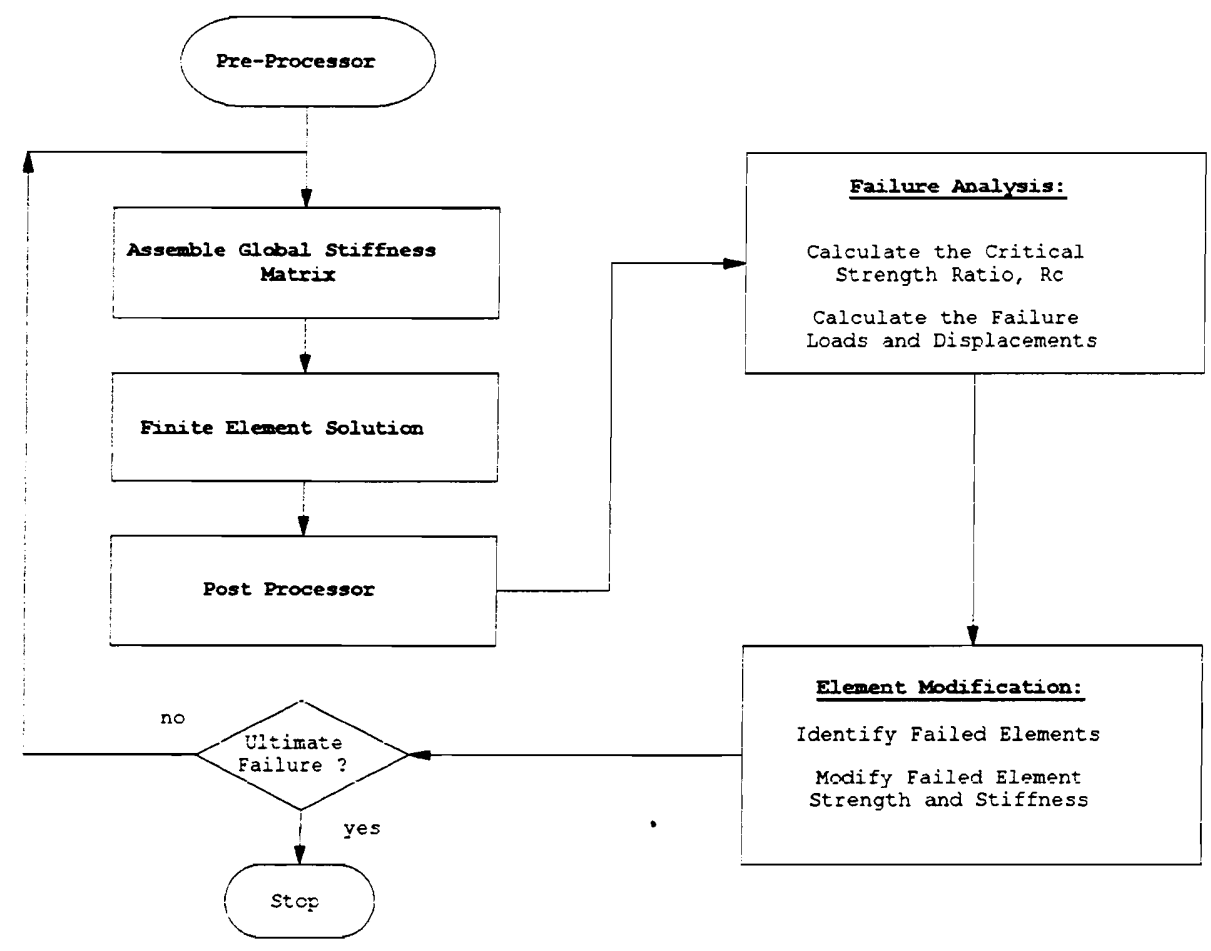

Figure 6.1 Computational diagram of progressive failure analysis

A progressive failure analysis using the present laminated composite curved beam theory 
was developed and implemented as part of this work. This method was developed specifically for progressive failure analysis of composite fuselage frames. The characteristic brittle failure mode exhibited by these frames allows us to use a series of linear solutions to approximate the progressive failure solution. Finite element analysis is used to predict the element strains, and a progressive failure model is used to predict changes in strength and stiffness. The cycle of structural analysis followed by failure analysis and structural modification is repeated until ultimate failure occurs. The flow diagram for this procedure is shown in Figure 6.1

Approximation of the progressive failure solution is accomplished in a step-wise manner. Each cycle of analysis identifies one or more critical failure events, which in turn determine the failure loads and displacements. Failures are required to be discrete and brittle, so that response of the structure will remain linear, but will have a reduced strength and stiffness after failure. This requirement precludes the use of materials predisposed to fail by continuous plastic yield, and geometric configurations like short columns which fail by quasi-continuous crushing. It does not preclude buckling or local crippling which can be approximated by discrete changes in element stiffness. Because stiffnesses are changed by failure, a discreet load drop will occur at each failure displacement, and the load response will continue from that point with a modified stiffness. Analysis of the modified structure generates another failure point on the load response curve, and begins another step in the progressive failure solution. Figure 6.2 illustrates how the load response curve is gener- 
ated using this approach.

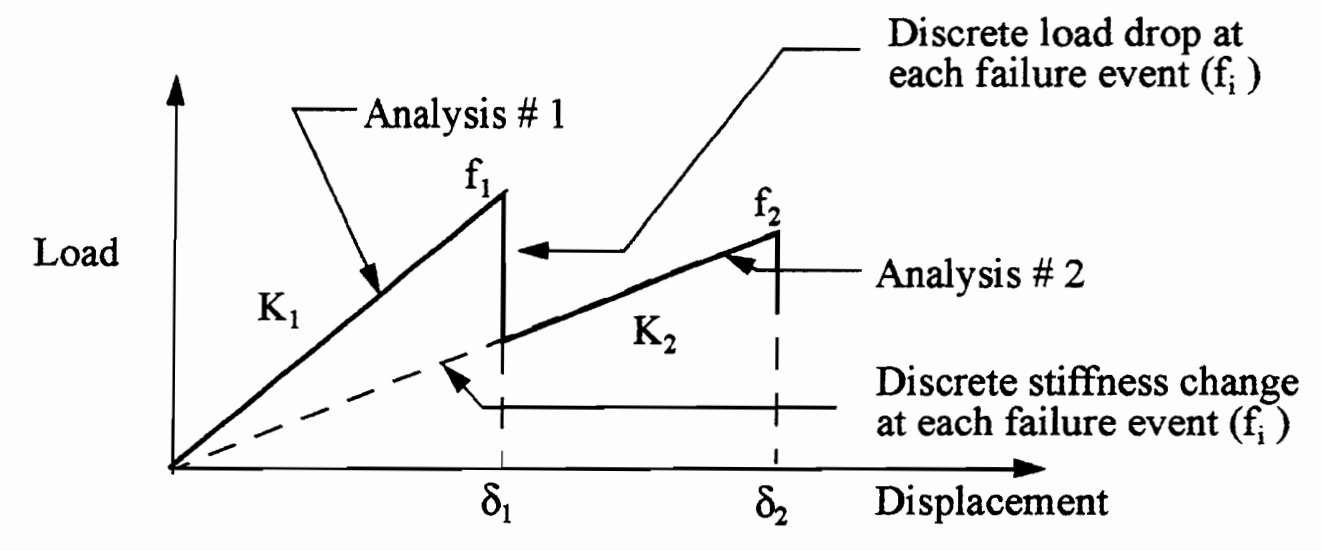

Figure 6.2 Progressive failure sequence

A key feature of progressive failure analysis is the failure model. The failure model determines which failure events will be captured by the analysis, and how the structure is modified in response to each event. The underlying mechanism for structural failure in the present work, is a progressive failure model for laminated composite materials. Failure models for contact, local crippling and buckling appear feasible, but are not implemented at this time. The laminate failure criteria and progressive failure model will be discussed in detail in a later section.

The physical mechanism for beam failure is composite material failure and degradation, but implementation of the material degradation in the beam model is complicated by the fact that the state of stress and strain is spatially non-uniform. The strains and failure margins of a beam element will in general vary along the length, and also over the cross section. Recall from Chapters 2 and 3, that the thin walled beam cross sections are modelled as an assembly of cross section branches. This is a discretization of the cross section. For progressive failure analysis, each section element contains a laminate that is free to fail progressively in an independent manner. Strains will in general vary between section elements, and also over the length, width, and thickness of each individual section element. One cross section element might be in tension, while another is in compression, or one end 
of an cross section element might be in tension, while the other end is in compression, etc. Here, the material failure model is applied at both ends of every section branch. The results are compared, and the critical margin is stored for later consideration. Another form of discretization is used along the length of each beam element. The cross section failure analysis is applied at six equally spaced locations along the length of each beam element. Section branches are continuous along the beam element length, so the critical margin from each cross-sectional slice is used for material failure and degradation of that branch. Degradation of one or more section branches will cause a change in cross section properties for that beam element. By selectively and independently modifying cross section branches in response to progressive material failures, this beam failure model progressively reduces and modifies cross-sectional stiffness as failure proceeds.

A consequence of this beam failure model is that the discretization of the cross section into branches can affect the failure sequence. When the number of independent elements in the cross section is increased, the failure sequence will be modeled more accurately. The cost of improving accuracy by increasing the number of cross section elements is a significant increase in computational requirements. Element post processing, and laminate failure analysis comprise a significant portion of the total solution time for each analysis step.

Failure analysis and structural modification are separate and sequential analysis steps. A displacement of arbitrary magnitude is applied to the load point on the structure to begin a structural finite element analysis. After analysis, the failure model is applied to each beam element in sequence, and strength ratios are calculated for every lamina.(Strength ratio is the ratio of allowable load to applied load.) The purpose of this failure analysis loop is to determine the minimum strength ratio. The minimum strength ratio is critical, and values less than one indicate failure. This critical ratio determines the magnitude of the actual failure displacement. Only after the critical strength ratio has been established, can the process of structural modification begin. This requires another loop over the beam ele- 
ments to modify the critical lamina. For composite thin walled beams, it often occurs that many cross section elements will be critical or near critical simultaneously. To limit the number of distinct failure events, a failure envelope was established. All section elements having a strength ratio within 2.5 percent of the minimum are identified as critical, and failed as a group.

If the calculated failure displacement increases from the previous analysis step, this becomes a new point on the load displacement curve of the progressive failure analysis. If the failure displacement for the modified structure is less than it was for the previous analysis, additional failures are allowed to occur until either the failure displacement increases, or ultimate failure occurs. The process is then repeated until ultimate failure occurs.

\subsection{MATERIAL FAILURE}

Material failure was identified as the principal mechanism for progressive structural failure in this analysis. To implement this choice, it is necessary to select a laminated composite failure theory from among the many that are available. A review that covers 21 failure theories for anisotropic materials is given by Rowlands $\left.{ }^{60}\right]$. This review stops short of recommending a specific failure theory, but singles out the failure theories of Puppo and Evensen $^{[61]}$, and Tsai-Wu ${ }^{[62]}$. For the present work, the material failure theory should account for progressive degradation of strength and stiffness for each lamina. Later versions of the theory by Tsai ${ }^{[39]}$ have this capability. Another advantage of using the Tsai$\mathrm{Wu}$ theory is that it contains other polynomial theories as special cases. Tsai's progressive failure theory for composite laminates is considered the most reasonable choice for use in the current progressive failure analysis.

The Tsai-Wu failure criterion for composite laminates is based on an extension of the Von Mises criterion for yielding of ductile metals to brittle failure of orthotropic materials. 
This is an empirical scheme to fit experimental data, and is not derived from fundamental principles. Tsai-Wu postulates that in stress space, the failure criterion can be represented as a quadratic form.

$$
F_{i j} \sigma_{i} \sigma_{j}+F_{i} \sigma_{i}=1, \text { for } i, j=1 . .6
$$

For an orthotropic material under plane stress, with stresses acting on faces normal to the material axes 1 and 2, Tsai-Wu's quadratic criterion is given by,

$$
\mathrm{F}_{11} \sigma_{1}^{2}+2 \mathrm{~F}_{12} \sigma_{1} \sigma_{2}+\mathrm{F}_{22} \sigma_{2}^{2}+\mathrm{F}_{66} \sigma_{6}^{2}+\mathrm{F}_{1} \sigma_{1}+\mathrm{F}_{2} \sigma_{2}=1
$$

The strength parameters " $F_{i j}$ " and " $F_{i}$ " are determined such that the failure envelope given by Eq.(6.2) passes through a set of material failure points established by simple uniaxial tension, compression and pure shear tests. Five basic material strengths are required to determine $F_{11}, F_{22}, F_{1}, F_{2}$, and $F_{66}$,

$\mathrm{X}$ - Tensile strength in the fiber direction.

$\mathrm{X}^{\prime}$ - Compressive strength in the fiber direction.

$\mathrm{Y}$ - Tensile strength in the transverse direction.

$\mathrm{Y}^{\prime}$ - Compressive strength in the transverse direction.

S - In-plane longitudinal shear strength

The axial strength parameters are obtained by setting $\sigma_{1}=X$, and $X^{\prime}, \sigma_{2}=\sigma_{6}=0$, and solving these two simultaneous equations to obtain,

$$
\begin{aligned}
& F_{11}=\frac{1}{X X^{\prime}} \\
& F_{1}=\frac{1}{X}-\frac{1}{X}
\end{aligned}
$$

The transverse strength parameters are obtained by setting $\sigma_{2}=Y$, and $Y^{\prime}, \sigma_{1}=\sigma_{6}=0$, and solving these two simultaneous equations to obtain, 


$$
\begin{aligned}
& F_{22}=\frac{1}{Y Y^{\prime}} \\
& F_{2}=\frac{1}{Y}-\frac{1}{Y^{\prime}}
\end{aligned}
$$

The shear strength parameter is obtained by setting $\sigma_{1}=\sigma_{2}=0$, and $\sigma_{6}=\mathrm{S}$,

$$
\mathrm{F}_{66}=\frac{1}{\mathrm{~S}^{2}}
$$

The interaction term $F_{12}$ can only be obtained from combined-stress tests, which are difficult to perform. The interaction term is normalized by

$$
F_{12}=\frac{F_{12}^{*}}{\sqrt{X^{\prime} Y^{\prime}}}
$$

in which Tsai-Wu gives $-1 \leq \mathrm{F}_{12}^{*} \leq 1$ for enveloped in stress space is closed such that no combination of loading can result in infinite strength.

The Tsai-Wu quadratic failure criterion can be represented in strain space by using the stress-strain relationship

$$
\begin{aligned}
& F_{i j} \sigma_{i} \sigma_{j}=F_{i j}\left[Q_{i k} \varepsilon_{k}\right]\left[Q_{j 1} \varepsilon_{1}\right]=\left[F_{i j} Q_{i k} Q_{j 1}\right] \varepsilon_{k} \varepsilon_{1}=G_{k 1} \varepsilon_{k} \varepsilon_{1} \\
& F_{i} \sigma_{i}=F_{i}\left[Q_{i j} \varepsilon_{j}\right]=\left[F_{i} Q_{i j}\right] \varepsilon_{j}=G_{j} \varepsilon_{j}
\end{aligned}
$$

in which $Q_{i j}$ denote reduced stiffnesses relative to material axes 1 and 2 (Jones ${ }^{[63]}$ pp. 46). This form of the criterion is not based on plane strain, but is a representation of the plane stress criterion in strain space. For an orthotropic material, the strain space form for the criterion is written as,

$$
\mathrm{G}_{11} \varepsilon_{1}^{2}+2 \mathrm{G}_{12} \varepsilon_{1} \varepsilon_{2}+\mathrm{G}_{22} \varepsilon_{2}^{2}+\mathrm{G}_{66} \varepsilon_{6}^{2}+\mathrm{G}_{1} \varepsilon_{1}+\mathrm{G}_{2} \varepsilon_{2}=1
$$


where,

$$
\begin{aligned}
& \mathrm{G}_{11}=\mathrm{F}_{11} \mathrm{Q}_{11}^{2}+2 \mathrm{~F}_{12} \mathrm{Q}_{11} \mathrm{Q}_{12}+\mathrm{F}_{22} \mathrm{Q}_{12}^{2} \\
& \mathrm{G}_{22}=\mathrm{F}_{11} \mathrm{Q}_{12}^{2}+2 \mathrm{~F}_{12} \mathrm{Q}_{12} \mathrm{Q}_{22}+\mathrm{F}_{22} \mathrm{Q}_{22}^{2} \\
& \mathrm{G}_{12}=\mathrm{F}_{11} \mathrm{Q}_{11} \mathrm{Q}_{12}+\mathrm{F}_{12}\left(\mathrm{Q}_{11} \mathrm{Q}_{22}+\mathrm{Q}_{12}^{2}\right)+\mathrm{F}_{22} \mathrm{Q}_{12} \mathrm{Q}_{22} \\
& \mathrm{G}_{66}=\mathrm{F}_{66} \mathrm{Q}_{66}^{2} \\
& \mathrm{G}_{1}=\mathrm{F}_{1} \mathrm{Q}_{11}+\mathrm{F}_{2} \mathrm{Q}_{12} \\
& \mathrm{G}_{2}=\mathrm{F}_{1} \mathrm{Q}_{12}+\mathrm{F}_{2} \mathrm{Q}_{22}
\end{aligned}
$$

This form of the criterion is preferred since lamina strains are obtained directly from the analysis, and are easily transformed to the material coordinate system. Eqs. (6.11) can be calculated in advance for each lamina. Using the stress space form of the criterion requires an additional transformation from strain space to stress space for each lamina failure calculation.

The strength ratio ( $R$ ), is defined by Tsai $^{\left[{ }^{39}\right]}$ as the ratio between allowable load and applied load. This parameter is used as a linear scale factor for loads and/or displacements. When $\mathbf{R}<=1$, failure occurs. When $\mathbf{R}>1$, the structure has a positive Safety Factor $=\mathbf{R}$. That is, the failure load is equal to $\mathbf{R}$ times the applied load (proportional loading assumed). The strength ratio is derived for the quadratic criterion by letting $\varepsilon_{i}=\varepsilon_{i}^{\max }, i=1,2,6$, in Eq.(6.10), and then substituting $\varepsilon_{i}=R \varepsilon_{i}^{\max }$ to get.

$$
\left[G_{i j} \varepsilon_{i} \varepsilon_{j}\right] R^{2}+\left[G_{i} \varepsilon_{i}\right] R=1
$$

This results in a quadratic equation in $\mathrm{R}$, with the coefficients expanded as,

$$
\begin{aligned}
& \mathrm{G}_{\mathrm{ij}} \varepsilon_{\mathrm{i}} \varepsilon_{\mathrm{j}}=\mathrm{G}_{11} \varepsilon_{1}^{2}+2 \mathrm{G}_{12} \varepsilon_{1} \varepsilon_{2}+\mathrm{G}_{22} \varepsilon_{2}^{2}+\mathrm{G}_{66} \varepsilon_{6}^{2} \\
& \mathrm{G}_{\mathrm{i}} \varepsilon_{\mathrm{i}}=\mathrm{G}_{1} \varepsilon_{1}+\mathrm{G}_{2} \varepsilon_{2}
\end{aligned}
$$


This basic failure theory is extended to progressive failure by introducing a method for degradation of lamina strength and stiffness. The flow diagram for a lamina degradation in Tsai's progressive material failure analysis is shown in Figure 6.3

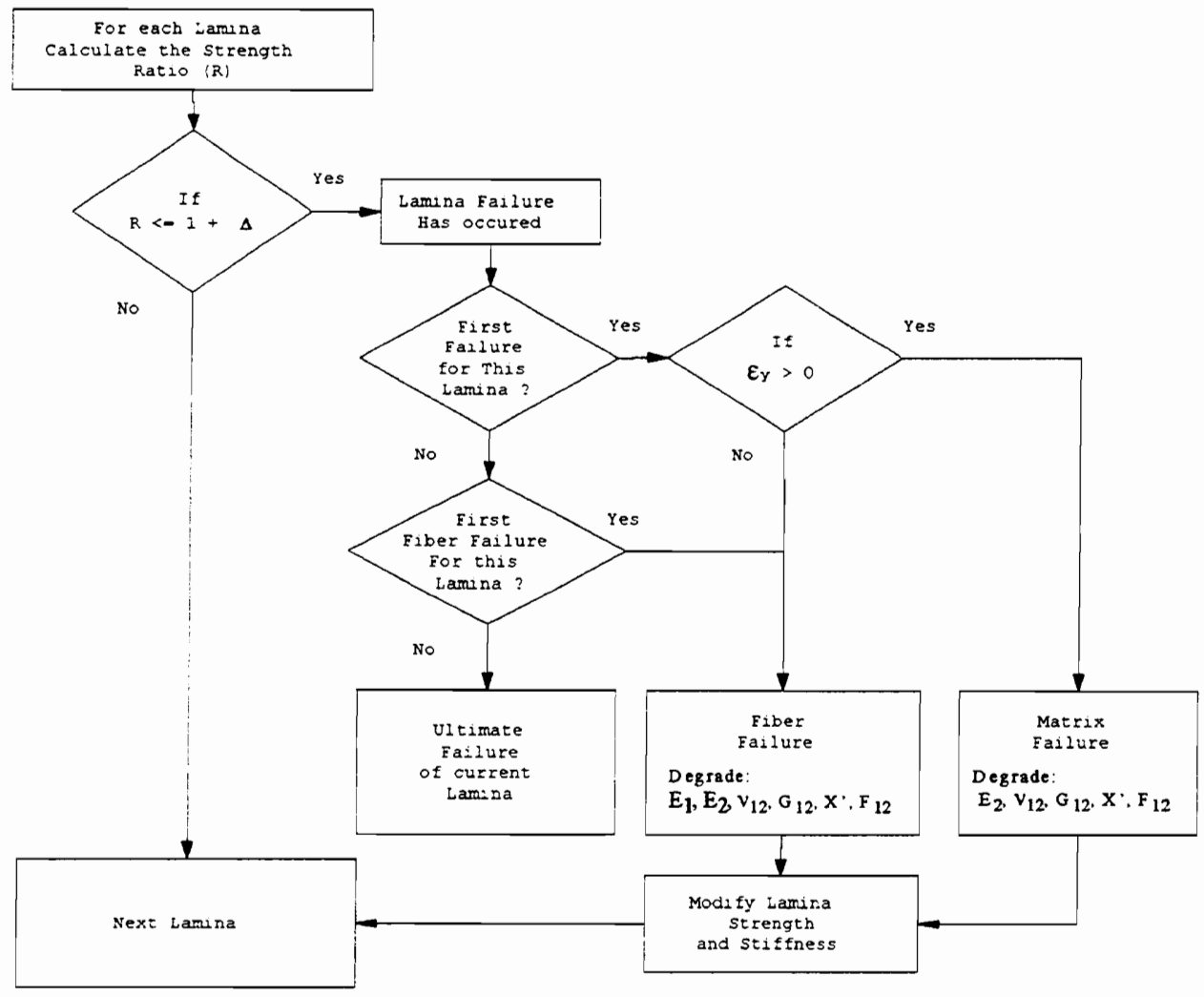

Figure 6.3 Flow chart for lamina degradation

If the strength ratio of a particular lamina is critical, failure is imminent for that lamina and the lamina properties are degraded. Based on the value of the transverse normal strain $\left(\varepsilon_{y}\right)$, and the failure history of the lamina in question, two types of material degradation are assumed:

1.) If $\varepsilon_{y}$ is positive, and there are no prior failures for this lamina; the event is considered to be a matrix failure, and a material degradation model which primarily reduces the transverse strength and stiffness is applied.

2.) If $\varepsilon_{y}$ is negative, or a prior failure has occurred in this lamina; the event is considered to be a fiber failure, and an alternate material degradation model which also reduces the axial stiffness is applied. 
A matrix failure can precede a fiber failure, but fiber failure is only allowed to occur once per lamina. The second indication of a fiber type failure in any lamina is interpreted as ultimate failure of the laminate containing that lamina.

Empirical constants are used in conjunction with lamina material properties to determine the lamina degradation factors used in Tsai's model. Two of these constants are the matrix degradation factor $E_{m}^{*}$, and the fiber degradation factor $E_{f}^{*}$. Another constant " $n$ " governs the reduction in axial compressive strength $X^{\prime}$. Also needed are the stress partitioning parameters $\eta_{2}$, and $\eta_{12}$., which account for different fiber and matrix stresses at the micromechanical level. These can also be treated as constants. The values that are recommended by Tsai ${ }^{[39]}$ as valid for a wide range of matrix/fiber combinations are given in Table 6.1.

Table 6.1 Empirical constants for lamina degradation factors

\begin{tabular}{ll}
\hline \hline \multicolumn{2}{c}{ Empirical Constants } \\
\hline $\mathrm{E}_{\mathrm{m}}^{*}$ & 0.15 \\
$\mathrm{E}_{\mathrm{f}}^{*}$ & 0.01 \\
$\eta_{2}$ & 0.5161 \\
$\eta_{12}$ & 0.3162 \\
$\mathrm{n}$ & 0.1 \\
\hline \hline
\end{tabular}

The modified rule of mixtures equations are used to determine the loss of transverse ply stiffness and in-plane shear stiffness due to material degradation. For matrix failure mode, the degraded properties are given by Eqs. (6.14), and (6.15).

$$
\begin{aligned}
& \frac{1}{\mathrm{E}_{2}^{\text {degraded-m }}}=\frac{1}{\left(1+v_{2}^{*}\right)}\left[\frac{1}{\mathrm{E}_{\mathrm{f} 2}}+\frac{v_{2}^{*}}{\mathrm{E}_{\mathrm{m}}^{*} \mathrm{E}_{\mathrm{m}}}\right] \\
& \frac{1}{\mathrm{G}_{12}^{\text {degraded-m }}}=\frac{1}{\left(1+v_{12}^{*}\right)}\left[\frac{1}{\mathrm{G}_{\mathrm{f} 12}}+\frac{v_{12}^{*}}{\mathrm{E}_{\mathrm{m}}^{*} \mathrm{G}_{\mathrm{m}}}\right]
\end{aligned}
$$


where, $E_{m}$ and $G_{m}$ are the matrix moduli, $E_{\mathrm{f} 2}$ is the transverse fiber modulus, and $G_{f 12}$ is the fiber shear modulus.

$$
v_{2}^{*}=\eta_{2} \frac{v_{m}}{v_{f}} \quad \text {, and } v_{12}^{*}=\eta_{12} \frac{v_{m}}{v_{f}}
$$

In Eqs.(6.16), $v_{\mathrm{m}}$, and $v_{\mathrm{f}}$ are the matrix and fiber volume fractions, respectively. For fiber failure mode, $\mathrm{E}_{\mathrm{m}}^{*}$ in Eqs. (6.14), and (6.15) is replaced by $\mathrm{E}_{\mathrm{f}}^{*}$ to obtain the degraded transverse lamina stiffness $E_{2}^{\text {degraded-f }}$, and the degraded lamina shear stiffness $G_{12}^{\text {degraded-f }}$.

The axial compressive strength is reduced by using the constant " $n$ ", to define a strength reduction factor as follows,

$$
X^{*}=\frac{X^{\text {degraded }}}{X^{\prime}}=\left(\frac{G_{12}^{\text {degraded }}}{G_{12}}\right)^{n}
$$

These lamina strength and stiffness reduction factors are summarized in Table 6.2.

\begin{tabular}{|c|c|c|}
\hline $\begin{array}{c}\text { Lamina } \\
\text { Material Data }\end{array}$ & $\begin{array}{c}\text { Matrix mode } \\
\text { Degradation factors }\end{array}$ & $\begin{array}{c}\text { Fiber mode } \\
\text { Degradation factors }\end{array}$ \\
\hline $\mathrm{E}_{1}$ & 1.0 & $\mathrm{E}_{\mathrm{f}}^{*}$ \\
\hline $\mathrm{E}_{2}$ & $E_{2}^{\text {degraded-m }} / E_{2}$ & $\mathrm{E}_{2}^{\text {degraded-f }} / \mathrm{E}_{2}$ \\
\hline$v_{12}$ & $\mathrm{E}_{\mathrm{m}}^{*}$ & $\mathrm{E}_{\mathrm{f}}^{*}$ \\
\hline $\mathrm{G}_{12}$ & $\mathrm{G}_{12}^{\text {degraded-m }} / \mathrm{G}_{12}$ & $\mathrm{G}_{12}^{\text {degraded-f }} / \mathrm{G}_{12}$ \\
\hline $\mathrm{X}$ & 1.0 & 1.0 \\
\hline $\mathrm{X}^{\prime}$ & $\mathrm{X}_{\mathrm{m}}^{*}$ & $\mathrm{X}_{\mathrm{f}}^{*}$ \\
\hline $\mathrm{Y}$ & 1.0 & 1.0 \\
\hline$Y^{\prime}$ & 1.0 & 1.0 \\
\hline $\mathbf{S}$ & 1.0 & 1.0 \\
\hline $\mathrm{F}_{12}^{*}$ & $\mathrm{E}_{\mathrm{m}}^{*}$ & $E_{f}^{*}$ \\
\hline
\end{tabular}

Table 6.2 Lamina degradation factors. 
The degradation is accomplished by multiplying the lamina material data in column 1 of Table 6.2, by the associated factors from either column 2 for matrix failure mode, or column 3 for fiber failure mode. As implied by Table 6.2, a material has three distinct states. Properties for each state can be calculated in advance, and used as they are needed.

One complication of using this material failure model in the current progressive failure analysis involves the requirement to calculate transverse strain $\varepsilon_{y}$. Notice in Figure 6.3 that $\varepsilon_{\mathrm{y}}$ is used in determining the lamina failure mode. For the current beam theory, $\varepsilon_{\mathrm{y}}$ is assumed to be zero. Attempts to calculate $\varepsilon_{\mathrm{y}}$ using shell equilibrium equations were not successful due to boundary condition problems associated with the finite element modeling. Calculations must be done on an element by element basis, but the boundary conditions for $\mathrm{N}_{\mathrm{y}}$ are not in general available at the element boundaries. Because the equilibrium method was not successful, an altemative method was implemented. This method relies on Eq (2.99) which expresses the reactive strains $\left(\varepsilon_{y}, \kappa_{y}, \gamma_{x y}\right)$ in terms of the active strains $\left(\varepsilon_{\mathrm{x}}, \mathrm{K}_{\mathrm{x}}, \mathrm{K}_{\mathrm{xy}}\right)$. The advantage of this method is that approximations for $\gamma_{\mathrm{xy}}$ and $\kappa_{y}$, are obtained as well. Reactive strains calculated in this way were compared to equivalent strains calculated using plate theory and were found to be acceptable. Some of these comparisons are shown in Table 6.3, Table 6.4, and Table 6.5

Table 6.3 Quasi isotropic coupon (NASA CR 4287) [49], 8 plies [45/-45/0/90]

\begin{tabular}{|c|c|c|}
\hline & $\varepsilon_{\mathrm{x}}(\%)$ & $\varepsilon_{\mathrm{y}}(\%)$ \\
\hline CLT (GENLAM) ${ }^{[64]}$ & 1.0000 & -0.3027 \\
\hline BEAM (Reactive $\varepsilon_{y}$ ) & 1.0000 & -0.3027 \\
\hline
\end{tabular}

Table 6.4 van Wamelen' ${ }^{165]}$ on axis coupon, 16 plies [0/90/45/0/90/-45/90/0]

\begin{tabular}{llc}
\hline \hline & \multicolumn{1}{c}{$\varepsilon_{\mathrm{x}}(\%)$} & $\varepsilon_{\mathrm{y}}(\%)$ \\
\hline CLT (GENLAM) ${ }^{[64]}$ & 1.0000 & -0.1456 \\
BEAM (Reactive $\left.\varepsilon_{\mathrm{y}}\right)$ & 1.0000 & -0.1456 \\
\hline \hline
\end{tabular}


Table 6.5 van Wamelen 's ${ }^{65]}$ off axis coupon, 16 plies $[10 / 100 / 55 / 10 / 100 /-35 / 100$ $10]_{\mathrm{s}}$

\begin{tabular}{llll}
\hline & \multicolumn{1}{c}{$\varepsilon_{\mathrm{x}}(\%)$} & \multicolumn{1}{c}{$\varepsilon_{\mathrm{y}}(\%)$} & \multicolumn{1}{c}{$\gamma_{\mathrm{xy}}(\%)$} \\
\hline CLT (GENLAM) ${ }^{[64]}$ & 1.0000 & -0.2019 & -0.3626 \\
BEAM (Reactive $\left.\varepsilon_{\mathrm{y},} \gamma_{\mathrm{xy}}\right)$ & 1.0000 & -0.2019 & -0.3626 \\
\hline \hline
\end{tabular}

Using Eq (2.99) to approximate $\varepsilon_{\mathrm{y}}$ in this fashion requires an assumption that the reactive resultants $\left(N_{y}, M_{y}, N_{x y}\right)$ are zero. This is a good approximation for the simple examples presented, but can easily break down for more complex boundary conditions. Note that it is only the sign of $\varepsilon_{\mathrm{y}}$ that is of interest in selecting the correct material failure mode.

\subsection{EVALUATION AND COMPARISON TO TEST DATA}

An evaluation of this method for progressive failure analysis was conducted by comparing analytical results to the available data from a limited number of test cases. To check the basic operation of the progressive failure theory, comparisons were made to results obtained for tensile coupon test specimens. Material properties for these coupons are listed in Table 6.10, and the laminate stacking sequence is given in Table 6.7. The interaction term $\mathrm{F}_{12}^{*}$ is set to -0.5 as $\mathrm{Tsai}^{\left[{ }^{39}\right]}$ recommends based on arguments on the admissible range of the term.

Table 6.6 Material properties - BASF G30500/5208 graphite epoxy

\begin{tabular}{ccc|ccccc|c}
\hline \hline \multicolumn{6}{c|}{ Stiffness (Msi) } & \multicolumn{5}{c|}{ Strength (ksi) } & \\
\hline $\mathrm{E}_{1}$ & $\mathrm{E}_{2}$ & $\mathrm{G}_{12}$ & $\mathrm{X}$ & $\mathrm{X}^{\prime}$ & $\mathrm{Y}$ & $\mathrm{Y}^{\prime}$ & $\mathrm{S}$ & $v_{12}$ \\
\hline 22.04 & 1.322 & 1.04 & 295.0 & 295.0 & 7.50 & 35.67 & 11.3 & 0.286 \\
\hline \hline
\end{tabular}

Table 6.7 Tensile coupon laminate stacking sequences

\begin{tabular}{lc}
\hline \hline \multicolumn{1}{c}{ Coupon } & Stacking Sequence \\
\hline On axis & {$[0 / 90 / 45 / 0 / 90 /-45 / 90 / 0] \mathrm{s}$} \\
$10^{\circ}$ off axis & {$[10 / 100 / 55 / 10 / 100 /-35 / 100 / 10] \mathrm{s}$} \\
\hline \hline
\end{tabular}


These coupons were analyzed by van Wamelen ${ }^{[65]}$ using Tsai's progressive method. The coupon specimens were modeled as thin beams in tension using the current program, and the failure predictions were compared to van Wamelens results. The failure predictions from the beam were in close agreement with those obtained by van Wamelen.

Table 6.8 Failed ply and loads for progressive failure comparison to prediction by van Wamelen ${ }^{[65]}$ for on axis laminate tensile coupon

\begin{tabular}{lccc}
\hline \hline \multicolumn{4}{c}{ Ply Failure Stress (psi) } \\
Failed Ply & Beam Model & van Wamelen & \% Difference \\
\hline 90.00 & 59582.12 & 59675.00 & 0.16 \\
45.00 & 63541.30 & 63594.00 & 0.08 \\
0.00 & 120060.94 & 120682.00 & 0.51 \\
\hline \hline
\end{tabular}

Table 6.9 Failed ply and loads for progressive failure comparison to prediction by van Wamelen ${ }^{[65]}$ for $10^{\circ}$ off axis laminate tensile coupon

\begin{tabular}{cccc}
\hline \hline \multicolumn{4}{c}{ Ply Failure Stress (psi) } \\
Failed Ply & Beam Model & van Wamelen & \% Difference \\
\hline 100.00 & 53883.18 & 53900.00 & 0.03 \\
55.00 & 50164.00 & 50760.00 & 1.17 \\
-35.00 & 74207.35 & 74270.00 & 0.08 \\
\hline \hline
\end{tabular}

The progressive failure analysis was next applied to an "I" cross-section curved composite fuselage-type frame originally tested by E.Moas et. $a \|^{20}$ ]. Material for this frame is AS4/ 5208, graphite epoxy with material properties as listed in Table 6.10. Strength data was obtained from coupon tests of specimens cut from the web of a similar frame. The specimen frame was made from the same material and was used for the same series of tests. The coupons were tested by Collins et. al. and the results are presented graphically in Ref.[ 49 ]. The strength data used for this analysis is also listed in Table 6.10. The interaction 
term $\mathrm{F}_{12}^{*}$ was taken as -0.5

Table 6.10 Material properties - AS4/5208 graphite epoxy

\begin{tabular}{rcc|cccccc|c}
\hline \hline \multicolumn{6}{c|}{ Stiffness (Msi) } & \multicolumn{5}{c|}{ Strength (ksi) } & \\
\hline $\mathrm{E}_{1}$ & $\mathrm{E}_{2}$ & $\mathrm{G}_{12}$ & $\mathrm{X}$ & $\mathrm{X}$ & $\mathrm{Y}$ & $\mathrm{Y}^{\prime}$ & $\mathrm{S}$ & $v_{12}$ \\
\hline 18.4 & 1.64 & 0.87 & 197.9 & 160.0 & 5.50 & 29.9 & 14.0 & 0.300 \\
\hline
\end{tabular}

Table 6.11 Test frame laminate stacking sequences

\begin{tabular}{cc}
\hline \hline & Stacking Sequence \\
\hline Cap & {$[+45 /-45 / 0 / 90] \mathrm{s}$} \\
Web & {$[+45 /-45 / 0 / 90]_{\mathrm{s}}$} \\
Attachment & {$[+45 /-45 / 0 / 90 / 90 / 0 /+45 /-45]_{\mathrm{T}}$} \\
Skin & {$[+45 /-45 / 0 / 90 /+45 /-45 / 0 / 90] \mathrm{s}$} \\
\hline \hline
\end{tabular}

The basic geometry of this frame with some useful section terminology is shown in Figure $6.4 \mathrm{t}$.
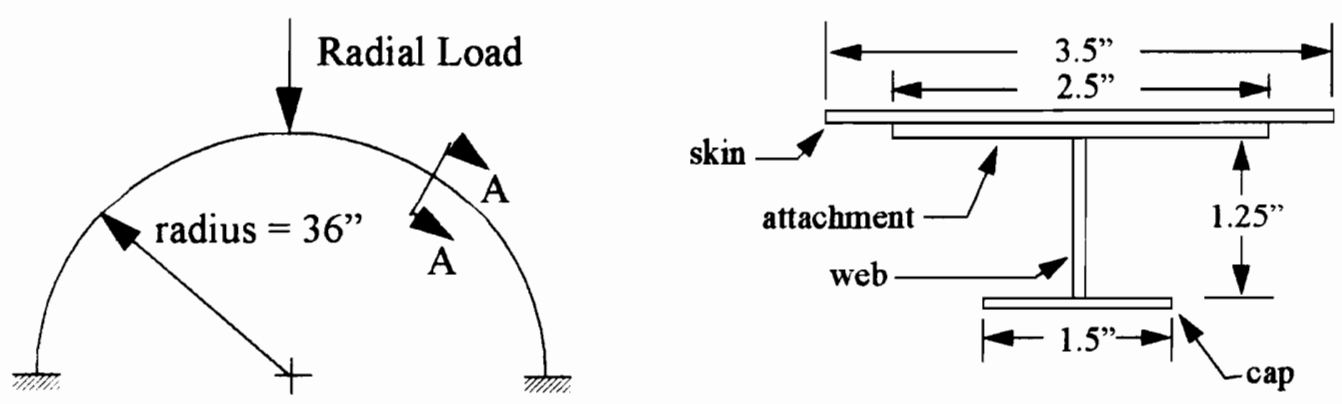

Section AA

Figure 6.4 Curved test frame geometry and cross section

The section branch thickness measurements for the frame designated " $1 b$ " were obtained from the authors of Ref. [ 20 ], and the nominal lamina thicknesses were modified to 
match the average measured dimensions. Lamina thicknesses are presented in Table 6.12 Table 6.12 Branch lamina thicknesses for curved test frame.

\begin{tabular}{lccc}
\hline \hline Branch & $\begin{array}{c}\text { Average measured } \\
\text { branch thickness } \\
\text { (in.) }\end{array}$ & $\begin{array}{c}\text { Number of } \\
\text { Plies }\end{array}$ & $\begin{array}{c}\text { Lamina } \\
\text { thickness for } \\
\text { analysis (in) }\end{array}$ \\
\hline Cap & 0.0420 & 8 & 0.005250 \\
Web & 0.0400 & 8 & 0.005000 \\
Skin & 0.0825 & 16 & 0.005156 \\
Attachment + skin & 0.1224 & 24 & 0.005104 \\
\hline
\end{tabular}

The cross section was modeled using the natural junctions and thickness changes as section element boundaries, and 20 curved beam elements were used along the arc length. Clamped end conditions were assumed at each end of the frame. The load response test data for the this frame is shown with the corresponding progressive analysis results from the current program in Figure 6.5, Figure 6.6, and Figure 6.7.

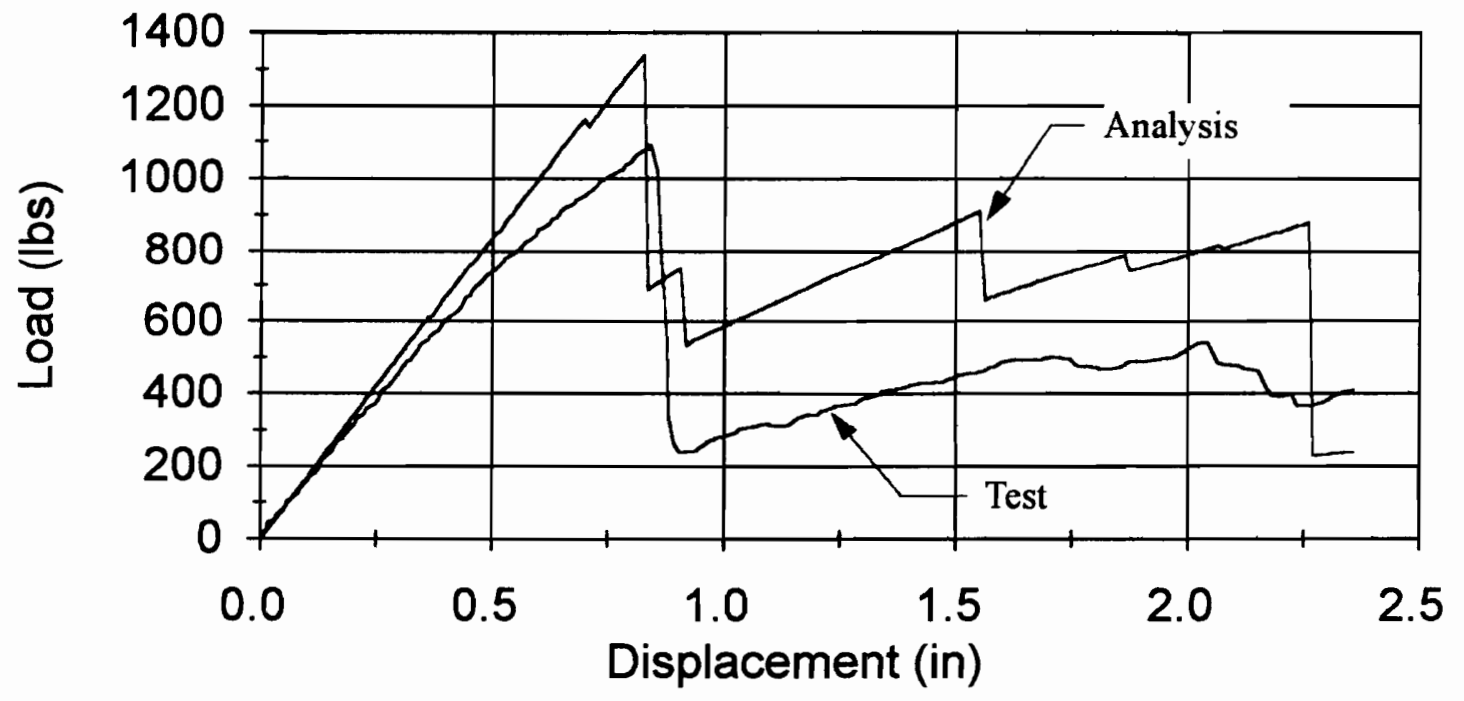

Figure 6.5 Comparison of progressive failure to experimental results for frame $1 b$ 


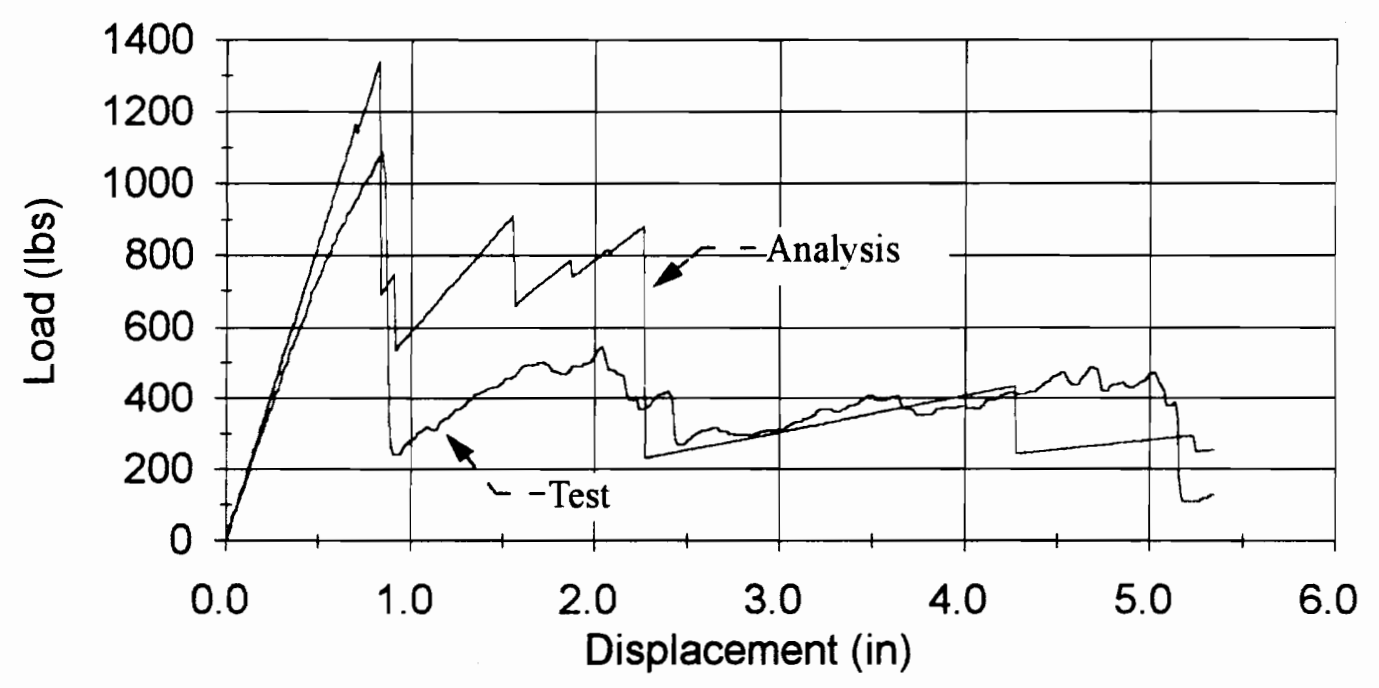

Figure 6.6 Extended comparison of progressive failure to experimental results for frame $1 b$

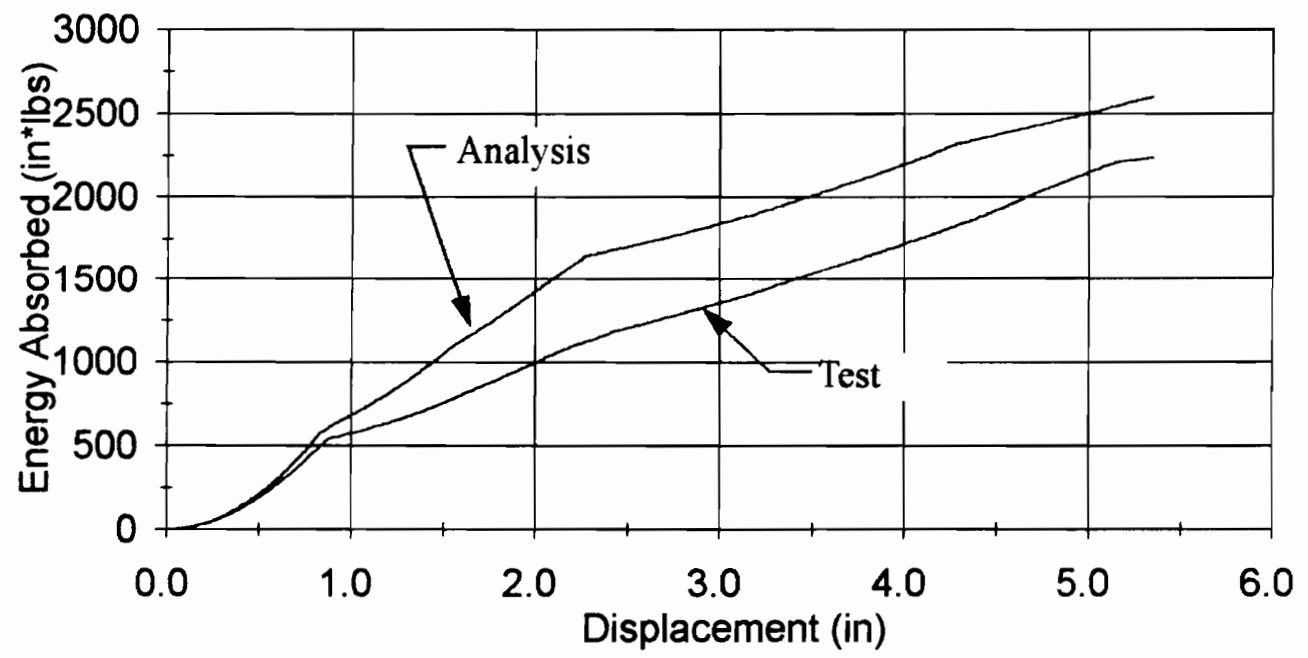

Figure 6. 7 Comparison of energy absorption.

The results shown in Figure 6.5, Figure 6.6 and Figure 6.7 indicate a relatively good 
agreement between the progressive analysis and the experimental data, especially up to the first major failure event. The failure displacement at this point is essentially correct, but the predicted failure load is high by approximately $200 \mathrm{lbs}$. This discrepancy may be due to the slight non-linearity of the structural response as reflected in the experimental data. Notice that for the sequence after the first major failure, the average discrepancy remains approximately $200 \mathrm{lbs}$. The energy comparison curves diverge at the first major failure, and then become approximately parallel again at a displacement of roughly 2.25 ". These results are considered good given the simplifying assumptions made for the current theory.

\subsection{SENSITIVITY TO MESH DISCRETIZATION}

One aspect of the progressive failure method developed in this chapter that requires study, is the possible dependence of the progressive failure solution on the number of beam elements used. This mesh dependency is a consequence of the fact that branches are assumed to fail along the entire length of a beam element. Recall that failure is calculated at six points along the length of a beam element, with failure at any one point sufficient to fail that section element for the entire span of the beam element. When this beam element is replaced by two elements, the global failure pattern can be altered since the branch is now free to fail on one beam only. This effect is illustrated in Figure 6.8 .
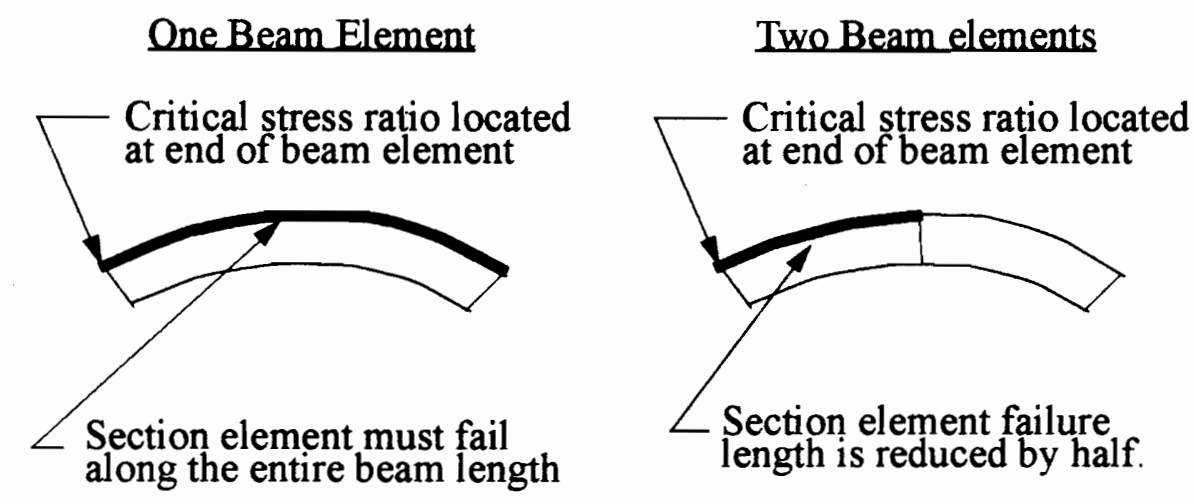

Figure 6.8 Effect of mesh discretization 
Figure 6.9, Figure 6.10, and Figure 6.11, are load response diagrams from progressive failure analysis which use the same beam geometry, cross section, and material stacking sequence, but have increasing numbers of beam elements. $(16,30$, and 50 elements along the circumference). As the beam elements are increased, the shape of the load response changes. The points located on the lines in Figure 6.9, Figure 6.10, and Figure 6.11, represent discrete failure events as calculated by the analysis program. However, as seen in Table 6.13 the change in energy absorbed at the cut off displacement of three inches is minimal.

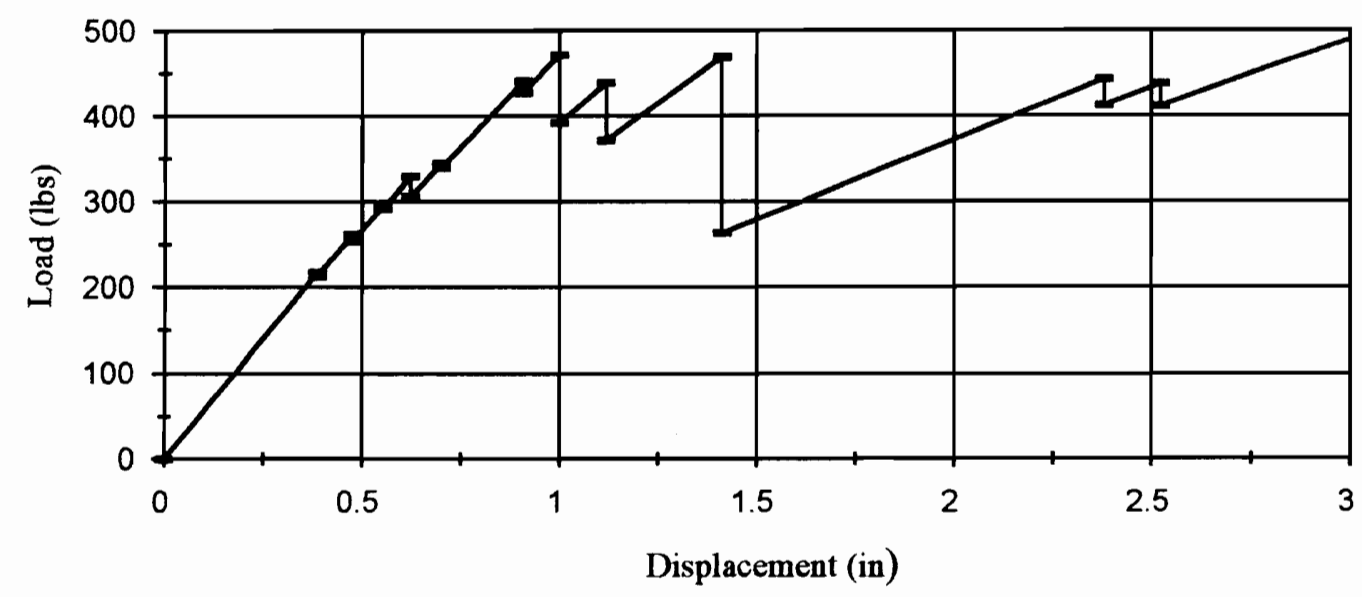

Figure 6.9 Progressive failure using 16 beam elements

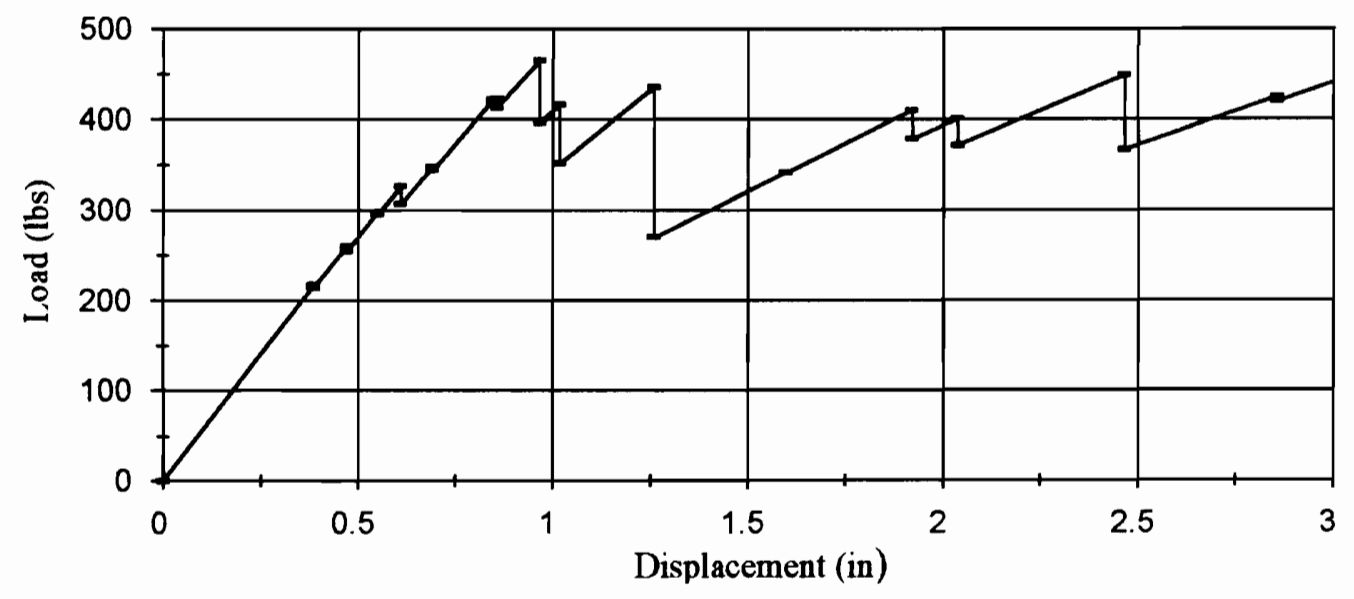

Figure 6.10 Progressive failure using 30 beam elements 


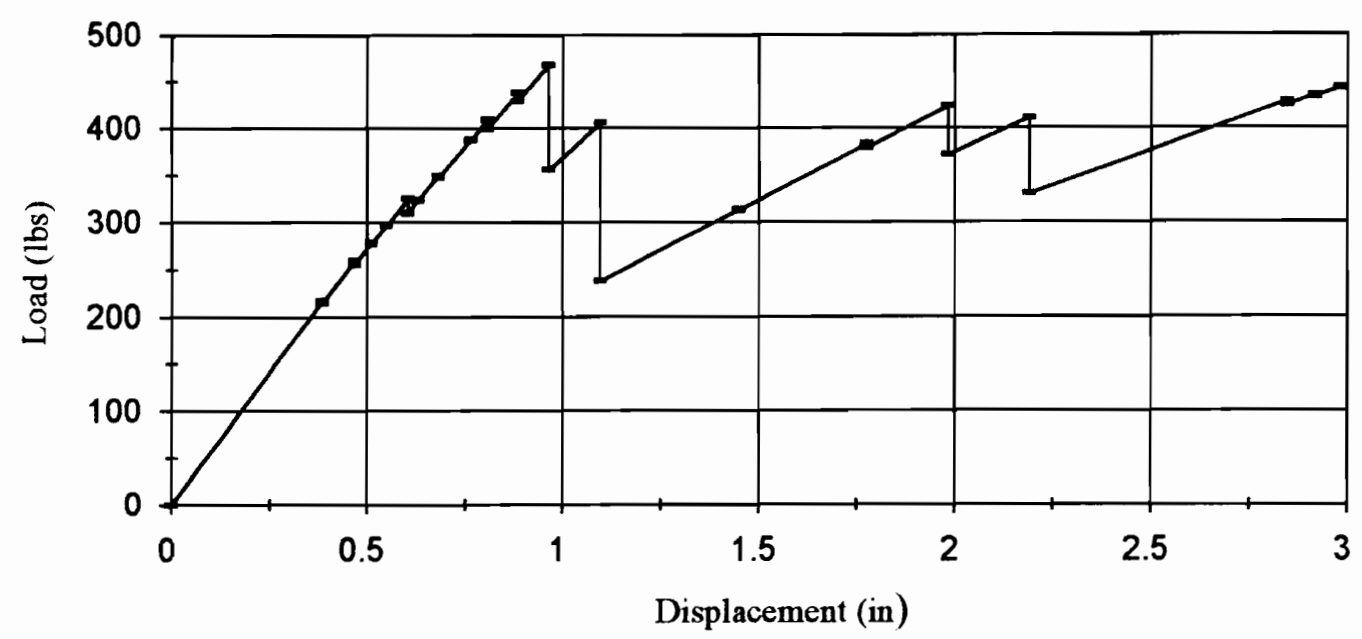

Figure 6.11 Progressive failure using 50 beam elements

Table 6.13 Energy absorbed as a function of mesh discretization

\begin{tabular}{ccc}
\hline \hline \multicolumn{3}{c}{ Energy Absorbed at 3.0" (in*lbs) } \\
16 elements & 30 elements & 50 elements \\
\hline 1044.4 & 1039.74 & 1020.71 \\
\hline \hline
\end{tabular}

\subsection{SENSITIVITY OF ENERGY ABSORBED TO FAILURE ENVELOPE}

The energy absorption calculated using the current progressive failure methodology shows a slight sensitivity to the size of the failure envelope used. Recall that the failure envelope specifies a range of strength ratios for which laminates are considered failed and are modified. Initial increases in the failure envelope size will reduce the total number of analysis/ modification cycles required for each progressive failure analysis, without affecting the calculation of energy absorbed. The benefits of increasing the failure envelope decrease sharply after the $2.5 \%$ level is reached, and disappear totally after the $5 \%$ envelope is reached. If the failure envelope becomes too large it can also affect the accuracy of calcu- 
lated energy absorbed. Table 6.14 shows the influence of the changing failure envelope on accuracy of energy absorption and analysis cost As stated earlier, a failure zone of $2.5 \%$ was used for this work.

Table 6.14 Influence of failure envelope on calculated energy absorption

\begin{tabular}{ccc}
\hline $\begin{array}{c}\text { Size of failure envelope } \\
(\%)\end{array}$ & $\begin{array}{c}\text { Energy Absorbed } \\
\text { (in*lbs) }\end{array}$ & $\begin{array}{c}\text { Number of analysis } \\
\text { cycles required }\end{array}$ \\
\hline 0.5 & 426.19 & 39 \\
1.0 & 426.19 & 31 \\
1.5 & 426.19 & 24 \\
2.0 & 426.19 & 19 \\
2.5 & 426.19 & 16 \\
3.0 & 426.19 & 15 \\
3.5 & 426.19 & 13 \\
4.0 & 426.19 & 13 \\
4.5 & 426.19 & 12 \\
5.0 & 426.19 & 11 \\
5.5 & 426.19 & 11 \\
6.0 & 426.19 & 11 \\
7.0 & 426.19 & 10 \\
8.0 & 426.19 & 10 \\
9.0 & 419.12 & 11 \\
10.0 & 419.17 & 10 \\
\hline \hline
\end{tabular}




\section{CHAPTER 7}

\section{OPTIMAL FRAME DESIGN FOR CRASHWORTHINESS}

\subsection{PROBLEM FORMULATION}

General concepts of crashworthiness were introduced and discussed in Chapter 1. In this chapter, the specific problem of optimizing composite fuselage frames for crashworthiness is addressed. Optimal fuselage frame crashworthiness is obtained by; 1.) Maximizing the capacity of the frame to absorb vertical crush energy, and 2.) Limiting the maximum failure load.

For optimization of crashworthiness, a single radially directed load (F) is applied at the frame center to simulate crash loading caused by impact with the ground. Absorbed energy $\left(E_{a}\right)$ is calculated as the area under the load-displacement response prior to ultimate failure. The displacement $\delta$ used for calculation of $E_{a}$, is measured at the point of load application, in the direction of the applied load. In a progressive failure analysis of composite frames for energy absorption, there are many possible definitions of ultimate failure. One possibility is a large and sudden drop in radial frame stiffness due to localized failures. For the present work, ultimate failure is defined as the first load drop of $25 \%$ or larger in the failure sequence. This definition is expected to promote optimal failure sequences which approximate the ideal elastic plastic load-response. The displacement at 
which this failure occurs will be designated $\delta_{\text {ult }}$. The design problem is illustrated in Figure 7.1

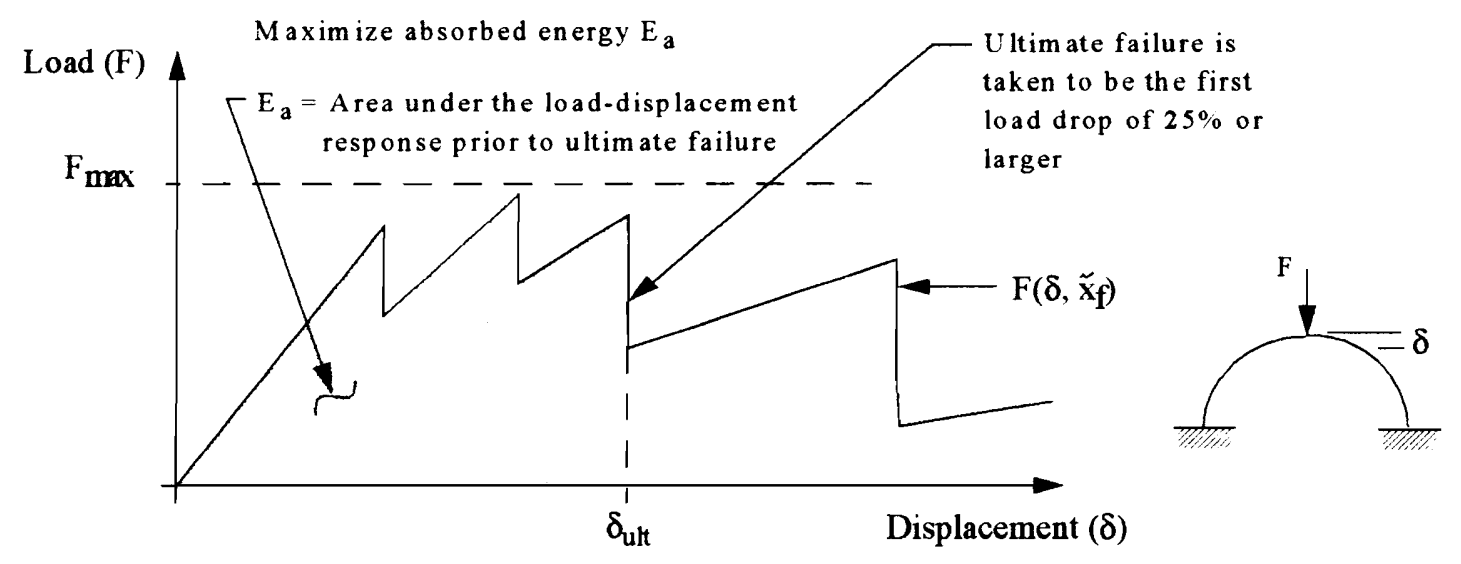

Figure 7.1 Optimal design problem for maximizing frame crashworthiness

From the design requirements and objectives, we can formulate an optimal design problem for maximizing crashworthiness of composite fuselage frames:

$$
\begin{array}{ll}
\text { Maximize: } & \mathrm{E}_{\mathrm{a}}=\int \mathrm{F}\left(\delta, \tilde{\mathrm{x}}_{\mathrm{f}}\right) \mathrm{d} \delta \\
\text { Subject to: } & \mathrm{F}\left(\delta, \tilde{\mathrm{x}}_{\mathrm{f}}\right) \leq \mathrm{F}_{\max }, \\
& 0 \leq \delta \leq \delta_{\mathrm{ult}},
\end{array}
$$

where, $E_{a}$ is the absorbed energy, $F\left(\delta, \tilde{x}_{f}\right)$ is the applied load which is a function of the frame displacement at the load point $\delta$, and the vector of frame design variables $\tilde{\mathbf{x}}_{\mathrm{f}}$, and $F_{\max }$ is an upper bound on the allowable magnitude of the failure load. This formulation will maximize absorbed energy, which is given by the area under the load-response curve up to ultimate failure, and limit peak accelerations which are directly related to peak failure loads (see Chapter 1).

The principal composite frame design variables for energy optimization are laminate 
stacking sequence, and cross section shape. For the present work, frame curvature as a function of circumference will remain constant, and material properties will be specified. It is possible to have an independent laminate for each cross-section branch, and an independent cross section shape for each beam element. Practical design considerations limit ply orientations for each laminate to a discrete predetermined set of $n_{a}$ angles. Thus for each independent laminate stacking sequence, with " $n$ " plies, there are " $n$ " design variables with " $n_{\mathrm{a}}$ " discrete possible values. Each independent cross section shape is also characterized by one or more independent shape design variables. This implies that the number of frame design variables $\tilde{x}_{f}$, can easily become large. A basic composite frame design problem with one 8 - ply symmetric laminate, and three cross section shape variables, will have 7 design variables.

The objective function $E_{a}$ in Eq. ( 7.1 ) is in general a nonlinear function of the design variables $\tilde{\mathbf{x}}_{\mathrm{f}}$. This nonlinearity is typical in optimization problems involving laminate stacking sequence. Figure 7.2 contains a plot of total energy absorbed as a function of $\theta$, for an angle ply laminate $[+\theta /-\theta /+\theta /-\theta]_{s}$, in tension. These values are obtained by limiting maximum load drop at a failure event to $25 \%$ or less. Extending the failure analysis beyond this point resulted in even more extreme gradients. This example illustrates the nonlinear effect for a balanced symmetric laminate, with only one design variable. When additional ply variables are independent, the design space becomes more complex. Figure 7.3 represents a similar laminate, however in this case, three of the plies are held constant at a $45^{\circ}$ angle, while the outermost ply angle $\theta$ is varied from 0 to 90 degrees. Notice the very steep gradient as $\theta$ goes from 4 , to 5 degrees. This is a discontinuous change caused by the fail- 
ure constraint becoming active at a different location on the load response curve.

Energy Absorbed .vs. Ply Angle

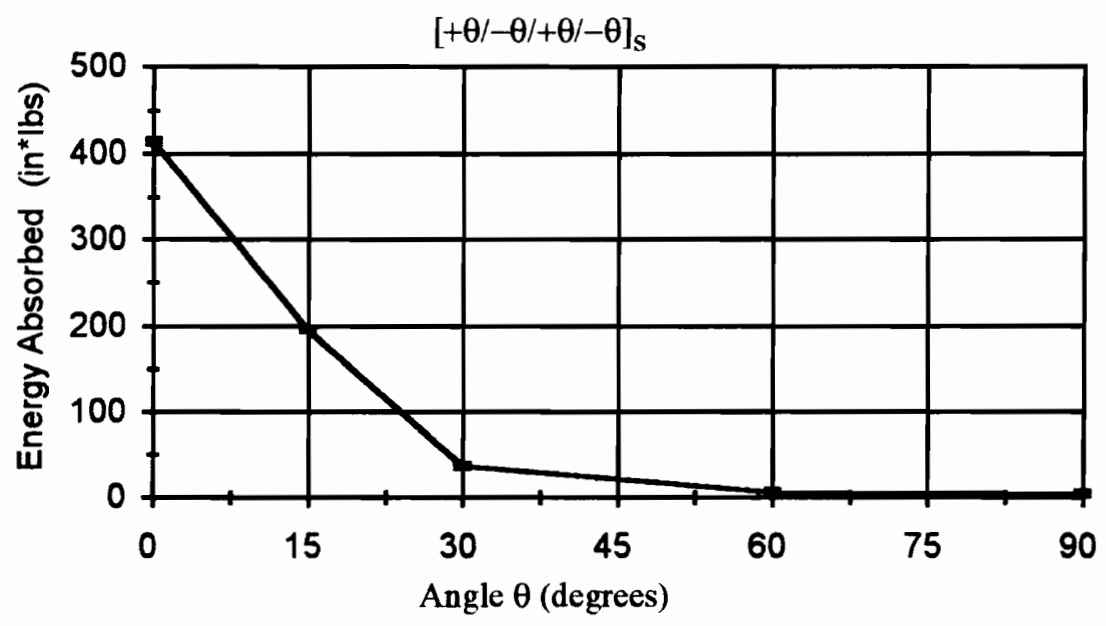

Figure 7.2 Nonlinearity of Energy absorption .vs. ply angle (angle ply laminate)

Energy Absorbed .vs. Ply Angle

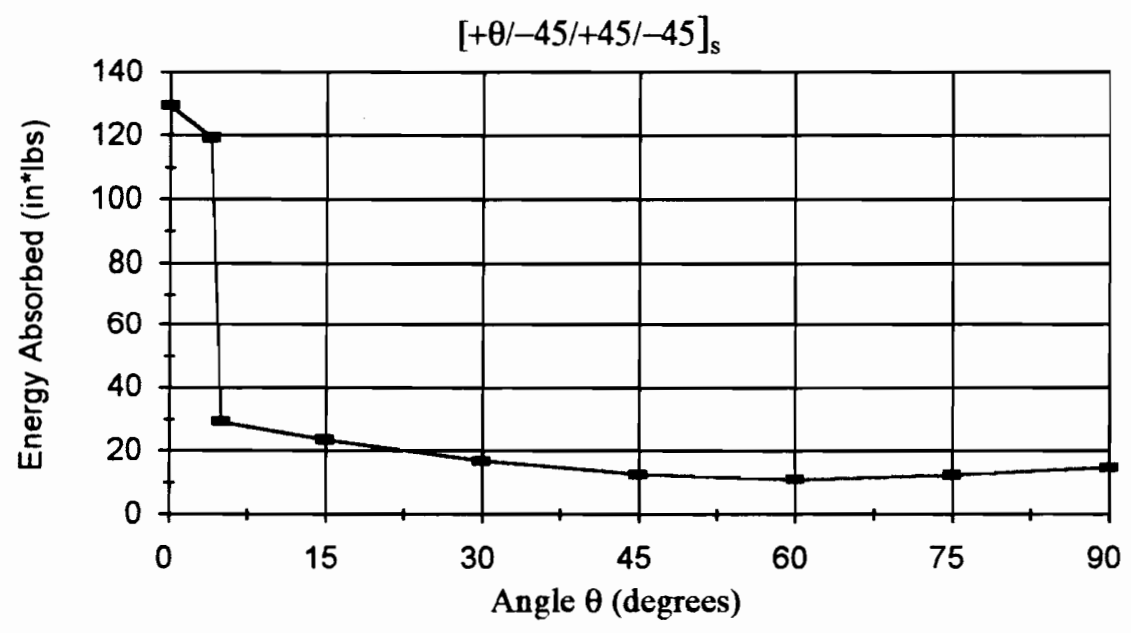

Figure 7.3 Discontinuity of Energy Absorption.

Nonlinearity for the shape design parameters appears less severe, and is demonstrated in

Figure 7.4 using an I cross section example. This is a straight beam loaded transversely at 
mid span. The height of the cross section is varied.

Energy Absorbed .vs. Section Height

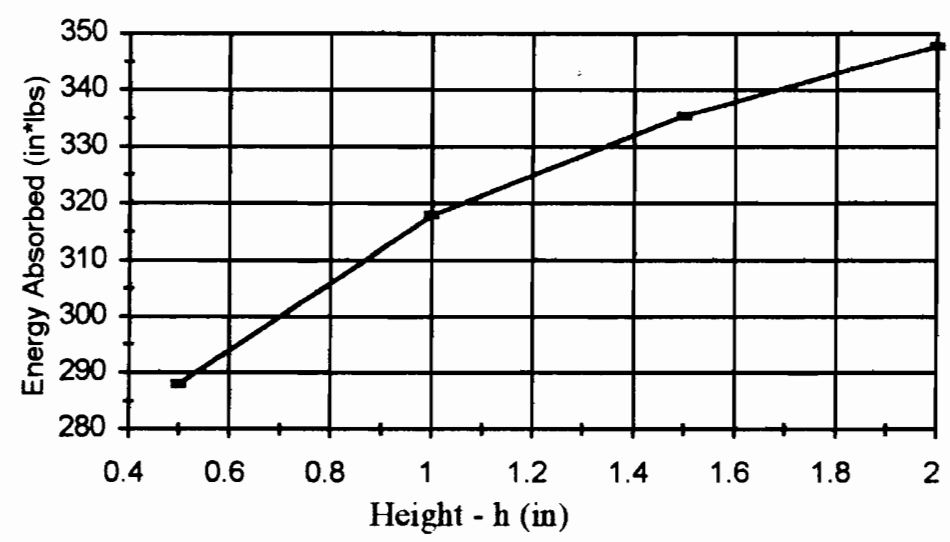

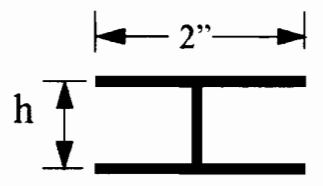

Example Section: $\mathrm{t}=.042 "$, Typical

Figure 7.4 Nonlinearity of Energy Absorbed.vs. Section Shape

These examples illustrate two important features of the current optimization problem.

First, the objective function is nonlinear in both design variable types. Second, there are discontinuities in the objective function which are caused by the failure criteria. The choice to use discrete ply angles will create additional discontinuities, and cause the objective to be discrete valued.

It may be feasible to solve this problem using traditional gradient based optimization methods, but there are disadvantages to this approach. First, the nonlinearity seen in Figure 7.2, and Figure 7.3 often results in local optima in the design space. Note the local optimum at $\theta=90^{\circ}$ in Figure 7.3. There are often many optimal or near optimal laminates for a composite design problem.(see Shin et. al. ${ }^{[66]}$ ) Gradient based methods tend to find the nearest local optimum, which may not be the global optimum. Second, there are discontinuities in the objective function, and this often leads to convergence problems with a gradient based method. Third, using the gradient based approach directly requires that ply angles be continuous variables. Strategies based on rounding are often used but there is no 
guarantee that the discrete optimum will be in the neighborhood of the of the continuous optimum $^{[67]}$.

Probabilistic optimization methods overcome many of the problems just delineated. Two probabilistic (or stochastic) search methods are genetic algorithms, and simulated annealing. They are alternatives to traditional optimization methods and have emerged as important tools for certain types of difficult optimization problems. Probabilistic methods do not require derivative information, and easily handle a discrete and discontinuous nonlinear design space. They frequently locate many optimal and near optimal designs simultaneously, giving the designer a choice. Considering these factors, the genetic optimization algorithm is considered a reasonable approach to the optimization problem of Eq. ( 7.1)

\subsection{GENETIC ALGORITHMS}

Genetic algorithms use techniques which simulate biological natural selection, and rely on the application of Darwin's principle of survival of the fittest. When a population of biological creatures is allowed to evolve over generations, individual characteristics that are useful for survival tend to be passed on to future generations. This occurs because the individuals carrying these traits are more likely to survive and breed. In biological populations, these characteristics are stored as chromosomal strings. The basic operations that result in a structured yet randomized exchange of genetic information between the chromosomal strings of reproducing parents include crossover, and occasional mutation.

Genetic algorithms were developed by Holland ${ }^{[68]}$, to mimic the mechanics of natural genetics with mathematical operations as counterparts to the natural ones. These operations involve a similar randomized exchange of numbers in strings that represent the design variables. These operations may appear like a completely random search of extremum in parameter space based on function values only. However, genetic algorithms are 
experimentally proven to be robust, and the reader is referred to Goldberg $\left.{ }^{[69}\right]$ for a complete discussion of their theoretical properties. Here we discuss the genetic representation of the stacking sequence and cross-section shape, for the maximization of energy absorption.

Unlike many search algorithms that move from one point to another in the design space, genetic algorithms work with a population of designs represented as numeric strings.

Keeping many solution points, rather than singling out a point early in the process, reduces the risk of getting stuck at a local optimum. This aspect of the genetic algorithms is responsible for the increased chance for obtaining global or near global optima. Genetic algorithms do not require derivative information, and easily handle a discontinuous or discrete valued non-linear design space. The outcome of a genetic search is a population of good designs rather than a single design. This aspect can be very useful to the designer. These features make genetic algorithms attractive for optimization problems involving laminate stacking sequence. Examples of the application of genetic algorithms to struc-

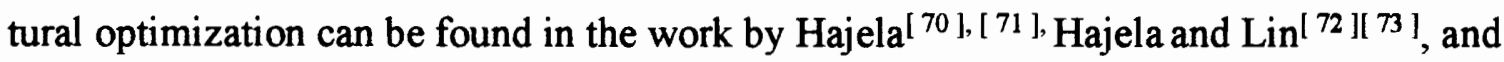
Le Richel ${ }^{74],[75]}$.

The drawback to genetic algorithms is that a population of designs evolving over generations towards an optimum requires a relatively high number of analyses. A typical optimization might require thousands of objective function evaluations. This places a practical limit of the type of problems which are feasible to solve with this method. A fast analysis method is essential, and therefore the use of efficient analysis models is important. The Vlasov type composite curved beam analysis is very efficient, and permits the use of genetic algorithms for the energy absorption problem at hand

\subsection{FUNDAMENTALS OF GENETIC ALGORITHMS}

Genetic optimization literature frequently uses terminology that is borrowed from the ori- 
gins of this method in the field natural genetics. The optimization process operates on a group of designs referred to as a population. Each design within the population is referred to as an individual. Individuals are described using coded information in a genetic string or chromosome. This coding is a parallel of the DNA in natural genetics. Each piece of coded information in the genetic string is referred to as a gene or feature, and its locus specifies position within the string. Genes are often restricted to discrete allowable values (alleles or feature values), which may vary with locus. The range of alleles for a particular problem is an alphabet. Individuals are evaluated, and assigned a fitness, which the measure of the relative worth of an individual.

The population of designs evolves toward the optimum from one generation to the next. Each generation is a search of multiple points in the design space. Probabilistic transition rules guide the evolution. Each time the algorithm is used it will follow a different path. This does not imply that the search is random. Probabilistic transitions guide the search to regions of the domain where improvement is likely.

Genetic algorithms use a coding of the parameter set, and not the parameters themselves. The problem is coded as a finite length string over one or more finite alphabets. An example of this is the representation of integers in binary form. Using two alleles ( 0 , and 1 ), and a string of appropriate length, a range of integers can be represented (it is also possible to approximate continuous domains using binary arithmetic, or scaling ). Coding also isolates problem specific features from the genetic algorithm. Thus a "generic" genetic algorithm can be used on virtually any problem with little modification. An example coding of 
laminate stacking sequence is presented in Figure 7.5

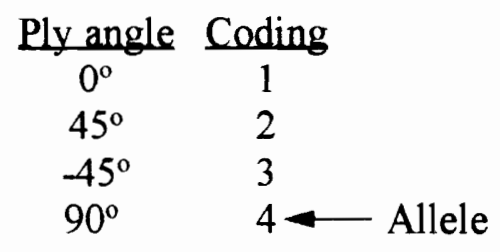

Size of design space $=4^{8}$, or 65,536
Stacking Sequence:

$$
[+45 /-45 / 0 / 90 / 90 / 0 /-45 / 45]_{\mathrm{T}}
$$

Coded Genetic String:

Figure 7.5 Example coding sequence

Transition from one generation to the next is frequently accomplished using three basic operators.

Selection; The selection operator selects designs with a random probability based on fitness. Higher fitness individuals have a higher a probability of selection. This is often implemented using a numerical roulette wheel. Designs are allotted space on a roulette wheel in proportion to their fitness. When the wheel is spun, the designs with higher fitness have a higher probability of being selected.( see Figure 7.6)

$\begin{array}{ccc}\text { Design } & \text { Fitness } & \% \text { Total } \\ \text { A } & 40 & 12.9 \\ \text { B } & 180 & 58.1 \\ \text { C } & 90 & 29.0\end{array}$

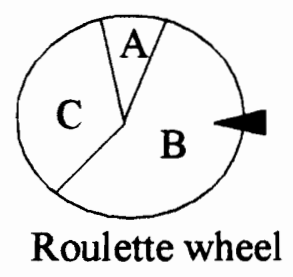

Figure 7.6 Reproduction

Crossover; Crossover is a method of swapping the coded information in parent strings to form new strings or children. This requires that two parent genetic strings are selected using the selection operator. With 
single point crossover, the coded information is swapped between strings by cutting both strings at the same point, and exchanging the coded information on one side of the cut.(see Figure 7.7). Crossover is usually applied with a high probability, with rates between $60 \%$ and $80 \%$ yielding satisfactory results on most problems ${ }^{[70]}$ Multiple crossover points are sometimes used with this operator. When crossover is not applied, the selected parents become child designs and are copied into the next generation.

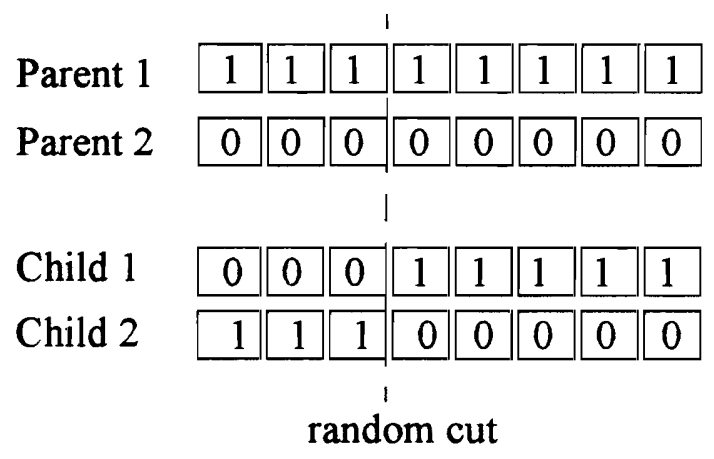

Figure 7.7 Crossover

Mutation Mutation randomly selects a gene, and randomly changes the allele with a random probability (probability of mutation). This operator introduces new information or reintroduces information that might have been prematurely discarded.

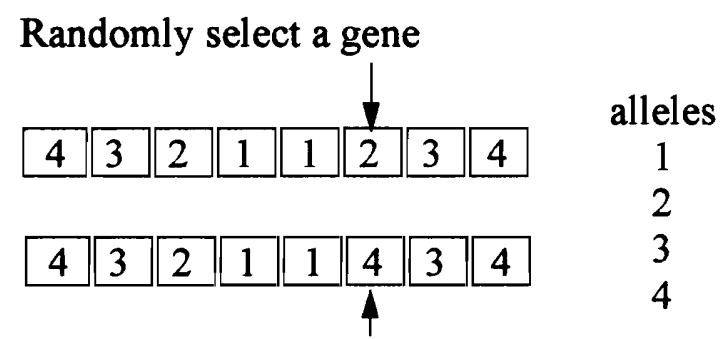

Change the allele randomly with a given probability

Figure 7.8 Mutation 
There is a trade-off between exploration of the domain, and exploitation of the fitness information present in the current generation. Reproduction exploits information about fitness such that schema ${ }^{[68]}$, (string-subsets) which contribute to high fitness are more likely to be passed on to the next generation. The randomness present in crossover and mutation explores the design space so that convergence to a global optimum is more likely. For example, exploitation can be increased by scaling fitness, giving more weight to the best individuals. Increasing the probabilities of mutation, and crossover will increase exploration. Increasing population size also increases exploration.

Constraints are implemented primarily through penalty functions. Penalties reduce the fitness of an infeasible design. This reduces the probability that infeasible designs will be selected by the reproduction operator. There is often a delicate balance when using penalties. If a penalty is too large, convergence will be adversely affected because exploration of the infeasible design space will be precluded, If a penalty is too small, infeasible designs will be produced. Conventional wisdom is that a penalty should be proportional to the magnitude of constraint violation ${ }^{[76]}$. Tuning of penalty functions is to an extent problem specific, and one of the most troublesome aspects of optimization by genetic algorithms.

The genetic algorithm will make rapid improvement in the early generations, but the rate of improvement will decline as the algorithm evolves. Eventually, many generations will pass with no improvement in the objective function. This condition is used as a convergence criterion. A number of generations " $\mathrm{N}$ " is selected, and the algorithm is stopped after $\mathrm{N}$ generations have passed with no improvement in the objective.

Among the more specialized techniques of genetic optimization are elitism ${ }^{[7]}$, and the No twins rule. Elitism preserves the best individual from each generation by a direct copy into the next generation. This insures continuous progression towards an optimum, with no backward motion, but is not guaranteed to increase the rate of convergence. The "no twins 
rule" requires that each individual in a generation is unique. It sometimes occurs that twins are produced by the reproduction operators. Since it is expensive to evaluate identical designs in the same generation, the rule requires that one of the twins is modified to make it unique.

\subsection{ALGORITHM IMPLEMENTATION}

Algorithm implementation variables affecting performance are coding, crossover rate, mutation rate, population size, and fitness and penalty formulation. Each of these variables can affect the cost and reliability of the optimization analysis. This section discusses how each performance variable was approached and established in the present work.

\subsubsection{Genetic coding for laminate design}

For optimization of energy absorption, the genetic algorithm was initially implemented using a coded representation of laminate stacking sequence only. The coding for shape optimization variables will be introduced in a later section. A discrete number of ply orientations are chosen, and then each orientation is assigned an integer code value as shown in Table 1.

Table 7.1 Ply Codes for laminate optimization

\begin{tabular}{cc}
\hline \hline Integer Ply Code & Orientation Angle (degrees) \\
\hline 1 & 0 \\
2 & +30 \\
3 & -30 \\
4 & +45 \\
5 & -45 \\
6 & +60 \\
7 & -60 \\
8 & 90 \\
\hline \hline
\end{tabular}


With this coding, a laminate stacking sequence can be represented as a unique string of positive integers. For example, an 8 ply symmetric laminate which would normally be represented as $[+45 /-45 / 0 / 90]_{\mathrm{S}}$, would be written as $[4,5,1,8]_{\mathrm{S}}$ using the coding from Table 1 . The present frame analysis can potentially have many independent laminates as design variables. When more than one laminate is being optimized, the strings for each laminate are joined to form one longer string. Coding allows the initial population of laminate stacking sequences to be generated as a series of random integer numbers, and simplifies implementation of the genetic algorithm by isolating the "problem specific" features. Problem specific features such as ply orientation are represented by the coding. The representation of these coded strings can change from problem to problem, without requiring changes in the basic operations of the genetic algorithm.

The coding alphabet in Table 7.1 is the probably the most natural for stacking sequence optimization because there is a direct relation between the number of plies, and the number of alleles. This coding is only one of several possibilities. A three digit binary representation of each ply is the next most logical choice. With respect to the choosing a coding for genetic algorithms, Goldberg ( Ref [ 69 ] page 80) offers the following principles;

1) Principle of meaningful building blocks: "The user should select a coding so that short, low-order schemata are relevant to the underlying problem and relatively unrelated to schemata over other fixed positions."

2) Principle of minimal alphabets: "The user should select the smallest alphabet that permits a natural expression of the problem."

For the coding of Table 7.1, meaningful blocks are of order one, and are unrelated to other fixed positions. Thus the requirements of the first principle are satisfied. The second principle suggests that the binary coding would be more efficient since it reduces the alphabet from 8 , to 2 . But there is a trade-off since the order of a meaningful schemata is increased 
from 1 to 3 . LeRiche ${ }^{[75]}$ used laminate codings with 3 digit alphabets and demonstrated a slight performance improvement over the binary equivalent. Preliminary tests on the plate bending test problem showed promising results so the more natural coding of Table 7.1 was retained for the energy optimization problem.

\subsubsection{Establishing population size and probability of mutation}

The computational cost of the present progressive failure analysis is too high to permit optimizing of the genetic algorithms performance directly. As an alternative, a smaller problem with the same design space, and a very inexpensive fitness evaluation was used for rough estimates of the best population size, and probability of mutation to use with the present optimization problem. The inexpensive problem involved maximizing the displacement at the center of a flat plate in bending subject to a "no failure" constraint. This problem provided some guidelines for implementation of the genetic algorithm on the current problem of optimization for energy absorption.

Reliability is the probability that a design produced by the genetic algorithm will have the global optimum fitness, and normalized cost is the number of evaluations of the objective function divided by the reliability These measures of performance were used to establish the values for population size, and probability of mutation in the present work.

Recall that mutation is a genetic operator that randomly changes the coded number in a single gene, from one allowable allele to another. The frequency at which this occurs is referred to as the mutation rate, or probability of mutation. To estimate the optimal mutation rate, the plate bending problem was used for analysis and the probability of mutation was varied from $1 \%$, to $25 \%$, in $1 \%$ increments. The laminate coding was as given in section 7.4.1. At each percent variation in probability of mutation, 50 optimization analysis were performed, and the average reliability, and average cost were calculated. A moving average of the output data for reliability is shown in Figure 7.9, and likewise for normal- 
ized cost in Figure 7.10. The moving average (width $=3$ ) was used to smooth out random variations resulting from the relatively low number of analysis used at each percent variation.

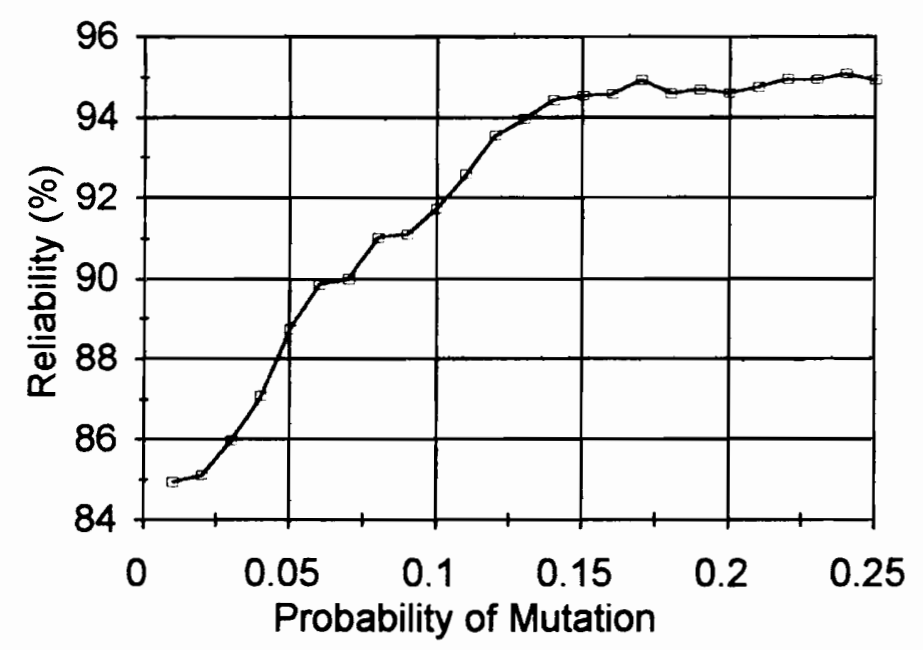

Figure 7.9 Probability of Mutation.vs. Reliability

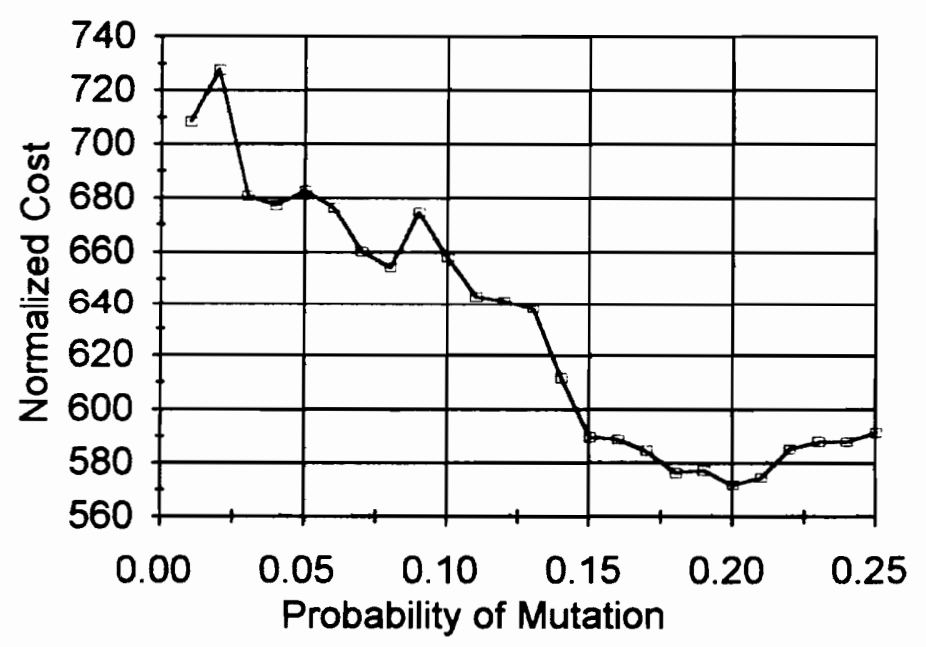

Figure 7.10 Probability of Mutation .vs. Normalized cost.

Based on the analysis results presented in Figure 7.9, and Figure 7.10, the probability of mutation was set at $15 \%$. At this value, the increase in reliability with respect to an 
increase in probability of mutation, appears to level off, as does the reduction in normalized cost.

The population size also affects cost and reliability of the genetic algorithm. A population that is too small will not have the necessary "genetic diversity", and may tend to converge early to a nonoptimal solution. A population that is too large leads to excessive computation. The number of individuals in the population remains constant throughout the optimization process. The best population size for a particular problem is influenced by the number of genes, and the number of allowable values (alleles) for each gene in the genetic string. As was done for the probability of mutation, the most efficient population size can be estimated using measures of reliability and cost. In this case, the population size for the plate bending problem was varied from 6 individuals to 12 individuals, and at each increment, 50 optimization analysis were performed. Again, the average normalized cost and reliability for each population size was calculated. Moving averages for reliability, and normalized cost verses population size are shown in Figure 7.11, and Figure 7.12, respectively.

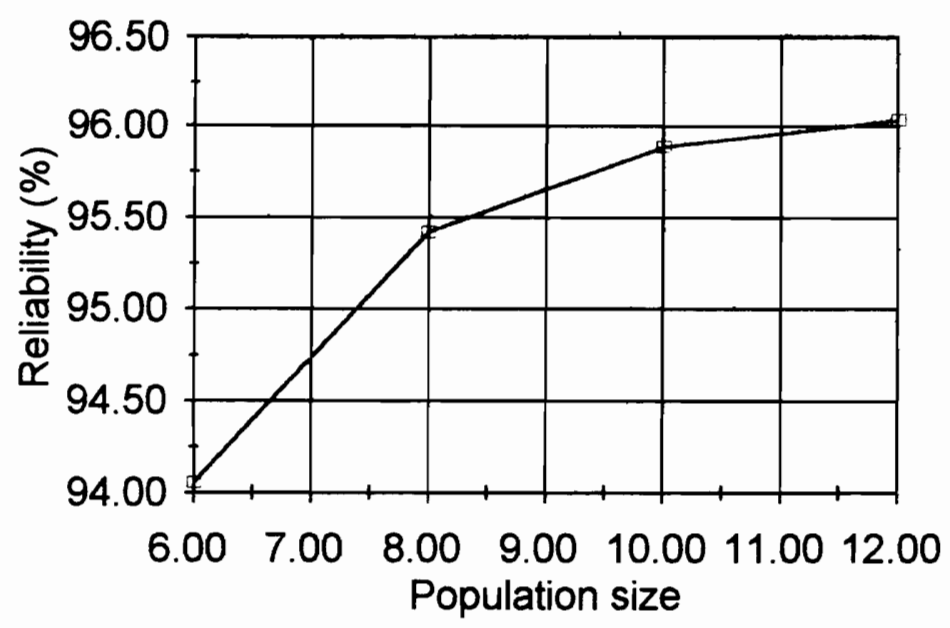

Figure 7.11 Population size .vs.reliability 


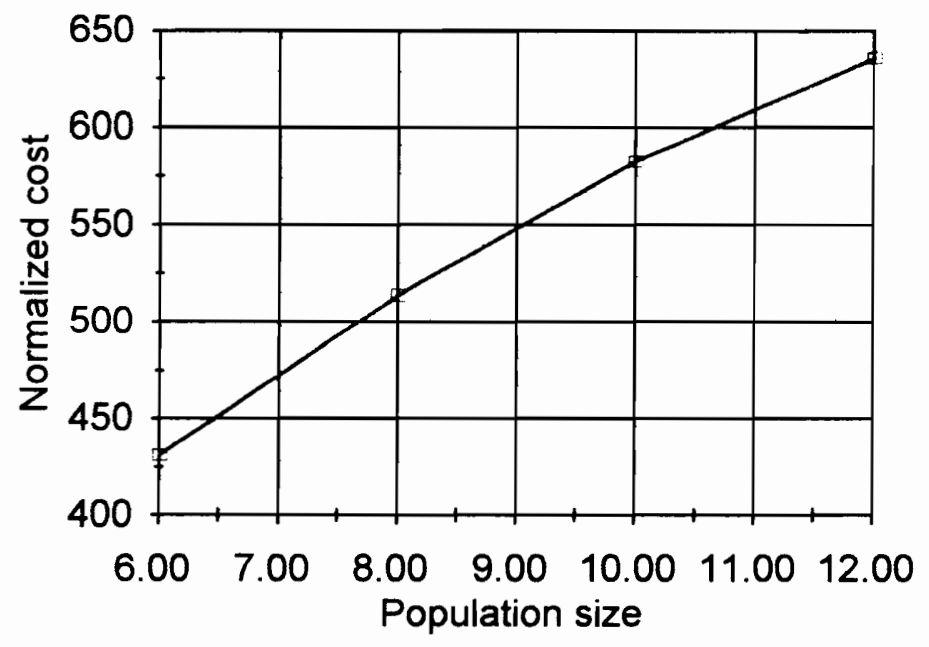

Figure 7.12 Population size .vs.cost

A population size of 10 individuals was selected based on the results presented in Figure 7.11, and Figure 7.12. At this population size, the reliability appears to level off, and further increases in population size are less rewarding. Since the cost continues to increase with increasing population size, 10 individuals is a good choice. The low normalized costs for the population sizes less than 10 , are gained at the expense of lower reliability.This choice for population size is supported by the work of Le Riche ${ }^{[75]}$ who obtained a population size of 8 for a similar problem involving stacking sequence optimization for plate buckling.

\subsubsection{Crossover rate and convergence.}

There are two additional performance parameters which must be established. These are the crossover rate, and the convergence criterion. The crossover rate was set at $75 \%$ based on the range of values known to give satisfactory results, and single point crossover was used. The convergence criterion establishes a stopping point for the optimization. The genetic algorithm is allowed to evolve until a pre-specified number of generations have 
passed with no improvement in the objective function. This parameter is more problem specific, and the convergence was initially set relatively low at 25 generations without improvement due to the high cost of each progressive failure analysis. On the IBMRS6000, each analysis of a generation (10 individuals ) requires an average of approximately 2 minutes of clock time when the system load is light. Each progressive failure analysis consists of an indeterminate number of frame analysis, estimated at an average of 20 or more frame analysis per progressive failure analysis. This translates into roughly 12 seconds per progressive failure analysis, and 0.6 seconds for an average frame analysis and modification cycle. Thus even though the beam analysis is considered efficient, each 30 generations of 10 individuals will require approximately 1 hour for analysis. For the initial frame problems with a small design space, the low convergence criterion of 25 generations appears to produce near optimal designs a high percentage of the time. This will be explained more fully as the examples are presented. As the design space increased with the addition of shape variables and additional laminates, the convergence criterion was increased to 100 generations. The computational requirements of the analysis limit the type of reliability studies which are often performed with genetic optimization methods.

\subsubsection{Fitness calculation}

The limit on maximum crushing force is implemented as a penalty. A symmetry condition on stacking sequence was imposed by creating only half of the laminate randomly, and copying those plies to the other half as a mirror image.

The raw fitness of each design is evaluated with a function of the form given by,

$$
\text { Raw fitness }=f=E_{a}\left(1-p_{1}\right),
$$

where " $E_{\mathrm{a}}$ " is the absorbed energy given by the total area under the load displacement curve Eq. ( 7.1 ), and $p_{1}$ is a penalty for exceeding the maximum allowable crushing force. 
Penalty "p." was set to 0.75 if the maximum force was exceeded, or zero otherwise. This penalty was determined by increasing " $p_{1}$ " until infeasible designs no longer appeared. A very stiff and strong frame design absorbs a lot of energy, but may violate the maximum failure constraint by 4 times or more. The penalty must be very high to lower these super individuals chance for selection. The form of Eq. (7.2) is probably not a good choice because the penalty is large, and is not proportional to the constraint violation. An improved penalty will be discussed in a later section.

After ranking ${ }^{1}$, the raw fitness of Eq. ( 7.2 ) is modified to prevent high fitness individuals from dominating in the early stages of optimization, and to increase the selection pressure in the later stages. In the early generations, schema from extremely high fitness individuals may take over, and in this case the algorithm will converge early. In the later generations, average fitness is higher, and there is less bias in the selection process towards the highest fitness individuals. To solve these problems, modification of the raw fitness is frequently done using linear scaling. Linear scaling is illustrated in Figure 7.13 and Eq.( 7.3 )

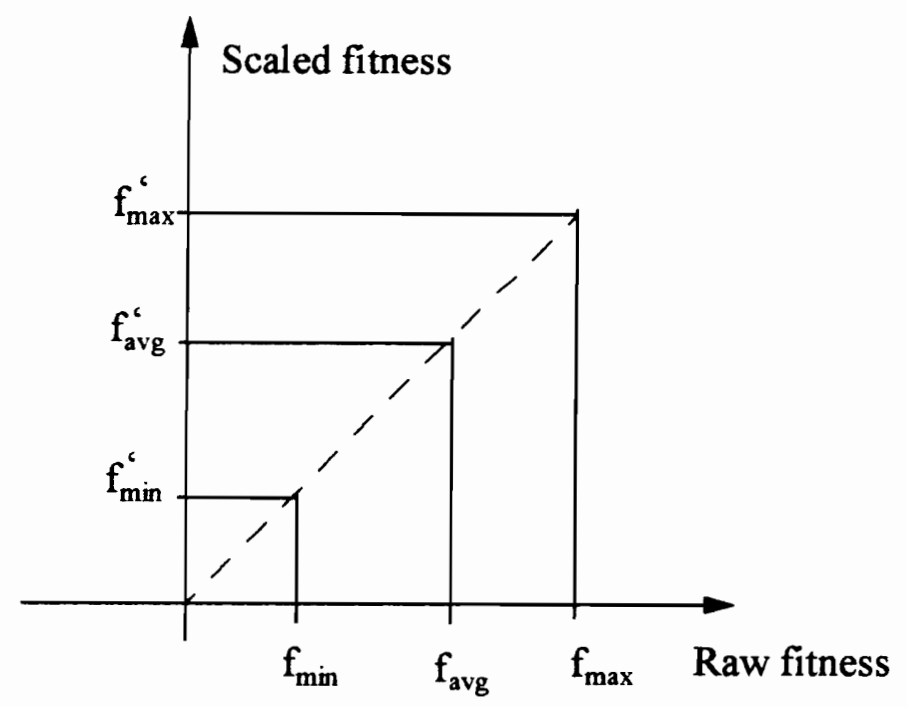

Figure 7.13 Fitness scaling

1. Ranking refers to sorting the individuals of a generation according to their fitness. 


$$
\begin{aligned}
\text { Scaled fitness }=\mathrm{f} & =\mathrm{af}+\mathrm{b} \\
\mathrm{a} & =0.5\left(\mathrm{f}_{\mathrm{avg}} /\left(\mathrm{f}_{\max }-\mathrm{f}_{\mathrm{avg}}\right)\right) \\
\mathrm{b} & =\mathrm{f}_{\mathrm{avg}}\left(\left(\mathrm{f}_{\max }-1.5 \mathrm{f}_{\mathrm{avg}}\right) /\left(\mathrm{f}_{\max }-\mathrm{f}_{\mathrm{avg}}\right)\right)
\end{aligned}
$$

where in Eq. ( 7.3), the scaled maximum fitness $\mathrm{f}_{\max }$ is $1.5 \times \mathrm{f}_{\mathrm{avg}}$.

The two special strategies discussed earlier were implemented with the present genetic algorithm. One, the elitist method, which ensures that the best design is carried to the next generation, and two, the "no twins" rule, which guarantees that each generation is composed of unique individuals.

\subsubsection{Genetic Optimization of Energy Absorption}

The flow of genetic optimization for energy absorption is illustrated by Figure 7.14. After pre-processing, the analysis begins with the random generation of " $N$ " genetic strings of the proper length, and with valid alleles in each gene. Each string is a coded representation of the composite frame design variables (the specific coding will be discussed later). Using the conventional terminology, each string is referred to as an individual. A loop over each individual in the population ( from $\mathrm{I}=1$ to $\mathrm{N}$ ), calculates the fitness for each design. As shown in Figure 7.14, this loop contains three operations. First, the coded string must be translated into a structural design. The string represents elements of the frame that are design variables for optimization, not the entire design. In the program produced as part of this work, the frame design (nodal geometry, boundary conditions, element connectivity, etc ) is read in, processed, and stored as part of the "pre-processing" operation. This moves a portion of the work out of the optimization loop, and increases efficiency. The coded design string is then interpreted as changes to the baseline design. Second, the progressive failure analysis is applied. This gives the value of the objective Eq. ( 7.1 ). Third, the constraint penalties are calculated and applied to obtain a fitness for 
the current individual. When this loop is complete the fitness for each individual is available to be ranked from lowest to highest and scaled. If the convergence criterion is met, the optimization stops, otherwise, the reproduction operators are applied. This creates a new generation of individuals and the process continues until convergence is obtained.

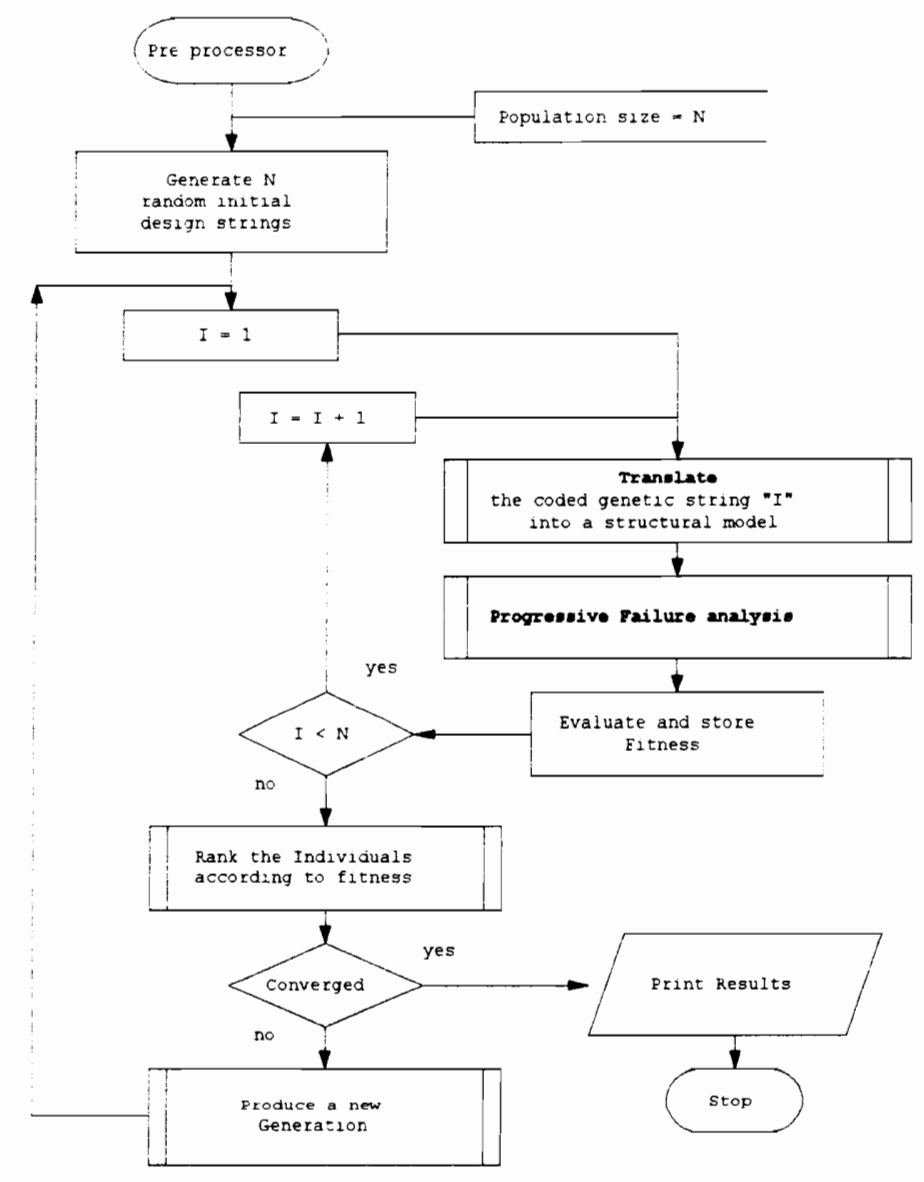

Figure 7.14 Flow diagram of optimal design using Genetic Algorithms

\subsection{OPTIMIZATION RESULTS AND DISCUSSION}

\subsubsection{Laminate optimization}

The optimization methodology will be demonstrated using several examples. The frame example configuration is a semi-circular frame with a 36 inch radius of curvature. 
Clamped end conditions are assumed and the load is applied radially inward at the apex. A uniform cross section is assumed along the circumference of the frame, and the same laminate is used for each segment of the cross section. This laminate is arbitrarily set to a constant thickness of eight plies. Ply orientation, and stacking sequence are the design variables. These ply orientations are limited to the eight discrete values given in Table 7.1 $(0,+30,-30,+45,-45,+60,-60$, and 90 degrees). A constraint is placed on the maximum allowable crushing force, and laminate symmetry is enforced. The size of the design space for this example is $8^{4}$ or 4096 feasible design configurations. The geometry for the first example problem is shown in Figure 7.15.
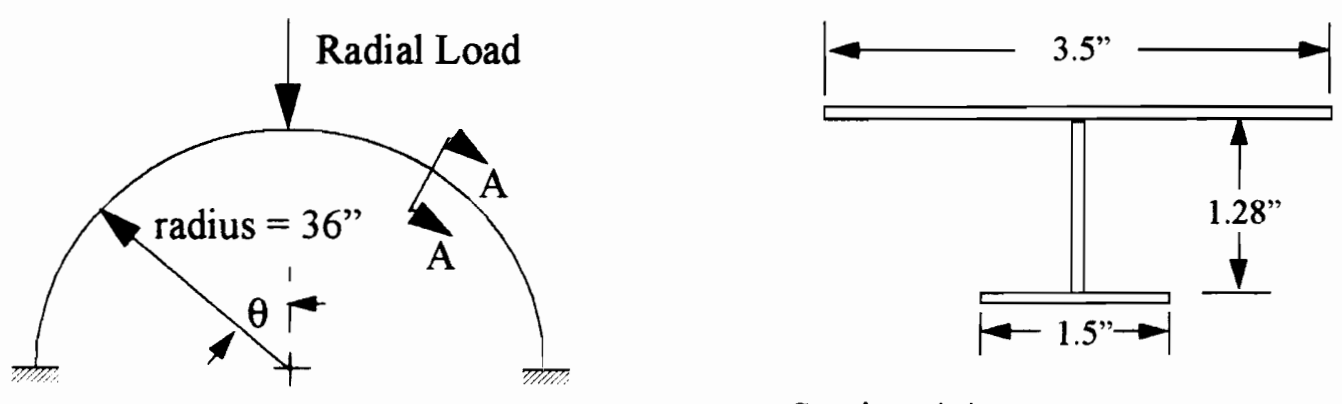

Section AA

Figure 7.15 Cross section geometry for example problems.

Material for this example frame is AS4/5208, graphite epoxy $\left(\mathrm{E}_{1}=18.4 \times 10^{6} \mathrm{psi}, \mathrm{E}_{2}=\right.$ $\left.1.64 \times 10^{6} \mathrm{psi}, \mathrm{G}_{12}=.87 \times 10^{6} \mathrm{psi}, \mathrm{v}_{12}=.30, \mathrm{t}=.00525^{\prime \prime}\right)$. The strength data was taken as, $X=197.9 \times 10^{3} \mathrm{psi}, X^{\prime}=160.0 \times 10^{3} \mathrm{psi}, \mathrm{Y}=5.5 \times 10^{3} \mathrm{psi}, \mathrm{Y}^{\prime}=29.9 \times 10^{3} \mathrm{psi}, \mathrm{S}=14.0 \times 10^{3} \mathrm{psi}$, and the interaction term $\mathrm{F}_{\mathrm{xy}}^{*}$ was taken as -0.5 .

Two composite fuselage frames are designed for crashworthiness, and compared to a baseline composite frame. The first frame is designed to achieve a $500 \mathrm{lb}$ crush load, and the second is designed for a $1000 \mathrm{lb}$ crush load. The baseline case is a quasi-isotropic wall lay up. 
Load displacement response diagrams from optimizations at the two different crushing loads are shown in Figure 7.16, and Figure 7.17. The failure sequence for both frames is similar. Failure begins near the point of load application on the radially inboard flanges of the frame. The weakest plies fail, then failure progresses to inner flanges at the fixed ends ( \pm 90 degrees ). These are both tensile failures due to bending of the cross section. The process continues by alternating between these two locations until ultimate failure occurs. The total energy absorbed and the optimal laminate stacking sequences obtained are summarized in Table 7.2

Table 7.2 Frame optimization example \#I

\begin{tabular}{ccc}
\hline \hline $\begin{array}{c}\text { Design case } \\
\text { lbs }\end{array}$ & $\begin{array}{c}\text { Energy Absorbed } \\
\text { (in*lbs) }\end{array}$ & $\begin{array}{c}\text { Optimal Stacking } \\
\text { Sequence }\end{array}$ \\
\hline 500 & 422.8 & {$[60 / 90 / 45 /-45]_{\mathrm{s}}$} \\
1000 & 913.5 & {$[-30 / 45 / 90 /-45]_{\mathrm{s}}$} \\
\hline
\end{tabular}

The optimal solutions were obtained relatively quickly. For the $500 \mathrm{lb}$ case, the optimal solution required 52 generations. This is a total of 520 progressive failure analysis (recall that 10 individuals were used in each generation), which represents approximately $12.6 \%$ of the design space. For the $1000 \mathrm{lb}$ case, 43 generations were required, which represents $10.4 \%$ of the design space.

For both load cases, designs were obtained which did not violate maximum crushing force constraint. The crushing load approached the limit, but remained just below it as a sus- 
tained force until ultimate failure. This behavior is precisely what we had hoped to achieve

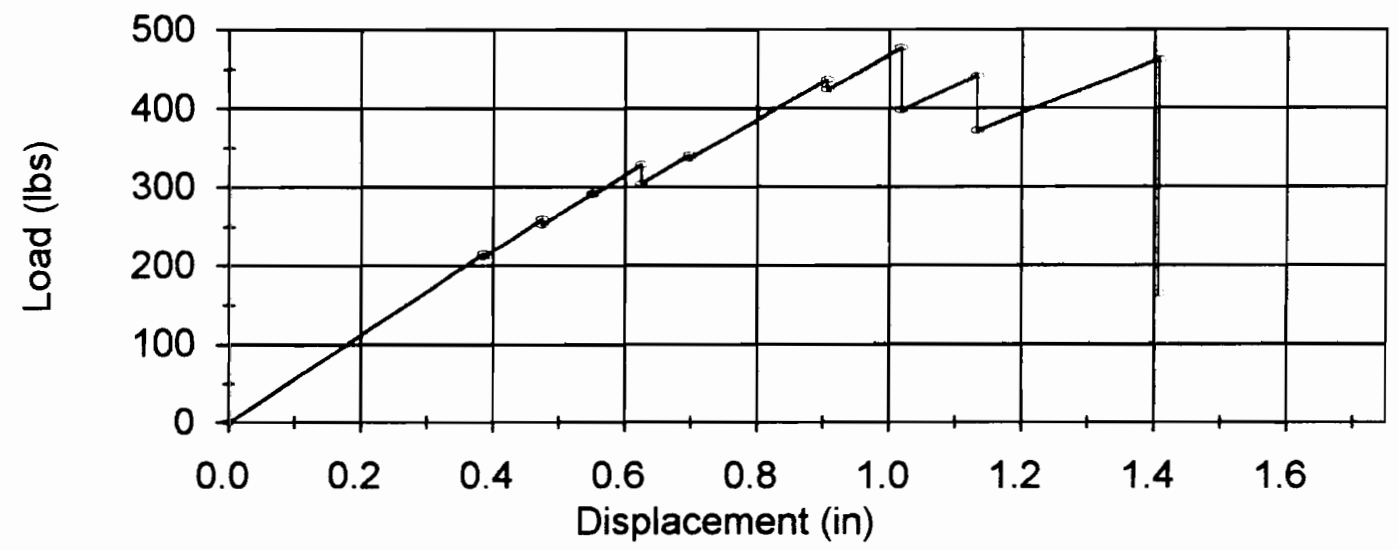

Figure 7.16 Frame response optimized for $500 \mathrm{lb}$ crush response.

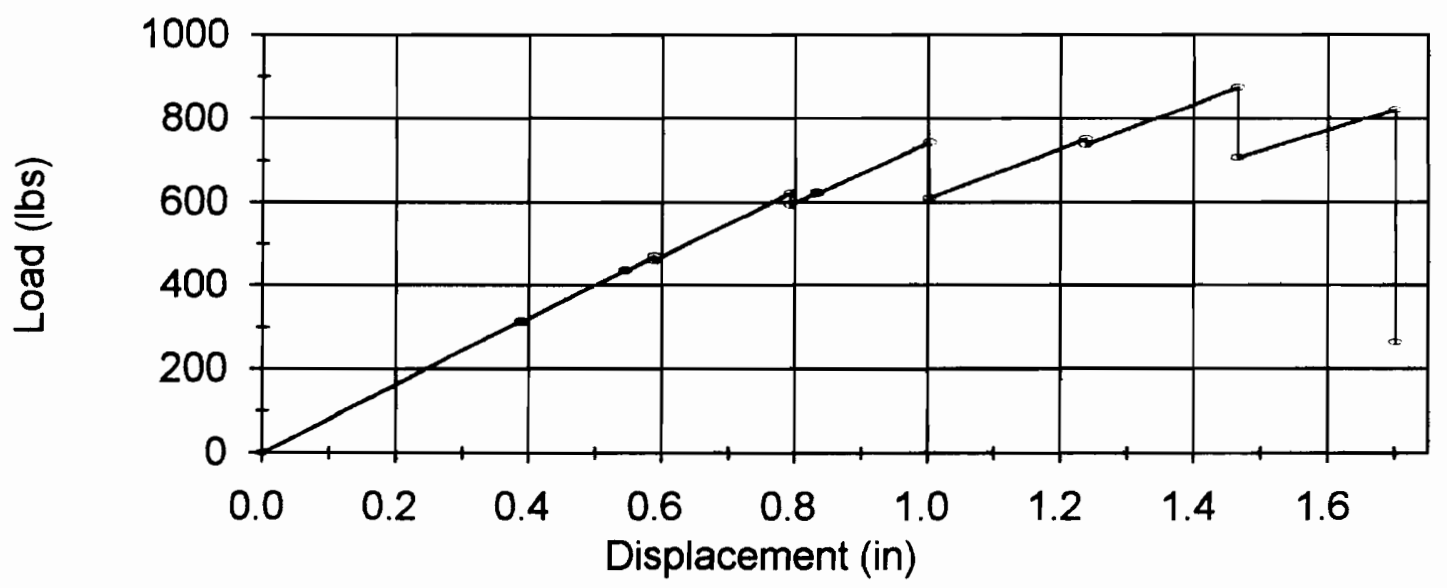

Figure 7.17 Frame response optimized for $1000 \mathrm{lb}$ crush force

A comparison to progressive failure analysis results for a quasi isotropic [ $45 /-45 / 0 / 90$ ]$_{\mathrm{S}}$ frame is shown in Figure 7.18. This quasi isotropic frame represents a composite design which has not been optimized for crashworthiness, but could be used for reduced weight (Note that all three frames represented have the same weight). The maximum crushing load in this case reaches $1392 \mathrm{lbs}$, and the total energy absorbed prior to ultimate failure is 
816.4 in*lbs. At the first limit load level of $500 \mathrm{lbs}$, the quasi isotropic frame has absorbed approximately 98 in*lbs of crash energy. This represents less than $24 \%$ of the energy absorbed by the frame optimized for the $500 \mathrm{lb}$ crush load. At the $1000 \mathrm{lb}$ limit load, the quasi isotropic frame has absorbed approximately 405 in*lbs of energy, or less that $45 \%$ of the energy absorbed by the frame optimized for that load level. It is important to remember that for survivability, the crash energy must be absorbed before the occupants are exposed to lethal levels of acceleration. For both design cases, the optimized frames would significantly reduce the peak acceleration loads on the vehicle occupants

Table 7.3 Design improvement over baseline

\begin{tabular}{cccc}
\hline \hline $\begin{array}{c}\text { Design load case } \\
\text { (lbs) }\end{array}$ & $\begin{array}{c}\text { Energy absorbed } \\
\text { (in*lbs) }\end{array}$ & $\begin{array}{c}\text { Baseline energy } \\
\text { (in*lbs) }\end{array}$ & \% improvement \\
\hline 500 & 422.8 & 98.0 & 331.0 \\
1000 & 913.5 & 405.0 & 125.0 \\
\hline \hline
\end{tabular}

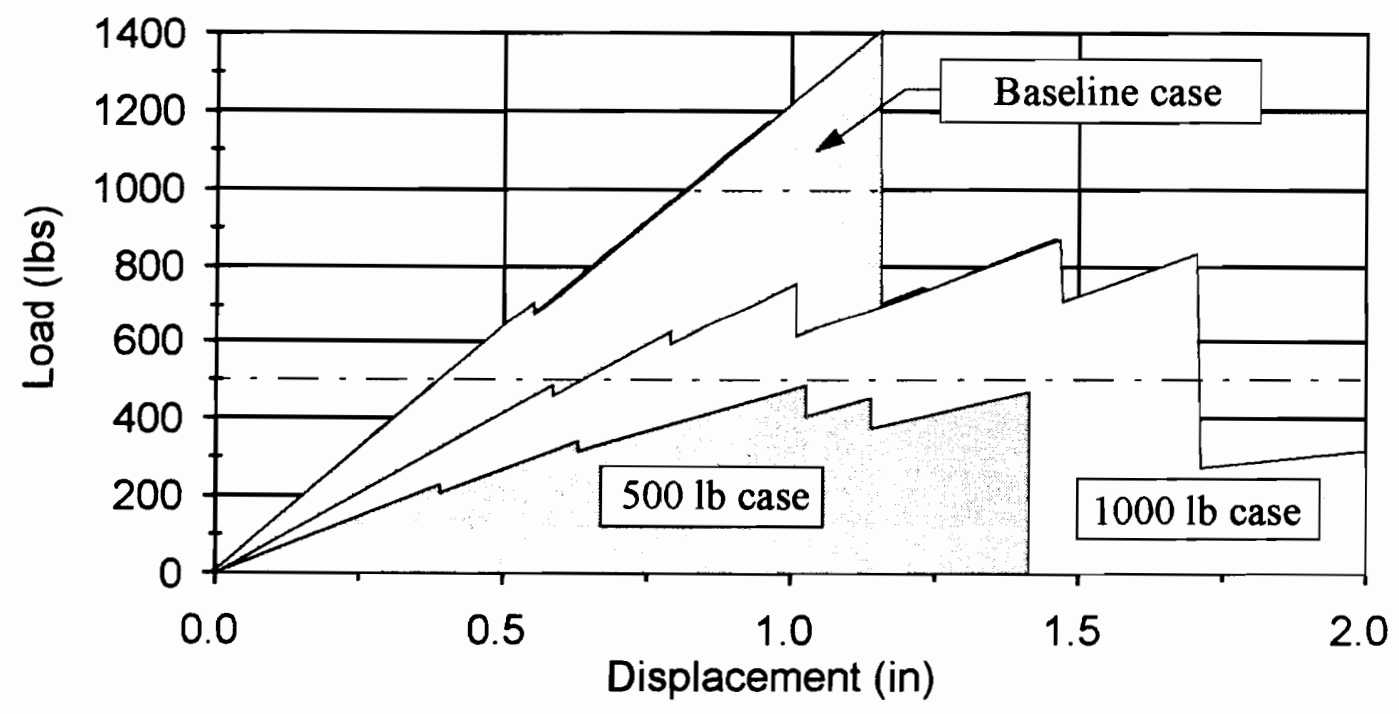

Figure 7.18 Comparison to quasi isotropic design case.

Both the $500 \mathrm{lb}$ load case, and the $1000 \mathrm{lb}$ load case were repeated to verify results of the optimization. In both cases, the original optimal designs as described in Table 7.2, were 
repeated. In addition, for the $500 \mathrm{lb}$ load case, 4 additional designs were found with approximately equivalent energy absorption to the $500 \mathrm{lb}$ case of Table 7.2. The laminate stacking sequences for these frames are given in Table 7.4. For the $1000 \mathrm{lb}$ load case, one additional design was found as listed in Table 7.5.

Table 7.4 Additional $500 \mathrm{lb}$ designs for example \#1

\begin{tabular}{cc}
\hline \multicolumn{2}{c}{$500 \mathrm{lb}$ Load case } \\
$\begin{array}{c}\text { Energy Absorbed } \\
\text { (in*lbs) }\end{array}$ & $\begin{array}{c}\text { Optimal Stacking } \\
\text { Sequence }\end{array}$ \\
\hline 417.5 & {$[45 / 60 /-45 / 90]_{\mathrm{S}}$} \\
422.8 & {$[90 / 60 / 45 /-45]_{\mathrm{S}}$} \\
422.8 & {$[-60 / 90 /-45 / 45]_{\mathrm{S}}$} \\
422.8 & {$[90 / 60 /-45 / 45]_{\mathrm{S}}$} \\
\hline
\end{tabular}

Table 7.5 Additional $1000 \mathrm{lb}$ designs for example \#I

\begin{tabular}{cc}
\hline \multicolumn{2}{c}{$1000 \mathrm{lb}$ Load case } \\
$\begin{array}{c}\text { Energy Absorbed } \\
\text { (in*lbs) }\end{array}$ & $\begin{array}{c}\text { Optimal Stacking } \\
\text { Sequence }\end{array}$ \\
\hline 881.6 & {$[90 /-45 / 60 / 30]_{\mathrm{S}}$} \\
\hline \hline
\end{tabular}

\subsubsection{Cross-sectional geometry optimization}

Next, cross section shape variables were added to the design vector. Shape optimization was approached as discrete variations from a baseline cross section. This approach allows the shape optimization to be treated by the genetic algorithm in a similar way to the laminate design.

The method will be illustrated with an example. Recall from Chapter 3, that the curved beam cross section is described using cross section nodes connected by straight line branches (laminates). Nodal input for the example cross-section illustrated in Figure 7.19, is listed in Table 7.6. Nodal coordinates $\mathrm{Y}$, and $\mathrm{Z}$ determine the shape of the cross section. 
Changes in these nodal coordinates are defined by parameters $Y_{\mathcal{C}}$ and $Z_{c}$, which determine how the shape can change during optimization. In the example data of Table 7.6, the negative three in column $\mathrm{Y}_{\mathrm{c}}$ for node one (row 1), constrains the $\mathrm{Y}$ motion of node one to be the opposite of the node three $\mathrm{Y}$ motion. (here motion refers to the change in section shape during optimization.). The three in column $\mathrm{Y}_{\mathrm{C}}$ of node three (row 3 ), indicates that this is an independent design variable. In general, when the absolute value of $Y_{c}$ or $Z_{c}$ equals the row number of the node, then this nodal degree of freedom is an independent design variable and must be added to the genetic string. For the example section, the $\mathrm{Y}$ degree of freedom for node one is constrained to move opposite of node three, and likewise for nodes 4 , and 6 respectively. The remaining Y-degrees of freedom are fixed. The Z-degrees of freedom for nodes one, two, and three, are constrained to move together, with degree of freedom $Z(3)$, and the remaining $Z$-degrees of freedom are fixed The three independent design variables for this example control motion of $\mathrm{Y}(3), \mathrm{Y}(6)$, and $\mathrm{Z}(3)$ nodal degrees of freedom.

Table 7.6 Example of nodal input data for cross section shape.

\begin{tabular}{ccccc}
\hline \hline Node \# $=\mathrm{i}$ & $\mathrm{Y}(\mathrm{i})$ & $\mathrm{Z}(\mathrm{i})$ & $\mathrm{Y}_{\mathrm{d}}(\mathrm{i})$ & $\mathrm{Z}_{\mathrm{d}}(\mathrm{i})$ \\
\hline 1 & -1.0 & 2.0 & -3 & 3 \\
2 & 0.0 & 2.0 & 0 & 3 \\
3 & 1.0 & 2.0 & 3 & 3 \\
4 & -1.0 & 0.0 & -6 & 0 \\
5 & 0.0 & 0.0 & 0 & 0 \\
6 & 1.0 & 0.0 & 6 & 0 \\
\hline \hline
\end{tabular}




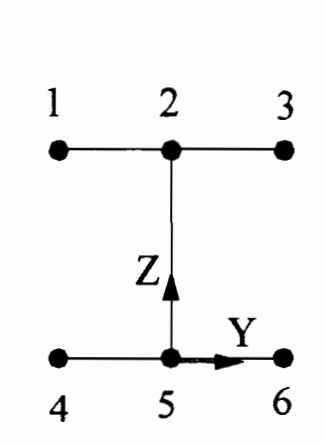

Node Numbering

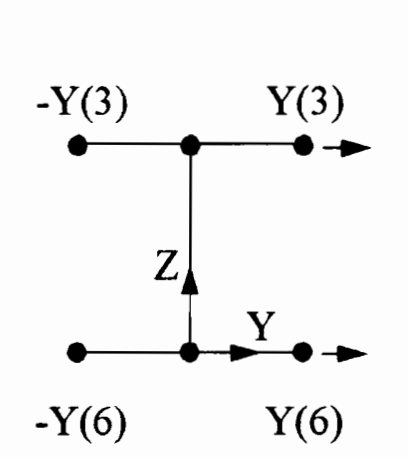

Y-degrees of freedom

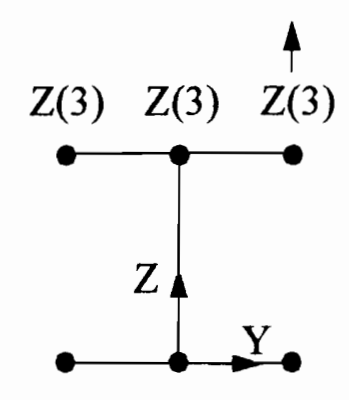

Z-degrees of freedom

Figure 7.19 Example for shape optimization.

The nodal coordinates for the moving degrees of freedom of the cross section are modified by adding a discrete delta. (e.g. $\mathrm{Y}(\mathrm{i})=\mathrm{Y}(\mathrm{i})+\Delta \mathrm{Y}(\mathrm{i})$ ). The delta for each independent degree of freedom is a unique design variable. A transformation is constructed from the input data to enforce the constrained deltas, and set the fixed deltas to zero. Constrained deltas are associated with cross-sectional nodes which are constrained to move with other nodes. Thus the cross section shape can be calculated as its baseline nodal coordinates, plus a vector of deltas for each degree of freedom.

The genetic coding for the deltas is based on a percentage variation from the baseline cross section. For the present work, seven alleles were used, with one gene per degree of freedom. This is similar to the previous coding structure used for laminates. Values of delta change in discrete increments that are set at $10 \%$ of a characteristic cross section dimension $\left(d_{c}\right)$. For the present work, this dimension was the length of the shortest ele- 
ment in the cross section. This coding is shown in Table 7.7.

Table 7.7 Shape coding

\begin{tabular}{cc}
\hline Coding & $\begin{array}{c}\% \text { Variation } \\
\left(\Delta=\mathrm{d}_{\mathrm{c}}{ }^{*}(\%) / 100\right)\end{array}$ \\
\hline 1 & -30 \\
2 & -20 \\
3 & -10 \\
4 & 0 \\
5 & 10 \\
6 & 20 \\
7 & 30 \\
\hline \hline
\end{tabular}

The alphabet is odd numbered to allow for equal chances of growth, or shrinkage, or no change with the "zero change" allele. The percent variations of the coding are easily changed, and switches are included in the present program to do this. They can be switched to all negative changes, all positive ,or mixed as shown in Table 7.7. The percentage variation can be changed as well. This allows for restarting the optimization with finer increments to further improve the design.

The $500 \mathrm{lb}$ "I" cross-section case was repeated with one laminate as before, but with three added shape variables corresponding to those used in the example. The design space for this example is significantly larger $\left(8^{4} \times 7^{3}=1,404,928\right)$. The dimensions of the baseline six noded cross section are given in Table 7.8 for reference.

Table 7.8 Baseline cross section dimensions for shape optimization

\begin{tabular}{ccc}
\hline \hline Node \# & Y(i) & Z(i) \\
\hline 1 & -0.75 & 1.3810 \\
2 & 0.0 & 1.3810 \\
3 & 0.75 & 1.3810 \\
4 & -1.75 & 0.0585 \\
5 & 0.00 & 0.0585 \\
6 & 1.75 & 0.0585 \\
\hline \hline
\end{tabular}


The Optimal design obtained for this case is given in Table 7.9, and Table 7.10, and the optimal load response is shown in Figure 7.20. The absorbed energy is increased $49.9 \%$, compared to the equivalent case with no shape optimization (Figure 7.16) The optimized cross section shape is given in Table 7.10

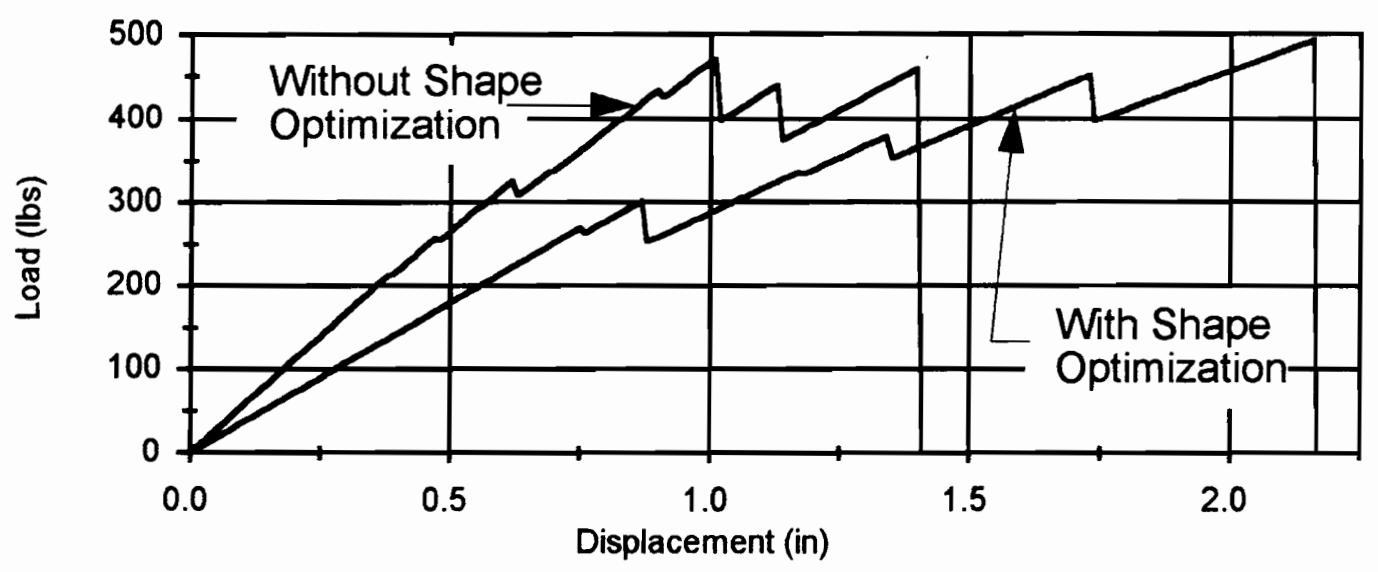

Figure 7.20 Comparison of optimized $500 \mathrm{lb}$ case with and without shape optimization

Table 7.9 Energy absorbed for the $500 \mathrm{lb}$ case with shape optimization.

\begin{tabular}{cccc}
\hline $\begin{array}{c}\text { Energy Absorbed } \\
\text { (in*lbs) }\end{array}$ & $\begin{array}{c}\text { Optimal Stacking } \\
\text { Sequence }\end{array}$ & $\begin{array}{c}\% \text { increase } \\
\text { in } \mathrm{E}_{\mathrm{a}}\end{array}$ & $\begin{array}{c}\text { \% increase from } \\
\text { baseline } \mathrm{E}_{\mathrm{a}}\end{array}$ \\
\hline 633.151 & {$[-45 / 45 / 45 /-60]_{\mathrm{s}}$} & 49.9 & 546.07 \\
\hline
\end{tabular}

a. \% Increase over previous $500 \mathrm{lb}$ case with laminate optimization only(Table 7.3)

b. \% Increase over baseline quasi-isotropic (See Table 7.3) 
Table 7.10 Optimal cross section dimensions for the $500 \mathrm{lb}$ case, with shape optimization.

\begin{tabular}{ccc}
\hline \hline Node \# & Y(i) & Z(i) \\
\hline 1 & -0.90 & 1.1560 \\
2 & 0.0 & 1.1560 \\
3 & 0.90 & 1.1560 \\
4 & -1.60 & 0.0585 \\
5 & 0.00 & 0.0585 \\
6 & 1.60 & 0.0585 \\
\hline \hline
\end{tabular}

As shown by the data in Table 7.10, the height of the cross section was reduced, and the lengths of the radially outboard flanges were also reduced, The radially inboard flange length was increased. Additional optimization runs with this same configuration produced similar designs with approximately the same energy absorption. Three are listed in Table 7.11

Table 7.11 Optimal designs obtained with shape optimization

\begin{tabular}{ccccc}
\hline \hline $\begin{array}{c}\text { Energy Absorbed } \\
\text { (in*lbs) }\end{array}$ & Stacking Sequence & $\mathrm{Y}(3)$ & $\mathrm{Y}(6)$ & $\mathrm{Z}(3)$ \\
\hline 629.6 & {$[45 / 45 /-45 /-60]_{\mathrm{s}}$} & 0.90 & 1.525 & 1.156 \\
632.3 & {$[-45 / 45 /-60 / 45]_{\mathrm{s}}$} & 0.90 & 1.600 & 1.156 \\
627.5 & {$[45 /-60 / 45 /-45]_{\mathrm{s}}$} & 0.90 & 1.525 & 1.156 \\
\hline \hline
\end{tabular}

\subsubsection{Separation of failure events}

Next an attempt was made to separate failure events. Failure response of the optimal designs may be sensitive to random defects. Failure events which are predicted to occur simultaneously in spatially different locations may in fact occur sequentially. The first failure will alter the loads and stiffness, and may alter the total failure sequence. To create frames in which failures do not occur simultaneously, the symmetry of the frame must be 
removed (assuming the loading is fixed). To accomplish this, the frame was divided into a right half cross-section, and a left half cross section, with section shapes that were free to vary independently in each half. This is illustrated in Figure 7.21

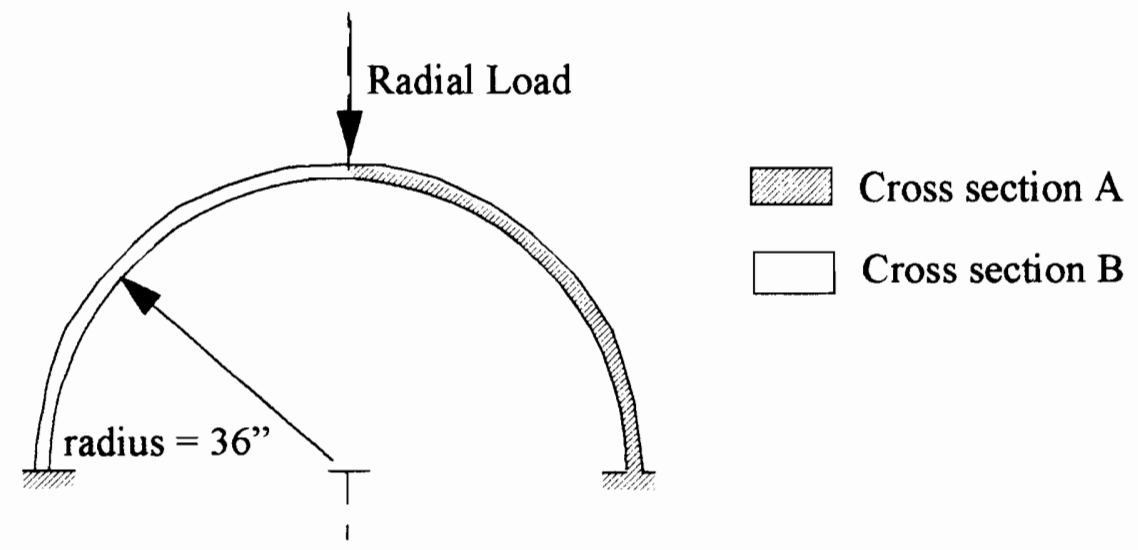

Figure 7.21 Division of cross section into two shape regions.

The design space for this example is once again significantly increased from the previous case to $8^{4} \times 7^{6}=481,890,304$ discrete possible designs. The optimal design obtained in this case described in Table 7.12, and Table 7.13, and the load response is shown in Figure 7.22

Table $7.12500 \mathrm{lb}$ case with shape optimization and failure separation

\begin{tabular}{cccc}
\hline \hline $\begin{array}{c}\text { Energy Absorbed } \\
\text { (in*lbs) }\end{array}$ & $\begin{array}{c}\text { Optimal Stacking } \\
\text { Sequence }\end{array}$ & $\begin{array}{c}\% \text { increase }^{\mathrm{a}} \\
\text { in } \mathrm{E}_{\mathrm{a}}\end{array}$ & $\begin{array}{c}\% \text { increase in } \mathrm{E}_{\mathrm{a}} \\
\text { from baseline }\end{array}$ \\
\hline 679.0 & {$[45 /-45 /-45 /-45]_{\mathrm{s}}$} & 7.26 & 592.8 \\
\hline \hline
\end{tabular}

a. \%increase from previous $500 \mathrm{lb}$ case with one laminate, and shape optimization(Table 7.9)

Table 7.13 Optimal cross section shapes

\begin{tabular}{cccccc}
\hline \hline \multicolumn{3}{c}{ Section A } & \multicolumn{3}{c}{ Section B } \\
$\mathrm{Y}(3)$ & $\mathrm{Y}(6)$ & $\mathrm{Z}(3)$ & $\mathrm{Y}(3)$ & $\mathrm{Y}(6)$ & $\mathrm{Z}(3)$ \\
\hline 0.75 & 1.60 & 1.156 & 0.825 & 1.525 & 1.156 \\
\hline \hline
\end{tabular}




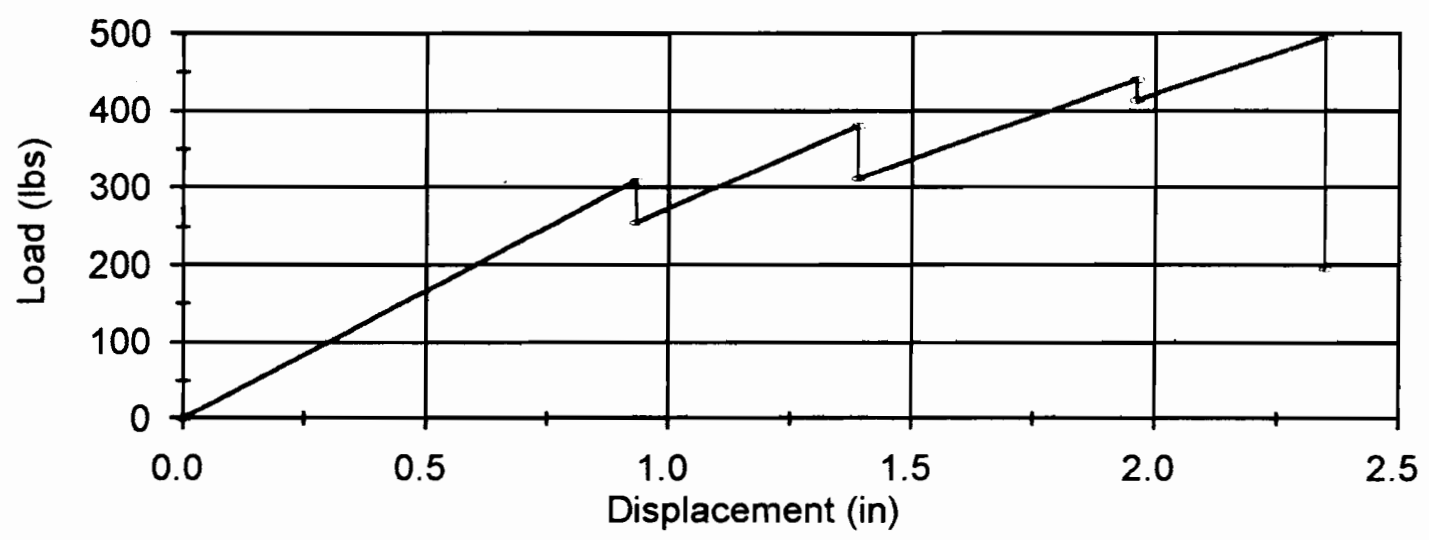

Figure 7.22 Frame response optimized for $500 \mathrm{lb}$ crush response with 2 cross sections and one stacking sequence.

As mentioned earlier, the penalty function used for these results may not allow a sufficient exploration of the infeasible design space. A new penalty function was created that is based on the area below the constraint violation on the load response diagram. This penalty is proportional to the violation of the constraint, and allows more exploration of the infeasible design space.

$$
\text { Raw fitness }=f=E_{a}-\left(C_{1} p_{2}+C_{2}\right)
$$

where $C_{1}$ is a scale factor for the constraint $p_{2}$ and $C_{2}$ is a small constant penalty that is zero if $\mathrm{p}_{2}$ equals zero, and 25 otherwise. The value used for " $\mathrm{C}_{1}$ " was 1.25 .

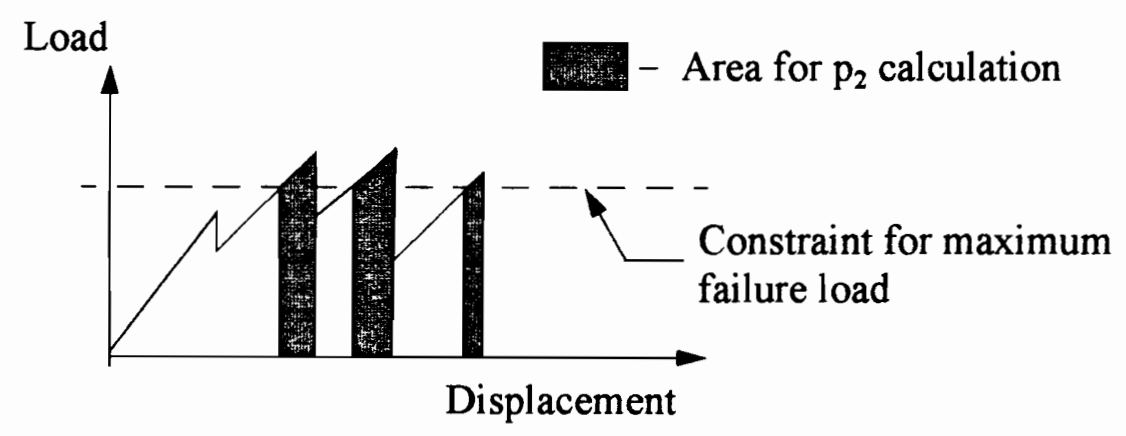

Figure 7.23 Modified Penalty constraint 
Using this new fitness and penalty, a new design was obtained which slightly improves the energy absorption This case uses two cross sections, and one laminate stacking sequence as before.

The optimal design obtained for this analysis is described in Table 7.14, and Table 7.15, and Figure 7.24.

Table 7.14500 lb case with 2 cross-sectional geometries

\begin{tabular}{cccc}
\hline \hline $\begin{array}{c}\text { Energy Absorbed } \\
\text { (in*1bs) }\end{array}$ & $\begin{array}{c}\text { Optimal Stacking } \\
\text { Sequence }\end{array}$ & $\begin{array}{c}\text { \% increase }^{\mathrm{a}} \\
\text { in } \mathrm{E}_{\mathrm{a}}\end{array}$ & $\begin{array}{c}\text { \% increase in } \mathrm{E}_{\mathrm{a}} \\
\text { from baseline }\end{array}$ \\
\hline 681.6 & {$[-45 / 45 / 45 / 45]_{\mathrm{S}}$} & 7.65 & 595.51 \\
\hline \hline
\end{tabular}

a. \%increase from $500 \mathrm{lb}$ case with one laminate, and shape optimization (Table 7.9)

Table 7.15 Optimal cross section shapes

\begin{tabular}{cccccc}
\hline \hline \multicolumn{3}{c}{ Section A } & \multicolumn{3}{c}{ Section B } \\
$\mathrm{Y}(3)$ & $\mathrm{Y}(6)$ & $\mathrm{Z}(3)$ & $\mathrm{Y}(3)$ & $\mathrm{Y}(6)$ & $\mathrm{Z}(3)$ \\
\hline 0.75 & 1.525 & 1.156 & 0.825 & 1.525 & 1.156 \\
\hline \hline
\end{tabular}

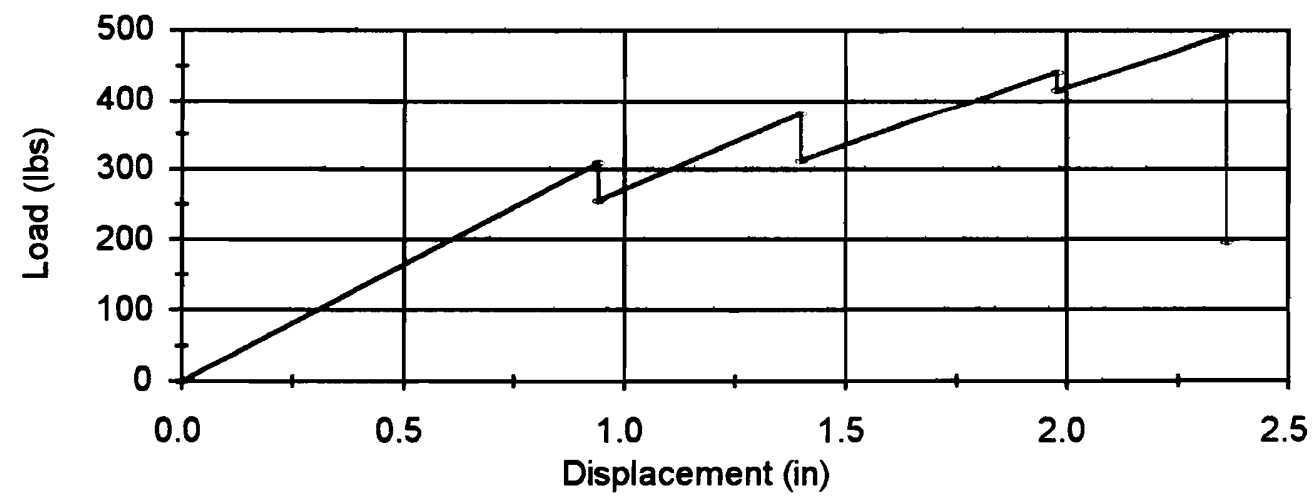

Figure 7.24 Frame response optimized for $500 \mathrm{lb}$ crush response with 2 cross-sectional geometries and one stacking sequence, with improved penalty.

For both of these cases the failure events were separated to some extent. In the symmetric 
frames, failures were occurring at $\pm \theta$ locations simultaneously. In these unsymmetrical frames, failure moves from one side of center at $\theta$ approximately $=5^{\circ}$, to the $\theta=90^{\circ}$ position, then to the other side of center at $\theta=-5^{\circ}$, then back to $\theta=90^{\circ}$ then to $\theta=-90^{\circ}$. and so on. However in the transition of failure from one location to another, some failures are still occurring spatially separated. Even so, the energy absorption of these designs should be less sensitive to random defects.

Additional optimizations were done using four independent cross section geometries, two on each side, and one laminate stacking sequence. .

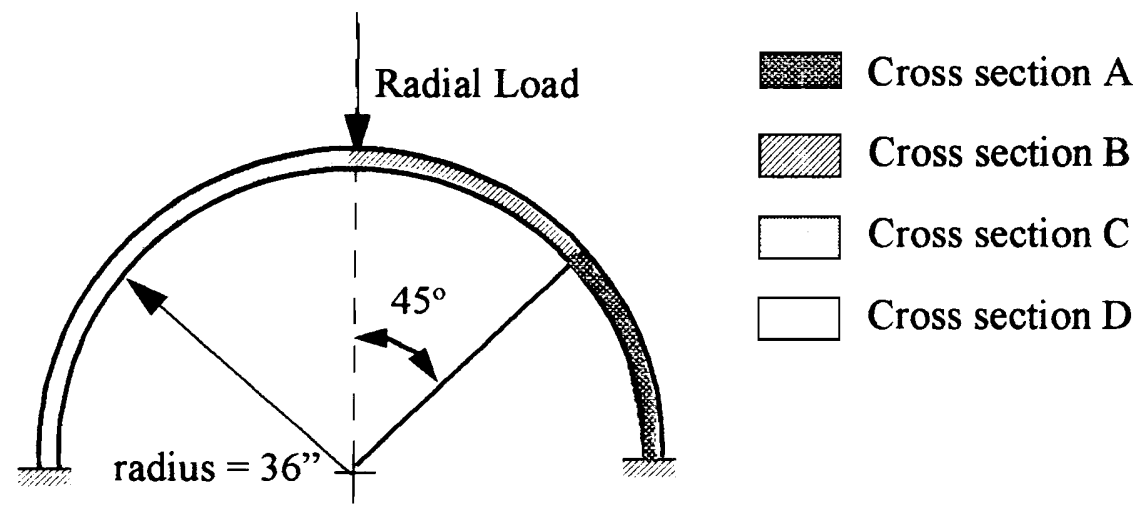

Figure 7.25 Division of cross section into two shape regions.

An additional increase of $10.12 \%$ in energy absorption was obtained for this case. The optimal design obtained for this analysis is described in Table 7.16, Table 7.17, Table 7.18, and Figure 7.26..

Table $7.16500 \mathrm{lb}$ case with 4 cross-sectional geometries

\begin{tabular}{cccc}
\hline \hline $\begin{array}{c}\text { Energy Absorbed } \\
\text { (in*lbs) }\end{array}$ & $\begin{array}{c}\text { Optimal Stacking } \\
\text { Sequence }\end{array}$ & $\begin{array}{c}\text { \% increase } \\
\text { in } \mathrm{E}_{\mathrm{a}}\end{array}$ & $\begin{array}{c}\text { \% increase in } \mathrm{E}_{\mathrm{a}} \\
\text { from baseline }\end{array}$ \\
\hline 745.7 & {$[-45 / 45 / 45 /-45]_{\mathrm{s}}$} & 17.77 & 660.91 \\
\hline \hline
\end{tabular}

a. \%increase from $500 \mathrm{lb}$ case with one laminate, and shape optimization (Table 7.9) 
Table 7.17 Optimal cross section shapes

\begin{tabular}{cccccc}
\hline \hline \multicolumn{3}{c}{ Section A } & \multicolumn{3}{c}{ Section B } \\
$\mathrm{Y}(3)$ & $\mathrm{Y}(6)$ & $\mathrm{Z}(3)$ & $\mathrm{Y}(3)$ & $\mathrm{Y}(6)$ & $\mathrm{Z}(3)$ \\
\hline 0.525 & 1.525 & 1.156 & 0.825 & 1.525 & 1.156 \\
\hline \hline
\end{tabular}

Table 7.18 Optimal cross section shapes

\begin{tabular}{cccccc}
\hline \hline & Section C & \multicolumn{3}{c}{ Section D } \\
$\mathrm{Y}(3)$ & $\mathrm{Y}(6)$ & $\mathrm{Z}(3)$ & $\mathrm{Y}(3)$ & $\mathrm{Y}(6)$ & $\mathrm{Z}(3)$ \\
\hline 0.75 & 1.600 & 1.156 & 0.600 & 1.525 & 1.156 \\
\hline \hline
\end{tabular}

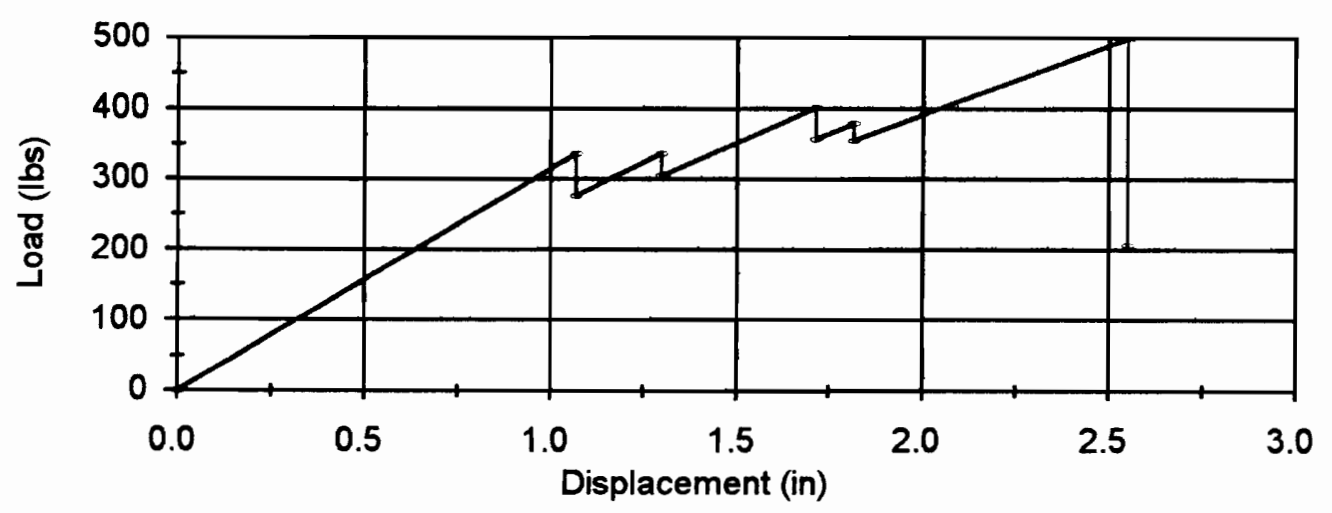

Figure 7.26 Frame response optimized for $500 \mathrm{lb}$ crush response with 4 cross sections and one stacking sequence. 


\section{CHAPTER 8}

\section{SUMMARY AND CONCLUDING REMARKS}

\subsection{SUMMARY}

The objective of this work was to develop a practical methodology for optimizing thinwalled composite fuselage frames for crashworthiness. The development process involved bringing together contributions from the five areas of crashworthiness, composite beam theory, finite element development, progressive failure analysis, and optimization. The progressive failure analysis methodology developed is specific to composite frames and preliminary comparisons to experimental data show a reasonable correlation given the level of simplification involved. The examples presented in Chapter 7 demonstrate the capability of the analysis and optimal design methodology.

A composite, curved, thin-walled beam theory including cross-sectional warping deformation was developed and presented in Chapter 2 . This theory combines various elements from previous research, along with several new elements, to form a unique composite thin-walled curved beam theory. The torsional warping theory due to Vlasov ${ }^{[5]}$, was extended to account for anisotropic material behavior for straight beams by Bauld and Tzeng ${ }^{[50]}$. Collins and Johnson ${ }^{[49]}$ added curvature in an early version of the present composite curved beam theory. The theory by Collins et. al ${ }^{[49]}$, was limited to cross sections symmetric about the plane of the ring or frame, and the section centroid was assumed to be 
the origin of the cross-sectional coordinates. The present work removes this symmetry requirement by assuming an arbitrary reference point in the cross section for the loads and displacements. This was important for the present work, since progressive failures can destroy cross-sectional symmetry. In a finite element analysis based on the present theory, the locus of reference points for each cross section forms a curve in space, and each cross section can be located freely with respect to that curve. This adds considerable flexibility and allows for more accurate modeling of structures. The present beam theory was also extended by incorporating the method of Sun and $\mathrm{Wu}^{[52]}$ to set either the reactive strains or reactive stress resultants to zero in the material law by the use of a simple parameter. Assuming the reactive resultants are zero when reducing the constitutive equations was found to result in a more accurate load response.

In Chapter 3, a methodology for calculating the modulus-weighted cross-sectional properties of composite, thin-walled, open cross section curved beams was developed. A method was devised which reduces the problem of section property calculation to an evaluation of geometric line integrals over each branch of the cross section, and a subsequent combination of these integrals using a modulus weighted summation over the contour. This methodology was implemented in a computer program that calculates all properties with respect to an arbitrary reference point, including the torsional warping properties. Automating the calculation of modulus weighted section properties was necessary for a practical progressive failure analysis since section properties change as a result of branch failures. This computer program fully automates the task of section property calculation, and has already found use in other applications.

The beam theory of Chapter 2 was implemented using a new finite element developed and tested as part of the present work. This finite element made the current approach to progressive failure analysis and optimization for crashworthiness possible. The finite element analysis is computationally fast and efficient, which makes optimization of crashworthiness practical. A key feature of having a special purpose analysis program as opposed to 
using a commercial program is increased speed. The advantage of having an independent analysis lies not only in the analysis for the response, but in access to the data generated by the response analysis. Profiling the current progressive failure analysis program revealed that approximately $70 \%$ or more of the computing time was spent in the post processing of material strains and calculation of failure. This is because each lamina in each section branch must be checked four times along the width, and six times along the length for failure. The process of transferring this large quantity of data between two separate computer programs (one for failure analysis, and one for structural adaptation) would appear to be an inefficient approach. The current approach combines the finite element curved beam analysis, and progressive structural modification process, in one analysis program. Development of the curved beam theory and the finite element made this combination possible.

The analysis verification studies performed in Chapter 5 demonstrate some limitations of composite curved beam theories in general. Comparisons to branched shell models confirm that cross-section distortion is probably the major source of inaccuracy in rigid crosssection curved beam theory. Because of the focus of the present work, this problem of cross-sectional distortion could not be examined in detail, but is considered interesting. Another interesting aspect that was revealed in these numerical tests was the importance of using the curved form of the contour warping function $\bar{\omega}$ (i.e. Eq.(2.44)) when calculating curved beam section properties. It is tempting and seems reasonable for thin frames to approximate $\bar{\omega}$ using the straight beam form Eq.(2.45). In the process of conducting the numerical experiments of chapter 5 it was discovered that approximation of $\bar{\omega}$ with the straight beam form of the equation results in a dependence of the torsional response on the reference point location, which is incorrect.

In Chapter 6, the progressive failure analysis is developed and presented. This methodology is modeled after Tsai's selective and progressive ply-by-ply degradation model for laminates, and is based on the principle of using efficient analysis models with predefined 
failure modes. Progressive material failure was defined as the principle mechanism of beam failure in the present work. Comparisons to the limited amount of test data available are encouraging. The trends in predicted energy absorption and failure response are considered good enough for preliminary design and optimization. Further comparisons to test data are required for a better understanding of this analysis methods limitations.

The progressive failure analysis was combined with a genetic algorithm in Chapter 7. This combination forms an optimal design methodology for increasing the crashworthiness of composite fuselage frames. Laminate stacking sequence for each section branch, and cross-sectional dimensions of each beam element are available as design variables. The genetic algorithm identifies those designs which have the highest energy absorption and do not violate the maximum failure load constraint. The optimal design methodology was numerically demonstrated to identify frame designs with substantially increased energy absorption capabilities.

Even with the analytical simplifications and assumptions that were made, the computational requirements of optimization for crashworthiness are large. An approximation of the solution time required for this optimization method using branched shell finite elements is compared to the actual requirements for the beam model in Table 8.1.

Table 8.1 Comparison of required solution time

\begin{tabular}{|c|c|c|c|c|c|}
\hline \multirow[b]{2}{*}{ Model } & \multicolumn{5}{|c|}{ Solution Time ( IBM RISC 6000) } \\
\hline & $\begin{array}{c}\text { Element } \\
\text { Assembly \& } \\
\text { Static Finite } \\
\text { Element } \\
\text { Solution }\end{array}$ & $\begin{array}{c}\text { Failure } \\
\text { Analysis \& } \\
\text { Modification } \\
\text { cycle }\end{array}$ & $\begin{array}{c}\text { Completion } \\
\text { of one } \\
\text { Progressive } \\
\text { Failure } \\
\text { analysis } \\
\text { (20+ analysis) }\end{array}$ & $\begin{array}{c}\text { One } \\
\text { Generation of } \\
10 \text { Individuals }\end{array}$ & $\begin{array}{c}\text { One } \\
\text { Optimization } \\
\text { of } 100 \\
\text { Generations }\end{array}$ \\
\hline $\begin{array}{l}\text { Present Compos- } \\
\text { ite Beam }\end{array}$ & $0.2 \mathrm{~s}$ & $0.4 \mathrm{~s}$ & $12.0 \mathrm{~s}$ & $2.0 \mathrm{~min}$ & $3 \mathrm{hrs}, 33 \mathrm{~min}$ \\
\hline $\begin{array}{l}\text { EAL Branched } \\
\text { Shell }\end{array}$ & $1 \mathrm{~min}, 33 \mathrm{~s}$ & $8.0 \mathrm{~s}$ & $33 \mathrm{~min}, 40 \mathrm{~s}$ & $5 \mathrm{hrs}, 37 \mathrm{~min}$ & $\begin{array}{c}23 \text { days, } 8 \mathrm{hrs}, \\
52 \mathrm{~min}\end{array}$ \\
\hline
\end{tabular}


The conventional approach to this class of progressive failure analysis problems is to use a materially and geometrically nonlinear analysis. It is estimated that adopting this approach would increase the computational requirements for the beam model by at least an order of magnitude. Judging from the solution times presented in Table 8.1, this additional increase in computational requirements might push optimization for crashworthiness to the limits of practicality. In retrospect, it appears that the choice to use a linear analysis was appropriate. Successful optimization often requires approximate analysis. The goal of producing a practical methodology for designing composite frames for crashworthiness was achieved.

\section{2 CONCLUDING REMARKS}

Significant conclusions from the present work are as follows:

- Optimal crashworthiness is obtained by maximizing the area under the force displacement response subject to an upper bound on failure load.

- Developing a practical methodology for optimization of crashworthiness requires a focused effort on increasing computational speed. This is accomplished by:

- Using fast and efficient analysis techniques.

- Making appropriate analytical simplifications and approximations when possible.

- Thin-walled composite curved beam theory increases analysis speed by over two orders of magnitude when compared to a branched shell analysis.

- Thin-walled composite curved beam theory is in general accurate, but is susceptible to errors when cross-sectional distortion is significant.

- The magnitude of error caused by cross section distortion is predominantly a function of beam curvature, and cross-sectional geometry. 
- Laminate stacking sequence, and loading act as secondary factors influencing errors due to cross-sectional distortion.

- A reasonably accurate and efficient progressive failure analysis of composite frames can be accomplished using a step-wise linear static analysis.

- This analysis methodology is limited to composite frames that fail in a characteristic brittle manner.

- This progressive failure analysis methodology is very fast and efficient when compared to alternative methods, which makes optimization for crashworthiness more practical.

- Comparison to available experimental data shows agreement acceptable for preliminary design and optimization.

- Optimization for maximum crashworthiness is feasible and practical using the current methodology.

- The current optimization methodology is relatively fast and produces optimal designs within a reasonable time on workstation type computer.

- For all design cases considered, the optimization methodology consistently identified frame designs which have a substantially increased capacity for energy absorption, and which do not violate the upper limit on failure load.

- The substantial increases in crashworthiness were obtained using relatively few independent design variables.

\subsection{FUTURE WORK}

Recommendations for future work include further testing and evaluation of the progressive failure analysis. Additional comparisons to experimental data are necessary to verify 
the results of Chapter 6, and identify deficiencies in the methodology. Additional failure modes such as crippling and bucking could be added to the progressive failure analysis and a frame specifically optimized for crashworthiness should be built and tested. Improvements could also be made to the genetic algorithm, by modifying the fitness and penalty functions to improve efficiency 


\section{REFERENCES}

[1] Huculak, P., "A Review of Research and Development in Crashworthiness of General Aviation Aircrafts: Seats Restraints and Floor Structures", Aeronautical Note NAE-AN-64. NRC No. 31334, Ottawa, National Research Council of Canada, Feb. 1990.

[2] Poon, C., "A Review of Crashworthiness of Composite Aircraft Structures.", Aeronautical Note NAE-AN-63, NRC No. 31276, Ottawa, February, 1990.

[ 3 ] Farley, G.L. Boitnott, R.L. and Carden H.D. "Helicopter Crashworthiness Research Program" NASA CP-2495, 1987.

[4 ] Carden, H.D. "Impact Dynamics Research on Composite Transport Structures", NASA TM-83691. March 1985

[ 5 ] Hayduk, R.J., Thomson, R.G., and Carden H.D., "NASA/FAA General Aviation Crash Dynamics Program - an Update", Forum, Vol 12, No. 3, 1979, pp. 147-156.

[ 6 ] Thomson, R.G., and Goetz, R.C., "NASA/FAA General Aviation Crash Dynamics Program - a Status Report", Journal of Aircraft, Vol 17, no.8, August 1980, p 584.

[ 7 ] Military Standard MIL-STD-1290A (AV), "Light Fixed and Rotary-Wing Aircraft Crash Resistance", Department of Defense, Washington, D.C., 26 September 1988

[ 8 ] Desjardins, S.P., Lannanen, D. H. and Singley, D. T., III, "Aircraft Crash Survival Design Guide", USARTL-TR-79-22A to 22E, Vol. I to V, 1980.

[ 9] Kindervater, Ch., Georgi, H., Korber, U., "Crashworthy Design of Aircraft Subfloor Structural Components", Energy Absorption of Aircraft Structures as an Aspect of Crashworthiness, AGARD Conference Proceedings No. 443, Luxembourg, May 1988, pp. 12.1 - 12.24.

[ 10 ] Hansen, J.S., and Tennyson, R.C., "The Dynamic Behavior of Stiffened Composite Fuselage Shell Structures", Energy Absorption of Aircraft Structures as an Aspect of Crashworthiness, AGARD Conference Proceedings No. 443, Luxembourg, May 1988, pp. 15.1-15.12. 
[ 11 ] Carper. C.H., and Burrows, L.T., "Evolving Crashworthiness Design Criteria", Energy Absorption of Aircraft Structures as an Aspect of Crashworthiness, AGARD Conference Proceedings No. 443, Luxembourg, May 1988, pp. $3.1-3.8$.

[12] Federal Motor Vehicle Safety Standards, Motor Vehicle Standard No. 208, Occupant Crash Protection, Superintendent of Documents, U.S. Government Printing Office. Washington, D.C., September 1981.

[ 13 ] Comments on Proposal for Enhanced Crash Tolerance of Small, FAR Part 23 General Aviation Aircraft with Fewer Than 10 Passenger Seats, Simula Inc., Technical Data Presentation at the General Aviation Safety Panel June 1984 Meeting, FAA Docket No. 23494, Small Airplane Airworthiness Review Program, Federal Aviation Administration, Kansas City, Missouri.

[14 ] Wittlin. G., "Transport Aircraft Crash Dynamics", Lockheed-California Company, NASA CR165851, 1982

[ 15 ] Cominsky, A., "Transport Aircraft Accident Dynamics", McDonnell Douglas Corp., NASA CR165850,1982

[ 16] Widmayer, E., and Brende, O.B., "Commercial Jet Transport Crashworthiness", NASA CR-165849, March 1989.

[17] Wittlin, G., "Aircraft crash dynamics:modelling verification and application", Structural Crashworthiness, Butterworths, London, 1983

[ 18 ] Carden. H. D., Boitnott, R. L., and Fasanella, E. L., "Behavior of Composite/Metal Aircraft Structural Elements and Components Under Crash Type Loads--What Are They Telling Us?", presented at the 17th Congress of the International Council of Aeronautical Sciences, Stockholm, Sweden, September 1990

[19] Farley, G.L., "Energy Absorption of Composite Materials", Journal of Composite Materials, Vol. 17, May 1983, pp. 267-279.

[ 20 ] Moas, E. Jr.. Boitnott, R. L.and Griffin, O. H. Jr. "An Experimental and Analytical Investigation on the Response of Gr/Ep Composite I-Frames",American Helicopter Society National Technical Specialists' Meeting on Rotocraft Structures: "Technology for the 1990's and Beyond", Williamsburg, Va. October 1991.

[ 21 ] Fleming, D.C., and Vizzini. A.J., "The Effect of Side Loads on the Energy Absorption of Composite Structures", Joumal of Composite Materials, Vol. 26, No. 4, 1992, pp. 486-499.

[22] Schmueser, D.W., Wickliffe,L.E., and Mase, G.T., "Front Impact Evaluation of Primary Structural Components of a Composite Space Frame", SAE Paper 880890, April 1988.

[23 ] Boitnott, R.L., Fasanella, E.L., Carden.H.D., and Calton, L.E., "Impact Response of Composite Fuselage Frames.", General Aviation Aircraft Crash Dynamics, SAE Publication SP-716, 1987. Also available as SAE Paper 871009, April 1987.

[24] Noor, A. K., and Carden, H. D., Computational Methods for Crashworthiness, Proceedings of a 
workshop held at Langley Research Center. Hampton, Virginia, September 1992.

[ 25 ] Hallquist, J.O., and Benson, D. J., "DYNA3D, a computer code for crashworthiness engineering." Report UCRL-95152. University of California, Lawrence Livermore National Laboratory, Livermore, CA. September 1986.

[ 26 ] Pifko, A. B., Winter, R. and Ogilvie, P. L., "DYCAST -- A Finite Element Program for the Crash Analysis of Structures," NASA CR 4040, Washington, D.C., January 1987.

[27] Gamon, M.A. Wittlin, G. and LaBarge, B., "KRASH 85 User's Guide -Input/Output Format(U)." Lockheed-California Company, Burbank, Ca. DOT/FAA/CT 85-10, July 1985.

[ 28 ] Majid, K.I.,Non-Linear Structures - MatrixMethods of Analysis and Design By Computers, Butterworths, London, 1972.

[29] Miles, J.C., "The Determination of Collapse Load and Energy Absorbing Properties of Thin Walled Beam Structures Using Matrix Methods of Analysis.", Int. Journal of Mechanical Science, Vol 18. 1976, pp. 399-405.

[ 30 ] Toi, Y., and Yang, H.J., "Finite Element Crush Analysis of Framed Structures", Computers \& Structures, Vol. 41, No. 1, 1991, pp.137-149.

[ 31 ] Murray, N. W., "The Static Approach to Collapse and Energy Dissipation in Some Thin-Walled Steel Structures", Structural Crashworthiness. Butterworths, London, 1983.

[ 32 ] Mahmood, H.F., and Paluszny,A., "Crash Analysis of Thin Walled Beam-Type Structures", SAE Paper 880894, April 1988.

[ 33 ] Bulokbasi, A.O., "Crash-Resistant Rotorcraft Preliminary Design Optimization",AHS forum, 1994

[ 34 ] Vander plaats, G. N., "CONMIN - A Fortran Program for Constrained Function Minimization," NASA Ames Research Center and U.S. Army Air Mobility R\&D Laboratory, Moffet Field, Ca, August 1973.

[ 35 ] Lust. R., "Structural Optimization with Crashworthiness Constraints.", Structural Optimization . Vol 4, 1992, pp. 85-89.

[ 36 ] Song, Ji Oh, "An Optimization Method for Crashworthiness Design", SAE Paper 860804, April 1986.

[ 37 ] DeVries, R.I., Saha, N.K.,Surulinarayanasami,P., and Zebrowski,M.P., "Structural Optimization of Beam Sections for Minimum Weight Subject to Inertial and Crush Strength Constraints.", SAE Paper 860805, April 1986.

[ 38 ] Vander Plaats, G. N., "User's Manual for ADS - A Fortran Program for Automated Design Synthesis Version 1.10", Engineering Design Optimization Inc., Santa Barbara, Ca. 1986.

[39] Tsai, S.W., Theory of Composites Design, Think Composites, Dayton, Ohio, 1992

[ 40 ] Petit, P. H., and Waddoups, M.E., "A Method for Predicting the Nonlinear Behaviour of Laminated 
Composites", J Composite Materials, Vol. 3, Jan. 1969, pp. 2-19.

[ 41 ] Tolson, S. and Zabaras, N., "Finite Element Analysis of Progressive Failure analysis in Laminated Composite Plates.", Computers \& Structures, Vol. 38. No. 3, 1991, pp.361-376.

[ 42 ] Pandey, A. K., and Reddy, J. N., "A Post First Ply Failure Analysis of Composite Laminates.", AIAA Paper No. 87-0898, 1987.

[ 43 ] Chang, F., and Chang, K., "A Progressive Damage Model for Laminated Composite Containing Stress Contrations.", J Composite Materials. Vol. 21, 1987, pp. 834-855.

[ 44 ] Bolukbasi, A. O., "An Analytical Method for Predicting the Energy Absorption Capability of Composite Stiffners.", McDonald Douglas Helicopter Company.

[ 45 ] Vlasov, V. Z., "Thin-Walled Elastic Beams", Second Edition, Moscow, 1959, Translated from Russian and published for the National Science Foundation, Washington, D.C., 1961.

[ 46 ] Gjelsvik. A., The Theory of Thin-Walled Bars, Wiley, New York, 1981.

[ 47 ] Murray, N.W., Introduction to the Theory of Thin-Walled Structures, Oxford, New York, 1984.

[ 48 ] Choi, I., and Horgan, C. O., "Saint-Venant's Principle and End Effects in Anisotropic Elasticity", Journal of Applied Mechanics, Vol. 44, No.3, Sept. 1977, pp. 424-430.

[ 49 ] Collins, J. S. and Johnson, E. R., "Static and Free-Vibrational Response of Semi-Circular GraphiteEpoxy Frames With Thin-Walled Open Sections" NASA Contractor Report 4287, March 1990.

[ 50 ] Bauld, N.R., Jr , and Tzeng, L, "A Vlasov theory for fiber-reinforced beams with thin-walled open cross-sections", Int. J. Solids Structures., Vol 20, No. 3, 1984, pp. 277-297.

[ 51 ] Sanders, J. L., Jr.,"An Improved First-Approximation Theory for Thin Shells", NASA Technical Report R-24, National Aeronautics and Space Administration, Washington DC, 1959

[ 52 ] Wu. X.X. and Sun. C.T., "Vibration Analysis of Laminated Composite Thin-Walled Beams Using Finite Elements", AIAA Journal, Vol 29, No. 5 , 1991 , pp. 736-742

[ 53 ] Coyette, J. P.,"An improved subroutine for the estimation of torsional properties of thin walled open cross-sections", Eng. Comput., Vol. 4, Sept. 1987, pp. 240-242.

[ 54 ] Roark, R. J., and Young, W.C., Formulas for Stress and Strain -Fifth Edition, McGraw-Hill, 1975

[ 55 ] Dawe, D.J., "Numerical Studies Using Circular Arch Finite Elements", Computers and Structures, Vol 4, 1974,pp 729-740

[ 56 ] Dawe, D.J., "Curved Finite Elements for the Analysis of Shallow and Deep Arches.", Computers \& Structures, Vol. 4, 1974, pp.559-580

[ 57 ] Engineering Analysis Language (EAL), Engineering Informations Systems, Inc.(EISI), San Jose, Ca.

[ 58 ] Mabson. G.E., "Effects of Curvature on Composite Material Beams", AIAA Journal, Vol. 28, No. 7 , pp. 1328-1331. 
[ 59 ] Peck, A.W., Design and Analysis of Curved Composite Components for Rotorcraft Fuselage Frames.. Ph.D. Dissertation, Rensselaer Polytechnic Institute, Troy, New York, August 1992.

[ 60 ] Rowlands, R.E., "Strength (Failure) Theories and their Experimental Correlation," in Handbook of Composites; vol 3;"Failure Mechanics of Composites," Sih,G.C., and Skurda, A.M. (eds.), Elsevier Science Publishers, Amsterdam, 1985.

[61] Puppo, A.H., and Evensen, H.A., "Strength of Anisotropic Materials Under Combined Stresses.", AlAA Journal, Vol 10, no.4, 1972, pp.468-474.

[62] Tsai, S.W., and Wu, E.M., "A General Theory of Strength for Anisotropic Materials.",Journal of Composite Materials, Vol 5, 1971, pp.58-80.

[63] Jones, R. M., Mechanics of Composite Materials, Scripta Book Co., Washington, D.C. 1975

[64] Think Composites, A division of ILT Corporation, Dayton, Ohio.

[65] van Wamelen, A.A., Optimal Design and Testing of Laminated Specimens to Evaluate Competing Composite Failure Criteria, Masters Thesis, Virginia Polytechnic Institute and State University, Blacksburg, Va. July 1993.

[ 66 ] Shin, Y.S., Haftka, R.T., Watson,L.T., and Plaut,R.T., "Design of Laminated Plates for Maximum Buckling Load", Journal of Composite Materials, Vol., 23, 1989, pp. 348-369

[67 ] Gurdal, Z., and Haftka, R.T., "Optimization of Composite Laminates", presented at the NATO Advanced Study Institute on Optimization of Large Structural Systems, Berchtesgaden, Germany, Sept. 23-Oct. 4, 1991.

[ 68 ] Holland, J.H.,Adaptation in Natural and Artificial Systems, University of Michigan Press, Ann Arbor, MI., 1975

[69] Goldberg, David E., Genetic Algorithms in Search, Optimization, and Machine Learning, AddisonWesley Publishing Co., Inc., 1989

[ 70 ] Hajela, P., "Stochastic Search in Structural Optimization: Genetic Algorithms and Simulated Annealing ",Structural Optimization: Status and Promise, Kamat,M.P. Editor,American Institute of Aeronautics and Astronautics Inc., Washington, D.C., 1993.

[ 71 ] Hajela, P., "Genetic Search - An Approach to the Nonconvex Optimization problem", AIAA Journal, Vol. 26, No. 7, 1990, pp. 1205-1210.

[ 72 ] Hajela, P., and Lin,C.Y., "Genetic Search Stratigies in Multicriteria Optimal Design",Structural Optimization, Vol. 4, 1992, pp. 99-107.

[ 73 ] Lin, C.Y., and Hajela,P., "Genetic Algorithms in Optimization Problems with Discrete and Integer Design Variables," Joumal of Engineering Optimization, Vol. 19, 1992, pp. 309-327.

[ 74 ] Le Riche, R. and Haftka, R.T., “Optimization of Laminate Stacking Sequence for Buckling Load Maximization by Genetic Algorithm", AIAA Journal, Vol 31, No. 5, May 1993, pp. 951-956 
[ 75 ] Le Riche, R., Composite Structures Optimization by Genetic Algorithms, Ph.D. Dissertation, Virginia Polytechnic Institute and State University, Blacksburg, Va. Oct. 1994.

[ 76 ] Richardson, J.T., Palmer, M.R., Liepens, G. and Hillard, M., "Some Guidelines for Genetic Algorithms with Penalty Functions", Proceedings of the Third Intermational Conference on Genetic Algorithms, George Mason Univ., Morgan Kaufmann Publishers, June 4-7, 1989, pp.191-197.

[ 77 ] De Jong, K.A., An Analysis of the Behoviour of a Class of Genetic Adaptive Systems, Ph.D. Dissertation, University of Michigan, 1975. 


\section{VITA}

Marshall Woodson was born in Roanoke Virginia, and grew up in nearby Botetourt County Virginia. He attended Lord Botetourt high school where he graduated as a member of the National Honer Society. High school interests included the debate team, and band. He earned a degree of distinction from the National Forensic League, and won the Virginia AA state championship in high school debate two years consecutively. After graduation from high school, Marshall entered the U.S. Navy and served as a hull maintenance technician with special training as a high pressure pipe welder. He entered Virginia Tech as an Engineering freshman in the fall of 1981 and graduated with a Bachelor of Science in Aerospace and Ocean Engineering in June of 1985. From June of 1985 through August of 1988, Marshall worked as a Stress and Fatigue Analysis Engineer for the Boeing Commercial Aircraft Company. He was a member of the 747/767 stress analysis group located in Everett Washington. Responsibilities included structural analysis of the aircraft fuselage, floor beams, and interior structure. From September of 1988 through August 1990, Marshall worked as a Stress and Fatigue Analysis Engineer for the Martin Marietta Astronautics group located in Denver Colorado. While at Martin Marietta he was assigned to a research and development branch of the space launch system division's Titan missile branch. In August of 1990, Marshall returned to Virginia Tech to begin work on a Ph.D. in Aerospace Engineering with a specialization in aerospace structures.

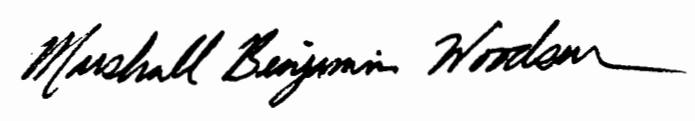

Marshall Benjamin Woodson 